\title{
SADDER BUT WISER
}

\author{
Zum Realismus der Selbsteinschätzung \\ hinsichtlich der Belastungswahrnehmung und der \\ motorischen Funktionswiederherstellung nach ZNS-Schädigungen \\ in Abhängigkeit vom Grad der Depressivität
}

Dissertation

zur Erlangung des sozialwissenschaftlichen Doktorgrades der Sozialwissenschaftlichen Fakultät der Universität Göttingen

$$
\begin{aligned}
& \text { vorgelegt } \\
& \text { von }
\end{aligned}
$$

Dipl.-Psych. Stefan Krause aus Frankfurt a. M.

Göttingen, 1997 
Berichterstatter:

Prof. Dr. A. Krüger

Mitberichterstatter:

Prof. Dr. A. Niklas

Tag der mündlichen Prüfung:

27.10.1997 
To feel good about ourselves we may have to judge ourselves more kindly than we are judged.

(Lewinsohn, Mischel, Chaplin \& Barton, 1980, S. 212) 


\section{Inhaltsverzeichnis:}

1 Einleitung $\quad 7$

2 Theoretischer und empirischer Hintergrund $\quad 11$

2.1 Ursachen und Folgen von ZNS-Schädigungen 11

2.1.1 Inzidenz und Ätiologie von ZNS-Schädigungen 12

2.1.2 Mechanismen der zerebralen Schädigung 14

2.1.3 Neurologische und neuropsychologische Implikationen $\quad 17$

2.1.3.1 Die Organisation der motorischen Systeme $\quad 17$

2.1.3.2 Die Fähigkeiten der motorischen Systeme $\quad 25$

2.1.3.2.1 Der Verlust der motorischen Fähigkeiten 36

2.1.3.2.2 Die Wiederherstellung der motorischen Fähigkeiten 39

2.1.3.2.2.1 Allgemeingültige Mechanismen der zentralnervösen Funktionswiederherstellung 39

2.1.3.2.2.2 Motorikspezifische Merkmale der zentralnervösen Funktionswiederherstellung 43

2.1.3.2.2.2.1 Physiotherapeutische Ansätze 43

2.1.3.2.2.2 Motorisches Lernen nach ZNS-Schädigungen 49

2.1.3.2.2.2.3 Training der konditionellen Fähigkeiten 53

2.2 Belastungswahrnehmung $\quad 56$

2.2.1 Leistung und Belastung $\quad 56$

2.2.2 Zum Begriff der Wahrnehmung $\quad 57$

2.2.2.1 Allgemeine Merkmale der Wahrnehmung $\quad 57$

2.2.2.2 Die Wahrnehmung der Belastung $\quad 59$

2.2.3 Belastungswahrnehmung als Steuergröße für Belastungsintensität 64

$\begin{array}{ll}2.3 \text { Depression } & 67\end{array}$

2.3.1 Grundlagen der Depression $\quad 67$

$\begin{array}{ll}\text { 2.3.1.1 Beschreibung der depressiven Störung } & 67\end{array}$

2.3.1.2 Epidemiologie $\quad 69$

2.3.1.3 Ätiologische Modelle $\quad 70$

2.3.1.3.1 Biologische Faktoren $\quad 70$

$\begin{array}{ll}\text { 2.3.1.3.2 Psychologische Faktoren } & 73\end{array}$

2.3.1.4 Exkurs I: Sport und Depression $\quad 81$

2.3.1.5 Exkurs II: Depression als Folge der ZNS-Schädigung 84

2.3.2 Der depressive Realismus 88 
2.3.2.1 Einführung in den depressiven Realismus $\quad 88$

2.3.2.2 Zum Begriff der Realität

2.3.2.2.1 Wissenschaftstheoretische Überlegungen zur Realität 90

2.3.2.2.2 Die Realität der Depressiven 92

2.3.2.3 Forschungsbereiche der Realismushypothese: Befunde und Implikationen 93

2.3.2.3.1 Einschätzung der Kontingenz 94

2.3.2.3.2 Erwartung und Vorhersage von Ereignissen 105

2.3.2.3.3 Attribution 112

2.3.2.3.4 Wahrnehmung und Erinnerung von Rückmeldungen 116

$\begin{array}{ll}\text { 2.3.2.3.5 Selbstbewertung } & 121\end{array}$

2.3.2.4 Diskussion des depressiven Realismus $\quad 124$

2.3.2.4.1 Erklärungsansätze des depressiven Realismus 127

2.3.2.4.2 Grenzen und einschränkende Faktoren des depressiven Realismus 128

2.4 Ableitung der sportpsychologischen Hypothesen 132

3 Methode 134

3.1 Versuchsplanung, Vorhersagen und statistische Hypothese $\quad 134$

3.2 Versuchspersonen $\quad 140$

3.3 Instruktion, Material, Hilfsmittel und Geräte 141

$\begin{array}{ll}\text { 3.3.1 Versuchsinstruktion } & 141\end{array}$

3.3.1.1 Handdynamometer 141

$\begin{array}{ll}3.3 .1 .2 \text { Tapping } & 141\end{array}$

3.3.1.3 Fahrradergometer 141

3.3.1.4 PC-Rehacom-Visuomotorik 142

3.3.1.5 Schraubenbrett 142

3.3.1.6 Erhebung der erwarteten Leistungsteigerung 142

3.3.1.7 Allgemeine Vorhersage des Wiederherstellungsgrads 142

3.3.2 Versuchsmaterial, Hilfsmittel und Geräte 143

3.3.2.1 Handdynamometer 143

3.3.2.2 PC-Programmodul „Tapping“ 143

3.3.2.3 Fahrradergometer 143

3.3.2.4 Herzfrequenzmesser 144

3.3.2.5 PC-Programm RehaCom-Visuomotorik 144

3.3.2.6 Schraubenbrett 145

3.3.2.7 Becks Depressions Inventar (BDI) 146

$\begin{array}{ll}\text { 3.3.2.8 Rating des Schweregrads der Behinderung } & 147\end{array}$ 
$\begin{array}{ll}3.4 \text { Versuchsdurchführung } & 147\end{array}$

4 Ergebnis $\quad 152$

4.1 Darstellung der H 1

4.2 Darstellung der $\mathrm{H} 2 \quad 158$

$\begin{array}{ll}\text { 4.3 Darstellung der H } 3 & 163\end{array}$

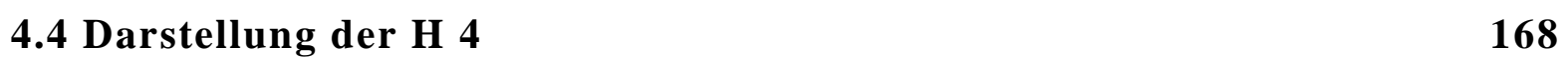

$\begin{array}{ll}\text { 4.5 Darstellung weiterer Ergebnisse } & 168\end{array}$

5 Diskussion $\quad 171$

6 Zusammenfassung $\quad 194$

7 Literaturverzeichnis $\quad 196$

8 Anhang $\quad 220$

8.1 Anhang A: Protokollblatt $\quad 220$

$\begin{array}{ll}\text { 8.2 Anhang B: BDI } & 221\end{array}$

8.3 Anhang C: Daten der Versuchspersonen 223

8.4 Anhang D: Werte der Versuchsbedingung 'Kraft' 223

8.5 Anhang E: Werte der Versuchsbedingung 'Schnelligkeit' 225

8.6 Anhang F: Werte der Versuchsbedingung 'Ausdauer' 226

8.7 Anhang G: Werte der Versuchsbedingung 'Koordination I' 226

8.8 Anhang H: Werte der Versuchsbedingung 'Koordination II' 228

8.9 Anhang I: Werte der Versuchsbedingung 'Belastungswahrnehmung' 228

8.10 Anhang J: Werte der unspezifischen Vorhersage 230

8.11 Anhang K: Ausmaß der Schädigung 231 


\section{Einleitung}

Begegnet ein Fußballtrainer einem Spieler, der dessen Siegessicherheit in bezug auf das anstehende Spiel nicht teilt, wird dieser alsbald als Pessimist abgetan. Das gleiche Bild bietet sich zeitgleich auch in der gegnerischen Mannschaft. Woher nehmen Sportler ihren scheinbar unbegrenzten Optimismus, der - da in jedem Wettkampf oder Sportspiel zumeist nur ein Gewinner existiert - nicht allein eine Folge zurückliegender Erfahrung sein kann? Sind Behauptungen wie „Ich werde gewinnen!“ nur Teil einer motivationssteigernden Selbstinstruktion (Baumann, 1993, S. 168 ff.) oder Teile des tatsächlichen Überzeugungssystems? Nehmen Sportler sich, ihre Erfolgswahrscheinlichkeit und Leistungsentwicklung positiver wahr, als es der Realität entspricht?

The position that psychologically disturbed individuals have more insight into reality than normal people has been voiced sporadically for centuries. Until recently, however, such arguments have not come from members of the scientific community. (Miller \& Moretti, 1988, S. 275)

Dieser Tatbestand hat sich - im Hinblick auf die depressive Störung - gewandelt. Nachdem die Assoziation einer negativ verzerrten Weltsicht mit dem Phänomen der Depression über Jahrzehnte die vorherrschende Meinung geprägt hatte, überraschten zwei Wissenschaftlerinnen Ende der siebziger Jahre mit gegenteiligen Beobachtungen. Diese wurden während einer Untersuchung im konzeptuellen Rahmen der Theorie der Gelernten Hilflosigkeit (vgl. Kap. 2.3.1.3.2, S. 73) gemacht, die die Genese der Depression durch die erfahrene und, daraus folgend, antizipierte stabile Zusammenhangslosigkeit von Verhalten und Umweltereignissen begründet sieht. Dabei sollte, gemäß Vorhersagen, die sich aus dieser Theorie ableiten, eine Unterschätzung des „,... degree of relationship between any two events“ (Alloy \& Abramson, 1979, S. 447) durch Depressive demonstriert werden. Statt dessen zeigte sich jedoch, was auch in der Folge oft repliziert werden konnte: Depressive schätzten die Höhe des Zusammenhangs vergleichsweise genau ein, während sie von Nichtdepressiven überschätzt wurde. Wie der Titel der Veröffentlichung dieser Wissenschaftlerinnen schon indiziert: Die Depressiven waren 'sadder but wiser' (Alloy \& Abramson, 1979). 
Nach etwa einem Jahrzehnt, in dem dieses Phänomen immer wieder Gegenstand der Untersuchung wurde, wendeten sich die Studien zunehmend von dem Versuch der einfachen Replikation ab; die Forschungsschwerpunkte verlagerten sich zu einem differenzierteren Vorgehen, wobei das Augenmerk auf die den depressiven Realismus beeinflussenden Faktoren gerichtet wurde. Auch wenn heute das anfängliche Interesse, ausgedrückt durch die Anzahl der Forschungsarbeiten auf diesem Gebiet, nachgelassen zu haben scheint - wie eine Stilblüte wirkt die kürzlich in der Wirtschaftswelt aufgestellte Hypothese „Managerial decision making will be hindered by positive affect“ (Staw \& Barsade, 1993, S. 308) - ist ein mehr substantielles Interesse an der mittlerweile zum festen Inventar des psychologischen Bestands gehörenden Realismushypothese ${ }^{1}$ geblieben.

Mit fortschreitender Theoriebildung in der Sportwissenschaft werden die untersuchten Gegenstandsbereiche zunehmend vielschichtiger, die untersuchten Bereiche scheinen von immer spezifischerer Bedeutung. Die vorliegende Arbeit eröffnet einen bislang unerforschten Teilbereich. Indem sie sich mit dem Gebiet der motorischen Leistungssteigerung und der Depression beschäftigt, werden Aussagen über die Zusammenhänge zwischen Depression und der Vorhersage von Leistungssteigerung möglich. Des weiteren - als gegenwartsbezogenes Element - stellt die durch die Belastungswahrnehmung gesteuerte Selbsteinschätzung einen zweiten Schwerpunkt dar. Durch diesen gelingt der Brückenschlag von der Realismushypothese zu der im Sport oft beobachteten Überbelastung, die als Folge einer Selbstüberschätzung - entstanden aus der Tendenz des bereits in vielen anderen Bereichen beobachteten 'illusory rosy glow' (Oliver, Raftery, Reeb \& Delaney, 1993, S. 408) - interpretiert werden kann².

Der vorliegenden Arbeit liegt die Vermutung zugrunde, daß Depressive der Realität auch hinsichtlich der Vorhersage von körperlichen Leistungssteigerungen und der Selbsteinschätzung, vermittelt durch die Belastungswahrnehmung, näher stehen, daß sie sich nicht unterschätzen, sondern im Vergleich zu Nichtdepressiven realistischer einschätzen. Die Untersuchung findet hierbei nicht im Bereich des Leistungs- oder Freizeitsports statt, sondern in einem Rahmen, in der die Steigerung der motorischen Fähigkeiten von viel essentiellerer Bedeutung ist: Bei Personen, die aufgrund von ZNS-Schädigungen basale

\footnotetext{
1 Die Ausdrücke 'depressiver Realismus', 'sadder but wiser', 'depressive realism hypothesis' und 'Realismushypothese' werden in der vorliegenden Arbeit synonym verwendet.

${ }^{2}$,When they are depressed, they lose the 'illusory rosy glow' and become more realistic, but perceive things less favorably, becoming 'sadder but wiser'“ (Oliver, Raftery, Reeb \& Delaney, 1993, S. 408).
} 
motorische Fähigkeiten verloren haben und diese, unterstützt durch therapeutische Maßnahmen, in einer neurologischen Rehabilitationseinrichtung wiederzuerlangen suchen.

In diesem Sinne folgt die vorliegende Untersuchung dem „Typ-bForschungsprogramm“ (Herrmann, 1976, S. 29). Bei diesem steht nicht ein Phänomenbereich, sondern eine bestimmte Theorie im Vordergrund. Innerhalb dieses Programms ist es Aufgabe der Untersuchung festzustellen, auf welche Phänomene und Phänomenbereiche diese Theorie anwendbar ist (Herrmann, 1976). Den theoretischen Hintergrund für die vorliegende Arbeit bildet der depressive Realismus, die beiden Phänomenbereiche teilen sich in die Vorhersage körperlicher Leistungssteigerung bei der Wiederherstellung nach ZNSSchädigung und in die Selbsteinschätzung, vermittelt durch die Belastungswahrnehmung, auf.

In einer Follow-up-Studie betrachteten Boné, Ladurner und Pichler (1988) das geschätzte Ausmaß der Funktionswiederherstellung nach Apoplexia cerebri ('stroke's) in bezug auf den Grad der Depressivität, wobei sie neben Eigen- auch Fremderhebungen durchführten: „Die Gegenüberstellung von Eigen- und Fremdbewertung zeigte, daß ... die depressive Gruppe ... sich signifikant $(\mathrm{p}<0,05)$ schlechter einstufte, wogegen Patienten mit rein neurologischer Symptomatik sich hinsichtlich des Rehabilitationserfolges besser beurteilten ( $p$ $<$ 0,01) als die Angehörigen“ (Boné, Ladurner \& Pichler, 1988, S. 61). Ungeklärt bleibt aber, ob dieses Phänomen auf die 'depressive distortion' oder den 'nondepressive illusory rosy glow' (s. o.) zurückzuführen ist. Folgt man der Hypothese der vorliegenden Untersuchung, wäre der 'nondepressive cognitive bias' hierfür verantwortlich.

Da die theoretischen Grundlagen dieser Arbeit zu einem großen Anteil dem anglo-amerikanischen Sprachraum entstammen, ist die Gefahr von sprachlichen Mißverständnissen durch eine uneinheitliche Übertragung zentraler Begriffe gegeben; aus diesem Grund folgt unmittelbar hinter dem bevorzugten deutschen Ausdruck der Originalbegriff ('original') in Klammern und einfachen Anführungszeichen. Querverweise innerhalb der Arbeit sind an ihrer Form zu erkennen; befindet sich in der Klammer nur die Seitenzahl, sind sie durch ein 'siehe' oder 'vgl.', je nach Inhalt, vor derselben zu erkennen. Bei gleichzeitiger Angabe des Kapitels handelt es sich immer um arbeitsinterne Verweise. Die Seitenzahlen hinter dem Kapitel beziehen sich entweder auf den Beginn des Kapitels oder direkt auf die Textstelle. Der Gebrauch der männlichen Form ('der Depressive') bezieht auch das

\footnotetext{
${ }^{3}$ auch: Schlaganfall, apoplektischer Insult, Apoplexie, Apoplexia cerebri
} 
weibliche Geschlecht mit ein.

Bevor der theoretische Hintergrund für diese Arbeit erläutert wird, sei der Blick noch für einen Moment nach Wien gerichtet. Dort war schon zur Zeit des ausklingenden zweiten Jahrzehnts dieses Jahrhunderts ein anderer, kaum wahrgenommener Zugang zur depressiven Realität geschaffen worden. Siegmund Freud (1917/1946) bemerkte im Rahmen einiger Gedanken zu Trauer und Melancholie:

Wenn er [der Depressive] sich in gesteigerter Selbstkritik als kleinlichen, egoistischen, unaufrichtigen, unselbständigen Menschen schildert, der nur immer bestrebt war, die Schwächen seines Wesens zu verbergen, so mag er sich unseres Wissens der Selbsterkenntnis ziemlich angenähert haben, und wir fragen uns nur, warum man erst krank werden muß, um solcher Wahrheit zugänglich zu sein. (Freud, 1917/1946, S. 432) 


\section{Theoretischer und empirischer Hitergrund}

Im folgenden werden die theoretischen und empirischen Hintergründe aufgezeigt, die den Rahmen der hierauf beschriebenen Untersuchung bilden. Einleitend werden Ursachen und Folgen von ZNS-Schädigungen geschildert. Im Anschluß daran wird die Organisation der motorischen Systeme sowie das Gebiet der Belastungswahrnehmung dargestellt, so daß, in Verbindung mit den anschließend detailliert aufgezeigten Problembereichen der Depression und des depressiven Realismus, die Ableitung der sportpsychologischen Hypothese hinreichend theoretisch gestützt ist.

\subsection{Ursachen und Folgen von ZNS-Schädigungen}

Ödipus verweist schon früh auf die Zeitlichkeit des Menschen. Haltung und Bewegung können als wesentliche Indikatoren der Funktion des Nervensystems angesehen werden. Schädigungen im Gehirn brechen in den normalen Ablauf des Nablassens von Funktionen ein.

Als Ödipus sich Theben näherte, stellte sich die Sphinx ihm in den Weg und gab ihm folgendes Rätsel auf: „Was geht am Morgen auf vier Beinen, zur Mittagszeit auf zweien und am Abend auf drei Beinen?“ Ödipus’ Antwort „,der Mensch“ war richtig, da wir als Kinder krabbeln, als Erwachsene aufrecht gehen und uns im Alter auf einen Stock stützen. Dieses Rätsel der Sphinx ist gleichzeitig auch eines, das die Natur des Menschen betrifft, und die Erwiderung von Ödipus zeigt nicht nur, daß er eine Antwort kannte, sondern auch, daß er die Bedeutung der Frage richtig einzuschätzen wußte. Das Rätsel der menschlichen Natur ist noch nicht gelöst. ... [man suche] eine mögliche Antwort dort, wo man sie der Logik nach am ehesten finden sollte: im Gehirn. (Kolb \& Wishaw, 1993, S. 1)

Und dort wird auch der 'sphinxsche Zyklus' durchbrochen: Schädigungen im Gehirn folgen ihren eigenen Gesetzen und brechen in den geordneten Kreislauf der Funktionsentwicklung bzw. des -verfalls ein. „Haltung und Bewegung können als wesentliche Indikatoren der Funktion des Nervensystems angesehen werden, sei es in Form unwillkürlicher Aktivität wie Atmung und Verdauung oder voluntarischer Aktivität wie Greifen, gestischer Bewegungen, Sprach- und Okulomotorik. .... Das Repertoire motorischer Fähigkeiten beim Menschen umfaßt elementare Funktionen wie z. B. die Entwicklung einer isometrischen Kraft weniger Muskeln beim Knopfdruck mit dem Zeigefinger bis hin zu komplexen Bewegungsabläufen beim Spielen eines Musikinstruments. Störungen solcher motorischer 
Funktionen können im Rahmen neurologischer Erkrankungen bei Schädigungen der an der Ausführung beteiligten zentralnervösen Strukturen beobachtet werden“ (Schnitzler \& Freund, 1995, S. 688). In den folgenden Kapiteln sollen die Rahmenbedingungen und neurologischen bzw. neuropsychologischen Implikationen von ZNS-Schädigungen und deren Rehabilitation genauer betrachtet werden.

\subsubsection{Inzidenz und Ätiologie von ZNS-Schädigungen}

Die jährliche Inzidenz der ZNS-Schädigungen liegt bei etwa 550.000, häufigste Ursachen sind der Schlaganfall und das Schädel-Hirn-Trauma. Die Ätiologie der zerebrovaskulären Störungen ist assoziiert mit Arteriosklerose, Arteriolosklerose, Embolie und Dissekate, der traumatischen ZNS-Schädigung mit Commotio cerebri, Contusio cerebri, epi- und subduralen sowie intrazerebralen Hämatomen.

„Unfälle verursachen jedes Jahr bei 300.000 Menschen Hirnschäden“, so die Feststellung des Kuratorium ZNS zu Beginn einer Literaturübersicht (Kuratorium ZNS, 1996, S. 2). Aufgeschlüsselt bedeutet dies eine Jahresinzidenz von „196 600 bis 200400 Patienten mit einer Hauptdiagnose aus dem SHT-Bereich und 101500 bis 104700 mit entsprechender Nebendiagnose“ (Kasten, Eder, Robra \& Sabel, 1997, S. 75). Die andere wichtige Gruppe der ZNS-Schädigung ist dem Formenkreis der zerebrovaskulären Störungen zuzuordnen, deren Häufigkeit aufgrund definitorischer Probleme nicht genau erfaßt werden kann, schätzungsweise aber bei etwa $5 \%$ der Bevölkerung liegt (Wittling, 1994). Anderen Quellen zufolge erleiden in der BRD etwa 200.000 Menschen pro Jahr einen Schlaganfall (Busse, Diener, Hennerici \& Sitzer, 1996, S. 2). Weitere Autoren nennen teils geringere, teils höhere Zahlen. Insgesamt scheint ein Bereich zwischen 100.000 und 300.000 Fällen von Hirngefäßerkrankungen als realistisch (für eine Übersicht siehe Kasten, Eder, Robra \& Sabel, 1997, S. 77). So kann für die BRD, „trotz gesunkener Inzidenzrate des Schlaganfalls“ (Vogel, 1994, S. 155), von einer Gesamtinzidenz für ZNS-Schädigungen pro Jahr von etwa 550.000 ausgegangen werden.

Die Ätiologie der traumatischen Hirnschädigungen geht auf äußere Gewalteinwirkungen zurück, wie sie bei Unfällen und Stürzen erfolgen. Zerebrovaskuläre Schädigungen hingegen haben viele Ursachen: Ungenügende Förderleistung des Herzens, Störung der Hämodynamik, Blutviskoseveränderung, intra- oder extrakranielle Strombahnhindernisse und Gefäßwandveränderungen, intrakranielle Drucksteigerungen sowie Gefäßwandblutungen und -zerreißungen (Wittling, 1994, S. 529). Die vier häufigsten dieser zerebrovaskulären Ursachen werden im folgenden detaillierter beschrieben (nach Liebsch, 1996, S. 33; Weiller, 1996, S. 47 ff.): 
Arteriosklerose: Arteriosklerotische Plaques der hirnversorgenden Halsgefäße und der großen Hirnarterie können Stenosen hervorrufen. Hinzu kommt teilweise noch ein thrombotischer Verschluß des Restlumens. Durch Blutdruckabfall kann bei einer vorbestehenden Stenose ein hämodynamischer Infarkt ausgelöst werden.

Arteriolosklerose: Durch Hyalinose und mikroatheromatöse Veränderungen werden bei einer chronischen arteriellen Hypertonie und/oder einem Diabetes mellitus Mikroinfarkte überwiegend der Aa. lenticulostriatae und der Aa. thalamoperforantes hervorgerufen.

Embolien: Sie entstehen bei der arterioarteriellen Embolie durch Verschleppung thrombotischen Materials aus ulzerierenden Plaques, bei Herzrhythmusstörungen durch Herzvitien, Endokarditis, Infarktnarben, Aneurysmen oder Kardiomyopathien, bei Beinvenenthrombosen durch Verschleppung über ein offenes Foramen ovale ins Gehirn, bei Fettembolie durch die Verschleppung von Knochenmark aus Trümmerbrüchen oder bei der Luftembolie durch Eindringen von Gasen (Luft) in den großen oder kleinen (Lungen-) Kreislauf.

Dissekate: Intimaeinrisse mit dissezierendem Aneurysma der A. carotis interna oder der A. vertebralis, die spontan oder posttraumatisch (mit einer Latenz von bis zu 20 Tagen) auftreten können.

Die Hauptfolgen des Schädel-Hirn-Traumas sind Commotio cerebri, Contusio cerebri, epi- und subdurale Hämatome sowie intrazerebrale Hämatome (Liebsch, 1996, S. 117 ff.), von denen neben der Contusio cerebri mit Substanzschädigung des Gehirns vor allem die Hämatome mit Raumforderung und umgebenden Hirnödemen im Rahmen des motorischen Funktionsverlusts von Bedeutung sind.

Die Contusio cerebri entsteht häufig im Rahmen eines Polytraumas. Das Gehirn stößt an der Stelle des Aufpralls am Knochen an ('coup'), durch den Unterdruck und das 'Zurückspringen' entstehen an der gegenüberliegenden Seite zusätzliche Schädigungen ('contre-coup'). Neben Rindenprellungsherden, die bevorzugt im Bereich der Frontal- und Temporalpole auftreten, entstehen auch periventrikuläre Läsionen und Einblutungen in das Marklager, in den Balken und in die Stammganglien. Das epidurale Hämatom liegt zwischen dem Schädelknochen und der Dura mater und entstammt zumeist einer Blutung der A. meningea media. Mit diesem Hämatom ist die kontralaterale Hemiparese assoziiert (vgl. S. 35). Das subdurale Hämatom weist ähnliche Symptome auf wie das epidurale, es tritt jedoch auch ohne eruierbare Traumen auf und breitet sich zwischen Gehirn und Kalotte aus. Das intrazerebrale Hämatom wird durch eine Blutung des Hirnparenchyms verursacht. 
Über $90 \%$ dieser Hämatome befinden sich, häufig oberflächennah lokalisiert, im Frontalbzw. Temporallappen (Liebsch, 1996, S. 119 ff.).

\subsubsection{Mechanismen der zerebralen Schädigung}

Schock, Ödem, Gehirndurchblutung, Transmitterfreisetzung, Autoneurotoxizität und Diaschisis stehen in Verbindung mit der Schädigung.

Unmittelbar im Bereich der Schädigung, ob durch eine Stichwunde oder Gewebeabtragung verursacht, entsteht ein Hohlraum, der sich im Verlauf der ersten Woche noch vergrößert, dann zunehmend schrumpft und nach einiger Zeit unter Umständen auch computertomographisch nicht mehr darzustellen ist (vgl. Kolb \& Wishaw, 1993, S. 452 ff.). Lediglich die in der Folge erweiterten Ventrikel sind dann sichtbar und können als Indiz für eine Schädigung gelten. In den ersten Stunden ist dieser Raum von einer Schicht toter Nervenzellen umgeben. In der nachfolgenden Schicht befinden sich sowohl tote als auch lebende Neuronen. Innerhalb von einem Tag infiltrieren Phagozyten dieses Gebiet, um abgestorbenes Gewebe aufzunehmen und abzubauen. Nach drei bis sieben Tagen wird dieses Gebiet neu kapillarisiert, der Raum ist nur noch von Gliazellen ausgefüllt. Das nekrotische Gewebe wird in einem Zeitraum, der sich über einige Monate erstrecken kann, vollständig entfernt. Betroffen sind jedoch nicht nur die im Fokus der umschriebenen Schädigung befindlichen Neuronen, auch die Axone weiter entfernt liegender Neuronen, die durch das geschädigte Gebiet ziehen, werden unmittelbar, die entsprechenden Neuronen somit mittelbar, betroffen. Die darauf folgende Degeneration der distal der Schädigung gelegenen Strukturen wird aufgrund der hohen Wahrscheinlichkeit, mit der dieser Vorgang stattfindet, häufig als das ‘einzige Gesetz der Neuroanatomie’ beschrieben (Kolb \& Wishaw, 1993, S. 453).

Die Schädigungsmechanismen der zerebralen Ischämie, die sich in einigen Punkten von der oben beschriebenen traumatischen Schädigung unterscheiden, fassen Leonhardt und Diener (1996) folgendermaßen zusammen:

Im Neuronenverband kommt es bei kritischer Minderperfusion aufgrund des Zusammenbruchs der Ionenpumpe zu einer präsynaptischen Glutamatfreisetzung. Die dadurch bewirkte Aktivierung von NMDA-, AMPA- und Metabotropenrezeptoren führt zu einem Einstrom von extrazellulärem $\mathrm{Ca}^{2+}$ und der Freisetzung von intrazellulärem $\mathrm{Ca}^{2+}$. Die unphysiologisch hohe Konzentration des universellen Second messengers $\mathrm{Ca}^{2+}$ aktiviert eine Vielzahl von Enzymsystemen. Phospholipasen induzieren eine Lipidperoxidation mit Entstehung freier Sauerstoffradikale. Proteasen führen zu einer globalen Einschränkung der Proteinsynthese. Endonukleasen beginnen DNA abzu- 
bauen. Die $\mathrm{Ca}^{2+} /$ calmodulinabhängige Stickoxidsynthetase wird induziert und produziert neurotoxisches Stickoxid. Durch die anaerobe Glykolyse kommt es zur Laktazidose mit Störung der Proteinsynthese und Anhäufung von freien Radikalen. Über Antiportmechanismen verläßt $\mathrm{K}^{+}$die Zelle und fördert weitere Depolarisationen mit Glutamatausschüttungen. Hier schließt sich der Circulus vitiosus der zerebralen Ischämie. (S. 88)

Weitere physiologische Ereignisse, die im Zusammenhang mit der Gehirnschädigung stehen, sind durch die Begriffe Schock, Ödem, Gehirndurchblutung, Transmitterfreisetzung, Autoneurotoxizität und Diaschisis umschrieben (nach Kolb \& Wishaw, 1993, S. 454 ff.):

Schock: Mashall Hall zeigte 1841, daß eine Schädigung des Rückenmarks zu einem zeitlich begrenzten Verlust der spinalen Reflexe führt, ein Umstand, der unter dem Begriff des 'spinalen Schocks' bekannt wurde. Er entsteht, indem dem Rückenmark Signale vom Gehirn vorenthalten werden, wobei die Dauer scheinbar von der Anzahl der Fasern abhängt, die das Rückenmark enthält. So ist bei einem Frosch beispielsweise kaum ein solcher Schock hervorzurufen, bei Katzen dauert er einige Stunden, beim Menschen kann er sogar einige Tage anhalten. Vermutet wird, daß ,... Rückenmarksneurone eine optimale Spontanladung aufweisen, die durch die Ausschüttung von Transmittersubstanzen aus deszendierenden Axonen verursacht wird. Unterbricht man diesen tonischen Einfluß, geht der Erregungszustand dieser Zellen zurück und kehrt erst allmählich wieder“ (a. a. O., S. 455). Dieses Prinzip ist auch auf andere Bereiche des ZNS zu übertragen; manche Zellgebiete scheinen im deafferentierten Zustand in ihrer Aktivität sehr beeinträchtigt zu sein, manche Zellen sterben in der Folge sogar ab.

Ödem: Eine Anschwellung des Gewebes (Ödem) als Folge eines Traumas kann durch den raumfordernden Prozeß Wirkungen auch auf entfernter gelegene Teilbereiche des Gehirns haben. Der ansteigende Druck kann die Funktion der Neuronen behindern, was beispielsweise in dem Zusammenhang von Ödembildung und IQ-Abfall seinen Ausdruck findet.

Gehirndurchblutung: Da das durch metabolische Aktivität des Nervengewebes freigesetzte $\mathrm{CO}_{2}$ die Durchblutungsrate kontrolliert, bewirkt die üblicherweise durch Gewebsverletzung herabgesetzte metabolische Aktivität einen geringeren Durchblutungsgrad des Gehirns; die Verringerung der Durchflußmengen kann sogar noch nach einem Monat nachgewiesen werden. Diese Minderdurchblutung betrifft auch die contralaterale Hemi- 
sphäre. Aufgrund der reduzierten Aktivierung durch die geschädigte Hemisphäre erfolgt ebenfalls in dieser eine Aktivitätsreduktion mit den einschlägigen Folgen.

Transmitterfreisetzung: In Tierversuchen wurde, in Analogie der Vorgänge bei einem Schlaganfall, die mittlere Hirnarterie durchtrennt. Der Catecholaminspiegel sank nach einigen Tagen, um nach drei Wochen wieder den ursprünglichen Wert und, im Sinne einer Überkompensation, teilweise sogar einen höheren Wert anzunehmen. Möglicherweise kann mit einer Catecholamin-Mangel-Hypothese das apathische Verhalten von Schlaganfallpatienten erklärt werden, gesicherte Erkenntnisse liegen aber, wie auch in Kapitel 2.3.1.3.1 (S. 70) beschrieben, auf diesem Gebiet noch nicht vor. Sicher ist nur, daß Hirnschädigungen in den komplexen interagierenden Wirkungsweisen der verschiedenen Neurotransmitter Veränderungen bewirken, die das Gleichgewicht zwischen diesen stören.

Autoneurotoxizität: Befunde, denen zufolge der Zelltod nicht immer sofort, sondern teilweise auch erst nach Stunden oder Tagen eintritt, legen nahe, daß nicht nur der Sauerstoffmangel für den Zelltod nach Schlaganfall oder Gehirnverletzung verantwortlich ist. Eine Ursache für diesen allmählichen Zelltod scheint darin begründet zu sein, daß durch Sauerstoffmangel der Neurotransmitter Glutamat ausgeschüttet wird. Dieser erregt die Nervenzellen in einem Maße, daß sie absterben.

Diaschisis: Nach Hummelsheim (1996 a) bezeichnet Diaschisis ,,... den Funktionsverlust bzw. die Funktionsänderung (z. B. durch den weggefallenen exzitatorischen oder inhibitorischen Input) in umschriebenen Regionen des Hirns, der sich in der Folge einer anderswo lokalisierten, jedoch anatomisch verbundenen Hirnläsion einstellt“ (S. 136). So kommt es beispielsweise bei einem Infarkt im Versorgungsgebiet der A. cerebri media in der kontralateralen Hemisphäre zur Diaschisis, deren Lokalisation und Ausprägung durchaus mit der primären Schädigung im Zusammenhang steht. 


\subsubsection{Neurologische und neuropsychologische Implikationen}

Verschiedene Zusammenhänge zwischen Schädigung, Funktionsverlust und -wiederherstellung werden dargestellt. Unteschiedliche Modelle konkurrieren miteinander oder ergäzen sich.

Die Verbindung zwischen der Schädigung und dem Funktionsverlust bzw. die psychologische Entsprechung im physiologischen Korrelat sind Inhalt der folgenden Kapitel. Es wird gezeigt, wie sich der Verlust der motorischen Fähigkeiten vor dem Hintergrund ihrer Funktionsweisen ereignet und welche Prozesse an der Wiederherstellung beteiligt sind.

\subsubsection{Die Organisation der motorischen Systeme}

Die einzelnen involvierten Subsysteme sind das Rückenmark, der Hirnstamm, das Kleinhirn, die Basalganglien, Teile des Thalamus und der Neocortex. Zwei Systeme agieren unabhängig voneinander: Der Hirnstamm und der Neocortex. Ein Blick auf die Phylogenese hilft, einige Phänomene verständlicher zu machen. Die Systeme werden unter Einbeziehung aktueller Forschungsergebnisse vorgestellt.

Nach der Übersicht über Prävalenz und Funktionsweise der ZNS-Schädigungen werden nun die zugrundeliegenden Systeme genauer betrachtet. Hierzu gehören die Organisation der motorischen Systeme, die Komponenten der motorischen Leistungsfähigkeit (Kap. 2.1.3.2, S. 24) und die detailliertere Darstellung, wie es zu dem Verlust der koordinativen und konditionellen Fähigkeiten kommen kann (Kap. 2.1.3.2.1, S. 35).

Um die Organisation der motorischen Systeme (nach Kolb \& Wishaw, 1993, S. 97 ff.; Poek, 1994, 86 ff.; Schnitzler \& Freund, 1995, S. 687 ff.; vgl. auch 'Der Verlust der motorischen Fähigkeiten', Kap. 2.1.3.2.1, S. 35 und 'Motorikspezifische Merkmale der zentralnervösen Wiederherstellung' Kap. 2.1.3.2.2.2, S. 43) verstehen zu können, ist ein erster Blick in die phylogenetische Entwicklung hilfreich. Die Abfolge scheint sich auf nachstehende Sequenzen zu fokussieren:

- Ganzkörperbewegungen

- Koordinierte Bewegungen der Gliedmaßen

- Unabhängige Bewegungen der Gliedmaßen

- Unabhängige Bewegungen der Finger

Unabhängige Bewegungen der Finger scheinen durch die stammesgeschichtlich jüngsten Zellen des Neocortex kontrolliert zu sein, die direkt zum Rückenmark projizieren und erst bei höheren Lebewesen anzutreffen sind. Ontogenetisch sind die gleichen Phasen in der Entwicklung eines Kindes zu beobachten, das sich nach der Geburt nur mit dem ganzen Körper bewegt, dann, nach der isolierten Bewegung der Arme und Beine in den darauffolgenden Monaten, zwischen dem achten und elften Monat den 'Pinzettgriff' beherrscht, der den selektiven Gebrauch des Daumens und des Zeigefingers voraussetzt. Anatomisch geht 
mit diesem Vorgang die Myelinisierung der entsprechenden Axone einher, die beispielsweise bei der Pyramidenbahn erst mit dem zweiten Lebensjahr abgeschlossen ist.

Die Anatomie der motorischen Systeme ist in seiner Komplexität anderen höheren ZNSFunktionen wie Wahrnehmung oder Kognition vergleichbar. Zu den involvierten Subsystemen gehören das Rückenmark, der Hirnstamm, das Kleinhirn, der Thalamus, die Basalganglien und der Neocortex (Kolb \& Wishaw, 1993). Zwei Systeme können unabhängig voneinander agieren: Der Hirnstamm und der Neocortex.

Der Hirnstamm, zu dem die Medulla oblongata, die Pons, das Mesencephalon, das Diencephalon und die subcortikalen Endhirnkerne gehören (Pschyrembel, 1994), ist komplexer aufgebaut als das Rückenmark, wenngleich dessen grundlegende Architektur noch erkennbar ist. Sensorische und motorische Fasern des Rückenmarks setzen sich in den sensorischen und motorischen Fasern der zwölf Hirnnerven fort, die auf Höhe der Pons entspringen (Faller, 1995, S. 399). Das ventromediale System des Rückenmarks, welches den vestibulospinalen, den reticulospinalen und den tectospinalen Trakt enthält, hat seinen Ursprung in den gleichnamigen Kernen des Hirnstamms (vestibuläre Nuclei, Retikulärformation, Tectum). Wichtig sind diese vor allem für die Initiierung von Ganzkörperbewegungen, in denen viele Muskelgruppen der axialen und proximalen Körpermuskulatur einbezogen sind. Die Fasern dieses Systems kreuzen nicht auf die contralaterale Seite; sie projizieren zu den medial gelegenen Interneuronen in der intermediären Zone des Rückenmarks. Aufgrund bestimmter Neurotransmitter, die im retikulären System vorhanden sind, wird vermutet, daß diese weniger für die Auslösung als vielmehr für die Modulation der Gesamtbewegung zuständig sind. Das laterale System, dessen Fasern zu der contralateralen Seite kreuzen, entspringt dem Nucleus ruber. Damit übt dieser vor allem die Kontrolle über die distale Muskulatur aus. Unabhängige Bewegungen von Händen und Füßen werden so erst möglich.

Die beiden anderen Gangverläufe im Rückenmark sind für die cortikale Reizweiterleitung vorgesehen. Es handelt sich hierbei um den lateralen und den ventralen corticospinalen Trakt. Beide bilden zusammen die Pyramidenbahn, deren Hauptaufgabe in der Initiierung der Willkürmotorik und der Hemmung der Motoneuronen besteht. Der Cortex hat viele Möglichkeiten, Bewegungen auszulösen, sie zu steuern oder Einfluß auf diese auszuüben (s. u.). Der direkte Weg führt über die bereits erwähnte Pyramidenbahn zu den corticospinalen Trakten, die mit den Moto- und Interneuronen des Rückenmarks verbunden sind und zu etwa $40 \%$ der Präzentralregion entstammen. Sie kreuzen auf Höhe der Pons auf die contralaterale Seite (80-90 \%), um im lateralen corticospinalen Trakt zu deszendieren, oder 
sie verlaufen ungekreuzt im ventromedialen Trakt (10-20\%). Andere Verbindungen, die corticobulbären Fasern, führen zu den Kernen des Hirnstamms und sind somit an der Kopfsteuerung beteiligt, während corticofugale Fasern nur zunächst zu Hirnstammkernen führen. Diese sind ihrerseits Ausgangspunkt für Faserzüge, die den Anteil der vom Hirnstamm ausgehenden lateralen und ventromedialen Systeme bilden. Somit kreuzen die einen Fasern, während die anderen auf der ipsilateralen Seite verbleiben. Insgesamt erreichen nur etwa $50 \%$ der pyramidalen Bahnen das Rückenmark, die übrigen sind mit den Systemen des Hirnstamms verschaltet.

Da in der Area 4 der Brodmannschen Cytographierung (Brodmann, 1909; s. u.) verschiedene topologische Punkte unterschiedliche Funktionen repräsentieren, führt eine Markierung der jeweiligen Abschnitte mit den dazugehörigen Funktionen zu dem in den Größendimensionen verzerrten Abbild eines Menschen, dem sogenannten 'Homunculus' (Kolb \& Wishaw, 1993, S. 69), der sich entlang der Horizontalachse vor dem Sulcus centralis ausstreckt. Die Verzerrung entsteht, da nicht die Größe des ausführenden Organs über die Anzahl der Neuronen entscheidet, sondern die Wichtigkeit bzw. das Ausmaß der Differenzierung, die innerhalb der Bewegung des Organs möglich ist. So ist der Daumen beispielsweise überdimensional groß, während der Rumpf einen vergleichsweise bescheidenen Raum einnimmt. Eine dem 'Homunculus' ähnliche Repräsentation der Effektoren ist unter anderem auch im Rückenmark, in der Area 6, dem somatosensorischen Cortex und innerhalb der Pyramidenbahn in der Capsula interna anzutreffen. Mit dem Rückenmark weist der präzentrale 'Homunculus' folgende direkte Verbindungen auf (nach Kolb \& Wishaw, 1993, S. 106):

- Zellen aus den motorischen Rindenbereichen der Finger und Zehen sind direkt mit den Motoneuronen in der dorsolateralen Zellgruppe der grauen Substanz des Rükkenmarks verbunden. Der Faserzug kreuzt auf die contralaterale Seite und ist Teil der Pyramidenbahn. Dieser Bestandteil der motorischen Organisation ist nur bei denjenigen Lebewesen vorhanden, die ihre Zehen unabhängig voneinander bewegen können. Neben den Primaten, zu denen an dieser Stelle auch der Mensch gezählt wird, sind es beispielsweise Katzen, Hamster oder Waschbären.

- Ebenfalls zur Pyramidenbahn gehören die Fasern derjenigen Zellen, die für die Kontrolle von Fingern, Hand und Gliedmaßen zuständig sind. Sie ziehen entlang der contralateralen Seite zu Interneuronen, die sich im dorsolateralen Teil der intermediä- 
ren Zone des Rückenmarks befinden. Ihre Aufgabe ist es scheinbar, relativ unabhängige Bewegungen von Arm und Hand zu ermöglichen.

- Rindenbereiche, in denen der Körper motorisch repräsentiert ist, projizieren zu Interneuronen im ventromedialen Teil der intermediären Zone des Rückenmarks, sowohl ipsi- als auch contralateral. Der eine Teil kreuzt mit der Pyramidenbahn, der andere bildet den ventralen corticospinalen Trakt. Hierbei liegen die Aufgaben vermutlich in der Steuerung der proximalen Anteile der Gliedmaßen, die für das Gehen und das Aufrichten aus einer geneigten Position von Bedeutung sind.

Verschiedene Cortexgebiete haben einen direkten oder indirekten Einfluß auf die Motorik, so daß „... für die Kontrolle motorischer Funktionen beim Menschen und Primaten ... die Integrität verschiedener motorischer Areale innerhalb der Großhirnrinde von entscheidender Bedeutung“ (Schnitzler \& Freund, 1995, S. 689) ist. Hierbei muß die Integration sowohl innerhalb der motorischen Areale erfolgen, als auch in bezug auf Systeme, die die Verarbeitung sensorischer oder kognitiver Prozesse zur Grundlage haben.

Die motorischen Areale liegen im Frontalhirn. Es handelt sich hierbei um den bereits angesprochenen motorischen, den prämotorischen und den supplementärmotorischen Cortex (MC, PMC und SMA), die nach Brodmann (1909) in den Arealen 4 (MC) und 6 (PMC und SMA) lokalisiert sind. Innerhalb der Area 6 nimmt der PMC die seitliche Fläche vor dem MC ein, während der SMA medial lokalisiert ist. Die Verarbeitung der Sensorik findet hauptsächlich im Parietallappen statt. Hierbei entsprechen die Felder 1 bis 3 dem primär somatosensorischen Cortex (SI). Hinter diesem liegen die primären und sekundären, unimodalen, somatosensorischen Assoziationsareale (SAI und SAII). Die Lokalisation der polymodalen Areale ist beim Menschen noch weitgehend ungeklärt, vermutet wird sie in dem Gebiet des Gyrus angularis und dem des Gyrus supramarginalis. Bei der Planung von Bewegung scheint wiederum der supplementärmotorische Cortex von herausragender Bedeutung zu sein, da bei der mentalen Ausführung von Bewegungen nur dieses Areal, nicht aber der primärmotorische Cortex aktiviert wird (Kolb \& Wishaw, 1993, S. 106). Durch diesen Prozeß erfolgt der Schritt von der Kognition zur Bewegung.

Wie bereits angesprochen, ist der motorische Cortex über direkte corticospinale Projektionen der Pyramidenbahn mit den zumeist kontralateralen Inter- und Motoneuronen verbunden, die die gemeinsame Endstrecke bilden und auf diese Weise die Integration spinaler Reflexe und zentralmotorischer Prozesse ermöglichen (s. o.; Schnitzler \& Freund, 1995, S. 689). Der MC seinerseits erhält direkte Projektionen von den prämotorischen Arealen und dem somatosensorischen Cortex. Da er auch indirekt von den subcortikalen Strukturen Ba- 
salganglien und Kleinhirn beeinflußt wird, scheint seine Hauptaufgabe in der Funktion als Schaltstelle zu liegen. Er ,... integriert diese Eingangssignale zu einem efferenten, über die Pyramidenbahn zu den spinalen Inter- und Motoneuronen projizierten Ausgangssignal, das die Verteilung, den Zeitgang und das Ausmaß muskulärer Aktivierung spezifiziert“ (Schnitzler \& Freund, 1995, S. 689). Die Aufgabe der Efferenzen, die vom motorischen Cortex zum prämotorischen, supplementärmotorischen und somatosensorischen Cortex, zum Thalamus, zu den Basalganglien und zum Kleinhirn führen, sind bislang ungeklärt. Es gilt aber als wahrscheinlich, daß diese Efferenzkopien die entsprechenden Strukturen über die intendierte Bewegung informieren und so deren Aktivität beeinflussen sollen.

Im prämotorischen Cortex erfolgt nach traditionellem Verständnis die höchste Stufe der motorischen Steuerung und Programmierung (Fulton, 1935). Mittlerweile gilt der PMC aber als dem MC sowohl über- als auch nebengeordnet. Über bilateral organisierte Bahnen projiziert er zur medialen pontomedullären Formatio reticularis, die dort unter Einbeziehung von tekto- und vestibulospinalen Fasern auf retikulospinale Projektionen umgeschaltet werden (Lawrence \& Kuypers, 1968). Von Bedeutung ist dieses System für die axiale Halte- und Stützmotorik. Im Gegensatz zur SMA sind bei der internen Bewegungsprogrammierung keine Potentiale ableitbar. Über direkte Verbindungen zu dem primär somatosensorischen Cortex verfügt dieser Teil des Cortex nicht, wohl jedoch über Verbindungen zum MC und PMC beider Hemisphären sowie zu den parietalen Arealen 7 und 19, wobei Areal 7 eine wichtige Funktion in Verbindung mit der visuellen Steuerung des Arms übernimmt, während Areal 19 Teil des visuellen Assoziationscortex ist. Wird bei einer Person der PMC gereizt, reagiert diese zumeist mit proximalen Bewegungen; bei den Armen kommt es zur Abduktion und Elevation, die häufig von Rumpf-, Kopf- und Blickwendungen begleitet werden. Komplexere erlernte Handlungen wie Sprechen oder Schreiben konnten jedoch bislang nicht evoziert werden (Penfield \& Rasmussen, 1950).

Die supplementärmotorische Area erhält afferente Eingänge aus den Basalganglien via Thalamus, aus den primär und sekundär somatosensorischen Gebieten und aus dem parietalen Assoziationscortex. Wie der motorische Cortex - und im Gegensatz zum prämotorischen Cortex - projiziert sie direkt über die Pyramidenbahn in spinale Strukturen. Bedeutsam scheint sie für die Planung, Vorbereitung und Initiierung von Bewegungen zu sein (Goldberg, 1985), wofür, neben den vor einer Bewegung ableitbaren Potentialen, sowohl die Beobachtungen eines akinetischen Mutismus nach bilateraler Läsion als auch die einer transienten Hemiakinese bei einseitiger Läsion sprechen (Laplane, Talairach, Meininger, Bancaud \& Bouchareine, 1977). Neuere Untersuchungen zeigen, daß diesem Cortexgebiet vor allem bei intern erinnerten Bewegungen eine wichtige Rolle zukommt; dies ist bei sen- 
sorisch geführten Bewegungen jedoch nicht der Fall (Deiber, Passingham, Colebatch, Friston, Nixon \& Frackowiack, 1991).

Die Ausarbeitung motorischer Programme, für die die Sinnesinformationen wichtig sind, ist Aufgabe der parietalen Anteile des Cortex. Läsionen in diesem Gebiet führen infolgedessen zu visuo-motorischen bzw. taktil-motorischen Fehlleistungen.

Angesichts der engen Verbindungen von Sensorik und Motorik stellt sich die Frage, ob Bewegungen ohne sensorische Rückmeldung überhaupt möglich sind. Schon auf der Ebene des Rückenmarks bilden sensorische Afferenzen synaptische Verbindungen zu Motoneuronen, was beispielsweise den Stellreflex oder die motorische Reaktion auf einen Schmerzreiz hin erklärt, bevor dieser cortikal wahrgenommen wird. Auf cortikaler Ebene weisen die Areale 2, 3 und 1, die den postzentralen Gyrus darstellen, direkte Projektionen zum motorischen Cortex im Gyrus präcentralis auf. Experimente mit Rhesusaffen, die in verschiedenen Versuchsanordnungen deafferentiert wurden, zeigten, daß es bei diesen motorische Programme im Zentralnervensystem geben muß, die unabhängig von sensorischen Reizen oder Rückmeldungen abrufbar sind (vgl. Kolb \& Wishaw, 1993, S. 111). Selbst wenn die Affen kurz nach der Geburt der Deafferentierung unterzogen und zusätzlich der visuellen Kontrolle beraubt wurden, konnten sie eine Reihe von Bewegungen ausführen, einschließlich des Pinzettgriffs. Auch bei Menschen, bei denen durch Krankheit bedingt afferente Signale fehlen, zeigen viele Bewegungen Einschränkungen in der Qualität der motorischen Ausführung, ohne daß diese Personen aber die grundsätzliche Fähigkeit zu diesen Bewegungen verloren haben.

Zwei weitere Systeme tragen neben Neocortex und Hirnstamm wesentlich zur Bewegung bei, wenn auch auf indirektem Weg (vgl. S. 18); es handelt sich hierbei um die Basalganglien und das Cerebellum. Die Funktionsweise der Basalganglien, zu denen im engeren Sinne Striatum (mit Nucleus caudatus und Putamen), Claustrum und Corpus amygdaloideum, im weiteren Sinne auch Pallidum, Nucleus subthalamicus, Nucleus ruber, Substantia nigra und Formatio reticularis gehören (Pschyrembel, 1994), ist nicht genau bekannt, was zum einen an ihrer komplexen Verschaltung liegt, zum anderen an dem Umstand, daß die Fasern des Neocortex so dicht an ihnen vorbeilaufen, daß die Unterscheidung, ob eine Störung auf einer Verletzung der Basalganglien oder der vorbeiziehenden Fasern beruht, häufig nur hypothetisch getroffen werden kann. Zwei Funktionen scheinen jedoch sicher: Einerseits sind die Basalganglien Teil eines Schaltkreises, der fast den gesamten Neocortex mit dem Motocortex verbindet. Andererseits modulieren sie die Information, was aus dem Umstand geschlossen werden kann, daß das Fehlen einiger Bestandteile zu akinetischen 
bzw. hyperkinetischen Phänomenen führt. Weitere Funktionen liegen vermutlich in der Steuerung langsamer und komplexer Bewegungen. Insbesondere der komplette Ausfall bei einer Hemiplegie (vgl. Kap. 2.1.3.2.1, S. 35) scheint nur durch den Ausfall der Anteile des Areals 4 und der Basalganglien erklärt werden zu können. Genauere Abgrenzungen sind jedoch methodisch schwierig und gelten bislang als noch ungeklärt.

Einen anderen Zugang wählten Müller \& Konczack (1995), die anhand einer Analyse der Parkinsonschen Krankheit, der am häufigsten auftretenden Krankheit der Basalganglien (Poek, 1994, S. 341), einige ihrer Aufgaben zu extrahieren versuchten: „Eine Möglichkeit, allgemeinen Prinzipien zur Bewegungssteuerung auf die Spur zu kommen, ist das Studium von Krankheiten, die bestimmte motorische Defizite aufweisen. Viele neurologische Erkrankungen zeigen charakteristische pathologische Bewegungsmuster, deren Analyse Rückschlüsse auf die Gesamtorganisation des motorischen Systems zulassen“ (Müller \& Konczack, 1995, S. 665). Das Hauptdefizit dieser Krankheit entsteht durch den Untergang einer durch den Neurotransmitter Dopamin charakterisierten Bahn vom subcortikalen Kerngebiet Substantia nigra zum Striatum (a. a. O., S. 666). Die Folge ist eine reduzierte Projektion des Thalamus zum prämotorischen Cortex und eine Verringerung der Innervation von Hirnstammzentren, die für die Haltemotorik zuständig sind. Akinese, Rigor und Tremor, die Leitsymptome dieser Krankheit, können als Konsequenz der Veränderung im Bereich der Basalganglien interpretiert werden, wobei besonders das Phänomen der Akinese das Forschungsinteresse geweckt hat. Hierbei ist nicht die Auswahl des motorischen Programms beeinträchtigt, da sich Patienten bei Wahlreaktionsaufgaben den Werten der Gesunden deutlich annäherten und auch beim Lernen neuer Aufgaben keine Schwierigkeiten hatten (Müller \& Konczack, 1995). Elektromyographische Ableitungen zeigten, daß zwar die richtigen Muskelagonisten aktiviert wurden, die initiale Muskelaktivierung aber nicht ausreichte, so daß mehrere Entladungen ('bursts') notwendig wurden (Hallett \& Khoshbin, 1980). Des weiteren finden sich längere Latenzen bis zum Kraftgipfel, niedrigere Kraftanstiegsraten und das Kraft-Zeit-Profil zeigt viele Unregelmäßigkeiten. Diese Defizite äußern sich mit steigender Bewegungskomplexität auch in der Bewegungsgüte. Eine weitere Störung scheint bei der Verarbeitung peripherer sensorischer Afferenzen entweder direkt oder über die Modulationen des Feedbacks vom Motocortex zu den Basalganglien vorzuliegen. Daß Parkinsonkranke eine verstärkte Abhängigkeit von visuellen Informationen zeigen, wurde wiederholt nachgewiesen (vgl. Müller \& Konczack, 1995). Diese wurde durch einen im Gegensatz zu Gesunden stärkeren Rückgriff auf Closed-loop-Steuerungen (vgl. S. 29) erklärt, die schon vor dem Bewegungsstart die Bewegung so weit vorbereitet haben, daß sie Open-loop-gesteuert ablaufen kann. Klinisch manifest wird die mangelhafte 
Verarbeitung der sensorischen Afferenzen bei der Mikropathie, dem Phänomen der immer kleiner werdenden Buchstabengröße innerhalb eines Schriftzugs.

Das Cerebellum übt über die Projektion zu verschiedenen Kernen bzw. zum Motocortex seinen Einfluß auf die Motorik aus. Hierbei sind drei Anteile zu unterscheiden, die funktional und anatomisch getrennt sind. Das Archicerebellum projiziert zu der Formatio reticularis und den Vestibulärkernen, deren Fasern einen Teil des erwähnten ventromedialen Anteils bilden und damit proximale Bewegungen des Körpers kontrollieren. An der Bewegung der Gliedmaßen ist das Paläocerebellum beteiligt, welches Fasern zum Nucleus ruber und zu den Basalganglien aufweist. Der laterale Anteil, das Neocerebellum, wirkt über den ventralen Thalamus auf den Motocortex ein und hat somit Zugang zum Tractus corticospinalis und damit zu den distalen Bewegungen der Arme und Finger sowie den proximalen Bewegungen des Körpers. Gleichgewichtsprobleme nach Kleinhirnschädigungen sind vor allem auf eine Traumatisierung dieser Strukturen des Cerebellums zurückzuführen.

Klinische Symptome nach Schädigung des Kleinhirns sind vor allem Ataxie (Koordinationsschwierigkeiten), Dysmetrie (mangelnder Bewegungsimpuls bei der Initiierung von Handlungen, zumeist überschießend), Dysdiadochokinese (Schwierigkeiten bei schnell alternierenden Bewegungen), Intentionstremor (in der Bewegung auftretender Tremor) und Dysarthrie (verlangsamte, skandierende Sprache, artikulatorische Schwierigkeiten).

Über die genaueren cerebellären Funktionen divergieren die Ansichten: Eine endgültige Klärung, ob sie 'Sitz der Koordination' sind, eine unterstützende Beteiligung an koordinativen Prozessen haben, in die Planung und Programmierung von Bewegung sowie der Transformation von räumlicher in zeitliche Information eingebunden sind, bzw. als Feedforward-Controller, Co-Prozessor oder Speicher für Bewegungsprogramme mit theoretisch $10^{11}$ adressierbaren Speichereinheiten fungieren, scheint in weiter Ferne (Müller \& Konczack, 1995, S. 673). 


\subsubsection{Die Fähigkeiten der motorischen Systeme}

Die Begriffe Kondition und Koordination als Elemente, die die motorischen Fähigkeiten konstituieren, werden aus spotrwissenschaftlicher Sicht eingeführt und erläutert. Kraft, Schnelligkeit und Ausdauer sind Bestandteile der Kondition. Bduktive und induktive Ansätze versuchen, die Steuerungsmechanismen der Koordination zu erklären. Zu den deduktiven Ansätzen gehören die beiden großen Klassen des 'Motor Approach' und des 'Action Approach', innerhalb der induktiven sind die sieben Komponenten nach Blume (s. u.) weitestgehend bekannt.

Die von außen objektivierbare Leistung der beschriebenen motorischen Systeme (s. o., S. $17 \mathrm{ff}$.$) wird in den Sportwissenschaften4 traditionell in die Bereiche der konditionellen$ und koordinativen Fähigkeiten eingeteilt (Blume, 1981), innerhalb derer die konditionellen neben Ausdauer, Kraft und Schnelligkeit zum Teil auch noch die Beweglichkeit umfassen (vgl. Letzelter, 1987, S. 178; für einen grundlegenderen Einblick: Gropler \& Thieß, 1976). Die ,,... konditionellen Fähigkeiten ... [sind] überwiegend durch energetische Prozesse, die koordinativen durch die Prozesse der Bewegungssteuerung und -regelung, d. h. informationelle Prozesse, bestimmt“ (Blume, 1981, S. 17). Diese Aussage ist jedoch vor allem hinsichtlich der Energiebereitstellung und innerhalb derer besonders für die Ausdauer gültig. Schnelligkeit ist in hohem Maße durch zentralnervöse Prozesse bestimmt. Bezüglich der Kraft ,... konnte gezeigt werden, daß Motocortexneurone, die in die Pyramidenbahn projizieren, zeitlich vor der Muskelaktivität entladen und daß deren Entladungsfrequenz hoch mit der Kraftentwicklung im Muskel korreliert“ (Schnitzler \& Freund, 1995, S. 689).

Kraft gilt als die „,... Fähigkeit des Muskels, sich trotz großen Widerstandes, aber ohne Zeitvorschrift zusammenzuziehen, bzw. gegen diesen das Ausmaß der gewollten Verkürzung zu halten“ (Deschka, 1961, S. 13). Nach einer anderen Definition ist „... Kraft im Sinne der motorischen Grundeigenschaften ... diejenige Eigenschaft des Menschen, mit deren Hilfe er eine Masse (seinen eigenen Körper oder ein Sportgerät) bewegt, seine Fähigkeit, einen äußeren Widerstand zu überwinden oder ihm durch Muskeleinsatz entgegenzuwirken“ (Pöhlmann \& Dressel, 1979, S. 90). Wie aber entwickelt ein lebender Organismus diese Kraft? Auf unterster Ebene, der Ebene der Muskelfasern, findet ein chemischer Prozeß statt. Wichtig sind hierbei die Aktin- und Myosinfilamente, Bestandteile der Muskelfasern, die aneinander vorbeigleiten und damit die Verkürzung des Muskels bewirken. Nach Anla-

${ }^{4}$ „Die Rede von einer Sportwissenschaft ist angesichts der Differenzierung praktizierter sportwissenschaftlicher Forschung nicht aufrecht zu erhalten. Der Begriff im Singular trägt nicht. Er gaukelt eine Identität vor, die nicht nur faktisch nicht gegeben ist, sondern die auch von der Sache her nicht möglich ist, ja nicht einmal angezielt werden sollte. Hierfür spricht, daß die Eigenständigkeit einer in sich identischen, gegenüber anderen Wissenschaften abgrenzbaren Sportwissenschaft weder von einem ihr eigenen und und in sich präzise definierbaren Gegenstand aus gewonnen werden kann noch von einer ihr und nur ihr eigenen Erkenntnismethodik, die in sich ebenso stringent als eigenständig begründet werden können muß“ (Lieber, 1988, S. 125; kursiv im Original). 
gerung eines Calcium-Ions verliert das in den Filamenten enthaltene Protein Troponin seine hemmende Wirkung auf die Aktin-Myosin-Verbindung. Durch die Spaltung des am Myosinkopf gebundenen ATP (Adenosintriphosphat) und Abgabe eines Phosphat-Ions kann der Myosinkopf „kippen“ (aus der 90-Grad-Stellung in eine 50-Grad-Stellung) und die Aktinund Myosinfilamente gleiten aneinander vorbei. Nach Abgabe des verbleibenden ADP (Adenosindiphosphat) kippt der Kopf noch etwas weiter in seine Endstellung (45-GradStellung), wodurch das Gleiten beendet wird. Der übriggebliebene Aktin-Myosin-Komplex ist stabil ('Rigorkomplex') und kann nur durch eine erneute Bindung von ATP an die Myosinköpfe wieder gelöst werden. Nach dieser Bindung löst sich die Aktin-MyosinVerbindung, und der Kopf kehrt in seine Ausgangslage zurück, bereit, eine neue 'Ruderbewegung' auszuführen (vgl. Silbernagl \& Despopoulus, 1991, S. 38 f.). Hieraus ergibt sich die Wichtigkeit des ATP für jede Form der Kraftäußerung; jede Erholung oder Belastung muß also unter dem Gesichtspunkt der Bereitstellung des ATP betrachtet werden. Die Initiierung dieses Prozesses geschieht durch ein Aktionspotential, einen kurzzeitigen Stromfluß, der die motorische Einheit innerviert. Als motorische Einheit bezeichnet man eine motorische Nervenzelle (Alpha-Motoneuron) mit ihrer efferent leitenden, langen Nervenfaser (Axon) und dem von ihr versorgten Kollektiv an Muskelfasern. Diese Muskelfasern, die von einem einzelnen Motoneuron innerviert werden, treten auch gemeinsam in Aktion. Als Reaktion auf äußere oder innere Reize gelangen Erregungen zu den im Rükkenmark gelegenen Alpha-Motoneuronen, wo sie auf der Grundlage elektrischer Vorgänge zu Aktionspotentialen führen, die entlang des Axons bis zur motorischen Endplatte, der Verbindungsstelle zwischen Axonende und Muskelfaser, elektrisch fortgeleitet werden. Dort wird die elektrische Erregungsleitung kurzzeitig durch eine chemische Erregungsübertragung ersetzt. Von der Endplattenregion der Muskelfaser aus setzt sich die elektrische Erregung in Richtung zu beiden Polen fort und führt schließlich aufgrund der oben beschriebenen Zusammenhänge zur Kontraktion der Muskelfaser. Zwischen dem Auftreten des Aktionspotentials der Muskelfaser und dem Beginn der Kontraktion liegt ein Zeitintervall von etwa $10 \mathrm{~ms}$.

Die isometrische Kontraktion stellt jene Arbeitsweise des Muskels dar, bei der, bei maximaler Muskelanspannung, der Abstand zwischen Ursprung und Ansatz des Muskels nicht verändert wird. Es kommt in diesem Fall zu keiner Bewegung und daher zu keiner mechanischen Arbeit. Der äußere Widerstand wird nicht überwunden. Die isotonische Kontraktion dagegen ist jene Arbeitsweise des Muskels, bei der der Abstand zwischen Ursprung und Ansatz des Muskels verändert wird. Die isotonische Kontraktion überwindet den äußeren Widerstand. Es kommt zu einer Bewegung. 
Die Erscheinungsweise der Kraft wird in einige Teilaspekte unterteilt, die eine differenziertere Beurteilung ermöglichen.

Die Absolutkraft berechnet sich aus dem Muskelquerschnitt. Da aber willkürlich nur eine bestimmte Faseranzahl gleichzeitig innerviert werden kann, ist die Maximalkraft die „... höchste Kraft, die das Nerv-Muskelsystem bei maximaler willkürlicher Kontraktion auszuüben vermag" (Harre, 1979, S. 134). Hieraus ergibt sich beispielsweise, daß ein Krafttraining nicht nur darauf abzielen sollte, den Querschnitt als solchen zu erhöhen, sondern auch die intra-muskuläre Koordination zu verbessern. In bezug auf die Versorgung (Ausmaß der Kapillarisierung) ist dies sogar die ökonomischere Alternative.

Geht es darum, möglichst schnell Kraft zu entwickeln, d. h. eine hohe Kraftzunahme pro Zeit, spricht man von Explosivkraft. Schnellkraft ist die Fähigkeit, Widerstände in sehr kurzer Zeit zu überwinden. Die Widerstandsfähigkeit gegen Ermüdung wird dann wichtig, wenn es gilt, Kraft über längere Zeit aufrecht zu erhalten: Es handelt sich hierbei um die Kraftausdauer (auch hier zeigt sich die enge Verflechtung der konditionellen Fähigkeiten, da die Trennung von Kraft, Schnelligkeit und Ausdauer indirekt immer wieder aufgehoben wird). Startkraft schließlich ist die Fähigkeit, gleich zu Beginn der Kontraktion hohe Kraftwerte zu erzielen (vgl. Harre, 1979).

Schnelligkeit wird als ,... die Fähigkeit definiert, bei der aufgrund von Beweglichkeit der Prozesse des Nervensystems und des Muskelapparates Bewegungen in optimalen Zeiteinheiten durchgeführt werden können“ (Martin, 1979, S. 104). Mehr noch als die anderen konditionellen Fähigkeiten ist die Schnelligkeit als ein Komplexgeschehen zu betrachten. Im Gegensatz zu der Kraft beispielsweise hat sie keine eindeutige physiologische Grundlage, sie setzt sich vielmehr aus den Faktoren Reaktionszeit, Bewegungsgeschwindigkeit und Bewegungsfrequenz zusammen.

Reaktionsschnelligkeit gilt als die Fähigkeit, auf einen einfachen Reiz hin so schnell wie möglich zu reagieren. Hierbei ist, neben der basalen Reaktionszeit, die Verteilung der FTFasern sehr entscheidend, da sie die Frequenz der Innervation bestimmen; ein weiterer entscheidender Faktor, neben dem nicht ganz so wichtigen Muskelquerschnitt, ist hier die inter- und intramuskuläre Koordination. Ausdauer ist der Ermüdungswiderstand (s. u.), so kommt es bei der Schnelligkeitsausdauer vor allem darauf an, die Geschwindigkeit über eine bestimmte Zeit aufrecht zu erhalten. Bei Sprintern ist die maximale Geschwindigkeit nach etwa 20 bis 30 Metern erreicht. Ihnen gelingt es nur selten, bis zur 100-Meter-Linie ohne Leistungsabfall durchzuhalten. Ursachen hierfür sind zum einen die Ermüdung des 
Nervensystems durch die hohe Belastungsfrequenz, zum anderen der hohe Energieaufwand pro Zeiteinheit und schließlich die Übersäuerung des Muskels durch die Laktatanhäufung.

Ausdauer, leistungsbestimmender Faktor im Langstreckenlauf oder in der Fahrradergometrie (Schürch, 1987, S. 38) und als nicht isoliert existierende Fähigkeit „,... immer an die Inanspruchnahme von unterschiedlichen Anteilen an Kraft, Schnelligkeit und Koordination gebunden“ (Neumann \& Schüler, 1994, S. 20; Hervorhebungen im Original), ermöglicht die Aufrechterhaltung einer Leistung über eine bestimmte Zeit (vgl. Kwiatkowski, 1987, S. 47), sie ist die ,... psycho-physische Widerstandsfähigkeit gegen Ermüdung“ (Hegner, 1988, S. 126).

Nach Hollmann und Hettinger (1980) wird die Ausdauer in eine lokale und eine allgemeine Ausdauer eingeteilt, wobei die allgemeine gleichzeitig die Grundlagenausdauer darstellt. Beide Ausdauerformen werden hinsichtlich der Energiebereitstellung in 'aerob' und 'anaerob' unterteilt. Diese Untergruppen erfahren jeweils noch eine Differenzierung in 'statisch' und 'dynamisch'. Die häufig vorgenommene Einteilung in Kurz-, Mittel- und Langzeitausdauer ist sehr unscharf, da die Bereiche nur willkürlich voneinander abgegrenzt werden können. Für die Kurzzeitausdauer wird gewöhnlich ein Bereich von 45 Sekunden bis zu etwa 2 Minuten angegeben (Harre, 1979, S. 157; Mühlfriedel, 1979, S. 81), die Mittelzeitausdauer findet in einem Bereich von 2 bis etwa 10 Minuten statt (Harre, 1979, S. 157; Mühlfriedel, 1979, S. 57), teilweise auch zwischen 10 und 30 Minuten (Hollmann \& Hettinger, 1980, S. 348). Jede längere Zeitspanne kann als Langzeitausdauer angesehen werden (ebd.; siehe auch Fußnote 5, S. 28).

Die physiologische Betrachtungsweise der ersten Stufe der Langzeitausdauer ${ }^{5}$, die häufig bei der Fahrradergometrie von Interesse ist, wird durch folgende Eckpunkte abgesteckt: Muskel- und Leberglycogen, deren Depots selbst bei intensivster Leistung nur zu einem Drittel ausgeschöpft werden, sind die wichtigsten Energielieferanten; die energetische Absicherung der Leistung erfolgt, bei hauptsächlicher Rekrutierung der ST-Muskelfasern, aerob, die maximale Sauerstoffaufnahme wird dabei zu 90 bis $95 \%$ ausgenutzt (vgl. Neumann \& Schüler, 1994).

Die koordinativen Fähigkeiten als „,... relativ verfestigte und generalisierte (verallgemeinerte) Besonderheiten des Verlaufs der Steuer- und Regelprozesse“ (Zimmermann, 1986, S. 211) gelten als die die menschliche Bewegung steuernden und

\footnotetext{
${ }^{5}$ Nach Peters und Phillip (1985) etwa 15 min (S. 497).
} 
ordnenden bzw. organisierenden Größen. Auch wenn diese begriffliche Annäherung auf einem höheren Abstraktionsniveau gültig sein mag, gestaltet sich die konkrete Definition schwierig und kann nur auf verschiedenen Ebenen stattfinden. Aus Sicht der Neurophysiologen handelt es sich um das Zusammenspiel von Muskelarbeit und beteiligten neuronalen Prozessen, die Biomechanik stellt die räumliche, zeitliche und kräftemäßige Ordnung menschlicher Bewegungsvollzüge in den Mittelpunkt, für Handlungstheoretiker ist Bewegungskoordination immer eine komplexe Leistung der Gesamtpersönlichkeit und der Sportpraktiker schließlich schließt aus mehr oder weniger standardisierten Beobachtungen äußerlich sichtbarer Bewegungsmerkmale auf interne motorische Leistungsfaktoren (vgl. Klein, 1987). Im Verbund der Theorien, die sich um Klärung der motorischen Steuerung bemühen, lassen sich zwei grundlegende Theorierichtungen identifizieren. Deduktive Ansätze versuchen, neurophysiologische, anatomische, biochemische und kybernetische Erkenntnisse zu einem möglichst weitreichenden Modell der Bewegungsregulation zu integrieren, während Vertreter der induktiven Ansätze den Sportpraktikern vergleichbar vorgehen, indem sie von äußeren, beobachtbaren Merkmalen auf zugrundeliegende Fähigkeiten schließen. Dem oben beschriebenen funktionellen bzw. lokalisatorischen Ansatz steht nun ein phänomenologischer gegenüber, bei dem der Versuch der Lokalisation der beschriebenen Systeme in den Hintergrund tritt. Dieser Konflikt ist innerhalb der psychologischen Theorienbildung häufig zu beobachten, da bereits viele wissenschaftlich gesicherte, psychologische Phänomene beschrieben werden können, für die aber Neurophysiologie bzw. psychologie noch kein anatomisches Substrat gefunden haben (vgl. S. 49).

$\mathrm{Zu}$ den deduktiven Ansätzen gehören neben den klassischen Reiz-Reaktions-Theorien (vgl. Bower \& Hilgard, 1983) und den Open-loop-Theorien vor allem die feedbackgestützten, kybernetischen Modelle wie die Closed-loop-, Schema- und GMP-Theorie (nach Bund, 1992; Klein, 1987).

In seiner 1971 als Reaktion auf die Unzulänglichkeit der Open-loop-Modelle (Adams, 1989, S. 71) veröffentlichten Closed-loop-Theorie des motorischen Lernens geht Adams im Unterschied zu bestehenden Regelkreismodellen von zwei unabhängigen Gedächtnisspeichern aus. Im ersten, dem sogenannten Wiedergabespeicher ('memory-trace'), sind einfache motorische Programme enthalten, die die Bewegung initiieren und ihre anfängliche Richtung festlegen. Darüber hinaus müssen diese Kurzprogramme die ersten Bewegungssequenzen steuern (s. u.). In einem zweiten Gedächtnisbereich, dem Wiedererkennungsspeicher ('perceptual trace'), sind die Sollwerte der Bewegungen enthalten. Sie werden vor Bewegungsbeginn abgerufen und bestehen aus den erwarteten sensorischen Konsequenzen 
der geplanten Bewegung, d. h. denjenigen Rückmeldungen, die man bei völlig exakter Ausführung erhalten würde. Da Adams (1989) den Gültigkeitsbereich seiner Theorie auf einfache, langsam durchgeführte Positionierungsaufgaben beschränkt, beziehen sich die Sollwerte in der Regel auf die korrekte Endposition der bewegten Körperextremität. Während der Bewegung nutzt der Ausführende diesen 'sensorischen Sollwert' der Wahrnehmungsspur und die tatsächlich einlaufenden Rückmeldungen (Istwert) zu einem kontinuierlichen Soll-Istwert-Vergleich. Signalisiert die Wahrnehmungsspur eine Differenz zwischen Sollwert und Istwert werden solange Korrekturen vorgenommen, bis die einzelnen Körperextremitäten ihre geplanten Endpositionen erreicht haben, an denen die Soll-Istwert-Differenz gleich Null ist. Auf diese Weise kommt es gewissermaßen zu einer allmählichen Annäherung an das vorgegebene Bewegungsziel. Zu Beginn des motorischen Lernprozesses, bei geringer Bewegungserfahrung, sind die Sollwerte der Wahrnehmungsspur im Wiedererkennungsspeicher allerdings noch wenig zuverlässig. Sie bilden sich auf der Grundlage von vorangegangenen Versuchen und den dabei erhaltenen Rückmeldungen. In diesem frühen Stadium repräsentiert der Sollwert somit noch nicht unbedingt die gewünschte Zielbewegung, der Fehler wird aber nicht korrigiert, da ja der (falsche!) Sollwert erreicht wird. In dieser Phase sind deshalb externe Rückmeldungen über Fehler durch Außenquellen notwendig, um Verbesserungen zu erzielen. Mit zunehmender Zahl der Bewegungswiederholungen wird die Wahrnehmungsspur gefestigter, genauer und der interne Fehlererkennungsmechanismus damit zuverlässiger. Auf dieser Grundlage werden von Adams (1989) sogar Lernfortschritte ohne Rückmeldung vorhergesagt, weil der auf dem exakten Sollwert basierende Soll-Istwert-Vergleich alle Fehler (intern) aufdeckt. Motorisches Lernen nach der Closed-loop-Theorie besteht also im wesentlichen in der Entwicklung der Fähigkeit, Fehler erkennen und korrigieren zu können. Die Annahme zweier Gedächtnisspeicher hat folgende Gründe: Zum einen kann die Vergleichsfunktion der Wahrnehmungsspur (und damit die Bewegungsführung) erst dann zum Tragen kommen, wenn Rückmeldungen vorhanden sind, d. h. erst kurz nach Bewegungsbeginn. Deshalb muß zunächst ein einfaches Programm die Bewegung auslösen und in den ersten 120 bis 200 ms steuern. Zum anderen existieren damit getrennte Gedächtnisinstanzen für die Bewegungsauslösung (Wiedergabespeicher) und die Bewegungsüberprüfung (Wiedererkennungsspeicher). Auf diese Weise können nicht nur Fehler im Programmablauf erkannt werden, sondern auch Fehler in der Programmauswahl (Bund, 1992).

Das Schemakonzept entstand in der Neurophysiologie, wurde aber bald von der Psychologie übernommen, um die Verhaltensvielfalt ('versality') zu erklären (Adams, 1989). Schmidt (1975) nahm diese Konzepte - motorisches Programm und Schema - auf und inte- 
grierte sie zu einer Theorie. Die Schema-Theorie (Schmidt, 1975) erklärt den Erwerb, die Speicherung und die Wiedergabe komplexer Bewegungen; dabei umfaßt sie (im Gegensatz $\mathrm{zu}$ früheren Theorien) sowohl schnelle, ballistische Bewegungen als auch langsame Positionierungsaufgaben. Im Mittelpunkt der Schema-Theorie steht zum einen die Annahme generalisierter motorischer Programme (GMP) und zum anderen die Verwendung des Schema-Begriffs. Die Idee generalisierter motorischer Programme besagt, daß ein motorisches Programm nur einige wenige Merkmale einer Vielzahl ähnlicher Bewegungen enthält, die einer Bewegungsklasse angehören (s. u.). Diesen invarianten Programmelementen werden dann mit Hilfe der motorischen Schemata variable Bewegungsparameter hinzugefügt, die die Details der Bewegungsausführung festlegen. Dabei unterscheidet Schmidt (1975) ein Recall-Schema, das für die Auswahl des generalisierten motorischen Programms und die Steuerung sehr schneller Bewegungen verantwortlich ist sowie ein Recognition-Schema, das die sensorische Informationsverarbeitung übernimmt, indem es das aktuelle Feedback mit den erwarteten Rückmeldungen vergleicht und so langsame Bewegungen kontrolliert. Für jedes GMP wird jeweils ein Recall- und ein Recognition-Schema gebildet. Damit wurden in der Schema-Theorie zum ersten Mal zentrale Programmierungsund periphere Feedbackvorgänge gemeinsam berücksichtigt: Schnelle Bewegungen stehen ausschließlich unter der Kontrolle eines motorischen Programms, langsame Bewegungen bieten dagegen durch einen Soll-Istwert-Vergleich des Feedbacks die Möglichkeit peripherer Koordination. Dieser neue Ansatz wurde als 'Mixed Approach' bezeichnet. Die jahrzehntelange Kontroverse 'Programmsteuerung versus Regelung' galt damit als überwunden. Das Konzept generalisierter motorischer Programme bot darüber hinaus die Lösung für Fragen, die von den bisherigen Open-loop- und Closed-loop-Modellen nicht beantwortet werden konnten (Schmidt, 1975).

Die derzeit einflußreichste Programmtheorie ist die über zehn Jahre später veröffentlichte Theorie generalisierter motorischer Programme (Schmidt, 1988; siehe auch Bund, 1992). Die in ihr geäußerten Vorstellungen über den Aufbau und die Struktur motorischer Programme sind in den letzten Jahren von vielen Motorikforschern aufgegriffen und in wesentlichen Aspekten empirisch überprüft worden.

Ausgehend von der Beobachtung, daß menschliche Willkürbewegungen einerseits konstante - vor allem qualitativ-topologische - Merkmale aufweisen, andererseits aber auch in ihrer Ausführung durchaus variabel gestaltet werden können, werden in der GMP-Theorie zwei Gruppen von Programmierungselementen unterschieden: Zum einen gibt es invariante, unveränderbare Bestandteile, die direkt in den motorischen Programmen festgeschrieben sind, zum anderen variable, veränderbare Parameter, die über Recall-Schemaregeln in die 
auszuführenden Rahmen- oder Grundprogramme eingelesen werden können. Die festen, unveränderbaren Programminhalte werden mit Hilfe der sogenannten Impuls-TimingHypothese beschrieben. Diese besagt, daß ein motorisches Programm 'Zeit- und Kraftinformationen' zu allen Muskeln sendet, die für die geplante Bewegung ausgewählt worden sind: „A motor program tells the muscles when to turn on, how much, and when to turn off. That is, the motor program ultimately controls force and time" (Schmidt, 1988, S. 242). Aus diesen Kennwerten (Sequencing, relatives Timing, relative Kräfte) setzt sich ein für die Bewegungsfertigkeit charakteristisches Impuls-Timing Muster zusammen, das den beteiligten Muskelgruppen genau vorschreibt, wann die Aktivität beginnen und enden soll und mit welcher Intensität der Einsatz zu erfolgen hat. Dennoch sind damit lediglich die Strukturmerkmale der Bewegung festgelegt. In dieser Form kann deshalb ein generalisiertes motorisches Programm eine Klasse von Bewegungen steuern, die ein ähnliches zeitlich-dynamisches Grundmuster aufweisen. Welche spezielle Variationsform aus dieser Klasse ausgeführt wird, hängt von der Festlegung der Parameterwerte ab. Als wichtigste austauschbare Bewegungsmerkmale werden in der GMP-Theorie die Gesamtbewegungsdauer und der Absolutkrafteinsatz genannt. Mit Hilfe der Gestaltkonstanz-Hypothese erklärt die GMPTheorie die variable Verfügbarkeit bzw. die situationsabhängige Feinprogrammierung der generalisierten motorischen Programme. Sie besagt, daß die in den motorischen Programmen enthaltenen bewegungssteuernden Impuls-Timing-Muster in zeitlicher (horizontaler) bzw. dynamischer (vertikaler) Hinsicht proportional gedehnt oder gestaucht werden können, ohne daß sie ihre charakteristische Form verändern. Die Dehnung bzw. Stauchung des Impuls-Timing Musters ist auf metrische Parameter zurückzuführen, die variabel in das motorische Programm eingelesen werden: Die Bewegungsdauer und der Gesamtkrafteinsatz. Die Spezifikation dieser variablen Bewegungsparameter orientiert sich an den aktuellen Ausgangsbedingungen der Bewegung, die Zeit- und Kraftrelationen des Impuls-Timing Musters bleiben aber über die Variation von Bewegungsdauer und Krafteinsatz hinaus konstant. So kann ein Basketballspieler von variierenden Positionen Zielwürfe ausführen, wobei die Grundstruktur der Wurftechnik stets dieselbe ist. Aus GMP-theoretischer Sicht wird in diesem Fall immer das gleiche generalisierte Programm nur mit unterschiedlichen Parameterwerten für den Krafteinsatz realisiert.

Kritisch zu den Ansätzen des deduktiven Verständnisses äußert sich Wollny (1991; siehe Blockzitat S. 34), der innerhalb dieser jedoch eher den ökologischen Systemmodellen ('Action Approaches') den Vorzug gibt. Diese betonen die Ganzheitlichkeit und die aktive, zielgerichtete Auseinandersetzung mit den unmittelbaren Umweltanforderungen und den 
zahlreichen auf das Individuum einwirkenden emotionalen, individuellen sowie sozialen Faktoren und stellen damit die aktuelle Gegenposition zu motorischen Programmtheorien dar (Wollny, 1991). Eine entscheidende Bedeutung für die Bewegungskoordination wird den visuellen Eindrücken des Handelnden beigemessen. Aktionsforscher gehen von der Annahme einer direkten wechselseitigen Kopplung von visuellen Wahrnehmungs- und motorischen Handlungsprozessen aus (Gibson, 1979). Die Bewegungskontrolle ist daher nicht ausschließlich innerhalb des Organismus anzusiedeln. Sie wird, im Gegenteil, maßgeblich durch die individuell wahrgenommenen, vielfältigen Mensch-Umwelt-Beziehungen bestimmt. Folgerichtig wird die Existenz zentral gespeicherter, autonomer Bewegungsprogramme verneint, weil diese nach Auffassung der Aktionstheoretiker unvorhersehbare externe Störungen, Veränderungen im Bereich der Schwerkraft u. ä. nur unzureichend berücksichtigen oder kompensieren können. Die Kontrolle motorischer Handlungen erfolgt hier vielmehr über selbstorganisierte und aufgabenspezifische 'Aktionssysteme', die von höheren Kommandozentren des ZNS unmittelbar vor und während der Bewegung - unter Berücksichtigung der aktuellen Umweltbedingungen - aktiviert werden. Bernstein (1967) bezeichnet diese Aktionssysteme als 'Functional Synergy'. Sie stellen koordinative Strukturen selbstregulierender funktioneller Systeme dar, die in unmittelbarer Abhängigkeit der Wechselbeziehungen zwischen Mensch, Umwelt und Bewegungsziel sowohl einfache als auch komplexe Bewegungsverläufe durch die aufgabenspezifische Kopplung von synergistisch arbeitenden Muskeln und der sie einschließenden Gelenke autonom kontrollieren. Ähnlich wie motorische Programme handelt es sich bei koordinativen Strukturen um abstrakte Formulierungen der wesentlichen invarianten Kennwerte der Bewegung. Die postulierten Invarianzen sind jedoch innerhalb höherer Strukturebenen des ZNS zu suchen und scheinen dort als qualitativ-topologische Kennwerte (räumlich-zeitlicher Verlauf der Bewegung) vorzuliegen. Es determinieren also nicht „...zentral gespeicherte Bewegungsprogramme menschliche Willkürbewegung, sondern höhere Zentren des ZNS scheinen durch einzelne neuronale Impulssignale hierarchisch tieferliegende, selbstorganisierte biologische Systeme zu aktivieren, die ihrerseits eine feststehende, aufgabenspezifische Gruppierung einzelner Muskeln autonom steuert [sic]“" (Wollny, 1991, S. 82). Die situationsadäquate Anpassung ('tuning') koordinativer Strukturen an das ausgewählte Bewegungsziel erfolgt vor und während der Bewegungsausführung durch sogenannte 'Tuning-Parameter'. Diese 'TuningParameter' beeinflussen vornehmlich metrische Bewegungsmerkmale wie die absolute Bewegungszeit und das Aktivitätsniveau der bewegungsausführenden Muskulatur. Die Parameterwerte können aufgrund der angenommenen direkten Kopplung von Wahrnehmung 
und Motorik unmittelbar aus der aktuellen Umwelt entnommen werden; die Einstellung erfolgt über niedere neuronale Schaltkreise im Rückenmark (vgl. Bund, 1992).

Eine Synthese dieser Ansätze scheint in der Akzeptanz beider zu liegen. Die Frage ist nicht, „,... welcher der beiden kontrovers diskutierten konzeptionellen Wege - Motor- vs. Action Approach - der alleinige ist, sondern unter welchen Bedingungen, [sic] welches motorische Kontrollverfahren sinnvoll und plausibel erscheint" (Wollny, 1991, S. 86; kursiv im Original). Für beide Klassen jedoch gilt:

Bis heute ist es den Aktionsforschern nicht gelungen, eindeutige empirische Nachweise ihrer generellen Annahmen über die menschliche Bewegungskontrolle zu erbringen. In diesem Punkt befinden sie sich in guter Nachbarschaft zu den diskutierten motorischen Programm- und Regelkreistheorien, die ebenfalls nur indirekte Evidenzen für die Existenz und die spezifische Funktionsweise motorischer Programme und zentral-nervöser Instanzen für periphere Sollwertschätzungen aufweisen können. (ebd.)

Gemäß der induktiven Ansätze zeigt Blume (1981) sieben Fähigkeiten auf, die nach seiner Forschung empirisch abgeleitet und beschrieben werden können: Kopplungsfähigkeit, Orientierungsfähigkeit, Differenzierungsfähigkeit, Gleichgewichtsfähigkeit, Reaktionsfähigkeit, Umstellungsfähigkeit und Rhythmisierungsfähigkeit. Bei der Identifizierung der zur Bewegungssteuerung relevanten Merkmale beschreibt er folgende (Blume, 1981, S. 19 f.):

- Zur Bewältigung bestimmter Handlungsziele sind eine mehr oder weniger große Anzahl von Teilkörperbewegungen, Einzelbewegungen oder Bewegungsphasen miteinander zweckentsprechend zu koordinieren.

- Der gesamte Körper verändert ständig mehr oder weniger umfangreich und vielgestaltig seine Position zu der ihn umgebenden Umwelt.

- Die Gesamt- und Teilbewegungen müssen oft mit einer hohen Präzision ausgeführt werden, um das Handlungsziel optimal zu erreichen.

- Sehr häufig gilt es, auf bestimmte Signale zu reagieren, d. h. zum rechten Zeitpunkt eine zweckmäßige motorische Aktion auszuführen.

- In einer Reihe von Sportarten ist ein Anpassen an oder Umstellen auf plötzlich auftretende Situationsveränderungen notwendig. 
- Der sich bewegende Körper ist entweder im Gleichgewicht zu halten oder nach großräumigen, zum Teil auch sehr schnellen Lageveränderungen in den Gleichgewichtszustand zurückzuführen.

- In einigen Sportarten liegt eine Besonderheit der Bewegungssteuerungen in der Abstimmung der Bewegung auf einen bestimmten Rhythmus, der akustisch, musikalisch oder auch visuell vorgegeben ist.

Einen anderen Zugang wählten Fleishman und seine Mitarbeiter (nach Klein, 1987, S. 195 ff.), die nach Messungen und Beobachtungen in etwa 200 standardisierten Bewegungsaufgaben $(\mathrm{N}>1000)$ ebenfalls etwa sieben allgemeine koordinative Fähigkeiten ableiteten. Diese sind motorische Steuerungsfähigkeit (allgemein), motorische Steuerungsfähigkeit der oberen Extremitäten, motorische Kombinationsfähigkeit, motorische Anpassungs- und Umstellungsfähigkeit, Fingergeschicklichkeit, Handgeschicklichkeit und Reaktionsschnelligkeit.

An den vom sowjetischen Neurophysiologen und Vater der neuropsychologischen Grundlagenforschung A. R. Luria entwickelten Modellvorstellungen und Erkenntnissen überprüfte Hirtz (1964) die von ihm bestimmten koordinativen Fähigkeiten. Diese, als sportliche Gewandtheit bezeichnet, sind: Anpassungsvermögen, Orientierungsvermögen, Kombinationsvermögen, Geschicklichkeit, Wendigkeit, Gleichgewichtsvermögen, Steuerungsvermögen und Reaktionsvermögen.

Für die induktiven Ansätze muß zusammenfassend festgestellt werden, daß trotz intensiver Forschungsbemühungen noch kein Begriffssystem koordinativer Fähigkeiten geschaffen werden konnte, welches der klaren, weithin akzeptierten Differenzierung der Kondition vergleichbar wäre. Die Behauptung von Mattausch [1973], daß wir in der wissenschaftlichen Durchdringung der koordinativen Leistungsfaktoren weitgehend am Anfang stehen, gilt deshalb wohl auch heute noch. (Klein, 1987, S. 198) 


\subsection{Der Verlust der motorischen Fähigkeiten}

Hemiplegie ist die wichtigste motorische Folge eines Schlaganfalls. Die Folgen zentraler Läsionen sind Verlust der Feimotorik, Masseninnervationen, Minderung der groben Kraft, spastische Tonuserhöhung und Auslösbarkeit pathologischer Reflexe. Im Cortex sind nicht Muskeln, sondern Bewegungen repräsentiert. Als Folge von Läsionen wurden schon früh vorübergehende Schwächen, Verlangsamung und Armut aller Bewegungen auf der kontralateralen Seite bschrieben.

Für den Verlust der koordinativen Fähigkeiten gibt es vielfältige Ursachen. Hier soll vor allem jene erwähnt werden, die im Rahmen der neurologischen Rehabilitation eine herausragende Stellung einnimmt: Die Hemiplegie bzw. Hemiparese als Syndrom, das auf eine Schädigung des höheren ZNS-Bereichs zurückzuführen ist. Schädigungen, die hauptsächlich auf eine Verletzung des Rückenmarks zurückzuführen sind oder zu dem Formenkreis der entzündlichen/chronischen/progredienten Krankheiten gehören (Encephalomyelitis disseminata, Poliomyelitis, Paraplegie etc.), werden im Kontext dieser Arbeit nicht behandelt.

Zu den Hauptsymptomen nach zentralen Läsionen zählen der Verlust der Feinmotorik, Masseninnervationen, die Minderung der groben Kraft, spastische Tonuserhöhung, das Nichtvorhandensein neurogener Muskelatrophien, die Auslösbarkeit pathologischer Reflexe bei gesteigerten Eigen- und geschwächten Fremdreflexen sowie eine qualitativ unveränderte elektrische Erregbarkeit (Poek, 1994, S. 92).

Eine Schädigung des Tractus corticospinalis durch Läsionen im Gyrus praecentralis, der Pons oder der Pyramide führt zu einer schlaffen Parese mit Beeinträchtigung der Feinmotorik, pathologischen Reflexen und den bereits erwähnten Massenbewegungen. Hieraus ergibt sich ein Hinweis für die Funktion des Hirnstamms: Der Betroffene wird durch den Ausfall auf das motorische Funktionsniveau eines Kindes unter zwei Jahren zurückversetzt, bei dem die Motorik noch ausschließlich unter der Steuerung dieser Hirnstammsysteme steht (siehe S. 18). Sind Systeme des Hirnstamms bzw. der pyramidalen Bahnen gestört, kommt es vor allem zu Beeinträchtigungen der Haltefunktionen, während die distalen Funktionen und die Feinmotorik weitgehend funktionstüchtig bleiben (Poek, 1994, S. 91).

Auf ihrem Weg zum Rückenmark bündeln sich die Pyramidenbahnen und verlaufen eng benachbart in der Capsula interna, die auf der einen Seite von Thalamus und Nucleus caudatus und auf der anderen von Putamen und Pallidum begrenzt wird, so daß Läsionen an dieser Stelle zu einer Schädigung aller Pyramidenfasern einer Hemisphäre führen können. Zusätzlich sind sie an dieser Stelle besonders wahrscheinlich, da die A. lenticulostriata, die unmittelbar im Bereich der Capsula interna liegt, in einem nahezu rechten Winkel vom zuführenden Stamm der A. cerebri media abgeht und so von atheromatösen Veränderungen sehr häufig betroffen ist. Da die Kapsel aber auch cortico-striäre, cortico-thalamische, corti- 
co-rubale, cortico-oliväre und cortico-retikuläre Bahnen enthält, führen dort lokalisierte Läsionen zumeist zu dem Syndrom der spastischen Hemiplegie (vgl. S. 22).

Die Hemiplegie im allgemeinen ist eine der motorischen Hauptfolgen des Schlaganfalls (siehe Kap. 2.1.1, S. 12). Verlust der Willkürmotorik, Tonusveränderungen und veränderte Reflexe sind die wichtigsten Merkmale (Kolb \& Wishaw, 1993, S. 136 ff.). In den meisten Fällen ist die Hemiplegie eine Folge der Unterversorgung durch die mittlere Hirnarterie, der A. cerebri media, die den größten Teil des primär-motorischen Areals versorgt. Durch sie kommt es zumeist zu einer schlaffen Lähmung der zu der betroffenen Gehirnhälfte contralateral gelegenen Körperseite, begleitet von dem Verlust der Willkürmotorik. Nach einiger Zeit wandelt sich das Bild, die schlaffe Lähmung weicht einer spastischen, die von einer Hyperreflexie begleitet ist und sich vornehmlich auf die Antischwerkraftmuskulatur bezieht. Die Spastik bewirkt, daß einzelne Extremitäten wieder genutzt werden können. Dies geschieht beispielsweise bei den Beinen, die dank ihrer säulenartigen 'Erstarrung' zur Fortbewegung gebraucht werden können; gleichzeitig bewirkt jedoch die neue funktionale Gebundenheit auch größere Schwierigkeiten bei der Vermittlung physiologischer Bewegungsmuster, dem Ziel der krankengymnastischen Therapien. Die Spastik entsteht nicht zufällig in einzelnen Muskeln, vielmehr unterstützt sie den Körper in sinnvoller Weise gegen die Schwerkraft. Zusätzlich ist sie, im Gegensatz zu den Phänomenen, die in Verbindung mit der Parkinsonschen Krankheit auftreten (siehe S. 23), unidirektional, ihr Widerstand ist bei schnellen Bewegungen größer als bei langsamen und schließlich zeigt sie die Charakteristik, die als Klappmessereffekt bezeichnet wird: Der Widerstand gegen eine von außen erzwungene Bewegung nimmt erst zu, dann bricht er plötzlich zusammen.

Sind weiter distal gelegene Regionen von der Minderdurchblutung betroffen, zeichnet sich mit den dazugehörigen Sensibilitätsstörungen das klinische Bild der Fazialis- bzw. der Brachiofazialisparese ab. Der proximale Verschluß der A. cerebri anterior ist klinisch nicht vom A.-cerebri-media-Infarkt zu unterscheiden. Erst wenn der Verschluß eher distal liegt, kommt es zu einer beinbetonten Hemiparese. Eine handbetonte Hemiparese ist Folge der Minderdurchblutung der A. cerebri posterior, die aber nicht aus der A. cerebri media, sondern aus der A. basilaris entspringt. Ein Verschluß der A. basilaris und der A. vertebralis ist für Störungen in subcortikalen Anteilen verantwortlich. Dabei betroffen sind das Mittelhirn, das Kleinhirn und der Hirnstamm. Hemi- oder Tetraparesen, halbseitige Hyperkinese und Ataxie können Ausdruck dieser Störung sein (vgl. Poek, 1994).

Der Verlust der koordinativen Fähigkeiten ist eine direkte Folge der zentralen Läsion, da in den Arealen des motorischen Cortex „,... nicht Muskeln, sondern Bewegungen repräsen- 
tiert ...“(Poek, 1994, S. 91) sind. Dies gilt ebenso für Teile der konditionellen Fähigkeiten; ein Kraft- und Schnelligkeitsverlust ist einer zentralen Lähmung immanent. Der Verlust der konditionellen Fähigkeiten variiert in Abhängigkeit von den jeweils betrachteten Komponenten. Im Mittelpunkt steht jedoch die cortikale Aktivität, die das Alpha-Motoneuron als den ,... räumlichen und zeitlichen Summationspunkt peripher-afferenter, deszendierender und interneuronaler Einflüsse, die sowohl erregend als auch hemmend wirken können“ (Hummelsheim, 1996 b, S. 144), durch die zentralnervöse Schädigung mit verringertem oder erhöhtem, insgesamt aber im Vergleich zu dem Zeitpunkt vor der Schädigung zumindest verändertem Aktivitätsmuster innerviert.

Zusammenfassend kann für die konditionellen Fähigkeiten folgendes gesagt werden: In bezug auf die Kraft ist es die eingeschränkte Fähigkeit, motorische Einheiten zu rekrutieren, bei einer gleichzeitig veränderten Aktivierungssequenz (Schlichte, 1990), welche zu einer Schwächung bis hin zu einer Parese des Muskels bzw. zu einer Verlangsamung der Muskelaktivität führt. Als Folge von Läsionen im Bereich des prämotorischen Cortex wurden schon früh vorübergehende Schwächen, Verlangsamung und Armut aller Bewegungen auf der kontralateralen Seite beschrieben (Foerster, 1909). An Primaten konnte gezeigt werden, daß die Entladungsfrequenz von Neuronen des Motocortex hoch mit der Kraftentwicklung im Muskel korreliert (Evarts, Fromm, Köller \& Jennings, 1983). Eine Verlangsamung von Bewegungen ist zum einen auf eine verschlechterte intramuskuläre Koordination zurückzuführen, zum anderen auf eine niedrigere Innervationsfrequenz; lokalisatorisch wird dies mit Schädigungen im Cerebellum und der supplementärmotorischen Area assoziiert (Schnitzler \& Freund, 1995). Die Ausdauer wird bei den in dieser Betrachtung vornehmlich behandelten motorischen Störungen vor allem indirekt beeinträchtigt, da durch Kompensationsstrategien oder ineffektiveren Bewegungen nach der Schädigung mehr Energie benötigt wird, worauf sich der Körper gerade nach langen Liegezeiten innerhalb des Rehabilitationsprozesses erst einstellen muß. Die Liegezeiten führen durch die Schonung zu einer Besserung der allgemeinen Beschwerden, aufgrund der mangelnden Trainingsreize aber zu einer Verminderung der Kraft und der Leistungsfähigkeit des HerzKreislauf-Systems. Eine direkte Schädigung der vegetativen Regulationszentren ist selten und vor allem bei schweren Hirnkontusionen zu beobachten, bei der die vegetativen Regulationszentren im Zwischenhirn betroffen sind. 


\subsection{Die Wiederherstellung der motorischen Fähigkeiten}

Die Wiederherstellung der motorischen Fähigkeiten läßt sich zum einen auf allgemeine Faktoren zurückführen, die auch die Wiederherstellung anderer Funktionen bewirken, deren Ausfall auf eine Schädigung des ZNS-Gewebes zurückzuführen ist. Zum anderen sind spezifische Prozesse beteiligt, die in den nachfolgenden Kapiteln (Kap. 2.1.3.2.2.2, S. 43 und Kap. 2.1.3.2.2.2, S. 43) behandelt werden.

\subsection{Allgemeingültige Mechanismen der zentralnervösen Funktionswiederhe- stellung}

Funktionelle und strukturelle Umorganisation bilden ein Grundprinzip der Funktionsweise des Gehirns. Zentralnervöse Plastizität wird durch Sprouting, Vikariation, Demaskierung stiller Synapsen, Long-Term-Potenzierung, Diaschisis, mit tiple Kontrolle und Redundanz ermöglicht. Für die motorische Wiederherstellung ist besonders die Long-TermPotenzierung von zentraler Bedeutung.

Eine frühere Einschätzung, derzufolge die neuronalen Strukturen unveränderbar festgelegt sind, gilt nach heutigen Erkenntnissen als nicht mehr zutreffend. Vielmehr scheint die „... funktionelle und strukturelle Umorganisation ... ein Grundprinzip der Funktionsweise des intakten ebenso wie des umschrieben geschädigten Gehirns [zu] bilden“ (Hummelsheim, 1996 a, S. 134). Ebenso damals wie heute ist unumstritten, daß sich in einem ausgereiften Gehirn eine Regeneration total zerstörten Nervengewebes im Sinne einer Zellneubildung nicht mehr ereignen kann. Damit findet eine Erholung im eigentlichen Sinne - auf der Ebene der beteiligten Neuronen - nicht statt. Die Mechanismen, in denen sich die zentralnervöse Plastizität ausdrückt, werden durch Begriffe wie Sprouting, Vikariation, Demaskierung stiller Synapsen, Long-Term-Potenzierung, Diaschisis, multiple Kontrolle und Redundanz umschrieben. Ein Zusammenhang mit der Funktionswiederherstellung ist im kausalen Sinne jedoch nicht nachweisbar, sicher ist allenfalls, daß die Entwicklung bzw. Erholung der motorischen Fertigkeiten mit denen im folgenden angesprochenen Mechanismen zeitlich parallel verläuft.

Das Sprouting wurde gerade in Verbindung mit Tierversuchen häufig beschrieben und meint „,... das Aussprossen erhaltener Axone mit nachfolgender Neubildung synaptischer Kontaktstellen“ (Hummelsheim, 1996 a, S. 134). Hierbei sind zwei Formen zu unterscheiden: Das reaktive Sprouting, bei dem unversehrte benachbarte Axone aussprossen, und das kollaterale, bei dem die Aussprossung vom erhaltenen Axonstumpf ausgeht. Nach Seitelberger (1968) ist die Bedeutung des Sproutings hinsichtlich der Funktionswiederherstellung jedoch nur von untergeordneter Wichtigkeit. Zum einem fehlen im ZNS die Schwannschen Zellen, die bei der Axonregeneration im peripheren Nervensystem die Leitposten für die 
aussprossenden Axone darstellen und diese zu den Zielzellen für die synaptische Verbindung führen, zum anderen bildet das Narbengewebe (vgl. S. 14) gewissermaßen eine mechanische Barriere, welche die Zielgebiete von den aussprossenden Axonen trennt und das Zusammenkommen von Axon und zu bildender Synapse an der Zielzelle verhindert. Es gibt aber auch Hinweise, daß trotz der Barriere Kollateralen sehr zielsicher ihren Weg um das geschädigte Gebiet herum fanden (Kolb \& Wishaw, 1993). Eine andere Schwierigkeit tritt auf, wenn die Aussprossung mit funktional falschen Zentren eine Verbindung aufbaut. Schneider (1973) führte im Tierexperiment diesen Nachweis für das visuelle System. Er wies nach, daß teilweise sogar die contralaterale Region durch die neuen Verbindungen innerviert wurde. Von Funktionswiederherstellung im eigentlichen Sinne konnte in diesem Fall jedoch nicht mehr gesprochen werden.

Die Denervierungsüberempfindlichkeit stellt keine eigentliche Regeneration dar, sie ist aber hinsichtlich der Wiederherstellung von großer Bedeutung. Sie ist an Rezeptoren von Neuronen zu beobachten, die aufgrund des Ausfalls einiger Synapsen auf einem geringeren Niveau innerviert werden, die geringere Innervation aber nach einiger Zeit durch eine erhöhte Empfindlichkeit ausgleichen. Hierfür müssen noch einige der rezeptiven Neuronen intakt geblieben sein. Zuerst wurde diese Wirkung von Canon und Rosenblueth (1949) beschrieben, die afferente Fasern, die zu einem Muskel führten, durchtrennten. In der Folge reagierte dieser Muskel auf die Gabe der normalen Menge des Neurotransmitters mit einer übersteigerten Reaktion. Der Übertrag auf das ZNS folgt jedoch lediglich einer Plausibilitätsheuristik und ist als solche nicht gesichert.

Ähnlich zu betrachten ist die Erholung durch Disinhibition, die von Wall und Egger (1971) beschrieben wurde. Sie beobachteten bei deafferentierten Ratten, daß sich während einer Stimulation der Hinterbeine in denjenigen thalamischen und cortikalen Gebieten, die die Hinterbeine repräsentierten, keine evozierten Potentiale mehr ableiten ließen, während die entsprechenden Gebiete der Vorderbeine bei Stimulation normal reagierten. Dieser Zustand hielt drei Tage an. Dann begann das Gebiet, das die Hinterbeine repräsentierte, zunehmend auch auf die Stimulation der Vorderbeine zu reagieren, bis schließlich das Reaktionsausmaß nicht mehr von dem Areal der Vorderbeine zu unterscheiden war. Da eine kollaterale Aussprossung unwahrscheinlich schien, wurde vermutet, daß es schon vormals in diesem Gebiet Synapsen gegeben hatte, die jedoch funktional ohne Bedeutung waren. Sie wurden solange gehemmt, wie die anderen funktionalen Verbindungen bestanden. Nach deren Ausfall kam es zu einer Disinhibierung. Insgesamt scheinen sich verschiedene Teile des Gehirns hinsichtlich ihrer Innervation zu überlappen, so daß Spezifität weitgehend durch 
Inhibition erzeugt wird. Fällt diese Hemmung weg, besteht die Möglichkeit der Funktionsübernahme durch die nun disinhibierten Areale.

Unter Vikariation wird die Übernahme von verlorengegangenen Funktionen durch benachbarte Cortexareale nach einer umschriebenen Läsion verstanden, wie es Munk mit der gleichnamigen Hypothese 1877 erstmalig postulierte. Fraglich ist, ob aber tatsächlich direkt benachbarte Gebiete in diesen Prozeß involviert werden. Wahrscheinlicher scheint, daß funktionell verwandte Gebiete, die aber lokalisatorisch nicht beieinander liegen müssen, die verlorengegangenen Funktionen übernehmen. Für den primärmotorischen Cortex stehen beispielsweise vor allem im supplementärmotorischen (SMA) und dem prämotorischen Cortex (PMC) lokalisierte Areale zur Verfügung, die eine Funktionsübernahme teilweise erlauben (Freund \& Hummelsheim, 1985). Dabei müssen diese neuen Areale die Funktion nicht in genau der Art ausüben, in der es das geschädigte Gebiet getan hatte. Es genügt, wenn die gleiche 'Rolle' übernommen wird. Ein Subsystem kann durch unterschiedlich beschaffene Reorganisation eine neue Aufgabe übernehmen, da nicht die Strategie, wie die Funktion kontrolliert wird, identisch sein muß, sondern nur der Umstand gesichert, daß funktional wieder die gleiche Kontrolle ausgeübt wird. Da im ZNS keine Gebiete bekannt sind, die ohne Aufgabe sind, wird durch die These der Vikariation die Frage aufgeworfen, ob die Kontrolle für die ursprünglich in diesem Gebiet repräsentierten Funktionen mit der gleichen Effizienz geschieht oder ob Einbußen in der ursprünglichen Funktion zu erwarten sind. Milner (1974) untersuchte Kinder hinsichtlich ihrer Sprachentwicklung und fand bei frühen Schädigungen der linken Hemisphäre eine Übernahme der Sprachfunktion durch die rechte bei jedoch gleichzeitig insgesamt herabgesetztem Intelligenzniveau. Milner (1974) interpretierte diese Beobachtung als eine Überlastung nach einer funktionellen Substitution.

Einem grundsätzlich anderen Gedankengang folgt die Theorie der Redundanz, die eng mit der Theorie der Äquipotentialität (Lashley, 1929) verbunden ist. Diese Theorie besagt, daß bereits ein Teil eines neuralen Systems die Funktion ausüben kann, für die normalerweise das gesamte System notwendig ist. Auf das Gehirn bezogen bedeutet dies, daß innerhalb einer Hirnregion das gesamte Gebiet an der Funktion beteiligt ist und, fällt ein umschriebener Teil aus, den speziellen Funktionsteil übernehmen kann. Die Grundlagen für diese Annahmen basieren jedoch auf Versuchen mit Ratten, so daß die Übertragung auf Menschen, deren ZNS-Strukturen sehr viel differenzierter und spezialisierter sind, nicht ohne weiteres zulässig ist.

Die Theorie der 'Multiplen Kontrolle' (Rosner, 1970) besagt, daß bestimmte Funktionen durch verschiedene Gehirnbereiche gleichzeitig kontrolliert werden. So läßt sich erklären, 
daß nach der Schädigung eines bestimmten, für spezifische Aufgaben zuständigen Gebiets kein Funktionsausfall stattfindet. Mit dieser Theorie werden nicht nur Aussagen über die Funktionswiederherstellung gemacht, sondern auch erklärt, warum es bei mancher Schädigung erst gar nicht zu einem Funktionsverlust kommt. Nach heutigem Verständnis scheint es aber unwahrscheinlich, daß es tatsächlich Zentren mit dem gleichen funktionellen Status gibt, da in Tierversuchen unterschiedliche Reaktionen hervorgerufen werden konnten, je nachdem, ob Hirnstrukturen simultan oder sequentiell geschädigt wurden. Nach der einfachen Theorie der multiplen Kontrolle hätten beide Versuchsvariationen zu einem gleichen Ergebnis führen müssen (Sturm, 1989). Daß für die gleiche Funktion jedoch verschiedene Kontrollzentren existieren, steht außer Zweifel. Ungeklärt ist, in welcher Art diese zusammenwirken.

Diaschisis, die schon bei den Schädigungsmechanismen erwähnt (siehe Kap. 2.1.2, S. 14) und als der ,... Funktionsverlust bzw. die Funktionsänderung (z. B. durch den weggefallenen exzitatorischen oder inhibitorischen Input) in umschriebenen Regionen des Hirns, der sich in der Folge einer anderswo lokalisierten, jedoch anatomisch verbundenen Hirnläsion einstellt“ (Hummelsheim, 1996 a, S. 136), definiert wurde, hat auch bei der Funktionswiederherstellung einen Einfluß. Nachgewiesen wurde dieser Effekt, nachdem er schon Anfang des Jahrhunderts beschrieben wurde (Monakow, 1905), beispielsweise in den contralateral gelegenen Gebieten nach einem A.-cerebri-media-Infarkt (Dobkin, Levine, Lagreze, Dulli, Nickles \& Rowe, 1989) oder im Cerebellum (Pappata, Mazoyer, Dinh, Cambon, Levasseur \& Baron, 1990). Durch die veränderte Innervation kommt es zu einem veränderten Metabolismus, der das entsprechende Gebiet nachhaltig schädigen kann (Feeny \& Baron, 1986). Normalisiert sich der Stoffwechsel durch pharmako-therapeutische Maßnahmen, ist von außen eine Wiederherstellung der durch diese Areale kontrollierten Funktionen zu beobachten. Bei dieser Funktionswiederherstellung handelt es sich also ,... nicht um einen aktiven Restitutionsprozeß, sondern um die Wiederkehr einer durch Diaschisis maskierten Funktion“ (Pöppel \& Steinbüchel, 1992, S. 28).

Ein für die motorische Wiederherstellung sehr wichtiger Ansatz ist die Long-TermPotenzierung (LTP), die - nach der allgemeinen Annahme, daß ein neuronaler Schaltkreis mit häufiger Benutzung immer besser funktioniert - von Bliss und Lomo (1973) für neuronale Systeme, insbesondere der Verbindung des Hippocampus mit Lernleistungen, erstmals genauer beschrieben wurde. Die Kernannahme der Long-Term-Potenzierung beschreibt eine ,,... länger anhaltende Zunahme exzitatorischer postsynaptischer Potentiale (EPSP) im Anschluß an eine kurze tetanische Stimulation der entsprechenden Afferenzen“ 
(Hummelsheim, 1996 a, S. 136). Den experimentellen Nachweis für die LTP im motorischen Bereich führten Asanuma und Keller (1991), die nach einer tetanischen Stimulation des somatosensiblen Cortex noch zwei Stunden danach eine größere Amplitude des EPSP in den motocortikalen Zellen ableiten konnten. Auf das motorische Lernen bezogen impliziert dies eine Aufforderung zum repetitiven Üben. Anfangs wird eine Bewegung gewöhnlich langsam ausgeführt und ist stark von sensorischen Rückmeldungen abhängig; im Rahmen der zunehmenden Automatisierung der Bewegung sinkt diese Abhängigkeit (vgl. auch die Annahmen der deduktiven Ansätze in 'Die Fähigkeit der motorischen Systeme', Kap. 2.1.3.2, S. 24 ff.). Auf der neurophysiologischen Ebene bedeutet dies, daß die permanente sensible Rückmeldung eine umschriebene motocortikale Neuronenpopulation exzitatorisch beeinflußt und somit die Innervation der an der Bewegung beteiligten Muskelgruppe fazilitiert. Repetitives Durchführen von Bewegungen induziert eine LTP, die Aktivierung der entsprechenden Neuronensets wird so auch langfristig begünstigt.

\subsection{Motorikspezifische Merkmale der zentralnervösen Funktionswiederhe- stellung}

\subsection{1 Physiotherapeutische Ansätze}

Verschiedene krankengymnastische Ansätze werden erläutert. Vielfach fehlt eine wissenschaftliche Überprüfung der theorieimmanenten Annahmen. Aufmerksamkeit stellt einen wichtigen zentralen Fazilitationsmechanismus dar. Der Mngel an theoretischem Basiswissen erklärt sich aus der Geschichte der Physiothenpie.

Nahezu alle Patienten, die im Zusammenhang mit einer neurologischen Erkrankung eine motorische Behinderung entwickelt haben, erhalten krankengymnastische und spätestens während der medizinischen Rehabilitation ergotherapeutische Übungsbehandlungen. Den quantitativ größten Anteil bilden dabei Patienten mit zentralen Hemiparesen, insbesondere auf dem Boden zerebraler Ischämien oder Blutungen. Anders als bei chirurgischen, pharmakologischen oder radiologischen Therapiemaßnahmen besteht für die verschiedenen krankengymnastischen Behandlungsmethoden kein nachvollziehbarer und geprüfter Indikationskatalog; ebensowenig sind Nebenwirkungen und Risiken der verschiedenen Verfahren hinlänglich bekannt. Die Berücksichtigung unerwünschter Effekte ist jedoch um so wichtiger, als die bislang - spärlich vorliegenden Studien zum Vergleich der Wirksamkeit verschiedener krankengymnastischer Übungsverfahren keine Überlegenheit irgendeines Verfahrens nachweisen konnten. (Hummelsheim, 1996 b, S. 143) 
Diesem eher kritischen Hinweis folgt die Darstellung der wichtigsten physiotherapeutischen Ansätze. Sie geben auch einen ersten Eindruck über - dort implizierte - Annahmen zum motorischen Lernen im Rahmen der Funktionswiederherstellung. Gemeinsam ist diesen Ansätzen, daß sie die funktionelle und strukturelle Plastizität des Nervensystems benutzen, um die motorische Leistungsfähigkeit des Patienten zu verbessern. Ziel ist die Reduzierung eines spastisch erhöhten Muskeltonus, die Erhaltung oder Verbesserung der aktiven und passiven Beweglichkeit, die Optimierung der Haltungs- und Gangstabilität, die Anbahnung von Bewegungen, die Förderung von Kraft und Ausdauer sowie die Verbesserung feinmotorischer Leistungen (vgl. Hummelsheim, 1996 b). Aufgeführt werden jedoch nur diejenigen Richtungen aus dem Verbund der Therapieschulen, die tatsächlich zentralnervöse Prozesse in den Mittelpunkt ihrer Funktionsmodelle stellen. Inhibition und Fazilitation, in der Krankengymnastik wichtige Behandlungsprinzipien, werden im Rahmen dieser Darstellung als Teil einer eher peripheren Beeinflussung vernachlässigt, die vor allem an dem Alpha-Motoneuron als dem ,... räumlichen und zeitlichen Summationspunkt peripherafferenter, deszendierender und interneuronaler Einflüsse“ (Hummelsheim, 1996 b, S. 144) ansetzen.

Die Brunnstrom-Methode (Brunnstrom, 1970) nutzt zur zentralen Fazilitation die intraund interhemisphärischen Verbindungen sowie bilaterale neuronale Schaltkreise. Die Patienten werden aufgefordert, die gelähmte Extremität zu bewegen, während sie gleichzeitig kontralateral die entsprechenden Muskeln gegen einen mechanischen Widerstand innervieren oder aber ipsilateral proximale Bewegungen initiieren. Durch Irradiationseffekte werden so zumeist grobe synergistische Masseneffekte ausgelöst. Durch die maximale Innervation der zu den plegischen Muskelgruppen contralateralen wird ein höheres Erregbarkeitsniveau in den Alpha-Motoneuronen der betroffenen Muskeln erreicht. Für die Fazilitation der Feinmotorik greift die Theorie jedoch auf periphere Techniken zurück, die Applikation von lokalen Haut- oder Muskelreizen wird empfohlen. Von zentraler Bedeutung ist auch die Betonung der Aufmerksamkeit; der Patient wird aufgefordert, sich ganz auf die zu bewegende Muskelgruppe zu konzentrieren, um so einen zentralen Fazilitationseffekt auszulösen.

Die von Rezeptoren in den Vestibularorganen und in der Halsmuskulatur vermittelten Tonusverschiebungen und dadurch evozierten Gleichgewichts- und Komplexbewegungen stehen im Mittelpunkt und am Anfang der Methode von Bobath (1965). Dieser Ansatz beinhaltet jedoch eine in bezug auf die Behandlung Erwachsener theoretisch nicht begründete Übertragung von Beobachtungen bei zentral-paretischen Kindern auf hemiparetische Erwachsene. Dies führte im Verlauf der nachfolgenden Jahre zu Schwierigkeiten, da sich die 
postulierten Prinzipien bei diesen nur schwer nachweisen ließen (für eine aktuelle Untersuchung siehe Hummelsheim \& Hoppe, 1994). Eine Zeitlang wurden zentrale Fazilitationstechniken aus diesem Grund überhaupt nicht mehr angewendet. Eine weitere Begründung war das Auftreten von assoziierten Bewegungen bei dem Versuch einer willkürlichen Bewegung als eine der zentralen Fazilitationstechniken sowie eine spastizität-begünstigende Tonussteigerung als allgemeine Folge zentraler Fazilitationen. Mittlerweile stellt aber das bewußte Bewegen auch in diesem Konzept einen wichtigen zentralen Fazilitationsmechanismus dar: „Die Behandlung hilft dem Patienten durch Muster, die Spastizität zu hemmen, Kontrolle über die ungehemmte tonische Reflexaktivität zu entwickeln und zu verbessern. Mit dieser Hemmung werden seine Bewegungen in normalere Funktionsmuster geführt“ (Bobath, 1993, S. 6).

Für die Therapie nach Vojta (1976), die sich in der Hauptsache auf die von ihm postulierten genetisch verankerten Bewegungsprogramme stützt, sind drei Merkmale unerläßlich: Eine ausgewogene, automatisch gesteuerte Körperlage, die alle Körperabschnitte systemorientiert und geordnet einbezieht, charakteristische Aufrichtmechanismen im Bereich des Kopfes, Rumpfes und der Extremitäten und die phasische Muskelarbeit, die abgestimmt ist auf die Schwerpunktverlagerung über verschiedenen Punkten (Pape, 1997). In bestimmten Ausgangsstellungen werden unterschiedliche Koordinationskomplexe ausgelöst, der therapeutische Zugriff auf das ZNS erfolgt dabei reflektorisch. Während der Rehabilitation soll der Patient lernen, diese Auslösemechanismen einzusetzen, um seine motorischen Funktionen - jederzeit reproduzierbar - selbst aktivieren zu können.

Eine sensorisch-fazilitatorische Idee beinhaltet der Ansatz Affolters (1987). Demnach ist der hirngeschädigte Patient in seiner Fähigkeit beeinträchtigt, diejenigen Informationen aus der Umgebung wahrzunehmen, die Bewegungs- oder Handlungsrelevanz besitzen (vgl. auch die soziobiologischen Annahmen zur Verbindung von Wahrnehmung und Handlung, Kap. 2.2.2.1, S. 58 ff.). Sie explorieren unzureichend und verbleiben in ihrer Wahrnehmung in einem kleinen, selbst geschaffenen Bezugsrahmen. Die therapeutische Übungsbehandlung versucht, die Kapazität der Informationsaufnahme zu erweitern. So steht vor allem das 'Spüren' im Mittelpunkt. Bewegungen werden nicht per se geübt, sondern in Abhängigkeit von Situationen und Dingen mit jeweils ausreichend starkem Aufforderungscharakter trainiert.

Die Organisation des Systems und nicht die periphere Ebene wird von der kognitivtherapeutischen Annäherung Perfettis (1981) als zentral erachtet, wobei ein Schwerpunkt auch hier die Sensorik darstellt. Um Bewegung ausführen zu können, braucht das Gehirn Vorinformationen von dem Körper und von der Umgebung: „Bewegungen und Wahrneh- 
mung bedingen sich also gegenseitig: Durch Bewegung kommt man zu Informationen, und die Informationen ermöglichen das Entstehen einer Bewegung“ (Oberleit, 1996, S. 536). Zusätzlich evoziert sensorische Stimulation eine Differenzierung der inneren Repräsentation des eigenen Körpers. Ein anderer Theoriekern stellt die Betonung der Aufmerksamkeit dar, ohne die, nach therapieimmanenten Annahmen, Lernen nur bedingt stattfinden kann. Nach Perfetti (1981) wird die in der neurologischen Rehabilitation zentrale Spastizität durch vier Komponenten verursacht. Ihr liegen ein übersteigerter Stretchreflex, eine abnorme Irradiation, synergistische Schemata und Defizite in der Muskelrekrutierung zugrunde. Bei der Untersuchung wird also nicht mehr das Ausmaß der Spastizität geprüft, sondern „... wo und wie stark diese vier pathologischen Elemente vorhanden sind und vor allem auch, inwieweit der Patient schon über die kognitive Ebene auf diese Elemente einwirken kann“ (Oberleit, 1996, S. 539).

Weitere Ansätze (PNF, Rood etc.) beziehen sich auf hauptsächlich periphere Fazilitationstechniken (thermische oder mechanische Reize, Schmerzreize, rasche Muskeldehnung etc.) und werden von daher in diesem Kontext nicht weiter ausgeführt.

Wie in der Darstellung deutlich wird, bleibt ein expliziter Hinweis auf zugrundeliegende Lernmechanismen weitestgehend aus. Zentrale Fazilitation wie Aufmerksamkeitszuwendung oder sensorischer Input werden postuliert und in die Therapieimplikationen eingeflochten. Dies geschieht jedoch ohne konkrete Annahmen, wie diese in das motorische Lernen eingebunden sind, wie sie also über die krankengymnastische Behandlung hinaus eine Wirkung haben können. Eine mögliche Synthese wäre, daß die physiotherapeutischen Maßnahmen erste, rudimentäre Bewegungen vermitteln, während das Lernen von komplexeren Bewegungen anschließend einsetzt. Sie entspricht jedoch lediglich der Realität der Frührehabilitation. Im Anschluß an diese werden krankengymnastische Maßnahmen durchaus angewandt, um komplexere Bewegungsabläufe zu vermitteln.

Ein Grund für diesen Mangel an zugrundeliegenden Theorien weist Hüter-Becker (1997) auf, die auf der Basis einer Arbeit der Kommission 'Strukturanalyse' des Gesamtvorstandes des Deutschen Verbandes für Physiotherapie bzw. des Zentralverbandes der Physiotherapeuten/Krankengymnasten die grundlegende Struktur der Krankengymnastik vor dem historischen und aktuellen Hintergrund aufdeckt: „Die Physiotherapie imponiert mit einem fast unüberschaubaren Angebot von Untersuchungs- und Behandlungstechniken; eine durchgängige Struktur mit verbindenden Elementen fehlt, zumindest wird sie nicht auf den ersten Blick deutlich“ (Hüter-Becker, 1997, S. 566). Neben der fehlenden 'Einheit in der Vielfalt' (ebd.) verweist eine vielleicht unbeabsichtigte Begriffswahl auf tiefere Hinter- 
gründe: Stehen 'Techniken' im Vordergrund, hat die grundlagenwissenschaftliche Theoriebildung zumeist nur noch wenig Raum, wenngleich die Definition der Technik im engeren Sinne durchaus auf die „.. Gesamtheit aller Mittel, die Natur auf Grund der Kenntnis $u$. Anwendung ihrer Gesetze dem Menschen nutzbar zu machen“ (Wahrig, 1986, S. 1267; kursiv hinzugefügt) abzielt und somit die Theoriebildung der Entwicklung von Behandlungstechniken zeitlich vorgeschaltet sieht. Des weiteren besteht die Krankengymnastik die aufgrund zeitgemäßer Ansätze eher als Bewegungstherapie bezeichnet werden sollte, da nicht mehr die Kranken allein im Vordergrund stehen (Hüter-Becker, 1997) - aus den vier Schwerpunkten der Funktionen des Bewegungssystems, der Funktionen innerer Organe, der Bewegungsentwicklung bzw. -kontrolle sowie des Verhaltens und Erlebens. Innerhalb derer kann nur der Bereich der Bewegungsentwicklung zum Bewegungslernen und damit potentiell zu zugrundeliegenden Prozessen Aussagen implizieren (Hüter-Becker, 1997, S. 567). Der einzige substantielle Schritt hin zur Grundlagenwissenschaft, der sich aus dem Wink des häufig gebrauchten Ausdrucks '... auf neurophysiologischer Grundlage' ableiten läßt, wird mit dem Hinweis nivelliert, ,... daß der Begriff 'Behandlung auf neurophysiologischer Grundlage' oder 'neurophysiologische Techniken' nicht nur auf eine begrenzte Auswahl angewendet werden kann, weil Bewegung, sei sie passiv geführt oder aktiv ausgeführt, immer auf neurophysiologischer Grundlage geschieht“ (Hüter-Becker, 1997, S. 568). Wenig nützlich und von ähnlich geringem heuristischen Wert ist abschließend auch der Hinweis, daß die gemeinsame Grundlage aller Behandlungstechniken „,... das Nervensystem und andere körpereigene Informationsnetze“ (Hüter-Becker, 1997, S. 568) sind. Einen weiteren Problembereich weist Hummelsheim (1996 b) auf:

Alle genannten [Bobath, Kabat, Knott, Vojta, Rood und Brunnstrom] krankengymnastischen Übungsverfahren nehmen für sich in Anspruch, ,,auf neurophysiologischer Grundlage“ zu arbeiten. Obwohl dies wahrscheinlich prinzipiell zutrifft, bemühen sich die verschiedenen krankengymnastischen Schulen um verschiedene Zielsymptome der gestörten Motorik. Die jeweils anderen Aspekte kommen oftmals zu kurz, nicht zuletzt als Folge einer z. T. dogmatischen Ausgrenzung der Inhalte anderer Schulen. (Hummelsheim, 1996 b, S. 143)

In der einschlägigen Fachliteratur imponiert die Aprilausgabe 1997 des offiziellen Organs des Deutschen Verbandes für Physiotherapie und des Zentralverbandes der Physiotherapeuten/Krankengymnasten (ZVK) e. V. 'Krankengymnastik', dessen Themenschwerpunkt das Bewegungslernen ist. In den einzelnen Aufsätzen finden sich aber wiederum nur me- 
thodisch-didaktische Hinweise (,,.. welche Möglichkeiten die Funktionelle Bewegungslehre hat, um realistische Behandlungsziele zu ermitteln ... es wird betont, daß die natürliche Bewegung mehr ist als Beweglichkeit, Kraft und Geschicklichkeit. Sie ist untrennbarer Teil der Persönlichkeit des Menschen“ (Bronner 1997, S. 578)), epistemologisch wenig wertvolle Aussagen (,,... als Voraussetzung für die effektive Vermittlung therapeutischer Übungen wird die Kommunikation zwischen Patient und Therapeut über auditive, visuelle und kinästhetische Sinnenkanäle [sic] herausgestellt" (Volk, 1997, S. 598)) oder detaillierte Beschreibungen von Techniken (,,.. am Beispiel des Standbeintrainings bei spastischer Hemiparese werden die entscheidenden Aspekte der Funktionellen Bewegungslehre von Susanne Klein-Vogelbach für die Gangschulung bei zentralmotorischen Störungen dargestellt. Schlüsselpunkte des Standbeintrainings sind die Fersenbelastung und der Abrollvorgang des betroffenen Fußes“ (Künzle, 1997, S. 588)). In dem - neben zwei Aufsätzen der USAmerikanischen Forscherin Dr. Darcy Umphred (1997 a, b) - einzigen grundlagenbezogenen Artikel „Bewegungslernen - Auf dem Weg zu mehr Wissen und Verständnis“ (Schewe, 1997) findet sich schon in der Einleitung eine Bankrotterklärung des Versuchs einer wissenschaftlichen (Physio-)Theoriebildung: „Es hat sich in den Wissensbereichen, die ich als 'Menschenwissenschaften' zu bezeichnen pflege, gezeigt, daß nicht die Theoretiker das Wissen voranbringen, sondern diejenigen, die sich in ihrer täglichen Arbeit mit den praktischen Problemen auseinandersetzen müssen. Das hat damit zu tun, daß der Mensch oder das System Mensch so komplex ist, daß wir es, und seien wir noch so klug, mit unserem begrenzten Verstand nicht vollständig erfassen können“(Schewe, 1997, S. 624).

Bütefisch, Hummelsheim, Denzler und Mauritz (1995) urteilten, nachdem sie in einer Untersuchung zur Erholung der Handfunktionen herausfanden, daß die einfache Übungswiederholung ('repetition') den klassischen Therapieansätzen überlegen war:

... although the neurophysiological background of all these concepts is poorly understood because of lack of basic research in this field .... The physiotherapeutic method according to Bobath emphasizes the reduction of the enhanced muscle tone before active movements are facilitated by means of cutaneous and proprioceptive stimuli applied to the region of the target muscles. The Brunnstrom or PNF concepts, however, use maximal innervation of intact or less paretic muscle groups to produce irradiation effects in more severely paretic synergistic muscle groups. .... Surprisingly, none of the commonly used traditional physiotherapeutic strategies emphasized specifically the voluntary activation of the distal arm and hand musculature. Innvervation 
is rather stimulated by cutaneous and proprioceptive (Bobath) as well as central (Brunnstrom, PNF) facilitation techniques. (Bütefisch, Hummelsheim, Denzler \& Mauritz, 1995, S.1)

\subsection{2 Motorisches Lernen nach ZNS-Schädigungen}

Neuronale Korrelate für das motorische Lernen scheinen erstmals in der Wissenschaftsgeschichte in die Reichweite der Erkenntnisgewinnung zu gelangen. Operante und klassische Konditionierung sind beim Lernen noch immer wichtige Grundlagen, deren Theorieelemente in Verbindung mit den zentralen Mustergeneratoren vermutlich das Substrat des mtorischen Lernens darstellen.

Die Frage, wie Programme neu erworben werden, ist nur in wenigen motorischen Lerntheorien explizit behandelt worden. Viele Bewegungsforscher vertreten die Auffassung, daß dieser Art des Lernens keine allzu große Bedeutung zukommt. (Roth, 1987, S. 254)

Im Gegensatz zu der im Zitat ausgedrückten Tendenz sollen hier die neuronalen Grundlagen des motorischen Lernens behandelt werden, die Ebene der strukturellen Betrachtungsweise wurde bereits weiter oben angerissen (Kap. 2.1.3.1, S. 29 ff.). In den meisten Fällen handelt es sich bei den Theorien zum motorischen Lernen um Modelle der Bewegungskoordination, die um den Veränderungsaspekt erweitert wurden. Diese Veränderung findet, in Abhängigkeit von den zugrundeliegenden Modellen, sowohl auf zentrale als auch auf periphere Steuerungsmechanismen bezogen statt.

Die neuronalen Grundlagen des motorischen Lernens sind nicht vollständig bekannt, selbst die zu beschreibenden Modell-Netzwerke sind nur Analogien und nicht Korrelate neuronaler Verschaltungen (Menzel, 1996). Doch scheint es, als seien in den letzten Jahren zum ersten Mal die Voraussetzungen geschaffen worden, daß die Psychologie von den interdisziplinären Biowissenschaften eingeholt wird. Jahrzehntelang war es in der Psychologie unbestritten, daß ein Warten auf physiologische Korrelate psychologischer Phänomene in der psychologischen Erkenntnisgewinnung eine Verzögerung des Fortschritts in Kauf nehmen würde. Auch wenn sich dieser Tatbestand noch nicht grundsätzlich verändert hat, wird doch der Abstand zwischen diesen Wissenschaftszweigen immer geringer. Das Gehirn verliert sowohl ein Stück der es immer noch umgebenden Mystik und wird durch diesen Fortschritt dem ZNS anderer Vertebraten und auch vieler Invertebraten immer ähnlicher. 
Zum ersten Mal in der Geschichte der Biologie wird sichtbar, wie durch äußere Reize, die über die Sinnesorgane aufgenommen werden, Gene geschaltet werden, Strukturen der neuronalen Netze verändert werden und Verhalten auch in seinen komplexen Anpassungsweisen erzeugt wird. Die Neurowissenschaft ist auf dem Weg, den Informationsfluß und seine Verarbeitung auch in hochentwickelten Nervensystemen zu erkennen und auf seine Elementarereignisse zurückzuführen. Damit gerät unser eigener Denk- und Wissensapparat, unser Gehirn, in die Reichweite einer naturwissenschaftlichen Disziplin. (Dudel, Menzel \& Schmidt, 1996, S. V)

Im Mittelpunkt des Theoriekomplexes der neuronalen Grundlagen des motorischen Lernens steht der sogenannte zentrale Mustergenerator (ZMG; vgl. Rathmayer, 1996). Dieser besteht bei Invertebraten aus prämotorischen Neuronenensembles mit der intrinsischen Fähigkeit zu rhythmischer Entladungsaktivität. Die erzeugten Muster werden an die nachgeschalteten Motoneuronen weitergegeben. So kommen die stereotypen, von Außenreizen weitgehend unabhängigen Bewegungsabläufe zustande, wie sie beim Flügelschlag von Insekten oder Schwimmbewegungen der Meeresschnecke zu beobachten sind. Nicht alle Bewegungen laufen aber derart hochautomatisiert ab, vielmehr basiert ein Großteil von Bewegungen auf einer Interaktion des ZMG mit sensorischen Rückmeldungen. Im Rahmen dieser Interaktion existieren alle Ausprägungen der verschiedenen Kombinationen, vom Schwimmuster der Blutegel, das überwiegend von zentralen Mustergeneratoren gesteuert wird, über den Schwanzschlag eines Krebses auf der Flucht, der durch Kommandoneuronen initiiert wird, dann aber autonom unter der Steuerung des ZMG weiterläuft, der Meeresschnecke Tritonia, die auf der Flucht alternierend den Körper nach dorsal und ventral krümmt, was ihr als Schwimmbewegung dient und damit ein Beispiel für dynamischinteragierende, multifunktionelle ZMG darstellt, bis zur Beinbewegung der Heuschrecke, bei der die afferenten Meldungen der Extero- und Propriorezeptoren von entscheidender Bewegungswichtigkeit sind (Rathmayer, 1996).

Lernen ist der Vorgang, durch den Informationen über die Umwelt gewonnen werden. Es hat stattgefunden, wenn ein systematischer Zusammenhang zwischen zurückliegender Erfahrung und aktuellem Verhalten festgestellt werden kann, wobei Erfahrung die assoziative Verbindung zweier Ereignisse meint, die so in einen Ursache-WirkungsZusammenhang gebracht werden: „Durch Assoziation erhalten Ereignisse eine Bedeutung, und dieser Informationsgewinn steht auch nach langem Zeitintervall als Gedächtnis zu Verhaltenssteuerung zur Verfügung“ (Menzel, 1996, S. 500). Zwei Lernformen müssen dabei 
grundsätzlich unterschieden werden, da nur bei der ersten nachfolgenden Art des Lernens das limbische System beteiligt ist: Explizites (deklaratives) Lernen bezieht sich auf bewußt werdende Lerninhalte hinsichtlich anderer Menschen, Orte und Dinge, die sehr schnell in bildhafter Weise gespeichert werden, während implizites Lernen (prozedurales) bei motorischen Vorgängen und einfachen Wahrnehmungsleistungen, die weitestgehend automatisch und nach den Regeln der assoziativen Konditionierung gespeichert werden, vorkommt (Anderson, 1989). Daß explizites Lernen auf die Beteiligung des Hippocampus angewiesen ist, liegt nach heutigem Stand des Wissens an der für den Lernakt unerläßlichen Einbindung des neu zu Lernenden in bereits bestehende Wissensstrukturen, an der Teile des limbischen Systems beteiligt sind.

Motorisches Lernen, d. h. die Aneignung und Entwicklung motorischer Fertigkeiten und Verhaltensweisen ist ein komplexer, lebensbegleitender Prozeß, der sich auf qualitativ unterschiedlichen Ebenen vollzieht. Der Begriff motorisches Lernen oder Bewegungslernen faßt eine Vielzahl von parallelen Teilprozessen der Adaptation und Ökonomisierung in den unterschiedlichen Systemen und Teilen des ZNS bei gleichzeitiger konditioneller Vervollkommnung zusammen. (Schober, 1992, S. 59)

Die Suche nach den neuronalen Elementen für die operante Konditionierung, deren Theorieelemente in Verbindung mit den ZMG vermutlich das Substrat des motorischen Lernens darstellen, geht von drei basalen Phänomenen aus: Das Verhalten kann in minimale Komponenten - sogenannte 'Verhaltensatome' (Skinner, 1953) - aufgeteilt werden, es hat eine stark spontane Komponente und assoziatives Lernen tritt nur auf, wenn eine enge zeitliche Kontiguität zwischen einem Verhaltenselement und seiner Verstärkung besteht (Menzel, 1996).

Die Basis für das klassische Konditionieren, das der operanten vorgeschaltet ist, bilden zwei Tatbestände: Die aktivitätsabhängige Neuromodulation und die prä- bzw. postsynaptische Erregungskoinzidenz. Auf der neuronalen Ebene bildet die Modulation der Erregungseigenschaft einzelner Neurone, die zu multiplen Netzwerkeigenschaften von Zellensembles führt, die Grundlage für einfache Formen von neuronaler Plastizität. Den neuronalen Vorgang beschreibt Schober (1992), der davon ausgeht, daß sich ,... nach der Aktivierung der Synapse (CS) dort eine kurzzeitige lokale Erregung ausbildet, die durch die nachfolgende Erregung der Synapse (UCS) sowie durch die ausgelöste Depolarisation weiter verstärkt wird. Zugleich erfolgt durch die Zellaktivierung eine unspezifische Stimulation der RNS- und Proteinsynthese, möglicherweise auch durch den Einfluß 
motivationaler Eingänge. Emotionale Reaktionen, die durch aminerge Eingänge vermittelt werden, induzieren eine Steigerung der Glycoproteinsynthese. Diese Glycoproteine werden in den Dendriten transportiert. Ihr Einbau in die postsynaptische Membran der vorher kurzzeitig erregten Synapsen stabilisiert dauerhaft deren Konnektivitätsänderung und erreicht damit die bevorzugte Bahnung der Bewegungsabläufe und Reaktionen bei erneuter alleiniger Aktivierung der Synapse“ (Schober, 1992, S. 63)6. Dabei findet Lernen nicht nur in den höheren ZNS-Strukturen statt. „The role of the spinal cord is central to many motor tasks and it now seems clear that networks within the spinal cord are themselves modified in some learning processes“ (Hodgson, Roy, Leon, Dobkin \& Edgerton, 1994, S. 1492). Nach Versuchen mit Katzen wurden auch Patienten mit einer kompletten Querschnittslähmung trainiert, indem die Beine in einem Laufapparat in eine Laufbewegung versetzt wurden. „Generally, the EMG burst patterns were more consistent in the fifth compared with the first minute of a training session. Waveforms were also less variable from day to day with training than they were if training stopped for more than 3-5 days and then resumed“ (Hodgson, Roy, Leon, Dobkin \& Edgerton, 1994, S. 1496).

Bei Tieren setzt das Lernen ein, nachdem die zentralen Mustergeneratoren spontan Verhalten produzieren, das anschließend operant durch Erfolg und Mißerfolg positiv bzw. negativ verstärkt wird. Motorische Programme für komplexe Bewegungsabläufe entstehen so durch die Interaktion von ZMG und sensorischen Rückmeldungen. Ist ein Programm erfolgreich, stabilisiert es sich über Neuromodulatoren wie biogene Amine oder bestimmte Peptide, die ,... im Gegensatz zu konventionellen Neurotransmittern, deren Aktion in der Regel schnell und an lokal begrenzten Rezeptoren und deren Ionenkanälen an den postsynaptischen Zellen erfolgt, ... über ihre postsynaptischen Rezeptoren häufig langfristig intrazelluläre Signalwege in den Zielzellen“ (Rathmayer, 1996, S. 181) beeinflussen. Die relative Stabilität dieser Muster und auch ein Hinweis für die zentralnervöse Plastizität sieht Rothwell (1995) nach der Ableitung von Innervationsmustern bei Affen während bestimmter Bewegungen in dem Umstand begründet, daß unterschiedliche Neuronenpopulationen die gleiche Aufgabe übernehmen: „A picture emerges in which a smooth drawing movement is produced by different parts of the nervous system operating in parallel, with the relative contribution of each part varying at different stages of the task. Such an organiza-

\footnotetext{
${ }^{6}$ Die klassische Konditionierung hat, entgegen ursprünglicher Annahmen (Pavlov, 1967), auch kognitive Aspekte. Kamin (1968) wies nach, daß nicht nur die Kontiguität, die bislang als der einzig entscheidende Faktor angesehen wurde, sondern auch infomationelle Beziehungen zwischen den konditionierten und den unkonditionierten Stimuli für eine erfolgreiche Konditionierung notwendig sind.
} 
tion is typical of most biological systems and although complex, it has the advantage that rarely is one set of neurons absolutely necessary for any task. Their actions can usually be compensated for by other structures. This means that the system is not only flexible, but also highly resistant to damage“ (Rothwell, 1995, S. 102).

Beim Menschen scheinen ähnliche Mechanismen wie die bei Tieren beschriebenen wirksam zu werden. Setzt man Versuchspersonen eine Prismenbrille auf, die oben mit unten und rechts mit links vertauscht, finden sie sich nach einigen Tagen in der Welt zurecht, indem sie das durch die Brille umgekehrte Unten wieder als Oben sehen, die Wahrnehmung also wieder der erfahrbaren Welt entspricht (Gregory, 1973). Unerläßlich ist dabei aber sowohl die Bewegung in als auch die Auseinandersetzung mit der anfänglich verdrehten Welt. Ein hierbei lokalisierbares Zentrum für das motorische Lernen scheint das Cerebellum zu sein, bei dessen Ausfall die Fähigkeit zur Anpassung an neue motorische Aufgaben eingeschränkt ist (Blickhan, 1996). Vor diesem Hintergrund scheint es naheliegend, daß motorisches Übungslernen im aktiven Korrigieren fehlerbehafteter efferenter Programme besteht:

Es zeigt sich, daß der operante Lerneffekt in einem Vergleich zwischen dem generierten und dem ausgeführten motorischen Programm besteht. Die Sensorik meldet das ausgeführte Programm, und diese Meldung stellt das Verstärkersignal V dar. Bei zeitlicher Passung mit dem motorischen Programm wird das Entladungsmuster des ZMG stabilisiert. Abweichungen werden dadurch korrigiert, daß in den ständig fluktuierenden Erregungsmustern des ZMG jene stabilisiert werden, die den neuen Bedingungen am besten angepaßt sind. (Menzel, 1996, S. 510; kursiv im Original)

\subsection{3 Training der konditionellen Fähigkeiten}

Die Reizdosis muß der Leistungsfähigkeit des Patienten angepaßt werden. Die Steigerung der konditionellen Fähigkeiten ist auch eine direkte Folge der zentralnervösen Wiederherstellung. Das Training der konditionellen Fähigkeiten steht hüfig im Schatten des Trainings der koordinativen. Auf die Wichtigkeit der Kreislaufsituation wird hingewiesen. Eine Trianingsform ist das ideomotorische Training.

Die durch Training bewirkte Leistungssteigerung wird durch die Anpassungs- und Umstellungsfähigkeit des Organismus erreicht. Das Training muß in der Reizintensität derart gestaltet sein, daß die Reize weder unterschwellig noch zu stark sind. Im ersten Fall würde der Organismus nicht, im zweiten mit einer vorübergehenden oder dauerhaften Schädigung reagieren. Demzufolge muß sich die Reizdosis auch bei Hirnverletzten der Leistungsfähigkeit des Organismus anpassen. Das Training sollte je nach Schädigung und Alter mit einer 
Belastungsgrenze von zunächst 120, dann 130 bis 160 Schlägen/min erfolgen (Jochheim, 1955).

In der Ausdauerentwicklung sind zwei Methoden vorherrschend, denen viele spezifischere Formen zugeordnet werden können: Das extensive und das intensive Ausdauertraining. Das kennzeichnende Merkmal des extensiven Trainings ist die 'lohnende Pause', eine Trainingsunterbrechung von etwa 1 bis $1.5 \mathrm{~min}$, in der die Herzfrequenz bei gesunden Personen wieder auf etwa 120 bis 140 Schläge pro Minute absinken kann. Danach erfolgt der nächste Trainingsreiz. Beim intensiven Ausdauertraining bleibt die Belastung konstant. Unter gesundheitlichen Gesichtspunkten wird diese Methode zur Verbesserung der aeroben Kapazität empfohlen (Knebel, 1985). Das Krafttraining besteht aus allgemeinen und speziellen Kraftübungen, durchgeführt nach der Wiederholungs- oder Intervallmethode, bei der wiederum, unter Berücksichtigung der verschiedenen Kontraktionsformen (konzentrisch oder exzentrisch), mit gleichen oder wechselnden Belastungen gearbeitet werden kann. Die Trainingsformen ergeben sich so aus der Kombination der Komponenten Umfang, Intensität, Dichte und Dauer der Belastung bzw. der Pausen (Heipertz, 1985). Schnelligkeit per se läßt sich nur wenig trainieren. Lediglich in Sportarten, in denen Technikformen einen großen Anteil haben (z. B. Schwimmen), können Erfolge des Schnelligkeitstrainings nachgewiesen werden (Heipertz, 1985).

Doch genau wie der Verlust der koordinativen Fähigkeiten eine direkte Folge der zentralen Läsion ist, und auch Kraft- und Schnelligkeitsverlust einer zentralen Lähmung immanent sind (vgl. S. 37), wird auch die zentralnervöse Wiederherstellung von der Steigerung der konditionellen Leistungsfähigkeit begleitet. Anders als bei Gesunden handelt es sich also nicht nur um eine bewußte Störung der Homöostase mit dem Ziel der Anpassung und der damit verbundenen Leistungssteigerung. Der Verlust der konditionellen Fähigkeiten ist auf die zentrale Läsion zurückzuführen, der Wiedergewinn somit auf ihre Wiederherstellung. Das reine Konditionstraining nimmt in der neurologischen Rehabilitation nur eine beigeordnete Stellung ein. Krafttraining wird beispielsweise bei funktionell schwerer Betroffenen in der Ergotherapie in Form von Kneten durchgeführt, weniger schwer Geschädigte üben an Zugapparaten. Schnelligkeit wird nur implizit trainiert. Ausdauer wird über ein Fahrradergometertraining nach der Dauermethode systematisch vergrößert. Die einseitige Einschätzung jedoch, nach der ,der Schwerpunkt der Sporttherapie im bewegungstherapeutischen Bereich ... in der Koordinationsschulung [liegt, und die] ... Entwicklung konditioneller Fähigkeiten ... bei diesen Patienten weniger in Betracht“ (Merten \& Conradi, 1991, S. 814) kommt, kann nicht geteilt werden. Vielmehr gilt, wie eine Untersuchung zur Kreis- 
laufsituation nach Apoplexie zeigte, daß ,... künftig in der Schlaganfall-Nachsorge neben der Verbesserung der Koordination, der Linderung der Paresen und der medikamentösen Einstellung der überschießenden Blutdruckwerte eine noch stärkere Akzentuierung in Richtung auf eine Entlastung und Ökonomisierung des Kreislaufes wünschenswert erscheint“" (Bergholz, 1985, S. 1448 f.). Die Hypothese von Monga, Deforge, Williams und Wolfe (1988), ,... that cardiovascular response to exercise in stroke patients would be abnormal, with exercise-induced changes in HR [heart rate] and BP [blood pressure] of significantly lower magnitude than those of control subjects“ (Monga, Deforge, Williams \& Wolfe, 1988, S. 937), konnte in der nachfolgenden Untersuchung von den Autoren nicht bestätigt werden.

Ein zunehmend wichtiges Feld in der Rehabilitation motorisch Geschädigter stellt das ideomotorische Training dar, nach dessen Grundannahmen allein die intensive Vorstellung einer Bewegung die Tendenz zu ihrer Ausführung hervorruft (Baumann, 1993, S. 80). Nachdem anhand einiger Parameter wie Herzschlagfrequenz, Atemzugfrequenz oder Hautleitwert die positiven Effekte in gesunden Populationen nachgewiesen werden konnten, und die Verbesserung sensomotorischer Leistungen durch ideomotorisches Training in Verbindung mit der realen Ausführung der Bewegung als gesichert galt, wurde dieses Verfahren auch hilfreich in der Rehabilitation motorisch Behinderter eingesetzt (Beyer, 1991). 


\subsection{Belastungswahrnehmung}

Der Begriff 'Belastungswahrnehmung' wird eingeführt. Zwei Ebenen körperlicher Aktivität sind genauer zu betrachten, die Leistung und die Belastungswahrnehmung.

Unter Berücksichtigung verschiedener Ebenen sind für die körperliche Aktivität im Kontext dieser Betrachtung zwei Ebenen besonders von Bedeutung. Gemäß der 'Außensicht' äußert sich die Handlung des Aktiven als objektivierbare Leistung, aus der 'Innensicht' handelt es sich um die Wahrnehmung einer Belastung, welche verkürzt als Belastungswahrnehmung bezeichnet werden soll. Eine Position der Mitte nimmt die Belastung als solche ein, die über Messungen beispielsweise der Herzfrequenz, der Sauerstoffaufnahme oder des Laktatspiegels facettenartig objektivierbar ist und deren psychologische Entsprechung unter Einbezug und differenzierter Gewichtung aller Facetten wiederum die Belastungswahrnehmung ist.

\subsubsection{Leistung und Belastung}

Zusammenhänge zwischen Leistung und organismisch-objektivierbaren Variablen werden dargestellt. Als Teil der Bedstungstestung ist die kardio-zirkulatorische Funktionsprüfung von besonderer Bedeutung. PWC 170 (physical working apacity) drückt in Watt die fahrradergometrische Leistung aus, bei der eine Herzfrequenz von 170 Schlägen/min erreicht wird.

Die Messung der Leistung als „... ein wissenschaftlich begründetes Routineverfahren zur Untersuchung eines oder mehrerer organismischer Merkmale mit dem Ziel einer möglichst quantifizierten Aussage“ (Israel, 1979, S. 831) erfolgt bevorzugt mit Hilfe des Fahrradergometers, das eine Angabe der Leistung in Watt ermöglicht und damit eine Basis für in engen Grenzen - interindividuell vergleichbare Werte liefert. Ansonsten findet die Leistungsmessung in jedem Wettkampf statt, ausgedrückt in Zeiten und Strecken, oder durch Tests, in denen die Ausführung normiert ist und somit - jedoch in noch engeren Grenzen Vergleichbarkeit gewährleistet wird. Beurteilt man nur die absolute Leistung, gehört beispielsweise der weit verbreitete Cooper-Test ${ }^{7}$ in diese Kategorie, der mit der festen Zeit von zwölf Minuten auf der 400-Meter-Bahn durchgeführt wird und aus dem, da er eng mit der

\footnotetext{
${ }^{7}$ Der Cooper-Test, 1968 in der US-Luftwaffe entwickelt (vgl. Bös \& Multerer, 1990), ist ein Lauftest, der nach vielfältigen Versuchen letztendlich mit dem Zeitraum von zwölf Minuten veröffentlicht wurde. Die Aufgabe innerhalb des Tests ist es, über die Zeit von zwölf Minuten mit maximaler Geschwindigkeit zu laufen, da am Ende die zurückgelegte Strecke die entscheidende Meßgröße darstellt. Die Ausführung kann sowohl mit konstanter als auch mit variierender Geschwindigkeit stattfinden. Die Validität, ermittelt durch Korrelationen mit anderen Fitnesstests, liegt im Mittel über 0.80, was, angesichts der Korrelationen mit der Sauerstoffaufnahme (0.87 - 0.90; siehe Johnson, Oliver \& Terry, 1979, S.166) und den publizierten Gütekriterien (Rel.: 0.82 - 0.95; vgl. Bös, 1989) für ,,eine hohe Konstruktvalidität im Hinblick auf die adäquate Operationalisierung der kardiopulmonalen Ausdauer“" (Bös, 1989, S. 297) spricht.
} 
Sauerstoffaufnahme assoziierte Streckenwerte angibt, die Ausdauerscheibe entwickelt wurde (Fors, 1976).

Als Teil der Belastungstestung nimmt die kardio-zirkulatorische Funktionsprüfung eine herausragende Position ein (Nowacki, 1987, S. 244). Die Herzfrequenz steht in der Trainingspraxis nach wie vor an erster Stelle, wenn es um die Ermittlung des Leistungsstands (anhand der Herzfrequenzänderung unter definierten Belastungs- und Pausenbedingungen beurteilt) geht. Bei der organischen Leistungsfähigkeit steht die absolute und relative Sauerstoffaufnahme an führender Position, gefolgt von der Laktatmessung, die die Bestimmung der aerob-anaeroben Schwelle ermöglicht (vgl. Nowacki, 1987; kritisch hierzu: Bueno, 1990). Zu diesem Zweck eignet sich auch der Conconi-Test (vgl. Multerer \& Bös, 1989), der 'unblutig' diese Schwelle durch den Verlauf des Anstiegs der Herzfrequenz (Übergang von linearem zu exponentiellem Anstieg zweiten, dritten Grades) bei steigender Belastung erkennen läßt; der größeren Ungenauigkeit steht der wesentlich geringere Aufwand gegenüber. Ein weiterer Wert von aktueller und individueller Bedeutung ist die PWC 170 (physical working capacity), die wiederum in Watt ausgedrückt diejenige fahrradergometrische Leistung beschreibt, bei der eine Herzfrequenz von 170 Schlägen/min erreicht wird. Neben der Blutdruckmessung verbleiben noch die biochemischen Methoden, deren Existenz jedoch nur der Vollständigkeit halber erwähnt werden sollen (vgl. Neumann \& Schüler, 1994).

\subsubsection{Zum Begriff der Wahrnehmung}

\subsubsection{Allgemeine Merkmale der Wahrnehmung}

Wahrnehmung wird von dem Begriff der Empfindung unterschieden. Wahrnehmung beinhaltet schon eine gerichtete Aufmerksamkeit und ein aktives Aufnehmen ins Bewußtsein. Wahrnehmung dient der adäquaten Bewegungssteerung.

The senses lose their place as gateways of knowing to take their rightful place as stimuli to action. To an animal an affection of the eye or ear is not an idle piece of information about something indifferently going on in the world. It is an invitement and inducement to act in a needed way. (Dewey, 1920, S. 83)

Wie schon traditionell in der Philosophie und Physiologie wird auch in der Psychologie der ,... Begriff der Wahrnehmung deutlich vom Begriff der Empfindung abgehoben“ (Westermann, 1987, S. 265). Als Empfindung ('sensations') bezeichnet man dabei die elementarsten Bewußtseinsinhalte, die unterschiedlichen beobachtbaren Qualitäten innerhalb 
der einzelnen Sinnesmodalitäten oder die von den Sinnesorganen ausgehenden und kontrollierten Aktivitäten des Nervensystems (ebd.). Anders verhält es sich mit der Wahrnehmung. Diese beruht zwar auf den Empfindungen und ist unabdingbar mit diesen verbunden, sie kann aber weit über diese hinausgehen. „Wahrnehmung beinhaltet schon eine gerichtete Aufmerksamkeit und ein aktives Aufnehmen ins Bewußtsein sowie eine Zuordnung zu vorhandenen Kategorien, eine subjektive Repräsentation oder Deutung“ (Westermann, 1987, S. 265).

So wird deutlich, daß eine neutrale Wahrnehmung niemals existieren kann, da die objektiven Reize, jene also, die physikalisch meßbar sind, nur einen Teil der Wahrnehmung konstituieren (sicherlich liegt einer solchen Aussage ein entsprechendes Realitätsverständnis zugrunde; hierauf wird im Kapitel 2.3.2.2, S. 89, eingegangen). Die übrigen Anteile werden durch individualspezifische Merkmale des Wahrnehmenden gebildet. Dadurch sind sie für eine andere Person niemals zugänglich und nicht nachprüfbar. Interpretiert werden diese Empfindungen, indem das Gehirn ,... das, was in der Gegenwart geschieht, mit dem vergleicht, was sich in der Vergangenheit ereignet hat" (Lindsay \& Norman, 1981, S. 2; vgl. Kap. 2.1.3.2.2.2.2, S. 51).

Aus soziobiologischer Sicht wird der Schritt - weg von der objektiven Wahrnehmung, hin zu der subjektiven - vervollständigt und die Verbindung zu der Stellungnahme Deweys (1920; s. o.) hergestellt: Wahrnehmung ist nicht einmal daraufhin angelegt, objektives Wissen über die Welt zu vermitteln, sondern daraufhin, als Antwort auf eingehende Reize (auch kinästhetische und propriozeptive) die für das Überleben wichtigen Verhaltensweisen zu evozieren. Hierbei ist es unerheblich, ob die Wahrnehmung die reale Welt widerspiegelt oder im Sinne der instrumentellen Sicht der Hypothesengenerierung (vgl. Westermann, 1987, S. 6 f.) zutreffende Aussagen über zukünftiges Geschehen und Handlungen ermöglicht, unabhängig, ob es zu den intervenierenden Vorstellungen bzw. Wahrnehmungen eine reale Entsprechung gibt. „Gehirne ... sind keine Galerie von Weltbildern, sondern pragmatische Organe zur Erzeugung angemessenen Verhaltens. Demgemäß dient auch Wahrnehmung nicht einer Re-Präsentation der Umwelt, sondern steht im Dienste adäquater Bewegungssteuerung“ (Schlosser, 1995, S. 1242). 


\subsubsection{Die Wahrnehmung der Belastung}

Belastungswahrnehmung wird von Anstrengungsempfindung getrennt. Vier Modelle zur Leib-Seele-Problematik werden vorgestellt, die Geschichte der Belastungswahrnehmung geschildert. Das Rating der wahrgenommenen Belastung ('Rating of Perceived Exertion', RPE) stellt die meistgebrauchte Skala dar.

Im Gegensatz zur 'Anstrengungsempfindung', welche ,... relates to a feeling of physical or mental tiredness resulting in a diminishing performance capacity“ (Watt \& Grove, 1993, S. 226), wird Belastungswahrnehmung ('perceived exertion') als 'Gestalt' (Ehrenfels, 1890) verstanden, die sich in der individuellen Wahrnehmung aus den Faktoren „... heart rate; power output $\left(\mathrm{VO}_{2}\right)$; ventilatory minute volume; beta endorphin, catecholamine, and lactate production; and the individual's training status“ (Morgan, 1993, S. 1072) zusammensetzt. Eine der Anstrengung ähnliche Empfindung kommt bei der Belastungswahrnehmung nur bei hohen Intensitäten vor, bei geringerer Intensität wird die Belastung beispielsweise als ‘Aktivität' oder 'angenehmes Gefühl, etwas zu tun' (vgl. Watt \& Grove, 1993), empfunden. „There has been a tendency for psychologists to think of perceived exertion as a physiological construct, whereas physiologists have often regarded effort sense as a psychological phenomenon. However, .... the rating of perceived exertion ... has been conceptualized as a psychophysiological phenomenon“(Morgan, 1993, S. 1071 f.).

„Ignoramus - ignorabimus“, der wenig ermutigende Ausspruch von Du Bois-Reymond (vgl. Benesch, 1994), markiert in der Geschichte der Philosophie nur eine Position zu der Leib-Seele Problematik. Andere Autoren vertraten die Auffassungen, daß der Mensch durchaus Kenntnis darüber gewinnen kann, wie das Verhältnis von Geist und Körper vorzustellen sei, ohne das der Zusammenhang zwischen Belastung und Belastungswahrnehmung nicht verstanden werden kann. Im Laufe der Geschichte haben sich vier große Richtungen herausgebildet (vgl. Benesch, 1994, S. 478 f.):

Monismus: Kernpunkt des Monismus ist die alleinige Anerkennung einer (neuronalen) Substanz. Das 'Psychische' stellt lediglich ein Begleitphänomen dar. Vertreten wurde diese Sichtweise erstmals 1721 von Christian v. Wolff. Heute wird dieser Ansatz noch in Form eines Materialismus ('Emergenter Materialismus') von nordamerikanischen Neuropsychologen vertreten, der die Höherwertigkeit des Psychischen gegenüber dem Physischen jedoch nicht leugnet.

Dualismus: René Descartes unterschied eine 'res cogitans' von einer 'res extensa' und gründete damit die Sichtweise, die als Dualismus bezeichnet wird. Das Psychische stellt hiernach eine eigenständige und unabhängige Instanz dar. So kann sie Aussagen über die Wechselwirkungen zwischen Physis und Psyche machen, wie es vor allem im Zusammen- 
hang mit psychosomatischen Phänomenen geschieht. Über die Art der Wechselwirkung werden jedoch keine Annahmen gemacht.

Parallelismus: Eine Mittelstellung zwischen Monismus und Dualismus nimmt der Parallelismus ein. Er geht auf Theodor Fechner zurück, der ihn ,als Monismus mit dualistischem Einschlag in dem Bild formulierte, Psychisches und Physisches würden sich zueinander wie die Außen- und Innenseite einer Kugel verhalten“( (a. a. O., S. 479). Bis heute hat der Parallelismus eine eher metaphorische denn eine forschungspraktische Relevanz.

Triplexität: Zurückzuführen ist diese Dreiheit der psychophysischen Beziehung auf die von Aristoteles geprägten Formbegriffe 'Substanz' und 'Entelechie'. Relevanz gewinnt diese Sicht in der heutigen Zeit durch Teile der Theoretischen Neuropsychologie. Neuronale Muster sind als Muster von den elektrochemischen Trägerprozessen abhebbar und innerkörperlich übertragbar, sogar interferierend mit Umweltmustern. Psychische Relevanz erhalten sie durch die Musterbedeutung. Die Dreiheit Träger-Muster-Bedeutung ist gleichzeitig eine zweifache Höherstufung, weil die Muster teilunabhängig (arbiträr) vom neuronalen Geschehen sind und ferner als Muster ambige (wandelbare) Bedeutung erlangen können.

Die Erforschung der Gesetzmäßigkeiten psychophysischer Beziehungen begründete Theodor Fechner, der mit der Einführung des Begriffs 'Psychophysik' 1860 einen neuen Bereich in der damals erst entstandenen Psychologie gründete. Dieser setzt sich mit der „... Abhängigkeit der Ausprägung, Größe oder Intensität von subjektiven Empfindungen wie Lautheit, Helligkeit, Schwere und so fort einerseits[,] von der Ausprägung, Größe oder Intensität physikalischer Reizmerkmale wie Schalldruck, Lichtenergie, Gewicht und so fort andererseits“ (Westermann, 1987, S. 267) auseinander, wobei er „,... einer äußeren (Beobachtung von Reiz-Erlebensbeziehungen) eine innere Psychophysik (Beziehungsbestimmung des Geistigen zur körperlichen Innenwelt) gegenüberstellt“ (Benesch, 1994, S. 478).

Psychophysical scaling of perceived exertion derived from the development of psychophysical scaling of perceptual attributes (loudness, brightness, taste, smell and other fundamental sensory areas) by Stevens at Harvard in the 1950s .... and permitted the first comprehensive measurements of the perception of effort and exertion. (Watt \& Grove, 1993, S. 226) 
Der geschichtliche Beginn der Belastungswahrnehmung im engeren Sinne (vgl. Carton \& Rhodes, 1985) zur Zeit des ausklingenden neunzehnten Jahrhunderts war nur bedingt vielversprechend. Cattell und seine Mitarbeiter ließen verschiedene Personen an einem Handgerät Kraft in bestimmten Verhältnissen zu einer Standardkraft ausüben. Die Einschätzung der ausgeübten Kraft entsprach jedoch nur in geringem Maße der tatsächlichen. Da es an einer erklärenden Theorie mangelte, wurden die Versuche eingestellt. Etwa siebzig Jahre später versuchten Stevens und Mack (1959) mit Hilfe von drei der unter dem Punkt 'Verhältnisskalen' (s. u.) angeführten Vorgehensweisen, die Zusammenhänge zwischen eingeschätzter und tatsächlicher Kraft zu ergründen. Sie beobachteten, daß die wahrgenommene Belastung stärker zunahm als die tatsächliche Kraft. Dieser Zusammenhang war durch eine exponentiell ansteigende Funktion mit einem Exponenten zwischen 1.3 und $3.1 \mathrm{zu}$ beschreiben, der Mittelwert lag bei 1.7 (vgl. auch Banister, 1979, S. 144 ff.).

Hinsichtlich der Belastungsempfindung bei Ausdauerleistung unter Beanspruchung gröBerer Anteile der Muskelmasse entwickelte Borg 1962 (vgl. Monahan, 1988, S. 174 ff.) eine kategorielle Skala mit 21 Punkten, aus der sich in der Folge die RPE-Skala (s. u.) entwickelte.

Nach derzeitigem Stand sind vier verschiedene Techniken der Belastungswahrnehmungsskalen in der Literatur vertreten (Watt \& Grove, 1993, S. 226 ff.):

- Verhältnisskalen ('Ratio Scaling'), die nach einer Potenzfunktion mit Exponenten von 1.5 bis 1.9 am besten den Zusammenhang zwischen Wahrnehmung kurzzeitiger Anstrengung und aufgewandter Kraft beschreiben; die Schätzung des Zusammenhangs erfolgt hinsichtlich der Größe der aktuellen Belastung in bezug auf zuvor wahrgenommene Standardwerte, hinsichtlich des relativen Maßes wird das Verhältnis der Belastung zum Standard geschätzt. Bei der Reproduktion kehrt sich das Vorgehen um, nach vorgegebenen Größen oder Verhältnissen wird die Belastung selbst gewählt.

- Kategorielle Skalen ('Category Scaling') verlangen von der Person, die wahrgenommene Belastung, vor allem länger andauernde Arbeit, in Kategorien gleicher Abstände, die mit Zahlen bezeichnet sind, einzuordnen, nachdem die Endpunkte mit Beispielen verankert werden.

- Die Einschätzung akzeptabler Gewichte ('Ratings of Acceptable Load') entstammt der Arbeitswelt. Mit der Aufgabe, das schwerste - aber noch nicht zu schwere Gewicht hochzuheben, beginnt der Durchlauf beim ersten Mal mit einem leichten, beim zweiten 
Mal mit einem schweren Gewicht. Die Ergebnisse werden gemittelt und stellen die zumutbare Belastungsschwelle für die zu verrichtende körperliche Arbeit dar.

- Rating der wahrgenommenen Belastung ('Rating of Perceived Exertion', RPE) ist die meistgebrauchte Skala. Die RPE-Skala, ,... constructed to aid a person in estimation and regulating exercise intensity ...“ (Borg \& Ottoson, 1986, S. 6), ist eine 1962 von Borg und Dahström (Eston \& Williams, 1988) eingeführte Ratingskala, die - neben einigen verbalen Verankerungen lediglich die Zahlen von 6 bis 20 enthaltend - ,... an accepted method of assessing exercise intensity“ (Eston \& Williams, 1988, S. 153) darstellt. Mit dem Ziel, eine Basis für interindividuelle Vergleiche $\mathrm{zu}$ schaffen, „... interindividual comparisons have been applied especially to the modality of effort-perception ... [and] have been calculated according to the model and then validated against physiological effort responses, such as heart rates .... breathing frequencies, lactic acid concentration .... (Borg, 1974, S. 2). Damit ist es unabhängig vom Trainingszustand möglich, durch die Vorgabe gleicher Zahlen bei verschiedenen Personen die auf physiologische Parameter bezogene gleiche Belastungsintensität (z. B. 70 \% der ventilatorischen Schwelle; Dunbar, Robertson, Baun, Blandin, Metz, Burdett \& Goss, 1992) zu provozieren, oder aber nach Belastung durch den Vergleich der Werte Aussagen über die Belastung, unabhängig von der absoluten Leistung, machen zu können. Ein weiteres mit der Aufstellung dieser Skala verbundenes Anliegen war eine einfache Erfassung von physiologisch wichtigen Werten; sie ist so konzipiert, daß die Werte den um den Faktor 10 gekürzten Zahlen der Herzfrequenz entsprechen (z. B. RPE: $16 \approx$ Hf: 160 Schläge/min), was trotz oder dank ihrer Schlichtheit angesichts von Korrelationen zwischen diesen beiden Faktoren nahe 0.85 (Carton \& Rhodes, 1985, S. 204) als gelungen angesehen werden kann. Dadurch ist sie nicht zuletzt auch als Alternative zu der aufwendigeren Kontrolle durch die direkte Erhebung der physiologischen Parameter zur Trainingssteuerung geeignet.

Der Zusammenhang zwischen Belastung und Belastungswahrnehmung ist Gegenstand vieler Untersuchungen. Er soll hier exemplarisch an der RPE-Skala aufgezeigt werden. Wanner untersuchte 1985 Sportstudenten mit der zugrundeliegenden Fragestellung, ob die subjektive Einstufung der Belastung bei Ausdauerleistung mit der Veränderung der Belastung variiert. Die Einschätzung erfolgte in fünf Kategorien von 'sehr klein' bis 'sehr groß'; die Wahl der Kategorien geschah in Abhängigkeit von der Laufgeschwindigkeit bzw. der Belastungsintensität (Wanner, 1985, S. 108 ff.).

Die Überlegung, daß aufgrund verschiedener Trainingslevel eventuell unterschiedliche Einschätzungen der RPE vorgenommen werden, war der Ausgangspunkt, Personen unter- 
schiedlicher Trainingszustände mit vergleichbarer Belastung zu konfrontieren und deren Einschätzung der Belastung zu erheben. Es wurden jedoch keine signifikanten Unterschiede gefunden. Die Gruppe mit der weniger ausgeprägten Fitness gab die gleichen Belastungsempfindungen bei der individuellen Belastung von $90 \%$ der jeweiligen ventilatorischen Schwelle an wie die Hochtrainierten (Berry, Wyrich, Robergs, Krause \& Ingalls, 1989). Diese Befundlage entspricht der Mihevics, die einige Jahre zuvor mit der gleichen Fragestellung zu der Erkenntnis kam, daß „,... individuals differing in cardiovascular fitness rate their perceived exertion at absolute work loads similarly ..." (Mihevic, 1983, S. 242). Gleiches fanden auch Dishman, Graham, Holly und Tieman (1991), die die Einwirkung des 'Typ A'-Verhaltens (vgl. Peters, 1990, S. 544) auf die Angaben der RPE-Werte studierten.

Die Relation zu Laktatkonzentration und Herzfrequenz stand für Noble, Borg, Jacobs, Ceci und Kaiser (1983) im Vordergrund, die mit einer kleinen Stichprobe ( $n=10)$ eine sehr hohe Korrelation ( $\mathrm{r}=.99)$ zwischen Herzrate und Einschätzung auf der Borg-Skala ${ }^{8}$ beobachteten, während die Verbindung zum Laktat besser mit einer Exponentialfunktion mit einem Exponenten von $1.65 \pm 0.02$ zu beschreiben war (S. 525).

Eine ganze Untersuchungsreihe wurde von Fleishman, Gebhardt und Hogan (1984) zur Überprüfung von Reliabilität und Validität eines Anstrengungsindex durchgeführt. Die Belastung wurde durch Erhebung metabolischer Werte und Expertenratings objektiviert. Auch diese Autoren berichteten von einem engen Zusammenhang, ,taken together, these studies are encouraging with respect to the reliability and validity of the perceives effort index ...“ (Fleishman, Gebhardt \& Hogan, 1984, S. 951).

Die Einschätzung schließlich der Belastung als 'somewhat hard' (etwas anstrengend RPE 13) als Indikator für die aerob-anaerobe Schwelle ist der Erkenntniszuwachs einer Studie von Purvis und Cureton (1981). Dies gilt recht konsistent für die beteiligten Personen beiderlei Geschlechts.

\footnotetext{
${ }^{8}$ Sie arbeiteten mit einer neu entwickelten RPE-Skala (1982), die die Punkte 0 bis 10 umfaßt und exponentiell (bei Fahrradbelastung beispielsweise 1,6) zu der Belastung ansteigt (Carton \& Rhodes, 1985, S. 200 f.). Sie konnte die vorherige Skala jedoch nicht ablösen.
} 


\subsubsection{Belastungswahrnehmung als Steuergröße für Belastungsintensität}

Wie Studien zeigen, kann Belastungswahrnehmung auch zur Trainingssteuerung verwandt werden. In Anlehnung an die Hypothese des depressiven Realismus besteht auch auf diesem Gebiet die Tendenz zur Selbstüberschäzung.

Die Betrachtung unter diesem Aspekt folgt der Frage, inwieweit die Belastungswahrnehmung auch als steuerndes Element bei der Wahl unterschiedlicher Belastungsgrade dienen kann. Auch hier wird der Schwerpunkt auf die in der Literatur stärker vertretenen energetischen Aspekte gelegt.

Eine Möglichkeit ist die Vorgabe einer Stufe der RPE, diese „,... method assumes that a person can adjust the power output to match numerically anchored verbal expressions of effort“ (Eston \& Williams, 1988, S. 153), was sich gemäß der Überprüfung der erwähnten Autoren - ihre Messung umfaßte Sauerstoffaufnahme und Herzfrequenz - als konsistent mit der empirischen Realität erwies (a. a. O., S. 153 f.). Ähnliches belegen auch die Resultate einer Forschergruppe um Dunbar (1992). Unter Vorgabe der RPE-Werte und Messung der Sauerstoffaufnahme ,... the mean production accuracy was excellent both intra- and intermodally .... [und dies] consistent at both the $50 \%$ and $70 \% \mathrm{VO}_{2 \max }$ exercise intensity“ (Dunbar, Robertson, Baun, Blandin, Metz, Burdett \& Goss, 1992, S. 97).

Mit der Vorgabe '11' der Borg-Skala wurde an ,... 20 nicht speziell ausdauertrainierten männlichen und weiblichen Sportstudierenden ...“ (Buskies, Kläger \& Riedel, 1992, S. 248) Herzfrequenz und Laktat kontrolliert. Diese Vorgabe der Belastungsempfindung bewirkte ein Verbleiben der Laktatwerte in einem Bereich von $2 \mathrm{mmol} / \mathrm{l}$. Unter Vorgabe vier verschiedener RPE-Werte beobachteten Ward, Jackman und Galiano, daß ,... adults were slightly above the criterion at the lower prescription levels (RPE 7 and 10) and were close to the criterion at RPE 13 and 16“"(1991, S . 211).

Ausdrücklich empfohlen wird die Borg-Skala zur Belastungssteuerung von dem American College of Sports Medicine, um den von derselben zum Erhalt der Fitness empfohlenen Standard ${ }^{9} \mathrm{zu}$ erreichen; der angeratene Bereich entspricht in etwa den RPE-Werten von 12 bis 16 (Pollock, Jackson \& Foster, 1986, S. 161).

Eine Verzerrung in positiver Richtung, vergleichbar der im Rahmen des depressiven Realismus referierten Beobachtungen, ist auch im Sportgeschehen zu verzeichnen. Dabei soll nicht diejenige Verzerrung Beachtung finden, die sich in einem vergleichsweise stärkeren Ansteigen der Belastungsempfindung als dem der zu erbringenden Leistung ausdrückt,

\footnotetext{
${ }^{9}$ Belastungsmerkmale: 3 bis 5 Male/Woche, bei 60 bis $90 \%$ der maximalen Herzfrequenz, 20 bis 40 min Dauer, aerob
} 
da dieses Phänomen durch psychophysische Grundlagen determiniert scheint (vgl. Einführung in die Psychophysik, S. 60) und ihm durch die Beschreibung exponentieller Funktionen schon Rechnung getragen wurde. Vielmehr steht die Überschätzung von Leistungsvermögen und Fitness, unter Mißachtung von Körpersignalen, im Vordergrund.

Im Rahmen einer stationären Heilbehandlung, innerhalb derer es mit Patienten sporttherapeutische Ziele zu verwirklichen galt, bemerkten Pitroff, Bös und Wydra: ,... bei der Eingangsmessung lagen bei $80 \%$ der Versuchsgruppe und bei $90 \%$ der Kontrollgruppe die Laktatwerte, die als Indikator für die Belastungssteuerung aufgefaßt werden können, über dem Schwellenwert von 4 mmol/l“ (1988, S. 5). Ein Laktatwert von 4 mmol/l steht als Indikator für die aerob-anaerobe Schwelle und damit zugleich für eine physiologisch sinnvolle Belastung im Freizeitbereich. Diese Überschätzung wurde durch ein anschließendes Training in der Versuchsgruppe zu einer realistischen Einschätzung zurückgeführt. Mit Überschätzung ist jedoch in diesem Zusammenhang nicht diejenige gemeint, die in Folge der Vorgabe bestimmter Belastungswerte (z. B. RPE-Werte) auftritt, und bei der, wie im vorherigen Kapitel besprochen, die Umsetzung recht akkurat geschieht. Gemeint ist vielmehr jene, die durch die ungeübte Wahrnehmung der Körpersignale hervorgerufen wird.

„Die Messung der metabolischen Belastungsintensität anhand des Blutlaktatspiegels zeigte, daß sich die Freizeitsportler im Mittel viel zu intensiv belasten“, urteilte Völker (1984, S. 6), nachdem er Freizeitsportler erst beim normalen Sporttreiben, dann unter kontrollierten Bedingungen untersuchte. Läufer und Schwimmer wurden bei ihrem normalen Training beobachtet, wobei die Strecke und die Zeit festgehalten wurden. Im zweiten Durchgang führten die Freizeitsportler das gleiche Training unter kontrollierten Bedingungen durch, mit der Instruktion, sich hinsichtlich der Belastungsintensität - genauso wie sie es für das normale Training angegeben hatten - von ihrem Gefühl leiten zu lassen. Die Erhebung der Zeit und der Strecke in der natürlichen Bedingung d. h. die Ähnlichkeit dieser Werte mit denen, die unter Versuchsbedingung erhalten wurden, galten als Indikator für die Repräsentativität der Experimentalbedingung im Rahmen des unbeobachteten Trainings. Die unter der Experimentalbedingung gemessenen „... Belastungswerte von 7,1 \pm 2,7 $\mathrm{mmol} / \mathrm{l}$ für das Laufen und 8,0 \pm 3,4 $\mathrm{mmol} / \mathrm{l}$ für das Schwimmen müssen als wesentlich zu intensiv für ein Gesundheitstraining erörtert werden“(Völker, 1984, S. 6).

Krüger, der als Vereinsarzt Aerobicprogramme betreute, spricht im Rahmen der Belastungsüberprüfung anhand Herzfrequenzmessungen von einer ,,... gefährlichen Tendenz der Selbstüberschätzung“ (Krüger, 1985, S. 120), und auch Kindermann (1984) fand bei einer Untersuchung innerhalb derselben Sportart Blutlaktatwerte von 6 bis $9 \mathrm{mmol} / \mathrm{l}$, wobei er insbesondere auf die ,... zunehmende Acidose aufgrund der Lactatakkumulation während 
einer Übungsstunde [hinweist; diese] beeinträchtigt die neuromuskuläre Funktion und damit Koordination, was Verletzungen begünstigen kann“(Kindermann, 1984 S. 33). 


\subsection{Depression}

\subsubsection{Grundlagen der Depression}

Die Grundlagen der unipolaren Depression werden gemäß des Diagnoseschlüssels der Weltgesundheitsorganisation (ICD 10/WHO) in ihren Grundzïgen dargestellt und Erklärungsmodelle für die depressive Störung vorgestellt.

Auch wenn die aktuelle Forschung nahelegt, daß ,... depression is not a single disorder but rather a group of disorders heterogeneous with respect to symptoms, cause, course, therapy, and prevention“ (Abramson, Metalsky \& Alloy, 1989, S. 359; siehe auch Blockzitat zum Problem der Klassifikation, S. 69), erfolgt die Darstellung der unipolaren Depression, als ob sie ein einheitlicher syndromatologischer Komplex (s. u.) sei; eine Unterteilung in eventuelle Subtypen der Depression entspricht einer für die Ausrichtung der vorliegenden Untersuchung zu detaillierten Betrachtungsweise.

\subsubsection{Beschreibung der depressiven Störung}

Die Hauptsymptome der Depression bestehen in einer Veränderung der Stimmung oder der Affektivität, begleitet von inem Wechsel im Aktivitätsniveau, Interessensverlust, Freudlosigkeit, verminderter Energie, deutlicher Müdigkeit schon nach nur kleinen Anstrengungen, verminderter Konzentration und Aufmerksamkeit, vermindertem Selbstwertgefühl, Schuldgefühlen und Gefühlen von Wertlosigkeit, negativen Zukunftsperspektiven, Suizidgedanken, Schlafstörungen, vermindertem Appetit sowie vermindertem Selbstvertrauen.

Warum gibt Gott das Licht dem Mühseligen und das Leben den betrübten Herzen - die auf den Tod warten, und er kommt nicht, und nach ihm suchen mehr als nach Schätzen, die sich sehr freuten und fröhlich wären, wenn sie ein Grab bekämen - dem Mann, dessen Weg verborgen ist, dem Gott den Pfad ringsum verzäunt hat? .... Ich hatte keinen Frieden, keine Rast, keine Ruhe, da kam schon wieder ein Ungemach. (Hiob 3, 20-23.26)

Soweit die Verzweiflung Hiobs, einer Person der frühen Menschheitsgeschichte, die bereits an Symptomen litt, die über eine tiefe Traurigkeit hinaus gingen. Unruhe, Getriebenheit, vegetative Störungen und Schlaflosigkeit, die Hiob plagten (vgl. Fritze, 1987, S. 8), gelten heute als eng mit dem Begriff der Depression assoziiert.

Depression, im Volksmund oft undifferenziert mit der Traurigkeit gleichgesetzt, ,... may refer to a symptom, such as sadness, gloominess, low spirits, dejection ....[einerseits, andererseits] to a syndrom in which a variety of signs and symptoms typically occur in association with the mood change“ (Cameron, 1987, S. 3), was, um eine Ebene erweitert, auch Fritze (1987) in seiner Annäherung an die verschiedenen Abstraktionsebenen und Verwen- 
dungsarten des Depressionsbegriffs konstatiert: „Mit dem Begriff Depression kann ein Symptom, ein Syndrom oder ein nosologisches Konzept gemeint sein“ (S. 15).

Syndromatologisch orientiert, d. h. ,... das gemeinsame Auftreten von Symptomen ohne Rücksicht auf deren Entstehungsbedingungen“ (Möller, 1994, S. 8) beschreibend, geht der ICD-10 (Dilling, Mombour \& Schmidt, 1993) vor, der der allgemeingültige und -verbindliche Diagnoseschlüssel der Weltgesundheitsorganisation ist. Die depressiven Störungen sind in dem Kapitel 'F 3' (a. a. O., S. 129 ff.) als Teil der affektiven Störungen klassifiziert; dabei wird angestrebt, ,... zumindest teilweise einem 'atheoretischen' Ansatz folgend, auf Begriffsbildungen wie etwa Neurose, Psychose und Endogenität zu verzichten und diese durch Einführung einer deskriptiven, an diagnostischen Kriterien orientierten Klassifikation $\mathrm{zu}$ ersetzen .... [des weiteren] werden vor allem die depressiven Störungen nach Schweregrad und Verlauf neu eingeteilt“ (Dilling, Mombour \& Schmidt, 1993, S. 9).

Die Hauptsymptomatik (vgl. Dilling, Mombour \& Schmidt, 1993, S. 139 ff.), deren Dauer für eine entsprechende Diagnosestellung mindestens zwei Wochen betragen sollte, besteht bei der unipolaren depressiven Störung in einer Veränderung der Stimmung oder der Affektivität, begleitet von einem Wechsel im Aktivitätsniveau. Des weiteren leidet die Person oft unter Interessensverlust, Freudlosigkeit und verminderter Energie; deutliche Müdigkeit tritt oft nach nur kleinen Anstrengungen auf. Weitere, häufig zu beobachtende Symptome sind verminderte Konzentration und Aufmerksamkeit, vermindertes Selbstwertgefühl, Schuldgefühle und Gefühle von Wertlosigkeit, negative Zukunftsperspektiven, Suizidgedanken, Schlafstörungen, verminderter Appetit und vermindertes Selbstvertrauen.

Die gedrückte Stimmung ändert sich kaum, reagiert auch nicht auf Lebensumstände, unterliegt aber zum Teil charakteristischen Tagesschwankungen. Teilweise steht, da ein untypisches Erscheinungsbild durchaus üblich ist, Gequältsein, Angst oder motorische Unruhe mehr im Vordergrund als die sonst typischen (s. o.) Erscheinungsformen der Depression.

Bei zusätzlichem Vorhandensein des somatischen Syndroms - angesichts fragwürdiger wissenschaftlicher Absicherung auch mit den Begriffen melancholisch, vital, biologisch oder endogenomorph assoziierbar (a. a. O., S. 131) - kann es noch zu Einschränkungen der positiven Emotionalität, frühmorgendlichem Erwachen, dem Vorhandensein eines Morgentiefs, psychomotorischer Hemmung oder Agitiertheit, Gewichts- und Libidoverlust kommen. 
Die Klassifikation einer leichten, mittelschweren oder schweren depressiven Episode richtet sich nach Anzahl, Ausprägung und Kombination der Symptome. Bei den schweren reihen sich noch einige psychotische Momente ein (Dilling, Mombour \& Schmidt, 1993, S. 139 ff.). Für die unipolare Depression scheint im Durchschnitt eine Phasendauer von etwa fünf Monaten zu gelten. Verläuft die Störung periodisch, beträgt die Zykluslänge etwa fünf Jahre (Hautzinger \& de Jong-Meyer, 1994, S. 185).

Diese Beschreibung entspricht lediglich der Klassifikation des ICD-10 (Dilling, Mombour \& Schmidt, 1993), die zwar die wichtigste, aber nicht die einzige ist, die sich mit dem Komplex der Entstehung, der Aufrechterhaltung und den Erscheinungsformen der psychischen Störungen im allgemeinen und der depressiven im besonderen befaßt. Obwohl diese hinsichtlich der Klassifikationsversuche den momentanen, weltweiten Konsens darstellt und aus diesem Grund der vorliegenden Arbeit zugrunde gelegt wurde - gilt jedoch:

Die Beziehung zwischen Ätiologie, Symptomatik, zugrundeliegenden biochemischen Prozessen, Ansprechen auf Behandlungen und weiterem Verlauf bei affektiven Störungen sind gegenwärtig noch nicht soweit geklärt, daß ihre Klassifikation in einer für alle annehmbaren Weise möglich wäre. (Dilling, Mombour \& Schmidt, 1993, S. 131)

\subsubsection{Epidemiologie}

Die Prävalenzrate variiert mit dem Geschlecht. Bei Männern beträgt sie etwa $12 \%$, bei Frauen etwa $26 \%$.

Die Epidemiologie als ,... the study of the occurrence of disease and disorders in human population“ (Dean, 1985, S. 5) gibt einleitend einen Überblick über die zahlenmäßige Bedeutung der Depression.

Davison und Neale (1988) geben eine Prävalenz von 8 bis $11 \%$ für Männer und 18 bis $23 \%$ für Frauen an (S. 260), was sich annähernd mit der Einschätzung Hautzingers und de Jong-Meyers deckt, die von 12 bzw. $26 \%$ ausgehen (1994, S. 183). Auch für Wittchen und Zerssen (1988) liegen die Ergebnisse verschiedener Studien zwischen 15 und 20 \% (S. 1).

Auf die amerikanische Bevölkerung bezogen führt Dean (1985, S. XI) eine Prävalenz zwischen 8 und $18 \%$ an, eine in Oberbayern durchgeführte Studie fand ,...8,3\% of the 1536 respondents ... suffered from depressive disorders in need of treatment ..." (Weyerer, 1992, S. 493). Die Inzidenz, die auf ein Jahr bezogene Anzahl der Neuerkrankungen in einer Population, beläuft sich auf etwa 1 bis $2 \%$ (Hautzinger \& de Jong-Meyer, 1994, S. 182). 


\subsubsection{3 Ätiologische Modelle}

Biologische und psychologische Modelle werden eingeführt. Eine Übersicht zeigt zusammengefaßt die wichtigsten Faktren.

Bislang ist es noch keinem sich um eine Erklärung der depressiven Symptomatik bemühten Modell gelungen, alle Phänomene, die mit der Depression in Verbindung stehen, zu deuten. Manches Phänomen ist geklärt; und doch sind es nur kleine Ausschnitte, die zudem, trotz der verschiedenen Bemühungen um Integration, recht isoliert und unverbunden nebeneinander stehen.

\begin{tabular}{|c|c|c|c|}
\hline \multicolumn{2}{|c|}{ Biologische Faktoren: } & \multicolumn{2}{|c|}{ Psychologische Faktoren: } \\
\hline - Genetik & $\begin{array}{l}\text { Nebennierenrinden- } \\
\text { hormone }\end{array}$ & Persönlichkeit & $\begin{array}{l}\text { Dysfunktionale } \\
\text { Einstellungen }\end{array}$ \\
\hline $\begin{array}{l}\text { Monoamin- } \\
\text { oxidase }\end{array}$ & $\begin{array}{l}\text { Schilddrüsen- } \\
\text { hormone }\end{array}$ & Soziale Faktoren & $\begin{array}{l}\text { Hilflosigkeitserfahr- } \\
\text { ungen }\end{array}$ \\
\hline - Catecholamine & - Wachstumshormone & - Lebensereignisse & $\begin{array}{l}\text { Interaktionsmerk- } \\
\text { male }\end{array}$ \\
\hline - Acetylcholin & - Biorhythmik, Schlaf & $\begin{array}{l}\text { Aversive Lebensbe- } \\
\text { dingungen }\end{array}$ & $\begin{array}{l}\text { Multifaktorielle } \\
\text { Modelle }\end{array}$ \\
\hline - GABA & $\begin{array}{l}\text { Rezeptorveränder- } \\
\text { ung }\end{array}$ & Verstärkerverlust & \\
\hline
\end{tabular}

Abbildung 1: Übersicht über depressionsätiologisch bedeutsame Faktoren (vgl. Hautzinger \& de JongMeyer, 1994, S. 187)

\subsection{Biologische Faktoren}

Familien-, Zwillings- und Adoptionsstudien sind das bevorzugte Untersuchungsgut der Vertreter genetischer Modelle. Bei den biochemischen Hypothesen konkurrieren der Einfluß von Noradrenalin, Serotonin, Thyreotropin, GABA und andere Rezeptorsysteme miteinander. Die Hypothahmus-Hypophysen-Nebennierenrinden/Schilddrüsen-Achse wird dargestellt.

Mit dem Forschungsdesign der Familien-, Zwillings- und Adoptionsstudien versuchen Wissenschaftler im Bereich der Genetik, Aussagen über die Entstehung der Depression zu machen (vgl. Hautzinger \& de Jong-Meyer, 1994, S. 194 f.). Diese beziehen sich jedoch zum größten Teil auf beschreibende Merkmale, da über den Vererbungsgang selbst kaum Einigkeit besteht. Der nichtdeskriptive Konsens, dessen ungeachtet einige Forscher an der Idee des dominanten X-chromosomal gebundenden Erbgangs festhalten, besteht zur Zeit in einem polygenetischen Übertragungsmodell, das als einziges geeignet scheint, die der aktuellen Forschungslage entstammenden Befunde zu integrieren (vgl. Hautzinger \& de JongMeyer, 1994, S. 194 f.). 
Auf der deskriptiven Ebene findet lediglich eine Gegenüberstellung von Zahlen statt. Demzufolge liegt die Konkordanzrate bei monozygoten Zwillingen zwischen 30 und $80 \%$, bei dizygoten nur zwischen 0 und $30 \%$. Allgemeine psychopathologische Auffälligkeiten sind bei Adoptivgeschwistern nur in $8 \%$ der Fälle zu vermerken, während es bei leiblichen $66 \%$ sind. Das Lebenszeitrisiko hinsichtlich affektiver Störungen bei Angehörigen ersten Grades von Patienten mit affektiven Störungen ist im Durchschnitt etwa doppelt so hoch wie das der Allgemeinbevölkerung.

Ende der achtziger Jahre kristallisierte sich unter dem Label 'Linkagestudien' ein neuer Trend in der Genforschung heraus (vgl. Ehlers, o. Jahresangabe, S. 10), der sich auf die räumliche Nähe zweier Gen-Loci mit der daraus folgenden größeren Wahrscheinlichkeit einer gemeinsamen Vererbung bezieht. Untersuchungen an Personen des 'Old Order Amish' einer Gruppe von hohem wissenschaftlichen Interesse, da alle zwölftausend heute Lebenden von nur dreißig Ahnen abstammen und, dank sorgfältiger Stammbaumaufzeichnungen gute Rekonstruktionsmöglichkeiten der einzelnen Vererbungsgänge gegeben sind - ließen zuerst eine solche Nähe, und damit verbunden, eine Disposition für affektive Störungen auf dem elften Chromosom vermuten, was jedoch durch Folgeuntersuchungen nicht bestätigt werden konnte.

Die Biochemische Hypothese (vgl. Davison \& Neale, 1996, S. 269 ff.; Hautzinger \& de Jong-Meyer, 1994, S. 195 ff.) erschöpft sich nicht in der Postulierung eines stringenten Theoriegangs. Sie stellt vielmehr ein Konglomerat verschiedener Annahmen, Befunde und theoretischer Überlegungen dar.

Die Noradrenalin-Mangel-Hypothese entstammt der Beobachtung, daß etwa $15 \%$ der hypertonischen Patienten depressive Symptome entwickelten, nachdem sie zur Senkung des Blutdrucks mit dem die biogenen Amine reduzierenden Medikament 'Reserpin' behandelt wurden. Zugleich haben Wirkstoffe, die die Monoaminoxidase ${ }^{10}$ hemmen, antidepressive Wirkungen. Daraus leitete sich die Vermutung ab, daß ein Defizit des Noradrenalins an wichtigen Stellen im zentralnervösen Bereich depressionsätiologisch bedeutsam sein könnte, was in der Folgezeit eine intensive Beschäftigung der Forschungsgemeinschaft mit diesem Catecholamin hervorrief. Nachdem durch Stimulation des Locus coeruleus, einer

\footnotetext{
${ }^{10}$ Die Monoaminoxidase ist nur als eine der Möglichkeiten, die Menge des Noradrenalins im synaptischen Spalt zu beeinflussen, angeführt; letztlich spielt es eine untergeordnete Rolle, ob mit dem Ziel dieser Beeinflussung auf die Resynthese, die Freisetzung, die Speicherungsmöglichkeiten in den Vesikeln, die Empfindlichkeit der entsprechenden Rezeptoren oder auf den Abbau im synaptischen Spalt eingewirkt wird. Allerdings nur auf der Ebene der funktionalen Betrachtungsweise. Depressive reagieren durchaus uneinheitlich auf verschiedene, auf unterschiedliche Ansatzpunkte einwirkende Medikamente.
} 
der Syntheseorte dieses Catecholamins und über ein dichtes Geflecht von Nervensträngen mit dem Hippocampus, dem Hypothalamus und dem Cortex verbunden, bei Tieren depressionsähnliche Symptome hervorgerufen werden konnten, glaubte man, den Beweis für die ätiologische Relevanz des Noradrenalins geführt zu haben. In der Folge wurden jedoch hierzu konsistente wie auch inkonsistente Befunde veröffentlicht, so daß der letztendliche Beweis der ursächlichen Verantwortlichkeit des Noradrenalins für die depressive Problematik weiterhin aussteht. So wird auch von einer Multikausalität ausgegangen, indem Dysbalancen verschiedener Transmittersysteme als ursächliche Mechanismen zugrundegelegt werden. Das Serotonin, ein Monoamin ${ }^{11}$, bildet die Basis für noch uneinheitlichere Forschungsbefunde.

Einheitlicher verhält es sich mit der Betrachtung zweier neuroendokriner Systeme, die aus der Verbindung von Hypothalamus, Hypophyse und Nebennierenrinde bzw. Schilddrüse gebildet werden.

Ein Hinweis und zugleich spezifischer Indikator für die Überaktivität der Hypothalamus-Hypophysen-Nebennierenrinden-Achse bei (zumindest 'endogen') Depressiven ist die Reaktion auf die Einnahme von Dexamethason. Während normalerweise die Kortisolproduktion daraufhin mindestens einen Tag lang unterdrückt wird, haben Depressive sehr viel schneller wieder normale und damit überhöhte Werte. Dies entspricht auch dem schon länger bestehenden Wissen um allgemein erhöhte Kortisolwerte in verschiedenen Körperflüssigkeiten bei Depressiven. Diese Achse ${ }^{12}$ wird durch Noradrenalin und Kortisol (via negativer Rückkopplung) gehemmt, während die Catecholamine des Nebennierenmarks stimulierend auf die Ausschüttung des adrenocorticotropen Hormons einwirken (vgl. Silbernagl \& Despopoulos, 1991, S. 260 f.). Die Hoffnung, mit diesen Mechanismen ein sicheres Diagnosesystem zur Verfügung zu haben, blieb jedoch unerfüllt. Die Spezifität der durchgeführten Labortests schwankte zwischen 36 und 96 \% (Hautzinger \& de Jong-Meyer, 1994, S. 199).

In bezug auf die Hypothalamus-Hypophysen-Schilddrüsen-Achse ist vor allem das Phänomen auffällig, daß die bei Nichtdepressiven zu beobachtende nächtliche Ausschüttung des Thyreotropins bei Depressiven nicht oder zumindest nur vermindert stattfindet. Auch die exogene Gabe von Thyreoliberin, welches normalerweise vom Hypothalamus kom-

\footnotetext{
11 'Monoamin' als die Bezeichnung der um das Serotonin erweiterten Catecholamingruppe

${ }^{12} \mathrm{CRH}$ aus dem Hypothalamus bewirkt die Freisetzung von ACTH im Hypophysenvorderlappen, das wiederum aktivierend auf die Nebennierenrinde wirkt und dort einer circadianen Rhythmik folgend, neben Mineralkortikoiden und Androgenen, auch Kortisol abgibt (vgl. Silbernagl \& Despopoulos, 1991, S. 260 f.).
} 
mend die Thyreotropin-Ausschüttung stimuliert, verhilft Depressiven im Gegensatz zu Nichtdepressiven nicht zu einer deutlichen Erhöhung der Thyreotropinkonzentration. Doch bei einer Sensitivität der entsprechenden Tests von etwa $40 \%$ kann auch dieses System nicht zu einer eindeutigen Diagnose beitragen.

Andere diskutierte, aber bislang nur in Ansätzen validierte Hypothesen beschäftigen sich mit dem Gleich- bzw. Ungleichgewicht im adrenerg-cholinergen System, der Relevanz der Gammaaminobuttersäure, möglichen Rezeptorveränderungen im prä- und postsynaptischen Bereich (die den Befunden gerecht zu werden versuchen, daß Wirkungen von Antidepressiva oft erst nach einigen Tagen bis drei Wochen eintreten, wenn der Neurotransmitterspiegel aber schon wieder auf das Ausgangsniveau zurückgegangen ist) und beobachteten psychophysiologischen Normabweichungen, die als physiologisches Korrelat der Depression betrachtet werden (vgl. Giedke, 1988, S. 133 ff.).

\subsection{Psychologische Faktoren}

Verschiedene Theorien haben sich um die Erklärung der depressiven Störung bemüht. Nach Abraham und Freud ist die 'Ichzentriertheit' der Faktor, der aus normaler Trauer eine Depression entstehen läßt. Seligmann sieht die Ätiologie in Analogie zu der gelernten Hilflosigkeit, Abramson erweitert das Konstrukt zur 'Hoffnungslosigkeit'. Beck macht Schemta, kognitive Fehler und die kognitive Triade für die Entstehung der Depression verantwortlich. Lewinsohn postuliert den Kernpunkt des Verstärkerverlusts. Die Betrachtung soziologischer Faktoren steht bei Brown im Vordergrund. Hautzinger und Lewinsohn präsentieren ein multifaktorielles Erklärungsmodell, das die Mehrzahl der anderen Annahmen intgriert.

Ein Merkmal der Psychologie - das Denken in unterschiedlichen Modellen auf verschiedenen, sich überlappenden, aber einander nur selten berührenden Ebenen (d. h. 'multiparadigmatisch' im Sinne Kuhns, 1962; vgl. auch Stegmüller, 1979, S. 142 ff.) - prägt die folgenden Ausführungen. Es erfolgt ein kurzer Abriß der wichtigsten Theorien, die sich um die psychologische Erklärung der depressiven Störung bemüht haben.

Der Unterschied zwischen Trauer und Depression, der im Volksmund, die beiden Ausdrücke synonym gebrauchend, oft nivelliert wird, spielt bei Abraham in der psychoanalytischen Annäherung an den Komplex der Depression eine zentrale Rolle (vgl. Davison \& Neale, 1996, S. 257 ff.); am Scheitelpunkt beider Wege steht sowohl nach seiner Ansicht als auch nach der Siegmund Freuds die 'Ichzentriertheit' (ebd.).

Die Entstehung der Depression wurzelt in der oralen Phase, in der die Bedürfnisse des später Depressiven nicht bzw. übermäßig befriedigt werden. Dadurch persistiert die betroffene Person in dieser Phase und bleibt auch zukünftig in ihren Wunscherfüllungen auf die für diese Phase typischen Befriedigungsmöglichkeiten angewiesen und in ihrem Selbstwert von dem Urteil anderer abhängig. 
Der Schritt zu der manifestierten Depression im fortgeschrittenen Alter vollzieht sich ungleich komplexer. Bei jedem Trauerakt, so Freud, wird das Objekt des Verlusts introjiziert, um wenigstens in Teilen diesen Verlust ungeschehen zu machen; da aber in jeder von Liebe geprägten Beziehung auch negative Gefühle gegenüber dem anderen bestehen, wird „... der Trauernde durch den Akt der Introjektion zum Objekt seines eigenen Hasses ...“ (Davison \& Neale, 1996, S. 258). Dies wird durch Gefühle der Wut, verlassen worden zu sein, und die der Schuld, hervorgerufen durch imaginäres oder tatsächlich dem Verlorenen zugefügtes Böses, begleitet. Die Zeit der Trauerarbeit, in der der Betroffene sich mit dem Verlust auseinandersetzt und sich von den durch die Introjektion konstituierten engen Banden löst, beginnt.

An dieser Stelle scheiden sich die Wege, der erwähnte Schritt kann vollzogen werden: Der nichtpathologische Weg führt zu einem Ende der Trauerarbeit, die, wenn sie gelungen ist, die Ablösung vom Objekt des Verlusts bewirkt. Der andere, begangen von einem im Übermaß Abhängigen, mündet in einen Prozeß der ,... Selbstverachtung, Selbstbeschuldigung und Depression ..." (Davison \& Neale, 1996, S. 258); der nach innen gerichtete, auf die eigene Person bezogene Zorn ist das psychoanalytische Substrat der Depression. Um Depressionen bei Personen ohne ein Verlusterlebnis mit in das Konstrukt integrieren zu können, wird der 'symbolische Verlust' als dem tatsächlichen gleichwertig postuliert, hierbei ist ,... das Objekt ... nicht etwa real gestorben, aber es ist als Liebesobjekt verlorengegangen“ (Freud, 1917/1946, S. 431).

Versuche, in denen Tieren extreme motivationale und emotionale Defizite induziert wurden, bildeten den Grund- und Eckstein der in den siebziger Jahren von Seligman postulierten Theorie der Gelernten Hilflosigkeit, die im Verlauf zunehmend zu dem Kreis der wichtigsten depressionsätiologischen Ansätze gerechnet wurde und als ,perhaps the most successful application of a nonclinically derived explanation of depression ..." (Sweeny, Anderson \& Baily, 1986, S. 974) eingeschätzt wird.

Bei Hunden, und in der Folge auch bei Ratten und anderen Tieren, wurde nach einer Behandlung mit aversiven Reizen in einer Situation, in der die Tiere diesen Reizen nicht entfliehen konnten, ein apathisches Verhalten und eine herabgesetzte Fähigkeit zum Lernen beobachtet, die die Tiere ohne diese Vorbehandlung nicht aufwiesen. Obwohl sie in einer darauf folgenden Versuchsanordnung den dort vorhandenen unangenehmen Reizen hätten entfliehen können, verblieben sie an ihrem Ort. Sie hatten Hilflosigkeit gelernt.

Die Theorie, die in ihrer Übertragung auf den Menschen zu einem ätiologischen Modell für die Depression wurde, postuliert einen Prozeß in drei Stufen, nach dem zuerst Informa- 
tionen hinsichtlich der Kontingenz zwischen Umweltereignissen und Reaktionen eingeholt werden, die gefundene Nichtkontingenz sich in der Erwartung auch zukünftiger Unabhängigkeit von Verhalten und Verstärkung niederschlägt und schließlich zukünftiges Lernen negativ beeinflußt wird (vgl. Weiner, 1988). Die Erfahrung der Nichtkontingenz - oder das damit verbundene, der mangelnden Beeinflußbarkeit relevanter Umwelteinflüsse entspringende Gefühl der Hilflosigkeit (a. a. O., S. 208) - führt zu depressiven Symptomen kognitiver, emotionaler, und damit auch motivationaler Art, mit Folgen im vegetativen und physiologischen Bereich (vgl. Hautzinger \& de Jong-Meyer, 1994, S. 187 ff.).

Eine Reformulierung erfuhr das Modell im Jahr 1978 durch Abramson, Seligman und Teasdale, die in dem Bestreben, die teilweise engen Grenzen und realitätskonträren Implikationen der Anwendung des in der klassischen Lerntheorie wurzelnden Modells auf die menschliche Depression auszuweiten, Attributionen in den Theoriemittelpunkt rückten (vgl. Davison \& Neale, 1996). Nicht mehr das Ereignis als solches war entscheidend, sondern, gemäß des phänomenologischen Ansatzes (vgl. Weiner, 1988, S. 311 ff.), die Wahrnehmung des Ereignisses bzw. die durch individuelle Attribution gesteuerte Interpretation desselben.

Hierbei sind vor allem drei Achsen von Bedeutung, entlang derer die Attributionen vorgenommen werden, wobei jede mit einer spezifischen Wirkung assoziiert ist. Die Dimension der Lokalisation, mit den Endpolen 'internal' und 'external', hat eine Wirkung im Bereich des Selbstwertgefühls; die Wahrnehmung des Kontrolldefizits innerhalb der Person hierbei wird eine internale Attribution vorgenommen - hat eine Verringerung des Selbstwertgefühls zur Folge. Die Stabilitätsdimension bestimmt die Dauer der Depression, so daß die Wahrnehmung eines negativen Umstands als 'stabil' diese verlängert. Die dritte Dimension, die der Generalität, beeinflußt die Symptombreite bzw. -vielfalt der Depression; Attributionen auf spezifische Faktoren machen nur partiell 'hilflos' (das Gefühl, relevante Ereignisse nicht beeinflussen zu können, bezieht sich nur auf spezifische Situationen), während globale Attributionen für das Gefühl einer über viele Situationen hinweg generalisierten Hilflosigkeit verantwortlich sind (Sweeny, Anderson \& Baily, 1986, S. 974 f.; vgl. auch die Einführung in Kap. 2.3.2.3.3, S. 112).

In einer weiteren Reformulierung (Abramson, Metalsky \& Alloy, 1989) wurde die 'Hilflosigkeit' in 'Hoffnungslosigkeit' umbenannt; ,... a proximal sufficient cause of the symptoms of hopelessness depression is an expectation that highly desired outcomes will not occur or that highly aversive outcomes will occur coupled with an expectation that no response in one's repertoire will change the likelihood of occurence of these out- 
comes .... The evolution from a helplessness to a hopelessness theory is ... that hopelessness, not helplessness, is a cause of the symptoms of depression“ (a. a. O., S. 359).

Das wissenschaftliche Paradigma - (im Sinne von Kuhn, 1962) -, das das Kognitive Modell der Depression einschließt, beinhaltet viel mehr als eine Theorie und eine Therapie. Es umfaßt auch einen bis vor kurzem noch außer acht gelassenen Bereich (die kognitive Organisation), eine Technologie und begriffliche Instrumente zur Datenerhebung in diesem Bereich, einen Satz allgemein anerkannter Prinzipien, um Beweise für die Absicherung der Theorie zu sammeln und zu bewerten. .... Genaugenommen hat sich in der kognitiven Organisation des Depressiven eine Revolution ereignet, die eine deutliche Umkehrung der Art und Weise bewirkt, in der der Patient die Realität konstruiert. Im Gegensatz zur herkömmlichen Beurteilung einer wissenschaftlichen Revolution, die gewöhnlich als Fortschritt betrachtet wird, stellt die ‘depressive Revolution' eine Regression dar. (Beck, Rush, Shaw \& Emery, 1994, S. 52; vgl. auch Blockzitat zum Begriff des Paradigmas, S. 89)

Um das ,... psychische Substrat der Depressionen zu erklären“ (Beck, Rush, Shaw \& Emery, 1994, S. 41), bemüht das kognitive Modell von Beck, entstanden in den sechziger Jahren, einen engen Verbund dreier Annahmen: Die kognitive Triade, Schemata und kognitive Fehler.

Die kognitive Triade beinhaltet wiederum drei Elemente. Das negative Selbstbild, der erste Bestandteil, führt den Depressiven dazu, sich als krank, unzulänglich, fehlerhaft oder benachteiligt zu sehen. Er schreibt unangenehme Erfahrungen „... einem psychischen, moralischen oder physischen Mangel seiner selbst ...“ (Beck, Rush, Shaw \& Emery, 1994, S. 41) zu. Des weiteren fehlen ihm nach seiner Einschätzung die Eigenschaften und Kompetenzen, die wesentlich wären, um Glück und Zufriedenheit zu erlangen. Der zweite Bestandteil entspricht der Neigung der depressiven Person, Erfahrungen negativ zu interpretieren; sie lebt, als ob die 'Welt' unangemessen viel von ihr verlange, ihr Hindernisse in den Weg lege oder in jedem Winkel des Lebens Enttäuschungen und Niederlagen bereithalte. Schließlich besteht eine negative Erwartung, die eine Zukunft antizipiert, die in der Sicht des Depressiven wiederum auch nur dessen momentane Probleme und Schwierigkeiten bietet.

Die Schemata als „,... stabile kognitive Verarbeitungsmuster“ (Beck, Rush, Shaw \& Emery, 1994, S. 43) erklären die Perseveration des Depressiven in der verzerrten, ihm schadenden Realitätswahrnehmung, selbst wenn ihm objektive Beweise für die Evidenz 
positiver Lebensaspekte potentiell zugänglich sind. Generell sind Schemata innerhalb der Fülle an Reizen, die jeder Situation innewohnen, für die Selektion verantwortlich und wandeln diese als mentale Repräsentation der Realität in Kognitionen um (vgl. auch SchemaDefinition nach Markus (1977), S. 127). So besteht trotz der vielen Möglichkeiten, Situationen interpretierend wahrzunehmen, aufgrund des Umstands, in ähnlichen Situationen ähnliche Schemata zu aktivieren, die Tendenz, auf Ereignistypen intraindividuell recht konsistent zu reagieren.

Personen mit einer depressiven Symptomatik, charakterisiert durch den ,,... systematic bias against the self...“ (Alloy \& Abramson, 1988, S. 225), passen die vorhandenen Informationen in zunehmendem Maße den negativen Schemata an, die immer generalisierter und dadurch mächtiger werden, so daß sie nach einiger Zeit bereits von recht entfernten Reizen aktiviert werden können. Gleichzeitig haben sie aber durch ihre verzerrte Realitätssicht kaum mehr die Möglichkeit, der Verzerrung gewahr zu werden. Erworben wurden diese negativen Schemata zumeist in der Kindheit und Jugend, beispielsweise durch Verlust eines Elternteils, durch Schicksalsschläge oder aufgrund erfahrener Zurückweisung durch Eltern oder andere Personen (Davison \& Neale, 1996, S. 259).

Die dritte Annahme besteht in den 'kognitiven Fehlern', die vermittelnd zwischen irrigen Annahmen und der Realität dem Depressiven diejenigen Nischen zur Verfügung stellen, in denen er nicht zur Aufgabe vertrauter Konzepte gezwungen wird. Die wichtigsten Fehler sind (nach Beck, Rush, Shaw \& Emery, 1994, S. 44 f.):

- Willkürliche Schlußfolgerung: Bestimmte Schlüsse werden trotz gegensetzlicher Beweise gezogen

- Selektive Verallgemeinerung: Ein aus dem Zusammenhang gerissenes Detail wird zur Grundlage für die Interpretation der Gesamtsituation

- Übergeneralisierung: Die Anwendung von aus wenigen Merkmalen einer bestimmten Situation entwickelten Regeln auf andere, kaum noch ähnliche Situationstypen

- Maximierung bzw. Minimierung: Die Verzerrung durch eine nichtadäquate Einschätzung der Bedeutung einer Situation

- Personalisierung: Die Tendenz, ohne tatsächlichen Grund den Bezug von Ereignissen zu der eigenen Person herzustellen 
- Verabsolutierung oder 'dichotomes Denken': Jede Beurteilung wird nur anhand der gegensätzlichen Kategorien bzw. Pole einer Dimension vorgenommen, Schattierungen, Zwischenformen oder Abstufungen werden negiert ${ }^{13}$.

Ferner lassen sich noch einige Merkmale identifizieren, die sich in Analogie zu Theorien Piagets hinsichtlich kindlicher Denkformen (vgl. Montada, 1987, S. 413 ff.) als Kennzeichen unreifer Denkformen herauskristallisiert haben (vgl. Beck, Rush, Shaw \& Emery, 1994, S.46). Diese Merkmale sind aufgrund ihrer Wichtigkeit mit Beispielsätzen dargestellt:

- Eindimensional und global:

Reduktionistische Beschreibung der eigenen Person auf nur einer Dimension „Ich bin ängstlich“ anstatt „Ich bin nett, etwas ängstlich und ziemlich faul“

- Invariabel:

Konstanz von Situationen und Charaktereigenschaften

„Ich war immer ängstlich und werde es immer sein“"

- Verabsolutierend und moralistisch:

Negativ wertende Beurteilung, immer die gesamte Person betreffend „Ich bin ein jämmerlicher Feigling“ anstatt „Ich bin vielleicht etwas ängstlicher als andere“

- Charakterdiagnose:

Denken in festgefügten Charakterzügen anstatt in variableren Verhaltensweisen „Ich bin ein Feigling“ anstatt „Ich habe mich in dieser Situation feige verhalten“

\section{- Irreversibel:}

Mangelnder Glaube an Veränderung

„Da ich von Grund auf feige bin, werde ich nie mutiger werden“

In bezug auf die Frage der depressiven Veranlagung besagt die Theorie, daß „,... frühe Erfahrungen die Grundlage für die Entstehung negativer Konzepte über das Selbst, die Zukunft und die Umwelt bilden. Diese negativen Konzepte (Schemata) können latent vorliegen, können aber durch besondere Umstände reaktiviert werden, die den ursprünglich für die Entstehung der negativen Einstellungen verantwortlichen Erfahrungen analog sind“ (Beck, Rush, Shaw \& Emery, 1994, S. 47).

\footnotetext{
13 'Schwarz-weiß-Denken'
} 
Menschen werden also depressiv, weil sie charakteristische logische Denkfehler begehen; so kehrt sich die durch andere Modelle nahegelegte Kausalität um. Der Depressive ist hier nicht das Opfer seiner Gefühle, sondern ,... seiner eigenen unlogischen Selbstbeurteilungen“ (Davison \& Neale, 1996, S. 260), aus denen daraufhin erst die mit der Depression assoziierten Gefühle entstehen.

Aus den operanten Lerntheorien (siehe S. 51) entstand Mitte der siebziger Jahre das Depressionsmodell von Lewinsohn, das die Ätiologie der Depression durch Verstärkungsverlust erklärt. Demgemäß reicht das Erleben reduzierter verhaltenskontingenter Verstärkung aus, um eine depressive Störung erstmalig auszubilden (vgl. Werner \& Rehm, 1975). In der Folgezeit wird das Verbleiben in der Krankheit durch die erhöhte soziale Zuwendung, die sich auf die Person als leidende, anstatt auf bestimmte, krankheitsunabhängige Persönlichkeitsmerkmale bezieht, zumindest kurzfristig verstärkt. Langfristig entsteht ein sich selbst nährender Kreislauf aus der Abnahme instrumenteller Aktivität des Individuums aufgrund weniger verhaltenskontingenter positiver Verstärkung einerseits und der negativen Verstärkung aufgrund reduzierter, potentiell zu verstärkender Handlungen anderseits, aus dem die betroffene Person allein nicht mehr herauszukommen vermag. Dieser Gedankengang ist konsistent mit einigen empirischen Befunden, gemäß derer, wie in der Besprechung der Studien zur sozialen Interaktion noch deutlich werden wird (vgl. S. 123), Depressive teilweise auch außerhalb ihrer durch die von Beck hypostasierte ${ }^{14}$ verzerrte Wahrnehmung geprägten subjektiven Realität in der Tat weniger soziale Verstärkung erleben als Nichtdepressive. Das potentielle Ausmaß der Verstärkung sieht Lewinsohn durch drei Faktoren bestimmt (vgl. Davision \& Neale, 1988, S. 275 f.): Persönliche Eigenschaften wie Alter, Geschlecht oder Lebensgeschichte, die Umgebung, in der der Betreffende lebt, und das Repertoire an Verhaltensweisen, die potentiell eine Verstärkung ermöglichen.

Die Betrachtung soziologischer Faktoren steht in verschiedenen Theorieansätzen im Vordergrund, die sich, wie auch Browns soziologisches Depressionsmodell (vgl. Hautzinger \& de Jong-Meyer, 1994, S. 191 f.), hauptsächlich auf die Beschreibung depressogener Lebensumstände stützen. Nach Brown sind drei Faktoren an dem depressiven Geschehen beteiligt: während die auslösenden Faktoren den Eintritt in die Depression steuern, bestimmen die Vulnerabilitätsfaktoren, welche Ereignisse depressionsrelevant sind und die symptomformenden Faktoren determinieren die Schwere der Störung. Als auslösende Faktoren fan-

\footnotetext{
${ }^{14}$ Angesichts der Uneinheitlichkeit, in der dieser Begriff verwendet wird, sei hier auf Wahrig (1986) verwiesen, demgemäß eine mögliche Definition ,(als vorhanden) unterstellen“ lautet (S. 678).
} 
den Brown und Harris (vgl. Wittchen \& Zerssen, 1988, S. 40 ff.) aversive Lebensereignisse, chronische Lebensschwierigkeiten und die Zugehörigkeit zur sozialen Unterschicht. Als Vulnerabilitätsfaktoren wirkten mangelnde intime bzw. emotional unterstützende Sozialbeziehungen, Verlust der Mutter vor dem elften Lebensjahr, Arbeitslosigkeit und der Umstand, mehr als drei Kinder unter vierzehn Jahren zu haben. Auch andere Autoren berichten von der Wirkung, die ,... life events, chronic strain, social support, and mastery ...“ (Turner, Noh \& Levin, 1985, S. 38) ausüben, wobei die Variable der sozialen Unterstützung zunehmend in das Zentrum der Untersuchungen rückt.

Weitere Modelle (vgl. Hautzinger \& de Jong-Meyer, 1994, S. 192) beschäftigen sich mit dispositionellen Faktoren (Persönlichkeitsvariablen), innerpsychischen Mechanismen (Selbstaufmerksamkeit, Lageorientierung), Bewältigungsstrategien nach aversiven Erfahrungen, Selbstregulationsmechanismen, dem Anspruchsniveau, dem Ausmaß sozialer Fertigkeiten, emotionaler Labilität, geringer Externalität und dem Ausmaß an Bereitschaft zu Selbstkritik.

$\mathrm{Zu}$ den integrativen Ansätzen gehören das von Hautzinger und Lewinsohn (vgl. Hautzinger \& de Jong-Meyer, 1994, S. 192 ff.) entwickelte 'Multifaktorielle Erklärungsmodell' und das 'Final-Common-Pathway-Model' (vgl. Hautzinger \& de Jong-Meyer, 1994, S. 202), ursprünglich entwickelt von Akiskal und McKinney (1975), erweitert durch de Jong (1987).

Entsprechend dem ersten Ansatz steht über allem die Prädisposition, die sich aus Faktoren wie Vulnerabilität, Persönlichkeit, Vorgeschichte, Immunität (durch Bewältigungsstrategien, positive Aktionen) und Umgebungsbedingungen zusammensetzt und in fast allen Stadien der Depressionsgenese ihre Wirkung entfalten kann. Die Genese der Depression als solche hat ihren Ausgangspunkt in auslösenden Ereignissen (vgl. Hautzinger \& de JongMeyer, 1994, S. 202) und unterbricht in der Folge automatisierte Verhaltensmuster, mit der Konsequenz einer Abnahme positiver Verstärkung und einer Zunahme aversiver Erfahrungen. Damit verbunden steigert sich die Selbstaufmerksamkeit (selbstkritische Betrachtung der eigenen Situation, Lageorientierung), es kommt zu einer reaktiven depressiven Verstimmung mit den entsprechenden emotionalen, kognitiven, verhaltensorientierten, sozialen und somatischen Konsequenzen, die in die depressive Störung münden können.

Das 'Final-Common-Pathway-Model' bemüht eine Vielzahl unterschiedlicher Systeme, Faktoren und Interaktionen verschiedenster Ebenen, um die Ätiologie der Depression verständlich zu machen. In abgewandelter oder direkt übernommener Form kommen viele der schon besprochenen Aspekte erneut zur Geltung. Der frühkindliche Objektverlust als eine 
der Psychoanalyse entstammende Einflußgröße steht in diesem Modell neben dem soziologischen Faktor der Weiblichkeit und dem biochemischen der Schilddrüsenunterfunktion. Die große Zahl an prädisponierenden Faktoren und Stressoren wird via intermediierender Biochemie in den 'final common pathway' geleitet, der über Regulationsstörungen ,... in den diencephalen Mechanismen der Verstärkung“ (Hautzinger \& de Jong-Meyer, 1994, S. 202) zur primären Depression führt.

\subsubsection{Exkurs I: Sport und Depression}

Viele Studien belegen einen positiven Einfluß von Sport auf Depression. Neurotransmitter, kardiovaskuläre und psychlogische Effekte sowie anthropologische Ansätze werden zur Erklärung angeführt. Eine Analogie zur Psychosomatik wird gebildet.

Mens sana in corpore sano.

Juvenal, 1. Jh. n. Chr.

Da die vorliegende Untersuchung mit Rehabilitationspatienten durchgeführt wurde (vgl. Kap. 3.2, S. 140), deren Rehabilitation die körperliche Betätigung zwingend beinhaltet (Eigentraining mit Stepper und Fahrradergometer, Krankengymnastik und Bewegungsspiele in der Gruppe), umreißt dieser Exkurs die eventuellen Wechselwirkungen zwischen Depression und körperlicher Aktivität. Die Relevanz für die eigene Erhebung ist jedoch eher gering, da selbst bei eventuell bestehenden positiven Effekten von Sport auf Depression ein daraus folgender systematischer Einfluß nichts daran ändern würde, daß eine in der konkreten Situation der Vorhersage zum ersten Meßzeitpunkt de facto vorhandene (subklinische) Depression potentiell die besagten Auswirkungen (vgl. Kap. 3.1, S. 134) mit sich bringen würde.

„Exercise can be associated with a decreased level of ... depression“ (International Society of Sport Psychology, 1992, S. 96) lautet das abschließende Urteil der ISSP hinsichtlich des psychologischen Nutzens körperlicher Aktivität, das insgesamt gültig, aber nicht in jedem Fall bestätigt zu sein scheint (für einen Überblick siehe Knobloch, 1993; Marti, 1992; Schwenkmezger, 1985). Williams und Getty (1986) beispielsweise fanden in einer über vierhundert Studenten involvierenden Studie ,... that $10 \mathrm{wk}$. of aerobic exercise ... which results in a significant improvement in cardiovascular functioning has no more effect on psychological mood states than does nonaerobic recreational games or no exercise and sport participation“ (1986, S. 1104). Auch Reiner, Kleine und Hautzinger resümieren nach einer Lauftherapie: „... es gelang nicht, auch nur einen Meßwert unter den Grenzwert 
von Depressivität zu senken“ (1990, S. 98). Golz (1991) hingegen berichtet im Rahmen des Berliner Sportprogramms, in dessen Mittelpunkt neben diversen Übungs- und Spielformen das Laufen stand, wie auch bei einer Kontrolle, die ein Jahr später stattfand, von deutlichen und stabilen depressionsmindernden Effekten (1991, S. 23). Zu vergleichbaren Ergebnissen kamen Brandon und Loftin (1991), die eine „,... positive association between physical fitness and various measures of emotionality“"(S. 567) fanden. Die Korrelation der Fitness mit dem Depressionsgrad betrug $r=-.52$.

Die Erklärungsansätze für eventuelle Effekte sind zahlreich. Neben eher unspezifischen Ansätzen, denengemäß Laufen eine Meditationsform darstellt oder in das Paradigma der Psychoanalyse eingebunden seine depressionsmindernde Wirkungen entfaltet (vgl. Sachs \& Buffone, 1984), orientiert sich einer der drei größeren Ansätze an physiologischen Prozessen.

Im besonderen haben hier die Endorphine, die „... sich mit den gleichen zentralnervösen Membranrezeptoren verbinden wie das Morphin und andere Opiate, also 'körpereigenes' Morphin darstellen ..." (Schmidt \& Thews, 1985, S. 73), durch ihre den Schmerz dämpfende und Wohlbefinden auslösende Wirkung Interesse geweckt, nachdem Langstreckenläufer von ekstatischen Zuständen während des Laufs berichtet hatten (vgl. Wildmann \& Krüger, 1986). Da sie jedoch in bedeutsamen Konzentrationen eher spät und erst nach großer Belastung ausgeschüttet werden, kamen sie in der Folge hinsichtlich ihrer Wirkung im Rahmen der nicht an Hochleistungssport angelehnten Körpertherapie depressiver Personen zunehmend weniger in Betracht.

Die Neurotransmitter Noradrenalin, Serotonin und Dopamin, die schon durch geringe Belastung vermehrt produziert werden, verdienen Beachtung. Die depressionsätiologische Bedeutung dieser Transmittersysteme wurde bereits an anderer Stelle eingehend erwähnt (vgl. S. 71). Da körperliche Aktivität eine erhöhte Ausschüttung dieser Neurotransmitter provoziert, ist die Wirkungsweise sportlicher Betätigung der von Psychopharmaka vergleichbar, die ihrerseits mit den ihnen zur Verfügung stehenden Mechanismen (vgl. Fußnote 10, S. 71) für eine Erhöhung des Neurotransmitterspiegels im Körper sorgen.

Weitere physiologische Effekte werden im Rahmen der Verbesserung kardiovaskulärer Funktionen, der Verminderung des Spannungszustands in der Muskulatur und der zentralnervösen Aktivierung vermutet (Knobloch, 1993, S. 234).

Eine zweite Herangehensweise ist die des psychologischen Ansatzes. Er vermutet in den Bereichen der Kognitionen und des Selbstwerts die der Wirkung zugrundeliegenden Mechanismen, wobei dem Sport eher eine ‘Vehikelfunktion’ zukommt. In einer Untersuchung 
von Ossip-Klein, Doyne, Bowman, Osborn McDougall-Wilson und Neimeyer (1989) zeigen die Ergebnisse, ,... that running ... can significantly improve self-concept in clinically depressed women and that these results maintained over time“ (S. 160). Diese Beobachtungen sind auch mit Befunden von Andres (1976, S. 189 ff.) konsistent.

Ein dritter Ausgangspunkt ist das anthropologische Verständnis. Dieses betont die Verflechtung von Körper und Geist, wodurch Körpereffekte unmittelbar auch Wirkungen im Geist sind; der Mensch hat nicht nur einen Körper, er ist auch sein Leib (Knobloch, 1993, S. 234; vgl. auch die Darstellung zum Leib-Seele-Problem, S. 59 f.). Dies kommt in Körper- und Tanztherapien zum Ausdruck, wenn Gefühle durch Körperbewegungen geäußert und dadurch die Einheit von Geist und Körper wiederhergestellt werden soll.

Einige wenige Autoren betonen auch die negativen Konsequenzen des Sports, wie sie beispielsweise nach Meinung Aguglias und Sapienzas (1986) die reaktive Depression darstellt: „La depressione da successo definische le reazioni disforiche ad eventi soddisfacenti; é una depressione paradossa che segue al raggiungimento di un successo o di un vantaggio più o meno inaspettato“15 (Aguglia \& Sapienza, 1986, S. 77).

Abschließend sei noch eine eher naiv-intuitive Annäherung an den Komplex der Wirkung von Sport auf Depression erwähnt:

The concept of the body-mind relationship is ancient but only recently has psychosomatic medicine clarified how and why some functional and organic changes depend on the persistence of negative psychological conditions. Hence, it seems logical to take this phenomenon for granted in the opposite sense, that is, the persistence of positive physiological conditions, such as physical fitness ... can positively influence one's psyche. (Antonelli, 1982, S. 191 f.)

\footnotetext{
15 „Die Depression des Erfolgs bezeichnet die dysphorische Reaktion bezüglich befriedigender Ereignisse; sie ist eine paradoxe Depression, die dem Eintreten eines mehr oder weniger unerwarteten Erfolgs oder Vorteils folgt" (Übers. durch d. Verf. d. Arbeit).
} 


\subsubsection{Exkurs II: Depression als Folge der ZNS-Schädigung}

Prävalenzangaben zur Depression aufgrund einer ZNS-Schädigung differieren stark. Es konkurrieren unterschiedliche ätiologische Modelle. Eine Synthese scheint nicht möglich. Die Lateralisierung affektiver Folgen wird methodenkritisch betrachtet.

Depressionen treten häufig als Folge einer ZNS-Schädigung auf. Robinson und Price (1982) fanden nach den diagnostischen Kriterien des DSM-III-R (American Psychiatric Association, 1987) bei 106 Patienten in 46 \% der Fälle eine 'Major' oder 'Minor depression'. Insgesamt divergieren die Angaben zur Prävalenz jedoch in einem Maße, daß zuverlässige Aussagen nur schwer gemacht werden können. Herrmann (1992, S. 25) berichtete nach einer Literaturübersicht von Angaben, die zwischen 5 und $60 \%$ klinisch relevanter Depressionen nach Schlaganfall variierten. Kallert und Krebs (1995) fanden in einer Untersuchung $32.7 \%$ mittelschwere bis schwere Depressionen nach Kriterien des ICD-10 (Dilling, Mombour \& Schmidt, 1993). Diese depressiven Störungen treten nach linkshemisphärischen Schädigungen häufiger auf als nach rechtshemisphärischen. Es besteht die Tendenz, daß ,,... Läsionen der linken Hemisphäre häufiger mit dysphorischen Verstimmungszuständen, Depressionen, Angst und Katastrophenreaktionen einhergehen, während es bei Läsionen der rechten Hemisphäre eher zu Zuständen euphorischer Verstimmung oder emotionaler Indifferenz gegenüber der Läsion und deren Folgen kommt" (Wittling, 1994, S. 543). Die Hypothese, daß die linkshirnbetonte Depression lediglich eine Folge der oft mit der gleichen Hemisphärenlokalisation assoziierten Aphasie ist, konnte experimentell nicht nachgewiesen werden (Oder, Hufgard, Binder, Zeiler \& Deecke, 1991). Insgesamt scheinen affektive Störungen genauso häufig zu sein wie die stärker im Mittelpunkt der Aufmerksamkeit stehende Aphasie und Hemiplegie, doch werden sie - aufgrund der mangelnden neurologischen Relevanz - eher übersehen (Lamberti, 1993, S. 93).

Nach einer Literaturübersicht von Herrmann (1992) lassen sich drei Formen der Depression in Folge eines Schlaganfalls und anderen traumatischen ZNS-Schädigungen unterscheiden. Es handelt sich hierbei um die Konzepte der 'Grief Response', 'Catastrophic Reaction' und 'Poststroke Depression' (Herrmann, 1992, S. 28 ff.).

Die Depression kann im ersten Modell auch als Konsequenz psychosozialer Belastung verstanden werden und hat vor allem psychoanalytische Hintergründe. Die Grundannahmen besagen, daß der Betroffene mit einem dreifachen Verlusterleben konfrontiert wurde: Dem Verlust von Personen, dem des Selbst und dem von Objekten. Innerhalb dieser Konfrontation werden verschiedene Phasen durchlaufen. Zu Beginn findet eine Verleugnung im Sinne einer Abwehr statt, danach tritt die Phase der Frustration ein, in der sich der Patient der Er- 
krankungsfolgen bewußt wird. Durch dieses Bewußtwerden, das gleichsam als ein Versagen des Abwehrmechanismus gedeutet werden kann, reagiert der Patient mit einer Depression. Der weitere Verlauf dieser Depression hängt nun eng mit der folgenden Verarbeitung zusammen. Wird die Trauerarbeit durchlaufen und gelangen der Patient und Familienangehörige zu einer Haltung der Akzeptanz, weicht die Depression dieser, durch eine veränderte Sicht gekennzeichneten Grundhaltung. Dieser Prozeß muß jedoch keinesfalls zwingend als pathologisch betrachtet werden, sondern kann gemäß dieses Konzepts als Zeichen des Heilungsvorgangs und der funktionierenden Verarbeitungsprozesse aufgefaßt werden.

Der Begriff der 'Catastrophic Reaction' geht auf Goldstein (1952) zurück, der damit spezifische emotionale Reaktionsformen bei Patienten beschrieb. Diese treten besonders dann auf, wenn der Patient sich nach der ZNS-Schädigung mit Problemen konfrontiert sieht, die er, ehemals zu lösen fähig, nun nicht mehr anzugehen weiß. Bislang eingesetzte Coping-Strategien, die nach Lamberti (1994) ,... als psychische Vorgänge [gesehen werden], die darauf gerichtet sind, bereits bestehende oder erwartete krankheitsbedingte Belastungen entweder innerpsychisch (emotional/kognitiv) oder durch Handeln vorbeugend auszugleichen oder ihre Manifestationen zu verarbeiten und zu meistern“ (S. 183), greifen an diesem Punkt nicht mehr. Hierbei findet aber keine explizite Lokalisierung (im Sinne des Dualismus, vgl. S. 59) statt. Goldstein (1952) vertrat die Auffassung, daß es vergeblich ist, zwischen sogenannten organischen und funktionellen (im Sinne von konflikthaft bzw. neurotisch) Krankheiten zu unterscheiden, soweit Symptomatologie und Therapie betroffen sind (vgl. auch Lamberti, 1993). Eine Differenzierung erfuhr dieses Konzept durch Gainotti (1972), der in einer Untersuchung über die Abhängigkeit emotionaler Reaktionen von der Lokalisation einer Läsion nach Hirnschädigung verschiedene 'patterns of emotional behavior' differenzierte (nach Gainotti, 1972):

- Catastrophic Reactions: Furchtreaktionen, Tränenausbrüche, Flüche, Verweigerung einer Aussage

- Depressive Mood: Entmutigung, Wertschätzung vergangener Fähigkeiten

- Indifference Reactions: Gleichgültikeit, Anosognosie, Minimalisierung der Erkrankungsfolge

Dies stellte zugleich die Abkehr der ganzheitlich verstandenen 'Depressiven Reaktion' hin zu verschiedenen depressiven Reaktionen dar. So spiegelt sich in diesem Konzept weniger eine ganzheitliche Depressionstheorie wider, als vielmehr die Beschreibung von Verhal- 
tensweisen in konkreten Situationen, die zumeist durch Anforderungen oder bestimmte Stimuli gekennzeichnet sind.

'Poststroke Depression' ist der umfassendste Ansatz der in diesem Rahmen besprochenen. Seine Hauptaussage ist, daß anatomisch-strukturelle Veränderungen im Gehirn für die Depression verantwortlich sind. Ihm zufolge handelt es sich hierbei um endogene Depressionen, verursacht durch das Geschehen der ZNS-Verletzung per se. Eines der Modelle innerhalb dieses Ansatzes stammt von Ross und Rush (nach Herrmann, 1992, S. 36 f.). Nach ihren Vorstellungen besteht eine bilaterale zerebrale Repräsentation und Verarbeitung depressiver Verhaltens- und Erlebensweisen. Verschiedene Lokalisationen modulieren und 'triggern' diese, wobei insbesondere eine rechtstemporale Struktur, homolog dem Wernikke-Areal, an der Initiierung beteiligt ist. Durch die direkten Faserverbindungen via Corpus callosum zum Wernicke-Zentrum in der linken Hemisphäre erfahren die Gedanken eine semantisch-sprachliche Aufarbeitung und über die Verbindung zum Broca-Areal den sprachlich-motorischen Ausdruck. Analog der Verbindung des Wernicke-Areals zu dem homologen, an der Initiierung beteiligten Areal bedingt die Informationsübermittlung zu dem dem Broca-Areal homologen Areal eine affektive, sprachlich-gestische Aufarbeitung mit der Folge eines depressiven Ausdrucks.

Nach einer anderen Hypothese innerhalb der 'Poststroke Depression' stehen die Folgen eines Defizits des Neurotransmitters Monoamin im Mittelpunkt der Wirkzusammenhänge. Sie basiert auf experimentellen, psychopharmakologischen Arbeiten und der Modellvorstellung Schildkrauts (1965) und besagt, daß ,... anteriore Läsionsmuster zu einer im Vergleich zu posterioren Läsionen proximalen Unterbrechung von aus dem Tegmentum pontis aszendierenden noradrenergen oder serotonergen Neuronensystemen (als Teile des Fasciculus teencephalicus medialis) führen würden, die distal dieser Unterbrechung eine unspezifische cortikale und subcortikale Neurotransmissionsstörung zur Folge hätten“ (Herrmann, 1992, S. 37).

Ein drittes und letztes Modell innerhalb dieses Ansatzes (Swerdlow \& Kolb, 1987) macht drei unabhängige Schleifensysteme zwischen limbischem Cortex, Basalganglien, Thalamuskern und tegmentalen Mittelhirnstrukturen für die Entstehung der depressiven Störung verantwortlich. So kann eine Läsion durch die Unterbrechung von aszendierenden Fasern aus der Area tegmentalis ventralis im Bereich der tiefen Großhirnkerne alternativ Störungen im noradrenergen wie auch im serotonergen System hervorrufen oder ein striatopallidaler Faserverlust disinhibiert die cortico-thalamo-cortikale Schleife mit der Folge einer neurochemischen Dysregulation und jener Verhaltensänderung, in der sich depressive 
Muster widerspiegeln. Befunde, nach denen eine einfache Thalamusläsion nicht zur depressiven Störung führte bzw. eine Thalamotomie bereits vorhandene Depressionen lindern konnte (Herrmann, 1992, S. 38), stützen dieses Modell.

Insgesamt eher kritisch wird das Auftreten einer Depression als direkte Folge einer ZNS-Schädigung von Lamberti (1994) beurteilt. Unter der Fragestellung 'Wirklichkeit oder Artefakt?' schreibt er: „Bei insgesamt widersprüchlicher Befundlage kam die überzeugendste Kritik gegen diese Position einer neurobiologisch begründeten Depression von House et al. (1991), der zusammen mit Kollegen aus Oxford und Edinburgh die Genesung von $128 \mathrm{im}$ Durchschnitt 71 Jahre alten Personen ... verfolgte. Bei einer soziodemographisch adäquaten Kontrollgruppe $(\mathrm{N}=108)$ konnte er mittels BDI (Beck Depressions Inventar) fast gleiche Testwertverteilung nachweisen“ (Lamberti, 1994, S. 187 f.). Neben dieser inhaltlichen Kritik führt er auch eine methodische an, wonach die fehlende Einbindung von Aphasikern aufgrund fehlender visueller Analogskalen zur Depressionsbeurteilung zu einer Verzerrung führt. So können die Unterschiede in den Befunden auf verschiedene Meßmethoden zurückzuführen sein, mit der Folge, daß weder Aussagen über ein erhöhtes Depressionsrisiko nach einem Schlaganfall noch Theorien über eine diesbezügliche Ätiologie sinnvoll sind. Hinsichtlich der Lateralität eines größeren Depressionsrisikos fomulieren Herrmann, Bartels, Schumacher und Wallesch (1995), ,... critical problems in interpreting these data are posed by wide differences in the patient groups involved, time span from onset of stroke to investigation, definition of terms, measurements, and neuroradiological equipment“ (S. 850). Zerfaß, Kretzschmar und Förstel (1992), die ebenfalls die Frage der Lateralität in einer eigenen Untersuchung nicht beantworten konnten, sehen „... die Zusammenhänge zwischen Infarktlokalisation und affektiver Erkrankung [als vermutlich] ... komplizierter als in Robinsons und Starksteins ursprünglichem Modell[ $\left[{ }^{16}\right]$ angenommen ... Solange die Struktur-Funktions-Beziehungen nicht in größeren Studien mit einem verbesserten Instrumentarium überzeugend geklärt sind, bleiben Überlegungen zur Pathogenese weiterhin spekulativ“ (S. 168). Doch letztlich ist eine Methodenkritik wissenschaftlich wenig konstruktiv, als kritische Anfrage zwar sinnvoll und hilfreich, nicht aber ausreichend, die Ergebnisse ganzer Forschungsbereiche zu nivellieren.

\footnotetext{
${ }^{16}$ Eine Kernannahme besagt, daß sich die Ausprägung und Häufigkeit der affektiven Beeinträchtigung um so größer darstellt, je geringer der Abstand zwischen Infarkt und Frontalpol ist (Zerfaß, Kretzschmar \& Förstel, 1992).
} 


\subsubsection{Der depressive Realismus}

\subsubsection{Einführung in den depressiven Realismus}

Die mit Depression assoziierte Sicht der negativen Denkstile wird in Frage gestellt. Eine Untersuchung Ende der siebziger Jahre, die zu dem Ergebnis kam, daß Depressive einen größeren Realitätssinn haben, folgten viele Studien, die diesen Bfund untermauerten.

The concept of depressive realism ... has elicited reactions ranging from dismissal of the phenomenon to a fascination for it. (Dobson \& Franche, 1989, S. 419)

Sowohl von klinischen Psychologen als auch von fachkundigen Laien wurde lange Zeit davon ausgegangen, daß Depression mit pessimistischen Gedanken und negativen Denkstilen verbunden ist (Alloy, Albright, Abramson \& Dykman, 1990, S. 71; vgl. auch Kumari \& Blackburn, 1992), die als zentrale Symptomatik von Bedeutung waren und als mögliche Ursachen für die Entstehung der Depression angesehen wurden (vgl. Kap. 2.3.1.3.2, S. 73).

Ende der siebziger Jahre erregte eine Studie Aufsehen (Alloy \& Abramson, 1979), die diese Sicht in Frage stellte. In vier verschiedenen experimentellen Anordnungen standen depressive und nichtdepressive Studenten vor der Aufgabe, den Zusammenhang ('contingency' ${ }^{17}$ ) zwischen ihrer Handlung und einer experimentell gesteuerten Antwort ('outcome') zu schätzen. Vermutet wurde gemäß der Theorie der Gelernten Hilflosigkeit (vgl. Kap. 2.3.1.3.2, S. 74), daß depressive Studenten die Kontingenz unterschätzen würden. Tatsächlich zeigte sich jedoch, daß die depressiven Studenten über alle vier Variationen des Versuches hinweg 'surprisingly accurate' (Alloy \& Abramson, 1979, S. 441) waren, wohingegen sich Nichtdepressive überschätzten.

Aufgrund dieser überraschenden Wendung folgte eine Vielzahl von Studien, die versuchten, in verschiedenen Bereichen diese Befunde zu replizieren oder - gegebenenfalls zu widerlegen. Die Gründe für dieses Interesse waren vielfältig (vgl. Alloy \& Abramson, 1988, S. 223 ff.):

Zum einen stellten sie eine ,... radical departure from cognitive theory“ (Layne, 1983, S. 848) dar, durch die die Grundfeste sowohl der kognitiven depressionsätiologischen Modelle als auch der darauf basierenden Therapieformen erschüttert wurden, die auf Annahmen der

\footnotetext{
17 „Contingency is a general term and refers to the degree of relationship between any two events“ (Alloy \&
} Abramson 1979, S. 447). 
Realitätsverzerrungen seitens Depressiver als substantieller Bestandteil mitgegründet sind (vgl. auch Kap. 2.3.1.3.2, S. 73); ,... the depressive's problem is not that he or she suffers from the presence of depressogenic cognitive biases, but rather that he or she suffers from an absence of the nondepressive cognitive biases“ (Abramson \& Alloy, 1981, S. 445).

Zum anderen widersprachen diese Befunde dem allgemeinen Verständnis um psychopathologische Vorgänge, das entgegen realitätsnaher Einschätzung vielmehr Formen extremer kognitiver Prozesse, entartete Gedankenmuster oder verzerrte Wahrnehmungen bei Depressiven vermutet.

Des weiteren wird durch diese Befunde eine Verbindung zwischen der klinischen und der allgemeinen, experimentellen Psychologie hergestellt: „Historically, explanation of errors and abnormalities has led to general insights about psychological processes“ (Alloy \& Abramson, 1988, S. 224). Die Erforschung der im klinischen Bereich gefundenen Verzerrung kann nicht nur zu einem größeren Verständnis von Depression führen, sondern auch allgemeinpsychologisch die zugrundeliegenden kognitiven Prozesse beleuchten.

Eine praktische Implikation zeigen schließlich Dobson und Franche (1989) auf:

Indeed, if persons with low self-esteem and depression are actually those well anchored in reality, and the rest of the population is to some extent distorting reality in a positive direction in order to maintain a state of non-depression, then one of the criteria for mental health may have to be the maintenance of positively distorted perceptions of self and of the world. (a. a. O., S. 420)

\subsubsection{Zum Begriff der Realität}

Vor der Darstellung des depressiven Realismus muß der philosophische Realitätsbegriff und die spezifische Realität der Depressiven betrachtet werden. Der Begriff des Paradigmas wird eingeführt.

Das disziplinäre System (d. h. das Paradigma im allgemeinen Sinn) besteht aus allen Grundüberzeugungen, denen sich die Mitglieder einer wissenschaftlichen Gemeinschaft verpflichtet fühlen. Dazu gehören allgemein akzeptierte theroretische Annahmen, Analogien, Modelle und Metaphern zum gemeinsamen Forschungsgegenstand, Normen und Werte. (Westermann, 1987, S. 19; kursiv im Original)

Da der 'empirische Gehalt' (Westermann, 1987, S. 23) im Paradigma des depressiven Realismus auch auf die Realität bezogen ist, wird die Aufmerksamkeit zunächst auf den 
Begriff der Realität als die „,... Gesamtheit der materiellen Gegebenheiten, auf die sich das Verhalten des Menschen bezieht bzw. von ihm begrifflich oder in der Vorstellung erfaßt und behandelt werden kann“ (Fröhlich, 1993, S. 337 f.), gelenkt. Zwei Aspekte stechen dabei aus der Fülle der Annäherungsversuche für den Gegenstand der vorliegenden Untersuchung heraus, zum einen die philosophischen Realitätsvorstellungen, zum anderen die Aspekte der Realität, die unmittelbar mit dem Alltag der Depressiven assoziiert sind.

\subsection{Wissenschaftstheoretische Überlegungen zur Realität}

Realität wird von verschiedenen philosophischen Richtungen unterschiedlich betrachtet. Der Realismus spricht von einer von der Wahrnehmung unabhängig existierenden Realität. Objektivität kann als Intersubjektivität bezeichnet werden. Die physikalischen Reize müssen gedeutet werden, hierfür zuständige Weltanschauungen sind der Empirismus, der Rationlismus, der Idealismus und der Realismus.

For centuries, philosophers have struggled with the issue of the nature of reality.

(Dobson \& Fanche, 1989, S. 420)

Die Hauptfrage ist, ob ,... human beings have access to an objective reality, a reality that can be commonly validated by consensus" (Dobson \& Fanche, 1989, S. 421). Epistemologische Ansichten differieren stark. Ein Extrem wird vom empiristischen Standpunkt eingenommen, assoziiert mit den Thesen Lockes, Schlicks und Carnaps, demzufolge sich Wissen über die Realität in einer Akkumulation sensorischer Empfindungen erschöpft (vgl. Westermann, 1987, S. 8 ff.). Für das andere Extrem steht beispielsweise Hume, für den Kausalität fernab von Logik ein rein psychologisches Geschehen ist, das aus der Wahrnehmung von Kontiguitäten in einer tatsächlich von Willkür regierten Welt erwächst und über die in objektiver Weise keine Aussage zu machen ist - die womöglich objektiv gar nicht existiert (Dobson \& Fanche, 1989).

„The loss of the hard logical properties of reality leads to the question of whether or not an objective reality exists. These questions, which have dominated Western philosophy, have been reframed by more contemporary developmental theorists in terms of naive realism ... and relativism“ (Dobson \& Fanche, 1989, S. 421). Der Realismus spricht von einer von der Wahrnehmung unabhängig existierenden Realität, die man durch Wahrnehmung oder durch Denken erkennen kann. Im Relativismus hingegen existiert nur eine individuelle, innerpsychische Realität, ohne eine objektiv-physische Entsprechung außerhalb der Person. Objektivität ist aber in beiden Anschauungen möglich, jedoch nicht die Objektivität, welche sich als absolut versteht, sondern die der ,... Übereinstimmung der über Dinge oder Vorgänge gemachten Aussagen. Beschreiben zahlreiche Beobachter einen Sachverhalt auf 
Grund ihrer (subjektiven) Wahrnehmung in völlig gleicher Weise, so beweist dies, daß die Urteile nicht durch persönliche Eigenarten und Meinungen beeinflußt und verfälscht sind, vorausgesetzt, daß keine gegenseitige Beeinflussung unter den Beobachtern stattgefunden hat. .... Eine weniger durch metaphysische Vorstellungen vorbelastete Bezeichnung für solche Objektivität ist 'Intersubjektivität' oder 'Konkordanz' “( Traxel, 1974, S. 54 f.).

Die unterschiedlichen Sichtweisen der Realität spiegeln sich auch in den erkenntnistheoretischen Grundpositionen wider. „Ein Realist würde in einer psychologischen Theorie zumindest einen Versuch sehen, einen Aspekt der Wirklichkeit abzubilden, d. h. also beispielsweise den tatsächlichen Aufbau und die tatsächliche Funktion des menschlichen Gedächtnisses zu beschreiben. ... Idealisten dagegen würden leugnen, daß eine psychologische Theorie etwas tatsächlich Existierendes beschreibt, oder sie würden zumindest nicht annehmen wollen, daß jede Theorie als ein derartiger Beschreibungsversuch angesehen werden muß. Für Idealisten bestimmt sich der Wert einer Theorie vielmehr nach ihrem Nutzen als Instrument zur Ableitung von Vorhersagen und (praktischen) Handlungsempfehlungen“ (Westermann, 1987, S. 6 f.).

'Naive Realisten', für welche die Welt allen Menschen zumindest potentiell das gleiche Gesicht bietet, stellen den theoretischen Hintergrund für die Forschung des depressiven Realismus. Diese macht - von einer objektiven Realität ausgehend - Aussagen über eventuelle Realitätsverzerrungen seitens Personen unterschiedlicher Depressionsgrade, unabhängig von der Frage, ob dies eine der objektiven Wahrheit entsprechende Sichtweise sei: „Eine empirische Wissenschaft nimmt als Erkenntnis hin, was sich unter den einmal gegebenen Bedingungen unseres Wahrnehmens und Urteilens als Tatsache nachweisen läßt. Daß damit eine absolute, das heißt auch unabhängig von der Eigenart und den Begrenzungen menschlichen Denkens gültige Wahrheit gefunden sei, wird sie nicht behaupten“ (Traxel, 1974, S. 16). Im Sinne des Instrumentalismus, der „... Theorien als nützliche Instrumente betrachtet, die jedoch nicht die Funktion haben, Erkenntnisse über die Wirklichkeit auszudrücken“ (Gadenne, 1994, S. 313), ist diese Herangehensweise die einzig geeignete; nur so können tatsächlich Aussagen zum depressiven Realismus gemacht werden.

Die „Wahrheit“ als Leitbild der philosophischen oder wissenschaftlichen W.en [Weltanschauungen] hatte ihren Zenit im 19. Jh. Obgleich sich fast jede W. im „Besitz der absoluten Wahrheit" darstellt, kennzeichnet diese Gruppe besonders ihr Bestreben, eine Lebensausrichtung zu vertreten, die ihrer jeweiligen „Realitätsauffassung“ entspricht. Allerdings unterliegen diese Auffassungen einer Deutung. Auch der Bezug 
der „wissenschaftlichen“ W., der sich auf die physikalische Struktur der Welt und die Anpassung an die jeweils neuesten naturwissenschaftlichen Erkenntnisse stützt, enthebt nicht einer Deutung letzter Bewegunsprinzipe. Aus der Vielzahl der hierfür zuständigen W.en seien der Empirismus, der Rationalismus, der Idealismus, der Realismus sowie (als Gegenbewegung) der Nihilismus genannt. (Benesch, 1994, S. 840; kursiv im Original)

\subsection{Die Realität der Depressiven}

Nicht nur der Realitätssinn unterscheidet Depressive von Nichtdepressiven, sondern auch die individuelle Erscheinungform der Realität.

While it is undoubtedly true that depressives fail to see that "every cloud has a silver lining,' it also appears that depressives have more clouds on their horizons than do nondepressives. (Krantz, 1985, S. 607 f.)

Ein weiterer Aspekt hinsichtlich der Realität ist noch von Bedeutung: Ausgehend - zumindest im instrumentalistischen Sinne - von der Existenz einer allen gleichsam zugänglichen Realität, besteht trotzdem die Möglichkeit, daß diese für Depressive und Nichtdepressive andersgeartete Facetten bereithält.

Wenn Depressive beispielsweise von einer im Vergleich zu Nichtdepressiven geringeren Zahl an positiven Sozialkontakten berichten, kann dies einer negativen Verzerrung in der Wahrnehmung bzw. Erinnerung entspringen. Ebenso ist aber auch der Tatbestand möglich, daß Depressive, übereinstimmend mit den Befunden verschiedener Studien (vgl. auch S. 123), tatsächlich weniger soziale Anerkennung genießen; ,... unhappy, hopeless thoughts are not irrational if the situation actually is aversive or hopeless" (Layne, 1983, S. 848). Bei einer Untersuchung in einem sozialen Kontext wurde deutlich: „In support of depressed persons' responsivity to situational information, we found that independently of their more negative schemata, the depressed subjects more negative social performance in the group discussion contributed significantly to their more negative interpretation of ambiguous feedback“ (Dykman, Horowitz, Abramson \& Usher, 1991, S. 53).

So haben die Nichtdepressiven vielleicht gerade aufgrund ihres realitätsverzerrenden Optimismus mehr Erfolg; er rüstet sie mit der nötigen Zuversicht aus, im Kampf gegen die Widrigkeiten des Alltags zu bestehen. Die Depressiven hingegen, die „do, in fact, live more difficult lives than do nondepressives ...“ (Layne, 1983, S. 848), werden durch ihre Hoff- 
nungslosigkeit - unterstützt durch Mechanismen wie die der 'self-fulfilling prophecy' (vgl. Beck, Rush, Shaw \& Emery, 1994, S. 294; vgl. auch S. 181) - in ihrer alltäglich notwendigen Beharrlichkeit geschwächt und haben somit als Folge tatsächlich weniger Erfolgserlebnisse. Daraus entsteht ein sich selbst verstärkender Kreislauf (vgl. Lewinsohns Verstärkermodell, S. 79), der die Depressiven immer tiefer - in dem Maße, in dem die Depression durch die Umweltfaktoren aufrechterhalten wird - in die Depression versinken läßt.

\subsubsection{Forschungsbereiche der Realismushypothese: Befunde und Im- plikationen}

Die Realismushypothese ist auf verschiedenen Gebieten erforscht worden. Die untersuchten Richtungen lassen sich in die Bereiche der Kontingenzschätzung, der Erwartung und Vorhersage von Ereignissen, Attributionsmustern, Rückmeldungen und der Selbstbewertung einteilen.

Im folgenden werden die Befunde, die als Hinweise für die Evidenz des depressiven Realismus zu verstehen sind, innerhalb der fünf großen Bereiche, in denen die Hypothese des depressiven Realismus untersucht wurde, dargestellt. Verschiedene Implikationen für Theorie und Alltag werden angeführt. Der Überblick beginnt zunächst mit Studien, die im Bereich der Kontingenzschätzung (S. 93 ff.) das Phänomen einer größeren Realitätsnähe auf seiten der Depressiven entdeckten. Andere Untersuchungen beschäftigten sich - hinsichtlich der Unterschiedlichkeit depressiver und nichtdepressiver Personen - mit Erwartung und Vorhersage von Ereignissen (S. 105 ff.), der Unterschiedlichkeit in den Attributionsmustern (S. 112 ff.) und, neben dem Umgang mit Rückmeldungen (S. 116 ff.), mit der Selbstbewertung (S. 121 ff.). Das Ziel dieser Darstellung besteht darin, einen Einblick zu vermitteln, wie vielfältig der depressive Realismus untersucht und bestätigt wurde, um im Anschluß hieran die vorliegende Untersuchung besser in den Gesamtkontext der Forschung integrieren zu können. Zum Teil stehen die referierten Studien recht unverbunden nebeneinander. Dies entspricht jedoch der Intention dieses Kapitels, nicht reduktionistisch, sondern der Vielfalt angemessen, nicht interpretierend, sondern darstellend vorzugehen. 


\subsection{Einschätzung der Kontingenz}

Der Einschätzung des Kontingenzgrades zwischen der eigenen Handlung und einer experimentell gesteuerten Konsequenz gehört zu dem größten und wichtigsten Bereich, in dem die Realismushypothese getestet wurde.

Untersuchungen, in denen Personen den Grad der Kontingenz zwischen der eigenen Handlung und einer experimentell gesteuerten Konsequenz ('outcome') schätzen müssen, gehören nicht nur zu dem größten und wichtigsten Bereich, in dem die Realismushypothese getestet wurde, mit ihnen begann auch die Geschichte der 'Sadder but wiser'-Forschung. Die Wichtigkeit dieses Bereichs wird des weiteren durch den spezifischen Umstand gestützt, daß die Realität, die tatsächliche Kontingenz, dank problemadaptierter Computerprogrammierung objektiv erfaßt und exakt ermittelt werden kann. Darüber hinaus ist von grundsätzlicherer Bedeutung, daß ,... the ability to detect the relationships or covariations among stimuli, behavior, and outcomes in one's environment is an important component of adaptive behavior“"(Alloy \& Tabachnik, 1984, S. 112).

Den Grundstein für diese Forschung legten Alloy und Abramson (1979) mit einer Studie, deren abschließende Frage im Titel - „,... sadder but wiser?“ (Alloy \& Abramson, 1979, S. 441) - in der Folgezeit oft bemüht wurde, wenn es galt, die hier behandelte Hypothese prägnant zusammenzufassen. Die übergreifende Fragestellung war, wie sich die durch eine Person wahrgenommene Kontingenz gegenüber der jeweils wirklichen Kontingenz verhält, denn ,the concept of contingency provides a cornerstone for a number of highly influential contemporary theories of learning“ (a. a. O., S. 442).

In jedem der vier von Alloy und Abramson (1979) durchgeführten Experimente galt es, Kontingenzen zu lernen. Konkret handelte es sich um das Lernen eines Zusammenhangs zwischen einer fakultativen Aktion und einer experimentellen Konsequenz. Jedes der vier Experimente bestand aus 40 Versuchen, die jeweils 3 Sekunden dauerten und durch ein gelbes Licht eingeleitet wurden. Nach Aufleuchten dieses Lichts konnten die Versuchspersonen innerhalb der vorgegebenen Zeit einen Knopf drücken. Anschließend leuchtete nach einem durch die Versuchsleiter festgelegten Verhältnis zwischen dem Drücken bzw. dem Nichtdrücken und der experimentellen Konsequenz das grüne Licht (welches das 'outcome' darstellte) auf.

Die Resultate zeigten, daß ,... people do have a concept of contingency, that in the response-outcome case entails the relative efficacy of one response versus another in determining outcomes“ (a. a. O., S. 474). Auf die Empirie bezogen konnte dies jedoch in einer solchen Eindeutigkeit nur für den ersten Versuch gesagt werden; die über einen großen Bereich der Versuchsvariationen realitätsangepaßte Einschätzung verlor diesen Realitätsbezug 
in einer systematischen Weise in den restlichen drei Experimenten. In dem Gruppenvergleich von Depressiven ${ }^{18}$ und Nichtdepressiven zeigte sich sowohl bei einem tatsächlich unkontrollierbaren Ergebnis mit einem häufigen Aufleuchten des Lichts (Experiment 2) und bei einer gesteigerten Attraktivität des Ergebnisses (Geldgewinn; Experiment 3), daß Nichtdepressive ihr Ausmaß an Kontrolle überschätzten bzw. unterschätzten, als es sich um Geldverlust handelte (Experiment 4). Die depressiven Studenten schätzten unabhängig von der jeweiligen Größe der tatsächlichen Kontingenz diese über alle Bedingungen relativ genau. Insgesamt schien es sich bei der Fehleinschätzung der Nichtdepressiven aber nicht um Schwierigkeiten im Wahrnehmungsprozeß zu handeln, da über alle vier Versuche sowohl die depressiven als auch die nichtdepressiven Studenten die bedingten Wahrscheinlichkeiten für das Aufleuchten des grünen Lichts akkurat wahrnahmen; die Problematik erwuchs aus der Organisation der Informationen, die zum Schätzen der Kontingenz rekrutiert wurden (Alloy \& Abramson, 1988, S. 229).

Mit der für die Überprüfung der Theorie der 'Gelernten Hilflosigkeit' schon fast charakteristischen Versuchsanordnung ${ }^{19}$, testeten Alloy und Abramson (1982) einige Jahre später Personen vor dem Hintergrund des Phänomens der illusionären Kontrollüberzeugung ("illusion of control ${ }^{20}$ ). Hierbei erschien die experimentelle Konsequenz (ebenfalls das Aufleuchten eines grünen Lichts) immer ohne Bezug zu der Handlung der Versuchsperson und war, in Abhängigkeit von der experimentellen Variation, entweder mit Gewinn oder Verlust von Geld verbunden.

Auch in dieser Untersuchung schätzten Depressive, unabhängig von der Vorbehandlung, und Nichtdepressive, mit kontrollierbarem Lärm in der Vorbehandlung, in beiden Versuchsbedingungen akkurat ein, daß sie keine Kontrolle über das Aufleuchten des Lichts hatten. Die Nichtdepressiven jedoch, die vorab 'Hilflosigkeit gelernt' hatten, zeigten in der Gewinnsituation illusionäre Kontrolle. Für die Theorie der Gelernten Hilflosigkeit sind diese Befunde keine Untermauerung, da nach dieser depressive und 'gelernt hilflose' Personen die Tendenz haben, ihren Einfluß auf Situationen zu unterschätzen, in der Versuchsanordnung beispielsweise Kontingenzen geringer als tatsächlich vorhanden wahrzunehmen

\footnotetext{
${ }^{18}$ Als depressiv wurden Studenten klassifiziert, die einen BDI-Wert (vgl. S. 145) von 9 oder mehr hatten (Alloy \& Abramson, 1979, S. 449).

${ }^{19}$ Diese Versuchsanordnung sieht drei Gruppen vor; die erste wird kontrollierbarem, die zweite nichtkontrollierbarem und die dritte keinem Lärm ausgesetzt.

${ }^{20}$ Nach Langer (1975) ist dieser Ausdruck definiert als ,... expectancy of a personal success probability inappropriately higher than the objective probability would warrant" (Langer, 1975, S. 313).
} 
(Alloy \& Abramson, 1982, S. 1122). Weit wichtiger scheint aber eine andere Implikation; durch das unterschiedliche Verhalten der Depressiven und der Nichtdepressiven, die aber der Hilflosigkeitssituation ausgesetzt waren, zeigte sich die grundlegende Verschiedenheit dieser beiden Gruppen. Damit ist die Basis vieler Experimente, die 'Depression' über das Paradigma der Gelernten Hilflosigkeit induzieren, zumindest in Frage gestellt.

'Vorhersagbarkeit'21 ist der Parameter, der bei Alloy, Abramson und Kossman im Jahre 1985 in den Mittelpunkt des Interesses gerückt wurde. Das erste Versuchsereignis birgt in sich Informationen, die über die Wahrscheinlichkeit des Stattfindens des zweiten Ereignisses Auskunft geben; je nach Versuchsanordnung ist der Informationsgrad stärker oder schwächer ausgeprägt, oder aber, als dritte Variation, fehlt die Information ganz.

Auch wenn die Autoren betonen, daß es sich bei Depression nicht um ein einheitliches Krankheitsbild handelt (Alloy, Abramson \& Kossman, 1985, S. 232), und Abramson selbst an der Entwicklung eines eventuellen Subtyps, der 'hopelessness depression', beteiligt war (vgl. Abramson, Metalsky \& Alloy, 1989; vgl. auch S. 75), bezieht sich die Forschung unspezifisch auf alle Formen der Depression. Für eine Differenzierung einzelner Depressionstypen stand die Wissenschaft zum Zeitpunkt der Studie noch zu sehr am Anfang. Sie würde aber einen Ausweg darstellen, die Theorie der Gelernten Hilflosigkeit aus einer Lage zu befreien, in der die Ableitungen, die aus dieser Theorie vorgenommen werden können, nicht mit der beobachtbaren Realität übereinstimmen. Nach ihrer Vorhersage haben die Depressiven gelernt, daß zwischen ihrem Tun und der Reaktion nur sehr wenig Zusammenhang besteht, so daß sie in einem Experiment, in dem es Kontingenzen zu schätzen gilt, die tatsächliche Kontingenz unterschätzen müßten. Da dies für Depressive vielfach nicht zutrifft, wäre es denkbar, daß die Depressiven, die untersucht wurden, nicht dem Subtyp der gelernten Hilf- oder Hoffnungslosigkeitsdepression zuzuordnen wären.

$\mathrm{Ob}$ die Nichtdepressiven in gleichem Maße einer 'illusion of predictability' wie der 'illusion of control' verfallen, ist die Frage, die dieser Untersuchung vorangestellt wurde (Abramson \& Kossman, 1985, S. 234). Das experimentelle Vorgehen glich der ersten Studie (Alloy \& Abramson, 1979; vgl. S. 94) mit dem Unterschied, daß nun lediglich der Grad der Vorhersagbarkeit des grünen Lichts aus dem Aufleuchten eines roten Lichts abzuschätzen war. Zusätzlich war das Aufleuchten des grünen Lichts in dem ersten der drei Versuche negativ mit der aktuell vorhandenen Kontingenz korreliert, so daß die Einschätzung einer

\footnotetext{
${ }^{21}$ Während hier - innerhalb der Verbindung zweier Ereignisse - mit 'Kontrolle' die Abhängigkeit des zweiten Ereignisses von der Aktion einer Person zwischen den Ereignissen gemeint ist, handelt es sich bei gleicher Versuchsanordnung ohne diese Abhängigkeit um 'Vorhersagbarkeit'.
} 
hohen Vorhersagbarkeit, schon aufgrund der Häufigkeit des Aufleuchtens des grünen Lichts, weniger genau war. In diesem Versuch zeigte sich jedoch eine hohe Genauigkeit in der Einschätzung, sowohl auf seiten der Depressiven als auch auf der der Nichtdepressiven. Ein Vergleich mit der Studie von 1979 (ebd.) zeigte sogar, daß die Kontingenz unter der Bedingung, daß das Ausbleiben des roten Lichts mit dem Aufleuchten des grünen Lichts verbunden war, ähnlich genau eingeschätzt wurde wie in der Situation, in der das Aufleuchten des roten Lichts mit der höheren Rate an Verstärkung (grünes Licht nach Knopfdruck) assoziiert war.

Das Erkennen von Nichtkontingenz war die Aufgabe der zweiten und dritten Untersuchung dieser Studie. Zuerst galt es herauszufinden, was geschieht, wenn das Ergebnis nicht mehr neutral ist, sondern eine positive oder negative Konnotation (Geldgewinn bzw. verlust) erhält. Die Nichtdepressiven zeigten sich, im Gegensatz zu der Studie von Alloy und Abramson (1979), genauso realitätsnahe wie die Depressiven (S. 237). Die Autoren folgerten für den Fall, in dem nicht die eigene Antwort der jeweiligen Person gefordert ist, daß die Nichtdepressiven keinem illusionären Vorhersagbarkeitsglauben verfallen.

In der letzten experimentellen Anordnung ging es schließlich um den Vergleich von Vorhersage und Kontrolle, wenn das experimentelle 'outcome' hochfrequent positiv (das grüne Licht erschien sehr oft) war, ohne daß jedoch ein Zusammenhang mit der Handlung der Versuchsperson bestand. Tatsächlich war bei den Nichtdepressiven die Tendenz zu beobachten, daß sie in der Kontrollsituation die Kontingenz höher einschätzten als in der Vorhersagebedingung, wobei die tatsächliche Kontingenz in beiden Bedingungen identisch war. Im Vergleich der Geschlechter war diese Tendenz bei weiblichen Versuchspersonen noch stärker ausgeprägt als bei den männlichen. Die Depressiven gaben realitätsnah über beide Versuchsbedingungen hinweg geringe Kontingenzen an (Alloy \& Abramson, 1985).

Zusammen mit Viscusi untersuchten Alloy und Abramson ,... the directional of the relationship between realism ... in judging personal control and depressed affects“ (Alloy, Abramson \& Viscusi, 1981, S. 1130). Zum Zeitpunkt der Studie war die Kausalität des Zusammenhangs zwischen Depression und der Genauigkeit der Einschätzung nicht bekannt. Den Verfassern zufolge stehen drei Alternativen zur Disposition: Die Depression kann die Ursache sein, daß Menschen ihre Wirkung auf Ereignisse realistischer einschätzen, Personen, die ihre Wirkung realistischer wahrnehmen, sind anfälliger, depressiv zu werden oder eine mögliche Drittvariable kann identifiziert werden, die sowohl zur Depression als auch zur besagten Genauigkeit führt (ebd.). 
Experimentell wurde dieser Fragestellung mit dem Vorgehen im 'Experiment 3' (Alloy \& Abramson, 1979, S. 462 ff.; vgl. auch S. 94) nachgegangen (Fehlen von Kontingenz und Assoziation des experimentellen Ergebnisses ('outcome') mit Geldgewinn). Um die eingangs erwähnte Frage bezüglich der Kausalität zumindest in Teilen beantworten zu können, wurde weiblichen Depressiven und Nichtdepressiven eine ihrer normalen Verfassung gegenläufige Stimmung induziert: Die Depressiven wurden experimentell aufgeheitert, die Nichtdepressiven wurden in eine eher niedergeschlagene, traurige Stimmung versetzt. Unter der Annahme, daß die induzierte Stimmung die Exaktheit der Einschätzung des Ausmaßes persönlicher Kontrolle beeinflußt, hätten die in Hochstimmung versetzten Depressiven die Tendenz aufweisen müssen, das Ausmaß der Kontrolle zu überschätzen, während die ehemals Nichtdepressiven in ihrem Urteil ein größeres Maß an Genauigkeit hätten beweisen müssen. Tatsächlich entsprachen die Ergebnisse dieser Vermutung (Alloy, Abramson \& Viscusi, 1981, S. 1135 ff.). Der Vergleich mit einer Gruppe, die die ihnen gegenläufige Stimmung lediglich simulieren sollte, zeigte, daß die Inversion nicht der eventuell unbewußten Absicht der Teilnehmer entsprang, in ihrem Handeln den Versuchsanforderungen gerecht zu werden. Zusätzlich konnten durch weitere Kontrollgruppen, denen keine oder neutrale Stimmungen induziert wurden, auch diejenigen Ergebnisse repliziert werden, zu denen die erste Studie kam (Alloy \& Abramson, 1979, S. 464 ff.; siehe auch S. 94). In dem Urteil über die Präzision ihrer eigenen Einschätzung unterschieden sich die einzelnen Gruppen nicht (a. a. O., S.1137).

Ungeklärt bleibt allerdings die Frage, durch welche Mechanismen eine aktuelle Stimmung den Kontrollglauben beeinflussen kann, da sich die Forschung bislang intensiver mit den kognitiven Prozessen bei Depressionen beschäftigt hatte und sich weniger um eine detaillierte Beschreibung von Stimmungen bemühte. Im Grunde beinhaltet die Fragestellung dieser Studie nur einen Teilaspekt der übergeordneten Überlegung, welche kognitiven Prozesse die Folge und welche die Ursache von bzw. für Depression sind (a. a. O., S. 1138).

Mit dem Versuchsdesign des zweiten Experiments von Alloy und Abramson (1979, S. 457 ff.; vgl. auch S. 94) näherten sich Benassi und Mahler (1985) dem Komplex des ‘judgment of contingency’ an. Ihr Interesse galt der Überlegung, ob ,... judgments of personal control in contingency learning experiments are influenced by the private/public variable“ (Benassi \& Mahler, 1985, S. 1324). Die meisten experimentellen Anordnungen isolieren die Versuchsperson, während im Alltag das Abschätzen der eigenen Kontrolle auch im sozialen Kontext geschieht. Angeregt wurde die Überlegung durch Autoren wie Sacco und Hokanson (1982), nach deren Urteil in den Arbeiten Alloys keine theoretischen oder 
empirischen Hinweise auf einen solchen Einfluß bestanden. Lediglich Langer (1975) vermutete, daß die Anwesenheit einer anderen Person den Anspruch an Rationalität derart erhöhe, daß Nichtdepressive die eigene Kontrolle vergleichsweise geringfügiger überschätzen würden.

Im ersten Experiment wurde die gesamte Aufgabe in Anwesenheit eines Beobachters durchgeführt, mit der Folge, daß nun die Depressiven den Grad ihrer Kontrolle überschätzten, während die Beurteilung der Nichtdepressiven exakter wurde. Damit waren die Befunde genau invers zu den sonstigen Trends, die in diesem Kontext beobachtet wurden (vgl. Alloy und Abramson, 1979, S. 457 ff.; S. 94) und die auch in der 'privat'-Bedingung dieses Experiments repliziert werden konnten (Benassi \& Mahler, 1985). Im zweiten Experiment wurde untersucht, in welchem Ausmaß die Häufigkeit ('frequency') des experimentellen 'outcome' einen Einfluß auf die Kontrolleinschätzung hat. Dabei wurde deutlich, daß die Replikation der Befunde des ersten Experiments nur für die Bedingung der hohen Frequenz möglich war. Bei der geringeren Frequenz unterschieden sich die beiden Gruppen nicht (Benassi \& Mahler, 1985, S. 1326). Das dritte Experiment bewies, daß die Anwesenheit eines Beobachters in der Phase, in der die Kontingenzen 'gelernt' werden, ausreicht, um die Depressiven im Vergleich zu den Nichtdepressiven zu höheren Kontrolleinschätzungen zu verleiten. Der Beobachter verließ den Raum, nachdem die Lernphase beendet war, aber noch bevor der Fragebogen für die Einschätzung der Kontrolle ausgefüllt wurde (a. a. O., S. 1327 f.). So zeigt sich bereits hier eine Einschränkung des Geltungsbereichs der 'Sadder but wiser'-Hypothese, die auch den Depressiven unter den beschriebenen Bedingungen eine Verzerrung ('bias') nachweist.

Eine Ausweitung des Forschungsbereichs nahmen Martin, Abramson und Alloy (1984) vor, indem sie nicht mehr nur die Urteile über das Ausmaß der eigenen Kontrolle erhoben, sondern auch untersuchten, inwieweit Depressive oder Nichtdepressive glauben, daß eine andere Person Kontrolle habe; „... researchers of cognitive processes and depression generally have ignored depressives' perceptions and cognitions about other people“ (Martin, Abramson \& Alloy, 1984, S. 126). Insgesamt ist aber gerade die Unterscheidung von Selbstsicht und Fremdsicht bei Depressiven ein neuralgischer Punkt. Sowohl speziell nach der Depressionstheorie von Beck (Beck, Rush, Shaw \& Emery, 1994), als auch allgemeiner nach Ansätzen der Sozialpsychologie (Frey \& Greif, 1987) entspringt die Selbstbewertung dem Vergleich des Selbst mit dem anderer Personen, so daß „,... social comparison processes can be used to enhance oneself ...“ (Taylor, 1983, S. 1166). Das geringer ausgeprägte Selbstwertgefühl, das bei Depressiven zu beobachten ist, resultiert eventuell aus ei- 
ner für die depressive Person negativen Art dieses Vergleichs. Tabachnik, Crocker und Alloy (1983) berichteten von einem Zusammenhang zwischen dem Grad der Depression und der Tendenz, den eigenen Wert abzumindern, daß ,within the depressed group, the more depressed subjects were, the more DR [depressionsrelevante] items they rated as truer of themselves than of the average college student ..." (Tabachnik, Crocker \& Alloy, 1983, S. 693). Im Hinblick auf das Alltagsleben entbehrt dieser Umstand nicht einer gewissen Tragik, da sich bei niedrigem Selbstwertgefühl die Wirkung von negativen Erlebnissen noch verstärkt; in einer Untersuchung von Campell und Fairey (1985) präsentierten sich Personen mit geringem Selbstwertgefühl in Anagrammaufgaben, nachdem sie einen hypothetisch antizipierten Mißerfolg in der Aufgabe erklären mußten, schlechter als diejenigen mit hohem Selbstwertgefühl, während nach der Erklärung, wie es zu einem ebenfalls hypothetisch antizipierten Erfolg kam, kein Unterschied zwischen den Gruppen festzustellen war (S. $1101 \mathrm{ff}$.).

Aus diesen Gedanken leitet sich die Vermutung ab, daß Depressive in ihrer Sicht für andere der illusionären Kontrollüberzeugung anheim fallen und diese ,... as exerting a large impact on the green light“" (Martin, Abramson \& Alloy, 1984, S. 127) wahrnehmen; zur experimentellen Überprüfung wurde diesmal das Design des dritten Experiments von Alloy und Abramson (1979, S. 462 ff.) bemüht (Abwesenheit von Kontingenz, Verbindung des grünen Lichts mit Geldgewinn). Die Erweiterung bestand darin, daß jede Person einen Verbündeten hatte, für den sie zusätzlich zu der eigenen Kontrolleinschätzung ein Urteil bezüglich dessen Ausmaß an Kontrollvermögen abgeben mußte. Von den Nichtdepressiven wurde erwartet, daß sie für sich selbst zuviel Kontrolle in Anspruch nehmen, aber, indem sie sich diesbezüglich höher als den gemeinen Durchschnitt einschätzen (Martin, Abramson \& Alloy, 1984, S. 127), die Kontrolle anderer realistischer beurteilen. Da es in der Literatur Hinweise darauf gibt, daß das Geschlecht der zu beurteilenden Person in derartigen Situationen eine wichtige Rolle spielen kann, wurde diese Variation in den Versuchsplan mit aufgenommen.

Während unter der Bedingung der Einschätzung eigener Kontrollüberzeugungen die Ergebnisse der 'Sadder but wiser'-Forschung repliziert wurden, zeigten die Depressiven in ihrem Urteil über das Ausmaß der Kontrolle anderer eine zu hohe Einschätzung; sie verfielen der 'illusion of control'. Als einzige Ausnahme erwies sich diejenige Kombination, in der männliche Depressive für männliche Verbündete urteilten, so daß insgesamt zumindest die weiblichen Teilnehmer mit den Vorhersagen konsistent handelten (Martin, Abramson \& Alloy, 1984, S. 133). Aus diesen Ergebnissen wird deutlich, daß die Exaktheit des Einschätzungsvermögens der Depressiven, die ihnen in diesem Forschungskomplex immerhin 
das Attribut 'wiser' einbrachte, weder von einem ausgeprägteren Geschick im Umgang mit Problemlösestrategien abzuleiten ist, noch aus einer größeren Fähigkeit, Kontingenzen zu schätzen (ebd.). Insgesamt bestätigen diese Ergebnisse die Wichtigkeit, interpersonale Wahrnehmung in die Depressionsforschung zu integrieren.

Nach Vázquez (1987) ist das Vorgehen von Alloy und Abramson (1979) möglicherweise mit einem konzeptionellen Problem behaftet; sie benutzten mit dem Licht (das grüne Licht, dessen Aufleuchten das experimentelle 'outcome' darstellt) einen sehr unspezifischen Reiz. Ihm zufolge hatte aber gerade die zu diesem Zeitpunkt neuere Depressionsforschung die Wichtigkeit von verbalen Stimuli, die depressionsspezifische Aspekte beinhalten, aufgezeigt (Vázquez, 1987, S. 420).

In vier Experimenten arbeitete er zweimal mit neutralen und zweimal mit verbalen Stimuli, wobei sich diese Paare noch einmal in jeweils eine Bedingung mit und eine ohne Kontingenz aufteilten. In den ersten beiden Experimenten (Design von Alloy \& Abramson, 1979, Experiment $1+2$; vgl. S. 94), erhielt er ähnliche Ergebnisse wie Alloy und Abramson im Jahr 1979. Vázquez untersuchte Studenten der Universität von Madrid. Nach seiner Hypothese sollten die Depressiven Kontingenzen dann höher einschätzen als es der aktuellen Lage entsprach, wenn die auf sie bezogenen Sätze negativ waren, bei den Nichtdepressiven sollte dies in der Bedingung der positiven Sätze eintreten (Vázquez, 1987, S. 424).

Das Ergebnis zeigte, daß „,... nondepressed subjects evaluated accurately the actual degree of contingency in the negative condition and overestimated in the positive condition. On the other hand, depressed subjects judged the degree of contingency accurately in the positive conditions but, contrary to predictions, did not overestimate the actual degree of contingency when negative sentences were presented“ (Vázquez, 1987, S. 425). Dem vierten Experiment wurden noch einmal die Erwartungen des dritten Experiments zugrundegelegt, die Variation bestand in der Abwesenheit von Kontingenz und einer Erhöhung der Frequenz der experimentellen Reaktion. Unter diesen Umständen zeigten sich die Erwartungen als vollständig konsistent mit der experimentellen Realität. Nichtdepressive und Depressive bewiesen ihre Anfälligkeit für das Phänomen der illusionären Kontrolle; die Depressiven, wenn die Sätze einen negativen Inhalt hatten, die Nichtdepressiven analog bei den positiven Sätzen. Exaktheit wurde in der jeweils anderen Bedingung von beiden Gruppen bewiesen.

Deutlicher war die Befundlage wiederum bei Ford und Neale (1985). In der klassischen Vorgehensweise der Experimente im Rahmen der Theorie der Gelernten Hilflosigkeit (vgl. Kap. 2.3.1.3.2, S. 74) wurde einer Gruppe von Studenten der Psychologie Hilflosigkeit in- 
duziert, eine andere Gruppe diente als Kontrollgruppe. Da es sich bei den motivationalen und kognitiven Defiziten bei 'gelernt hilflosen' Personen um Ecksteine der besagten Theorie handelt (Ford \& Neale, 1985, S. 1330) und diese eine Auswirkung auf die Güte einer auszuführenden Aufgabe haben, in der Kontrolle möglich ist, wurde erwartet, daß mit dem Untersuchungsdesign von Alloy und Abramson (1979; vgl. S. 94) die Versuchspersonen der Experimentalgruppe die tatsächlichen Kontingenzen unterschätzen würden. Entgegen diesen Erwartungen war die Kontrolleinschätzung der Experimentalgruppe sogar höher als die der Kontrollgruppe, so daß im Betrachten der Durchschnittswerte nicht nur der aus der besagten Theorie resultierende Trend umgekehrt wurde (a.a. O., S. 1335), sondern die Nichthilflosen auf den ersten Blick in ihrer Einschätzung auch als exakter gelten konnten, wohingegen sich die Induktionsgruppe scheinbar überschätzte. Auf den zweiten Blick jedoch, unterstützt durch eine statistische Auswertung der Abweichungen, zeigt sich die gröBere Genauigkeit auf seiten der 'gelernt Hilflosen': „,... subjects in the induction group made reliably higher and more accurate judgments of control than subjects in the notreatment group ... the direction ... is especially compelling evidence in contradiction of helplessness theory ..." (Ford \& Neale, 1985, S. 1335). Inwieweit die Befunde auf eine reduzierte Fähigkeit der Depressiven zurückgehen, komplexere Hypothesen zu gebrauchen (Alloy \& Abramson, 1979), ist durch die Studie nicht beantwortet. Die Operationalisierung einer größeren Komplexität bestand darin, in dem Aufleuchten des Lichts oder in der eigenen Antwort (respektive einer Kombination von beidem) ein bestimmtes Muster zu suchen. Bezogen auf die beiden Gruppen konnten keine Unterschiede festgestellt werden, was nach Meinung der Autoren jedoch auch auf konzeptionelle Probleme im Design dieser Teilerhebung begründet liegen kann (Ford \& Neale, 1985, S. 1335). Des weiteren ist die Auswirkung der Motivation genausowenig geklärt wie die Implikation der Hypothese, daß Erfahrungen mit Nichtkontingenzen eine Erwartung der Zusammenhangslosigkeit von kommenden Ereignissen konstituieren. Ohne ein klares Erfassen der Motivation kann in Experimenten dieser Art bei Abnahme der Leistung nicht von einer Auswirkung des Motivationsschwunds gesprochen werden, da auch Befunde denkbar sind, denenzufolge eine hohe Motivation genauso hinderlich ist wie die reduzierte Motivation, daß also in einem Mittelbereich des Motivationsgrades die besten Ergebnisse zu erwarten sind. Diese Umkehrung des gerichteten Trends in eine U-Funktion wird von Ford und Neale (1985) unter Hinweis auf einschlägige Befunde (a. a. O., S. 1336) zumindest als Möglichkeit diskutiert. Eine weitere ist das Phänomen der Reaktanz (vgl. Weiner, 1988; vgl. auch S. 180), innerhalb derer Personen durch das Erfahren einer Nichtkontrolle motivierter sind, in einer nächsten Situation 
Kontrolle auszuüben. Ähnlich verhält es sich mit der Erwartung bezüglich Kontingenz. Dadurch, daß nicht die Erwartung selbst erhoben wird, sondern aus den experimentellen Ergebnissen indirekt Rückschlüsse gezogen werden, handelt es sich um eine zirkuläre Logik, die keine Auskunft über zugrundeliegende Abhängigkeiten geben kann (Ford \& Neale, 1985, S. 1335).

Als eines der Begleitphänome von Depression gilt nach Alloy und Abramson (1979) das motivationsgesteigerte Defizit, komplexe Hypothesen zu erstellen. Das Experiment dieser Autoren hingegen erforderte lediglich einfache Hypothesen, um Erfolg zu haben. Es genügte, sich häufiger für einen Knopfdruck zu entscheiden, um die versuchsimmanente Kontingenz in hohem Maße erfahren zu können. Somit kann die größere Genauigkeit seitens der depressiven Personen auch eine Folge des Umstands sein, daß im Versuchsdesign explizit die motivationalen Defizite von den kognitiven isoliert wurden. Diese beiden Defizite resultieren nach der Theorie der Gelernten Hilflosigkeit aus der Überzeugung depressiver Personen, daß ihre Handlungen in der sozialen und materiellen Umwelt keine oder wenig Wirkung haben (Abramson, Alloy \& Rosoff, 1981). Hierbei verhindern die kognitiven Folgen die Wahrnehmung von Kontingenzen, während die motivationalen Einschränkungen eine ,... retarded initiation of voluntary responses “ (Abramson, Alloy \& Rosoff, 1981, S. 35) bewirken.

Nachdem die rationale Komponente in der Studie von 1979 bereits im Mittelpunkt stand, untersuchten Abramson, Alloy und Rosoff (1981) in Verbindung mit den schon angesprochenen komplexen Hypothesen stärker die motivationale Seite: „The logic here is that if depressives exhibit the motivational deficits of helplessness, they will be less likely to generate the appropriate hypotheses and therefore will not be exposed to the potential contingency between their responses and the outcome“ (Abramson, Alloy \& Rosoff, 1981, S. 37). Sind Depressive dann, wenn sie die Hypothesen nicht selbst entwickeln müssen, in der Lage, Kontingenzen realitätsgerecht einzuschätzen, scheitern jedoch in der Bedingung, in der sie selbst diese Annahmen generieren müssen, beweisen sie die motivationalen Defizite; sind sie in keiner der beiden Bedingungen dazu in der Lage, handelt es sich bei ihnen auch um Mängel kognitiver Art. In der Methodik orientierte sich diese Studie ebenfalls an dem äußeren Rahmen der Untersuchung von 1979 (Alloy \& Abramson, 1979; siehe S. 94).

Das experimentelle Resultat ist bei diesem Design potentiell kontrollierbar (Verstärkungsrate von $75 \%$ ), die Handlung ist physisch sehr leicht auszuführen; kognitiv existiert jedoch die Schwierigkeit, nur mit einer komplexen Hypothese (der Knopf ist in ei- 
nem wohldefinierten Zeitintervall innerhalb der 5 Sekunden zu drücken ${ }^{22}$ ) den erwähnten Grad der Kontrolle tatsächlich zu erfahren. Die Einschätzung der Versuchspersonen hinsichtlich des Kontrollgrades war die wichtigste abhängige Variable, und deren Werte stellten auch den bedeutsamsten Teil der Ergebnisse dar: Die Nichtdepressiven schätzten realitätsnah ihr Ausmaß an Kontrolle auf etwa $75 \%$, und dies sowohl für den Fall, daß die komplexen Hypothesen selber entwickelt werden mußten als auch für den Fall, daß die richtige Hypothese in einem kleinen Pool von möglichen Hypothesen vorgegeben war. Depressive Personen zeigten einen vergleichbaren Grad an Genauigkeit nur dann, wenn sie nicht selbst die Hypothese erstellen mußten; unter der anderen Modalität gaben sie deutlich weniger Kontrolle an (Abramson, Alloy \& Rosoff, 1981, S. 40 f.). Somit sind die Ergebnisse hypothesenkonform, wie auch der Befund, daß die Depressiven das potentielle Ausmaß der Kontrolle unterschätzten, wenn sie die Hypothesen selbst aufstellen mußten. Bezogen auf die tatsächlich ausgeübte Kontrolle war diese Einschätzung jedoch wiederum sehr exakt, so daß diese Befunde insgesamt die motivationalen Defizite der Depressiven verdeutlichen, die Annahme der kognitiven aber nicht stützen können (Abramson, Alloy \& Rosoff, 1981, S. 43).

Alloy und Clements (1992) beschäftigten sich mit der Fragestellung, inwieweit die Neigung einer Person, der 'illusion of control' anheim zu fallen, Vorhersagequalität hinsichtlich einer verminderten Depressionsvulnerabilität beinhaltet. Wie von einigen Wissenschaftlern mittlerweile hypostasiert, verhilft diese Neigung in ihren Konsequenzen zu adaptiven und förderlichen Prozessen, die der geistigen und körperlichen Gesundheit zugute kommen; ,$\ldots$ these investigators have proposed that such biases and illusions enable individuals to maintain positive affect, self-regard, hope, and behavioral persistence in the face of life's obstacles and stresses and ... reduce susceptibility to depresssion and other illnesses“ (Alloy \& Clements, 1992, S. 241), obwohl gerade die Bereitschaft ,... in taking further actions to reduce the risk of various health problems ..." (Weinstein, 1982, S. 454) mit steigendem Optimismus abnimmt (ebd.). Die Frage der Kausalität, schon an anderer Stelle angesprochen (vgl. S. 97), nimmt auch hier eine bedeutende Stellung ein. Ihr liegt die Überlegung zugrunde, daß diese Illusion die Auswirkungen negativer Lebensereignisse abzumildern vermag und somit eine vor Depression schützende Qualität gewinnt (a. a. O., S. 235).

\footnotetext{
${ }^{22}$ Der Versuch wurde durch ein gelbes Licht eingeleitet; innerhalb der folgenden 5 Sekunden mußten sich die Versuchspersonen für oder gegen den Knopfdruck entscheiden, worauf als experimentelle Reaktion entweder ein grünes Licht aufleuchtete oder nichts geschah.
} 
So wurde in dieser Arbeit (Alloy \& Clements, 1992) der Grad der Vulnerabilität, bedingt durch realistische Kontrollwahrnehmung, beleuchtet. Nachdem die Versuchspersonen das dritte Experiment von Alloy und Abramson (1979; siehe auch S. 94) absolviert hatten, wurden sie mit einer unlösbaren Aufgabe konfrontiert, die aber in den Augen der meisten Versuchspersonen durchaus lösbar schien. Mit diesem Vorgehen wurde experimentell Versagen induziert. Um nicht auf die künstliche Laborsituation reduziert zu bleiben, wurden auch negative Lebensereignisse miterhoben, die entweder vor der ersten Untersuchung oder zwischen der ersten und zweiten Untersuchung, zwischen denen ein Zeitraum von einem Monat lag, erlebt wurden; parallel wurden zu den verschiedenen Zeitpunkten Stimmungsund Depressionsinventare ausgefüllt.

Tatsächlich erwies sich die 'illusion of control' als ein guter Prädiktor; je höher die Kontrolleinschätzung war, desto geringer war zum einen die negative Stimmungsreaktion nach der ungelösten Aufgabe ausgeprägt, zum anderen die Entmutigung nach negativen Alltagserlebnissen oder die Depressivität (vorausgesetzt, es lagen in dem Monatsintervall genügend negative Ereignisse vor, um diesem Risiko überhaupt ausgesetzt gewesen zu sein; Alloy \& Clements, 1992, S. 238-240). Doch trotz statistischer Signifikanz sind diese Anteile an der Varianz sehr gering, weshalb die Autoren die Möglichkeit in Betracht zogen, „... that the magnitudes of these variances are the best that can be expected; that is, the illusion of control may only provide a small degree of protection against depressive affect and symptoms after life stresses“ (Alloy \& Clements, 1992, S. 243).

\subsection{Erwartung und Vorhersage von Ereignissen}

'Illusion of control' wird als illusionäre Kontrollüberzeugung ausgeführt. Sie nimmt auch bei der Vorhersage von Eregnissen eine wichtige Stellung ein.

Langer (1975) prägte mit ihrer Veröffentlichung 'The illusion of control' (1975) den in der vorliegenden Arbeit bereits eingeführten Ausdruck, der nachfolgend in der Forschung für das Phänomen stand, sich im Besitz von mehr Kontrolle zu wähnen, als es der jeweiligen Realität entspricht (vgl. Fußnote 20, S. 95). Obwohl grundsätzlich der Unterschied zwischen Könnens- und Zufallsaufgaben jedermann geläufig ist, existieren bezüglich der Wahrnehmung konkreter Ereignisse fließende Grenzen; hier werden die klaren Grenzen des Konzepts - das Ergebnis der Könnensaufgaben ist durch das eigene Vermögen bestimmt, während es bei Zufallsaufgaben nicht kontrollierbar ist - aufgeweicht. Da diese Schwächen der eindeutigen Abgrenzung nicht zufällig zustandezukommen scheinen, sind die näheren Umstände von Interesse, die zu einer systematischen Verzerrung führen. 
Neben bestimmten Merkmalen des Aufgabentyps (s. u.) stellt der Glaube an eine gerechte Welt, in der Literatur als 'just world hypothesis' (Langer, 1975, S. 312) bezeichnet, einen Aspekt unter den denkbaren Einflußfaktoren dar, der in besonderer Weise Beachtung verdient. Menschen mit dieser Überzeugung gehen davon aus, daß Handlungen und deren Konsequenzen, ob kurz- oder langfristig, in der Wertigkeit auf gleicher Stufe stehen, daß also jeder „bekommt, was er verdient“. In dieser Welt, in der dem, der Gutes tut, Gutes widerfährt sowie dem schlechten Menschen Schlechtes, haben zufallsbestimmte Ereignisse nur sehr wenig Raum. Demnach können Ereignisse antizipiert werden und sind dadurch, in gewissem Sinne, kontrollierbar (Langer, 1975, S 312).

In einer Analyse der leistungsabhängigen Aufgabentypen scheint die Identifikation einiger Faktoren möglich, die zu der illusionären Kontrollüberzeugung führen. Insbesondere folgende Merkmale werden in den sechs Experimenten der Untersuchung von Langer (1975) berücksichtigt, ,... to introduce ... aspects of a skill situation ... [, wie zum Beispiel] choice, stimulus or response familiarity, passive or active involvement, competition - into a chance situation ..." (S. 313). Dies gelang, indem beispielsweise eine Vertrautheit mit verwendeten Lotteriescheinen hergestellt, durch die gespielte Unsicherheit des Gegenübers in einem Kartenspiel Kompetenzgefühle induziert oder durch Selbstziehen des Loses das Gefühl der aktiven Einbindung vermittelt wurde. Tatsächlich war in allen Experimenten das jeweilige Ergebnis jedoch vollständig zufallsabhängig. In den Resultaten (Experiment 1: Langer, 1975, S. 315; Exp. 2: a. a. O., S. 316 f.; Exp. 3: a. a. O., S. 318; Exp. 4: a. a. O., S. 319 f.; Exp. 5: a. a. O., S. 321; Exp. 6: a. a. O., S. 322) zeigte sich, daß nicht nur das Wissen um Kontingenzen handlungsrelevant ist, sondern auch die Wahrnehmung von Merkmalen, die sonst eher für eine Leistungsaufgabe typisch sind. Der Grund hierfür scheint in einer generellen Motivation des Menschen zu liegen, mit seiner Umwelt fertig werden zu wollen, so daß, in letzter Konsequenz, auch unkontrollierbare Umstände scheinbar kontrollierbar werden: „The more difficult a problem is, the more competent one feels in being able to solve it. The greatest satisfaction or feeling of competence would therefore result from being able to control the seemingly uncontrollable“ (Langer, 1975, S. 323).

Doch auch andere Gründe sind denkbar. Die Vermeidung des negativen Gefühls, das mit dem Erleben von Nichtkontrolle assoziiert ist, könnte ein Grund sein; ein anderer sicherlich, daß die Unterscheidung zwischen den beiden Aufgabentypen nicht immer problemlos geschieht. So sind durchaus in beiden oft Elemente des jeweils anderen enthalten. Beispielsweise kann man in einem Multiple-Choice-Verfahren durch zufallsgeleitetes Ankreuzen mitunter Resultate verbessern, in Las Vegas hingegen führt das Wissen darum, daß die Automaten am Flughafen öfter als die in der Stadt Gewinne ausschütten, zu größeren Ge- 
winnwahrscheinlichkeiten (Langer, 1975, S. 324). Eine weitere Konfundierung der gegensätzlichen Aufgabenarten ergibt sich aus dem Umstand, daß Ereignisse oft auf die Handlungen attribuiert werden, die ihnen unmittelbar vorausgingen; dies entspricht der Erfahrung im täglichen Leben, in dem sich Kausalitäten ${ }^{23}$ oft in dieser Konstellation zu erkennen geben.

Aufbauend auf der Arbeit von Langer (1975; siehe auch S. 105) untersuchten Golin, Terrel und Johnson (1977), inwieweit sich der von depressiven Personen empfundene Mangel an Kompetenz in deren Gewinnerwartung bezogen auf Zufallsaufgaben niederschlägt, wenn das Ausmaß der 'illusion of control' variiert. Hierzu wurde in einem Würfelspiel der Würfel entweder von der Person selbst oder dem Croupier geworfen, im Sinne Langers (1975) eine Variation des Merkmals 'aktive Einbindung'. Die geäußerten Erfolgserwartungen schwankten in deutlicher Abhängigkeit von der Zugehörigkeit zu einer der vier Gruppen, welche sich aus der Kombination des Depressionsgrades (depressiv/nichtdepressiv) und der Ausprägungen der 'illusion of control' (hoch/niedrig) ergaben. Depressive, die den Würfel selbst warfen, gaben geringe Gewinnerwartungen an, genauso wie die Nichtdepressiven, wenn der Würfel nicht von ihnen geworfen wurde; für die anderen beiden Kombinationsmöglichkeiten war die Gewinnerwartung vergleichsweise hoch. Es ergab sich neben einer Replikation der Befunde Langers (s. o.), daß die geringere Erwartung ,... for success is in accord with the view that depressed persons are characterized by a sense of personal incompetence ... “ (Golin, Terrell \& Johnson, 1977, S.441). Gleichzeitig zeigten sie nur eine geringe Ausprägung der illusionären Kontrollüberzeugung (vgl. auch Golin, Terell, Weitz \& Drost, 1979).

Eine „distorted perception of response-reinforcement ...“ (Klein \& Seligman, 1976, S. 12) gehört auf der einen Seite unmittelbar zu dem Phänomenbereich der Gelernten Hilflosigkeit, auf der anderen Seite ist sie aber auch mit dem Begriff der Depression unabdingbar verbunden. In einer Reihe von Untersuchungen zeigten Depressive und gelernt Hilflose, verglichen mit Nichtdepressiven bzw. nicht 'gelernt Hilflosen' (siehe Fußnote 19, S. 95), geringere Tendenzen, kontrollierbarem Lärm, dem sie in einer Shuttlebox ausgesetzt waren, zu entfliehen (Klein \& Seligman, 1976, S. 15 f.). Dies verdeutlicht die Parallelität der Erscheinungsformen, genauso wie der Befund, daß die Tendenz, aversiven Stimuli nicht aus-

\footnotetext{
23 'Kausalität' wird hier im Sinne des Allgemeinverständnisses gebraucht, nicht in einem wissenschaftstheoretischen Verständnis.
} 
zuweichen, bei beiden Gruppen abgemildert bzw. umgekehrt werden konnte, indem die Versuchspersonen durch die Konfrontation mit lösbaren Aufgaben 'therapiert' wurden.

Dieses Vorgehen wurde auf die Wahrnehmung von Kontingenzen übertragen, mit der Fragestellung, ,... whether distorted perceptions of response-reinforcement independence associated with depression and inescapable noise could be reversed by therapy with solvable problems“ (Klein \& Seligman, 1976, S.18). Die beteiligten Personen wurden der gleichen Prozedur wie im vorhergehenden Experiment ausgesetzt, mit der Abwandlung, daß nun nach der 'Therapie' Könnens- bzw. Zufallsaufgaben (zur weiteren Beschreibung der Aufgaben vgl. Gaber \& Hollon, 1980; siehe auch S. 109) zu bewältigen waren. Die Könnensaufgaben schienen jedoch nur durch das persönliche Geschick bestimmt, in Wirklichkeit wurde ihr Ergebnis dergestalt manipuliert, daß alle Versuchspersonen das gleiche $\mathrm{Ma} ß$ an Verstärkung erfuhren. Erhoben wurde das Ausmaß der Veränderung in der Erfolgserwartung über die einzelnen Versuche hinweg, welche bei Depressiven und 'gelernt Hilflosen' in der Folge tatsächlich weniger groß war, als bei ihren jeweiligen Vergleichsgruppen (a. a. O., S. 19 ff.). Dies bedeutet eine eindeutigere Realitätsorientierung der Depressiven im Vergleich zu den Nichtdepressiven, was auch für den Absolutwert ihrer Erwartung am Ende des Versuchs gilt (Klein \& Seligman, 1976, S.22).

Weitere Faktoren, die die Einschätzung der Erfolgserwartung beeinflussen, sind, neben dem Muster eines eventuellen Erfolgs (ob auf- oder absteigend; Dykman, Abramson \& Albright, 1991), die Lokalisation der Handlungsausführung und das 'setting', in dem die Handlungen stattfinden.

In welcher Weise sich die Veränderungen entlang der Dimension der Endpole 'öffentlich' bzw. 'privat' verhalten, war Gegenstand der Studie von Sacco und Hokanson im Jahr 1982. Sie zeigten, daß Depressive in einem privaten Umfeld im Vergleich zu Nichtdepressiven stärkere Änderungen vornahmen, im Gegensatz zu der öffentlichen Situation, in der diese weniger ausgeprägt waren (Sacco \& Hokanson, 1982, S. 380 ff.). Garber und Hollon (1980) beschäftigten sich bezüglich der Lokalisation mit dem Wahrnehmungsdefizit der Depressiven (siehe S. 108). Der Schwerpunkt lag hierbei auf der Frage, ob die Verzerrung bei Könnensaufgaben spezifisch für das eigene Ausführen zutrifft oder ob sie einem allgemeinen Glauben an Unkontrollierbarkeit entspringt. Letzteres entspräche in größerem Maße der Gelernten Hilflosigkeit, die von einem generalisierten Glauben an Nichtkontingenz ausgeht. In die gleiche Richtung zielt auch die häufig vorgenommene Interpretation von Studien, die wenig Änderungen in der Erfolgserwartung bei depressiven Personen fanden (vgl. z. B. S. 108): „In all these studies, the small expectancy changes in skill of depressed 
and helpless subjects have been interpreted as reflecting their general tendency to perceive responding and outcomes as independent" (Garber \& Hollon, 1980, S. 58).

Die Überlegung, inwieweit die Lokalisation eine Rolle spielt, mündet letztlich in die Frage, ob ,... depressed individuals believe they are universally or personal helpless“ (Garber \& Hollon, 1980, S. 58). Persönliche Hilflosigkeit würde das Gefühl der Depressiven widerspiegeln, eine Aufgabe zwar als grundsätzlich lösbar anzusehen, ohne aber selbst die entsprechenden Fähigkeiten dazu zu besitzen, während die universelle Hilflosigkeit ein Ausdruck für die Wahrnehmung der Aufgabe als zufallsabhängig wäre.

Hierzu implementierten Garber und Hollon (1980) ein experimentelles Design, das die von Klein und Seligman bereits verwendeten Könnens- und Zufallsaufgaben übernahm (1976; vgl. auch S. 107). Die Versuchspersonen mußten in der Zufallsbedingung raten, welches durch den Versuchsleiter willkürlich selektierte Diapositiv als nächstes erscheint, während es in der Könnensaufgabe scheinbar um die Geschicklichkeit im Hochziehen einer Plattform ging, ohne daß ein darauf positionierter Ball herunterfallen durfte; Erfolg und Versagen waren auch hier experimentell kontrolliert. Vor jedem Versuch wurde die Sicherheit des Patienten erhoben, Erfolg zu erleben oder, in der Bedingung, in der ein 'Vertrauter' die Aufgabe erfüllte, mit welcher Wahrscheinlichkeit diese Person erfolgreich sein würde. Die Ergebnisse wiesen eine Tendenz auf, welche eher für eine Selbstsicht der Depressiven als 'personally helpless' sprach (Garber \& Hollon, 1980, S. 60 ff.). Die Veränderung der Erfolgserwartung über die einzelnen Versuche hinweg war in der leistungsdeterminierten Versuchsbedingung recht groß, wenn es sich um die Einschätzung für eine andere Person handelte; Unterschiede zwischen Depressiven und Nichtdepressiven traten dabei nicht auf. Bezogen auf die Veränderung der Erfolgserwartung bei eigener Ausführung divergierten die Gruppen; die Depressiven zeigten vergleichsweise schwächer ausgeprägte Änderungen.

Nachdem bislang die Erwartung von Ereignissen im Vordergrund stand, richtet sich nun der Blick auf die Vorhersage der Wahrscheinlichkeit von Ereignissen. Diese bezieht sich sowohl auf die eigene wie auch auf eine andere Person, das Ereignis kann hierbei positiver oder negativer Natur sein.

Ungeachtet der Tatsache, daß verschiedene Forscher immer wieder auf die Verbindung von Depression und negativer Zukunftserwartung hinwiesen (vgl. Alloy \& Ahrens, 1987, S. 366), war der Nachweis der Evidenz bei konkreten Vorhersagen noch nicht erbracht. Aus dem Vergleich mit anderen Studien ergibt sich die Vermutung, daß die Zukunftssicht der Depressiven möglicherweise keine generalisierte negative Erwartung widerspiegelt, sondern nur auf die eigene Person bezogen ist. Dies stände auch mit Gedanken Beck's in Ein- 
klang, der von einem ,... systematic bias against the self ...“(vgl. Alloy \& Abramson, 1988, S. 225) ausgehend die Möglichkeit einräumt, daß Depressive für sich andersartige Erwartungen haben als für andere Personen. Ein ähnliches Phänomen ist mit der Begrifflichkeit der 'personally versus universal helplessness' umschrieben (s. o.).

Inwieweit bei gleichem Informationsniveau depressive Personen aber in einer konkreten Situation andere, das heißt negativere Zukunftserwartungen äußern, als Personen ohne depressive Symptomatik, versuchten Alloy und Ahrens (1987) herauszufinden, indem sie Depressive und Nichtdepressive auf der Grundlage der gleichen Hinweise Wahrscheinlichkeiten für ein ungewisses, zukünftiges Ereignis (Erfolg im Studium) schätzen ließen. Für die Betrachtung der Erwartungsspezifität wurde deutlich, daß ,... depressives' forecast for others as well as for themselves were more pessimistic than those of nondepressives .... [und daß] depressed subjects were also less likely than nondepressed subjects to predict that their own future outcomes would be more positive than those of similar others ..." (Alloy \& Ahrens, 1987, S. 374). So äußerte sich der Pessimismus der Depressiven hier tatsächlich globaler und generalisierter, als es die oben beschriebenen Überlegungen nahelegten. Die Bezeichnung Pessimismus bezieht sich jedoch nur auf den direkten Vergleich. Auf die Realität bezogen bewiesen die Depressiven auch hier in ihrer Selbstsicht eine Wahrnehmung, die frei von einem 'bias' war. Übereinstimmend mit diesen Befunden fanden Crocker, Alloy und Kayne (1988) innerhalb dessen, daß „,... depressed and nondepressed subjects differ in their perception of consensus ... [daß,] whereas nondepressed individuals perceive that positive events are more likely and negative events are less likely to happen to them than to others, depressed individuals do not show these self-enhancing comparisons between self and others" (S. 844).

$\mathrm{Zu}$ diesem Ergebnis kamen sie, indem sie Depressive und Nichtdepressive befragten, inwieweit positive, negative oder neutrale Ereignisse zum einen ihnen, zum anderen fremden Personen geschehen werden. Mit einem ähnlichen Design, jedoch ohne Bezug zur Depressivität, gelang es auch durch eine Studie von Weinstein (1980), Aussagen zu der optimistisch verzerrten Zukunftssicht der Nichtdepressiven zu machen. Die Auftretenswahrscheinlichkeiten von bestimmten Ereignissen für sich und für Mitstudenten beurteilend wurde deutlich, daß die Beziehung zwischen gefundenen Werten und positiven Ereignissen ,$\ldots$ indicates that subjects tend to believe that their own chances are greater than average [und negative betreffend] ... their chances are less than average .... [und damit] the existence of strong optimistic tendencies is clear in both“ (Weinstein, 1980, S. 811). Desgleichen war die Überschätzung in einer Längsschnittstudie immens, die Valone, Griffin, Lin und Ross (1990) durchgeführt hatten; im Verhältnis der Vorhersagen zu der Realität „,... the 
objective odds were about 3:1 against the subject predictions“ (S. 591), und auch Dunning, Griffin, Milojkovic und Ross (1990) berichteten im Anschluß an fünf Untersuchungen im sozialen Kontext: ,... people proved to be markedly overconfident in general“ (S. 578).

Befunde, nach denen ,... negativity in thoughts that accompanies depression is restricted to thoughts about oneself and does not extend to thoughts about others" (Pietromonaco \& Markus, 1985, S. 804), widersprechen in Teilbereichen den zuvor angeführten. Angesichts der Wichtigkeit kognitiver Aspekte für die Entstehung und Aufrechterhaltung der depressiven Symptomatik versuchten Pietromonaco und Markus (1985), den Ursprung und den Inhalt von Kognitionen Depressiver näher zu beleuchten. Hierzu wurden neben der Frage, inwieweit negative Gedanken spezifisch auf die eigene Person bezogen oder generalisiert sind, zwei weitere Aspekte untersucht: zum einen, ob seitens der Depressiven die Sicht sozialer Ereignisse negativer und eventuell weniger differenziert ist, zum anderen, welche Aktivitäten oder Handlungen mit der größten Wahrscheinlichkeit negative Gedanken in Depressiven hervorzurufen vermögen (Pietromonaco \& Markus, 1985, S. 800).

Im experimentellen Vorgehen wurden mittels Diapositiven Ereignisse beschreibende Sätze dargeboten, die inhaltlich entlang zweier Dimensionen angeordnet waren, beschreibbar durch die Pole 'glücklich' und 'traurig' bzw. 'sozial' und 'nicht sozial'. In verschiedenen Bedingungen galt es, sich das Ereignis vorzustellen, zu erinnern oder in bezug auf mögliche Interpretationen zu beurteilen, und dies sowohl hinsichtlich der eigenen Person wie auch in bezug auf eine fremde Person, welche den Status eines Bekannten ${ }^{24}$ haben sollte. Die Ergebnisse wiesen die eingangs erwähnte Tendenz auf: „The depressed subject ... was better able than the nondepressed subjects to picture someone saying that she was selfish ... believed that someone was likely to say that she was selfish ... that this statement would be made because she was indeed selfish“ (Pietromonaco \& Markus, 1985, S. 804). Ob die Sätze eine soziale Situation betrafen oder sozial neutral waren, hatte keine Auswirkung auf die Ergebnisse. Die Differenzierung in 'glücklich' und 'traurig' bewirkte in der Bedingung, daß Depressive auf sich selbst bezogen negative oder traurige Ereignisse stärker als für andere gewichteten. Sie wurden beispielsweise besser vorgestellt oder erinnert (Pietromonaco \& Markus, 1985, S. 802 f.).

Durch den Umstand, daß in der Sicht für andere weder die Vorstellung noch die Erinnerung den Einflüssen eines 'bias' unterliegt, scheint es unwahrscheinlich, daß die negativere

\footnotetext{
${ }^{24}$ Es sollte keine Person sein, die dem Untersuchten zu nahe stand, da mit zunehmender Nähe die Unterschiede zwischen der Verarbeitung von Informationen, die das Selbst, und denen, die die andere Person betreffen, abnehmen (Pietromonaco \& Markus, 1985, S. 801).
} 
Sicht Depressiver durch eine höhere Verfügbarkeit oder Abrufbarkeit von negativen Gedankeninhalten an sich erklärbar ist. Die Möglichkeit einer in Abängigkeit vom Bezugspunkt der Gedanken differierenden Verfügbarkeit dieser Gedankeninhalte wird durch Befunde gestützt, nach denen selbstbezogene Kognitionen anders behandelt wurden als diejenigen, die andere betrafen.

\subsection{Attribution}

Kausalattributionen für hypothetische oder reale Ereignisse werden untersucht. Die Herangehensweise ist hierbei phänmenologisch. Die selbstwertsteigernde Funktion von Attributionsmustern als eine zentrale Annahme im Komplex des 'self-serving bias' wird diskutiert.

Kausalattributionen für hypothetische oder reale Ereignisse sind Gegenstand der folgenden Untersuchungen. Mit ihnen wird nun ein Bereich eröffnet, der im Gegensatz zu dem ersten ('Einschätzung der Kontingenz', Kap. 2.3.2.3, S. 93) fast ausschließlich auf relative Aussagen beschränkt ist. Die Realität, die scheinbar wahre Kausalität, bleibt oft hinter den augenscheinlichen Dingen verborgen. Des weiteren ist die gesamte Herangehensweise 'phänomenologisch' geprägt, da letztlich für die Folgen der Attributionen der einzelnen Person lediglich das in der Wahrnehmung repräsentierte Abbild der Realität von Bedeutung ist. Das Gefühl der Kompetenz, das aus internal attribuiertem Erfolg in einer schwierigen Aufgabe resultiert, zeigt sich unbeeinflußt von einer alternativ vorstellbaren Kausalität, auch wenn diese in der objektiven Welt außerhalb der Person bestimmend war (vgl. Weiner, 1988, S. 311 ff.).

Obwohl die Gelernte Hilflosigkeit in der ätiologischen Grundlagenforschung unter denen, die nicht dem klinischen Bereich entstammen, eines der bedeutendsten Erklärungsmodelle darstellt, (siehe auch Kap. 2.3.1.3.2, S. 74), sind doch manche Punkte auch durch sie nicht befriedigend beantwortet. Attributionstheoretisch denkend überrascht vor allem die Befundlage bezüglich bestimmter Attributionsmuster, die depressiven Personen bei Mißerfolg internale Attributionen nachweist; ,if people perceived an outcome to be uncontrollable, then logically they should not blame themselves for failure at such a task" (Sweeny, Anderson \& Baily, 1986, S. 974).

Einen Ausweg aus dieser Problematik bietet die Attributionstheorie, deren Annahmen zum Teil in die Theorie der Gelernten Hilflosigkeit eingearbeitet wurden (Kuiper, 1978; vgl. auch S. 75). Sie operiert, wie in Kap. 2.3.1.3.2 dargestellt (S. 74), mit drei Dimensionen (vgl. Weiner, 1988, S. 217 ff.), entlang derer die für die Depressionsforschung wichtigen Attributionen vorgenommen werden. Dabei ist jede Dimension mit einem spezifischen Ausschnitt aus dem Spektrum der depressiven Symptomatik assoziiert. 
Die vorgenommenen Attributionen spiegeln jedoch, ganz im Gegensatz zu der ursprünglichen Intention des Attribuierenden, nur selten die ungefärbte Realität wider. Ein Phänomen in diesem Zusammenhang, in der Literatur oft unter der Bezeichnung 'selfserving bias' (Bradley, 1978) geführt, ist die, ,... tendency for individuals to make selfattributions for their own positive behaviours and external attributions for their own negative behaviours“ (Bradley, 1978, S. 56), oder, wie Miller und Ross im Jahre 1975 konstatierten: ,... to explain ... behavior in terms that 'flatter us' and 'put us in a good light'“ (S. 213). Doch scheinen auch diese Phänomene nicht auf alle Menschen gleichermaßen zuzutreffen; so kann eine Differenzierung nach dem Grad der Depressivität diese Erscheinung klarer in ihren Wirkbereichen abgrenzen.

Kuiper (1978) erhob Kausalattributionen bezüglich Erfolg und Versagen von weiblichen Studenten, die zuvor eine Wort-Assoziations-Aufgabe bearbeitet hatten und dabei ein unterschiedliches Maß an Verstärkung erfuhren. Der bedeutendste Befund war, daß entgegen der Vorhersage der Gelernten Hilflosigkeit und konsistent mit schon referierten Ansätzen (siehe S. 112) Depressive bei Mißerfolg internal attribuierten. Damit wurde ein Tatbestand beleuchtet, der bis zu diesem Zeitpunkt noch ungeklärt war. In der vorangegangenen Forschung (vgl. Kuiper, 1978, S. 243) hatte sich lediglich gezeigt, daß Depressive schlechtere Leistungen zeigten, wenn sie gemäß der Versuchsanordnung internal attribuierten, ohne jedoch Aussagen darüber machen zu können, welche Attributionsmuster Depressive gebrauchen, wenn ihnen die experimentelle Variation diesbezüglich Freiheit gab. Die Nichtdepressiven hingegen zeigten die Tendenz, für positive Ergebnisse stärker als bei Versagen die eigene Person verantwortlich zu machen (Kuiper, 1978, S. 241 f.). In dieser Asymmetrie drückt sich empirisch die als 'self-serving bias' bezeichnete und den Selbstwert steigernde Verzerrung aus.

Die Entsprechung von Erfolgs- und Versagenserfahrungen in der Selbstbewertung ('selfevaluation') war der Gegenstand des Interesses von Sackheim und Wegner, als sie 1986 in zwei Untersuchungen depressive, euthymische und schizophrene Personen untersuchten. Mit dem Anspruch, ein weites Spektrum zu erfassen, arbeiteten sie sowohl mit hypothetischen Ereignissen, die in Form von Geschichten Alltagserfahrungen betrafen, als auch mit in einer Laborsituation erzeugten realen Ereignissen. Da hypothetische Ereignisse den Nachteil in sich bergen, daß Personen bei diesen eventuell anders attribuieren als bei tatsächlichen, während die Laborsituation künstlich und die Konsequenzen zumeist weitgehend unerheblich sind, war die Hoffnung, daß über beide Situationen hinweg ähnliche Ergebnisse höhere Aussagekraft gewinnen, daß also „,... with the presumption that consistent 
group differences across tasks would provide firmer grounds for inferring reliable differences in attributional processes“ (Sackheim \& Wegner, 1986, S. 554).

Geschichten mit vorzustellenden Ereignissen waren mit der Intention geschrieben, daß „... positive and negative outcomes be equivalent in regard to the dimensions of controllability, praise/blame, and outcome intensity“ (ebd.). Die Versuchspersonen hatten sich hierbei in die Lage der Hauptperson zu versetzen. Die tatsächlichen Ereignisse bestanden aus der Fortführung von Zahlenreihen, deren Ergebnisse in Abhängigkeit vom Verstärkungsplan als 'richtig' oder 'falsch' rückgemeldet wurden. In der Auswertung wurde deutlich, daß auch hier eine Verzerrung zu beobachten war, die zum einen den Annahmen des 'selfserving bias' (vgl. S.113), zum anderen denen der 'depressive realism hypothesis' entspricht: Die Nichtdepressiven beurteilten die ausgeübte Kontrolle als größer, die positiven Implikationen für den Selbstwert als zahlreicher ${ }^{25}$ und das Ereignis als intensiver, wenn die Ergebnisse positiver Natur waren, womit sie sich in ihren ,... attributions for positive ... [and] negative outcomes ... far from evenhanded“ (a. a. O., S. 559) zeigten. Die Depressiven, frei von dieser Tendenz, divergierten in ihrer Beurteilung nicht in Abhängigkeit von Erfolg und Mißerfolg (Sackheim \& Wegner, 1986, S. 556).

Mit dem Design einer Langzeitstudie versuchten Hamilton und Abramson (1983), Veränderungen über eine bestimmte Zeit hinweg in kognitiven Mustern bei Depressiven zu beobachten. Nach der reformulierten Theorie der Gelernten Hilflosigkeit wurden für die Depressiven bestimmte Attributionsmuster erwartet ('internal', 'stabil', 'generalisiert'/'global'), wobei sowohl in dieser als auch in der Beck'schen Theorie (vgl. Beck, Rush, Shaw \& Emery, 1994) ,.... the concept of a traitlike depressive cognitive style [ein konzeptuell wichtiges Element darstellt,] that characterizes some depression-prone individuals and persists beyond remission of the person's current depressive episode unless specifically identified and modified“ (Hamilton \& Abramson, 1983, S. 173). Nach Beck handelt es sich bei diesen kognitiven Stilen um die von ihm hypostasierten ‘dysfunktionalen Überzeugungen' (Beck, Rush, Shaw \& Emery, 1994; siehe auch Kap. 2.3.1.3.2, S. 73).

Mit Hilfe eines Fragebogens wurden Attributionsmuster von Personen einer New Yorker Klinik erhoben, die weder organische Befunde aufwiesen, noch an einer bipolaren Störung litten oder mit Elektrokrampftherapie behandelt wurden. Als Vergleichsgruppe dienten

\footnotetext{
25 „They indicated that they merited more praise for positive outcomes than blame for negative outcomes“
} (Sackheim \& Wegner, 1986, S. 558). 
nichtdepressive Personen, die sich nach Aufruf freiwillig meldeten. Untersucht wurden die Versuchspersonen unmittelbar nach der Aufnahme, während die zweite Untersuchung kurz vor der Entlassung stattfand; für die Kontrollgruppe wurde ein vergleichbarer Zeitraum gewählt.

Die Ergebnisse des Vergleichs über die Gruppen und die Zeitpunkte hinweg legen nahe, daß ,... cognitive pattern exhibited by depressives during their depressive episode at Time 1 were not a general feature of psychopathology, at least in terms of extremity" (Hamilton \& Abramson, 1983, S. 181). Vor allem in bezug auf die 'depressive realism hypothesis' ist das Resultat von Bedeutung, daß diejenigen Werte ${ }^{26}$, deren Höhe die Ausprägung eines 'selfserving bias' widerspiegeln, bei den Depressiven erst gering waren, sich dann aber, nach erfolgter Behandlung und Besserung der Symptome, den über beide Erhebungszeitpunkte hinweg gleichbleibend hohen Werten der nichtdepressiven Vergleichsgruppe anglichen (Hamilton \& Abramson, 1983, S. 177).

Mit klinisch Depressiven arbeitete ein Jahr zuvor auch ein anderer Forscherkreis (Raps, Reinhard, Peterson, Abramson \& Seligman, 1982), der sich neben der Ermittlung der Attributionsmuster zur Aufgabe machte, ,.... to investigate causal 'evenhandness' among severly depressed individuals“ (Raps, Reinhard, Peterson, Abramson \& Seligmann, 1982, S. 103). Die Erwartung wurde bestätigt, die Resultate bewiesen eine größere Ausgeglichenheit seitens der Depressiven bezüglich der Attributionen nach guten und schlechten Ereignissen (a. a. O., S. 104). Hinsichtlich der Attributionsmuster wurde auch hier in stärkerem Maße verglichen mit den Nichtdepressiven - eine stabile, internale und generalisierte Sicht bei den Depressiven gefunden.

Da die selbstwertsteigernde Funktion von Attributionsmustern als eine zentrale Annahme im Komplex des 'self-serving bias' gilt, erhoben Tennen und Herzberger 1987 den Selbstwert zum zentralen Forschungsgegenstand einer Untersuchung. Die durch ein hohes Selbstwertgefühl ('self-esteem') gespeiste Erwartung, Erfolg zu haben, sieht sich bei Versagen mit einer anderen als der antizipierten Realität konfrontiert; darauf reagierend wird die Diskrepanz, konsistent mit dem phänomenologischen Ansatz (vgl. Weiner, 1988 ; siehe auch Kap. 2.3.2.3.3, S. 112), in der Wahrnehmung geglättet, indem die negativen Ereignisse beispielsweise auf Zufallseinflüsse zurückgeführt werden. Nichtdepressive zeigen demgemäß keinen 'bias', da sie keine anfängliche Erfolgserwartung haben. Aus diesem Blick-

\footnotetext{
26 ‘ASQ composite difference score’ (vgl. Hamilton \& Abramson, 1983, S. 176)
} 
winkel erscheint der depressionsätiologische Teilaspekt in einem neuen Licht: Depressive „... suffer from a breakdown in their motivation to maintain self-esteem“ (Tennen \& Herzberger, 1987, S. 72).

Nachdem die beteiligten Versuchspersonen die Fragebögen ausgefüllt hatten, durch die der Grad der Depression (BDI; vgl. auch Kap. 3.3.2.7, S. 145), die Attributionsmuster (ASQ), das Ausmaß der Angst (STAI) und die Selbsteinschätzung (SDI) ermittelt wurden, zeigte sich in der Auswertung, daß sowohl die Attributionsmuster als auch die Ausgeglichenheit in der Attribution nach positiven und negativen Ereignissen in überzeugender Weise nur durch die Selbstachtung vorhergesagt werden konnten (Tennen \& Herzberger, 1987, S. 77). So erweist sich hier, daß eventuell nicht die Depression als solche, sondern die durch die Depression hervorgerufene erniedrigte Selbstachtung für die besagten Prozesse verantwortlich ist.

Im Rahmen der Erforschung eventueller Verzerrungen in der Wahrnehmung von Konsens erwartete Campbell (1986), daß diese in Abhängigkeit von der Attribution, der Relevanz derselben und dem Selbstwertgefühl bzw. dem Grad der Depressivität variieren. Als Kern der Untersuchung hatten die teilnehmenden Personen auf einer fünfstufigen Skala das Ausmaß der persönlichen Übereinstimmung zu verschiedenen Aussagen und Fähigkeiten anzugeben, die ihnen auf einer Liste aufgeführt vorlagen. Anschließend galt es, die Prozentzahl der Collegestudenten zu schätzen, die die gleiche Meinungen bzw. gleiche Fähigkeiten besitzen. Das Resultat war, daß ,low self-esteem and DEP were less likely to overestimate consensus for their opinions and to underestimate consensus for their abilities" (Campbell, 1986, S. 290). Die größere Ausgeglichenheit war wieder auf der Seite der Depressiven zu beobachten.

\subsection{Wahrnehmung und Erinnerung von Rückmeldungen}

Wahrnehmung und Erinnerung von Rückmeldungen stehen im Fokus des folgenden Forschungsbereichs. Klassische Bpressionstheorien gehen von einer negativen Verzerrung aus, was in zahlreichen Studien nicht bestätigt werden konnte. Negative Schemata Depressiver werden nicht durch eine übersensitive Verarbeitung negativer Stimuli der Umwelt ausglöst, vielmehr scheinen die positiven Schemata schwerer aktiviebar.

Ein weiteres Feld, auf dem Unterschiede zwischen Depressiven und Nichtdepressiven im Hinblick auf ihren Realitätssinn gefunden wurden, stellen Wahrnehmungen und Erinnerungen von Rückmeldungen ('feedback') dar. Die Rückmeldung bezieht sich hierbei auf verschiedene Aspekte und Zeitpunkte. Neben der unmittelbaren Rückmeldung, die sich sowohl auf Personenimmanentes als auch auf die Ausführungsgüte einer Aufgabe beziehen kann, ist auch die Veränderung von Rückmeldungsinhalten über die Zeit sowohl für die Wahrnehmung als auch für die Erinnerung von Wichtigkeit. 
Gemäß der Annahmen, die der Kognitiven Theorie zugrundeliegen, ist bei den Depressiven insofern eine Verzerrung in der Informationsverarbeitung $\mathrm{zu}$ erwarten, als neutrale Stimuli mit negativen Einschlägen wahrgenommen bzw. erinnert werden, oder daß negative Rückmeldungen negativer aufgenommen werden, als sie es tatsächlich sind (vgl. Kap. 2.3.1.3.2, S. 73).

DeMonbreun und Craighead (1977) untersuchten ambulante Patienten ('outpatient'). Die handlungsleitende Vermutung war, daß die Wahrnehmung eines neutralen Reizes negative Verzerrungen aufweist. Hierfür war die Rückmeldung in dem Versuch (DeMonbreun und Craighead, 1977, S. 314 ff.) experimentell kontrolliert und positiv. Nachdem die Versuchspersonen unscharfe Bilder von unsinnigen Silben sahen, die nicht eindeutig erkannt werden konnten, entschieden sie sich nach der Darbietung eines weiteren Diapositivs mit vier möglichen Alternativen für diejenige Silbenkonstellation, die nach ihrer Einschätzung der auf dem vorangegangenen Bild in Schreibweise und Aussprache am ähnlichsten war. Die Rückmeldung erschien in Form eines grauen Bildes, das in angeblich vier verschiedenen Helligkeitsstufen auftauchte. Jede Helligkeitsstufe stand für eine spezifische Rückmeldung: zwei Grautöne für hundert- und fünfundsiebzigprozentige Akzeptanz der von der Versuchsperson gewählten 'Alternative mit der größten Ähnlichkeit', weitere zwei für die gleiche Abstufung in der Nichtakzeptanz.

Entscheidend war die Existenz eines fünften, den Versuchspersonen unbekannten Diapositivs in einem Grauton, dessen Helligkeitsstufe zwischen denen der beiden Zweiergruppen lag; es stellte somit ein neutrales Feedback dar und sollte über die oben erwähnten Verzerrungstendenzen Aufschluß geben. Die Ergebnisse fanden sowohl in der unmittelbaren Wahrnehmung positiver Rückmeldung als auch in der Wahrnehmung des neutralen Stimulus keine Unterschiede zwischen Depressiven und Nichtdepressiven. Dadurch, daß die Nichtdepressiven sich nicht überschätzten, findet zwar die Annahme der 'Overconfidence' keine Unterstützung, doch in noch stärkerem Maße wurde die negative Verzerrung durch die Depressiven widerlegt (DeMonbreun \& Craighead, 1977, S. 314 ff.). Absolut gesehen bewiesen die Depressiven ihre Realitätsnähe, indem sie das auf eine Aufgabe bezogene, positive Feedback realitätsgerecht einschätzten.

Klare Hinweise für die Evidenz einer positiven Verzerrung seitens der Nichtdepressiven erhielten hingegen Vestre und Caulfield (1986). In ihrer Analyse der erhobenen Daten zeichnete sich der deutliche Trend $a b,, \ldots$ that nondepressed subjects distort feedback in a positive direction and appear to illustrate the 'normal' positive biasing effects ... the depressed subjects were more realistic in their ratings of how their personality interpretation had 
depicted them“ (Vestre \& Caulfield, 1986, S. 35). Die untersuchten Personen hatten zuvor eine neutrale, nicht eindeutige ('ambiguous') Rückmeldung hinsichtlich der eigenen Person bekommen, die angeblich nach der Eingangsuntersuchung von ihnen angefertigt worden war; tatsächlich handelte es sich um 15 allgemeine Aussagen, die dem MMPI (vgl. Grubitzsch, 1991) entnommen waren.

Mit der Äußerung ,,it is noteworthy that 'characteristic processing' $\left[{ }^{27}\right]$ interpretations of depressive cognition have not been limited to Beck's schema theory but extend even to findings that run counter to his view“ (Dykman, Abramson, Alloy \& Hartlage, 1989, S. 431) implementierten Dykman, Abramson, Alloy und Hartlage (1989) ein differenzierteres Forschungsvorgehen. Nicht mehr der Annahme charakteristischer Verarbeitungsmuster Depressiver oder Nichtdepressiver verpflichtet, galt es, nuancierter die Umstände für die mögliche Existenz eines 'bias' seitens der Depressiven bzw. Nichtdepressiven zu isolieren. Die handlungsleitende Vermutung sah die Unterschiedlichkeit nicht mehr in der Informationsverarbeitung als solcher, sondern in der Zahl und dem Inhalt der Schemata, in die neue Informationen integriert ('assimilate') werden (Dykman, Abramson, Alloy \& Hartlage, 1989, S. 432).

Bezogen auf die hypostasierte Negativität seitens Depressiver wäre die Implikation dieser Annahme, daß nicht die Informationsverarbeitung negative Verzerrung bewirkt, sondern bei ihnen lediglich mehr negative Schemata vorhanden sind, wodurch die Wahrscheinlichkeit, daß neue Informationen in bzw. durch ein negatives Schema assimiliert werden, ansteigt. In diesem Rahmen widersprechen diejenigen Befunde von Studien, die die durch Beck postulierte Verzerrung nicht bestätigen (vgl. DeMonbreun \& Craighead, 1977; siehe auch S. 117), der Kognitiven Theorie nur in dem Maße, in dem „... one views Beck’s theory as a characteristic processing theory rather than as a schema theory“ (Dykman, Abramson, Alloy \& Hartlage, 1989, S. 432). Diese Gedanken stellen die Grundannahmen des depressiven Realismus in ein neues Licht; wären sie zutreffend, handelte es sich nicht mehr um einen größeren Realitätssinn bei Depressiven, basierend auf einer realitätsgerechteren Verarbeitung, sondern lediglich um das Vorhandensein zahlreicherer realitätsadaptierter Schemata.

Den empirischen Anteil ihres Ideengangs machte die Betrachtung des Kodierens und des Erinnerns zweierlei Arten von Rückmeldungen aus, die eindeutigen und die mehrdeuti-

\footnotetext{
${ }^{27}$ Der Begriff 'charakteristisch' wird hier im Sinne von 'jederzeit' oder 'über alle Situationen hinweg' gebraucht.
} 
gen ('ambiguous'). Hierbei interessierten die Fragen, wie es sich zum einen mit eventuellen, realitätsverzerrenden Tendenzen ${ }^{28}$ bei einer der beiden Gruppen verhält, zum anderen, ob Depressive in anderer Weise als Nichtdepressive verarbeiten oder, wie oben ausgeführt, lediglich Schemata mit anderen Inhalten besitzen. Die Betrachtung erfolgte gleichzeitig in drei verschiedenen Richtungen, jeweils unter Bezug auf die Verzerrung ('bias'): Die Kodierung mehrdeutiger Rückmeldung, die Verzögerung der Antwort bei eindeutiger Rückmeldung und die Erinnerung an diese. Im ersten Durchlauf wurden die Schemata mit dem Ziel erhoben, diejenigen Bereiche herauszufinden, in denen Depressive eine größere Anzahl negativer Schemata aufweisen.

Die Kodierungsverzerrung wurde über Feedbackpaare verschiedener Wertigkeiten mit der Hypothese erhoben, daß vom Schema abweichende Rückmeldungen in die Richtung des Schemas 'korrigiert', das heißt verzerrt, werden. Die Operationalisierung des 'bias' in der Antwortverzerrung gelang mit Hilfe der Annahme, daß ,... characteristic processing bias ... would result in a linearly increasing delayed response (or 'resistance') to feedback whose valence was increasingly inconsistent with that bias" (Dykman, Abramson, Alloy \& Hartlage, 1989, S. 433). Die Verzerrung in der Erinnerung über alle Rückmeldungen hinweg wurde durch das Verhältnis der Anzahl erinnerter positiver bzw. negativer Rückmeldungen zu der Anzahl der tatsächlichen Rückmeldungen ermittelt. Das Ergebnis, innerhalb dessen ein Teilaspekt beispielsweise in der Anmerkung ,,interestingly, our findings that depressed subjects in the schema-discrimination condition were significantly less biased overall in their encoding of ambiguous feedback than were nondepressed subjects in this same conditions is reminiscent of the depressive realism-nondepressiv optimism results that have been obtained in a number of previous studies“ (Dykman, Abramson, Alloy \& Hartlage, 1989, S. 441) Ausdruck fand, spricht deutlich für die Verarbeitungsart des 'schematic processing' und verwirft damit die Vorstellung einer 'charakteristischen' Verarbeitungsweise: die Verzerrrung war mit der Bedeutung ('valence') der jeweiligen Schemata viel stärker assoziiert als mit der Gruppenzugehörigkeit (depressiv/nichtdepressiv).

Kowalik und Gotlib (1987) fokussierten in ihrer Untersuchung die Interaktion von Ehepaaren. Da Depressive mehr negative und weniger positive Inhalte erinnerten, schien eine negative Verzerrung vorzuliegen - wenn nicht eine weitere Beobachtung dazugekommen wäre, daß sie im Vergleich zu Nichtdepressiven in der beobachteten Interaktion tatsächlich

\footnotetext{
${ }^{28}$ Negativer 'bias' seitens der Depressiven bzw. positive Verzerrung bei den Nichtdepressiven entsprechend der 'depressive realism hypothesis'
} 
weniger angenehme Kommunikationsmerkmale erlebten (vgl. auch 2.3.2.2.2, S. 92). So zeichnete sich insgesamt das Bild $a b$, daß ,... these findings undermine those cognitive theories which posit that depressed individuals distort their world negatively“ (Kowalik \& Gotlib, 1987, S. 133).

Buchwald (1977) bezog sich mit der Anmerkung, daß ,this interpretation rest on the assumption that subjects' reports of the occurence of pleasant events are accurate" (Buchwald, 1977, S. 443) auf die Annahme Seligmans, depressive Störung sei mit geringerer Anzahl positiver Erlebnisse assoziiert (vgl. Kap. 2.3.1.3.2, S. 73), und rückte die Spanne zwischen Annahme und Vermutung in das Zentrum seiner Studie. Indem die teilnehmenden Personen die Summe der positiven Rückmeldungen schätzen mußten, die ihnen in der Versuchsaufgabe zuteil geworden waren, zeigte sich einerseits eine Unterschätzung der Anzahl positiver Rückmeldung durch die Depressiven, andererseits eine Überschätzung derselben durch die Nichtdepressiven (a. a. O., S. 445).

Ausgeglichener war die von der Verarbeitungstiefe abhängige Erinnerung seitens Depressiver hinsichtlich selbstbezogener Aussagen in Verbindung mit Erfolg und Mißerfolg in einer Arbeit von Ingram, Smith und Brehm (1983), in der Nichtdepressive „,... recalled significantly more positive self-relevances than did those who were given failure feedback" (Ingram, Smith und Brehm, 1983, S. 417). Widersprochen wurde damit der Vermutung, negative Schemata Depressiver würden durch eine übersensitive Verarbeitung negativer Stimuli der Umwelt ausgelöst; vielmehr scheinen die positiven Schemata schwerer aktivierbar zu sein.

In dem gleichen Jahr fanden Nelson und Craighead (1977), die in ihrer Studie zum einen zusätzlich die Frequenz der Rückmeldung variierten, zum anderen anstatt nur mit positiver Rückmeldung mit Bestrafung und Belohnung ('reinforcement') arbeiteten und darüber hinaus Selbstbelohnung bzw. -bestrafung als Untersuchungsgegenstand einführten, unter anderem heraus, daß die Einschätzung der Nichtdepressiven in der Bestrafungssituation „... did not differ significantly from 12 (the actual rate), the nondepressed subjects significantly underestimated the amount of negative feedback they had received..." (Nelson \& Craighead, 1977, S. 384). Es wurde jedoch auch eine gewisse Verzerrung durch die Depressiven festgestellt, die sich bei hoher Frequenz der Rückmeldung mehr an Bestrafung als an Belohnung erinnern konnten, während sie sich jedoch nicht in einem höheren Maße bestraften, als es die Nichtdepressiven taten. 
Um eventuelle, versuchsbedingte Verzerrungen zu umgehen, die beispielsweise bei Nelson und Craighead (1977; siehe auch S. 120) eine Rolle gespielt haben könnten, indem die Erinnerung an die Rückmeldungen nicht mehr nur durch die Aufgabe, sondern auch durch grundsätzlichere Tendenzen bei Depressiven, Erinnerung an gute Leistungen über die Zeit herabzumindern, gesteuert war (vgl. Coyne \& Gotlib, 1983, S. 482 ff.), ließen Dennard und Hokanson (1986) die Kontingenz von Handlung und Verstärkung unmittelbar nach der Aufgabe einschätzen. Mit zweiwöchiger Verzögerung, wurde die Erinnerung an positive bzw. negative, adjektivische Items geprüft. Hinsichtlich der Einschätzung ergab sich, „... that with increasing levels of BDI score subject groups display progressively less of the self-enhancing, positively skewed judgments exhibited by nondysphoric subjects ... [und auch bezüglich der Erinnerung an die Items nach zwei Wochen] the results do not, to any appreciable degree, reflect negatively distorted judgments or memory functions among subjects scoring in the moderately depressed range BDI“ (Dennard \& Hokanson, 1986, S. 384). Lediglich in der Versuchsbedingung der niedrigen Verstärkungsrate (25\%) unterschätzten die Depressiven die tatsächliche Verstärkungsquote.

Loewenstein und Hokanson (1986) führten mit Personen verschiedener Depressionslevel eine standardisierte soziale Interaktion durch, in deren Anschluß situationsbezogene Kognitionen über 'sich', 'den anderen' und 'wie der andere einen selbst sieht' erhoben wurden. Personen mit leichten und mittleren Depressionen ('mildly'/'moderately') zeigten in der Untersuchung ein schlechteres Erinnerungsvermögen an persönliche Rückmeldungen im Vergleich zu Nichtdepressiven. Die Erinnerung war in beiden Gruppen unverzerrt (Loewenstein \& Hokanson, 1986, S. 452 ff.). Damit ist auch im Bereich der sozialen Interaktion, in dem sich die Rückmeldung nicht mehr auf die Güte der Ausführung einer Aufgabe, sondern auf unmittelbar mit der Person assoziierte Eigenschaften bezieht, die Existenz des depressogen wirkenden 'bias' in Frage gestellt.

\subsection{Selbstbewertung}

Das zentrale Merkmal dieses Bereichs ist die Selbstbewertung. Aussagen zur Realität können nur idiographisch getroffen werden. Selbstaufmerksamkeit erhöht die Genauigkeit der Selbsteinschätzung.

In dem Forschungsbereich, dessen konstituierendes Merkmal die Beschäftigung mit der Selbstbewertung von Personen ('self-evaluation') ist, können die erhobenen Einschätzungen an der wahren, tatsächlichen Realität - im Sinne der Realisten (vgl. Kap. 2.3.2.2.1, S. 90) - nicht objektiviert werden. Aussagen zu einer Realitätsorientierung, wie sie im Kontext dieser Ausführung gemacht werden, geschehen nur idiographisch, teilweise vergleichend, niemals aber absolut. 
Wie bereits im Rahmen der Kontingenzschätzung (Kap. 2.3.2.3.1, S. 93) angesprochen, konfrontierte Vázquez (1987) Probanden mit personenbezogenen Aussagen positiver und negativer Art, die entweder die eigene Person oder eine andere betrafen. Hinsichtlich der Selbstbewertung ist bemerkenswert, daß Nichtdepressive, akkurat in der Einschätzung des Kontingenzgrades negativer Sätze, ,... overestimated it in the positive conditions .... On the other hand, depressed subjects judged the degree of contingency accurately in the positive conditions but ... did not overestimate the actual degree of contingency when negative sentences were presented“" (Vázquez, 1987, S. 425).

In den Rahmen des 'falschen Konsens' ('false consensus'; vgl. Six, 1987, S. 130) ist die Studie von Tabachnik, Crocker und Alloy (1983) einzuordnen, die sich mit dem Urteil depressiver und nichtdepressiver Studenten befaßt, inwieweit bestimmte Attribute, teils depressionsrelevant, teils depressionsirrelevant, für die eigene Person bzw. den 'durchschnittlichen Studenten’ zutreffend erscheinen. Der Begriff des falschen Konsens bezeichnet dabei einen ,... relative effect, revealed by the difference in ratings of others between people who do and people who do not rate an attribute as true of themselves" (Crocker, Tabachnik-Kayne \& Alloy, 1985, S. 1580). Während Depressive sich als recht ausgeglichen zeigten, d. h. andere Personen entlang aller drei Dimensionen der Items als ähnlich beurteilten, schätzten sie ,... nondepressed ... DR [depressionsrelevante] items as less true of themselves and NDR [nicht depressionsrelevante] items as more true of themselves than of the average college student" (Tabachnik, Crocker \& Alloy, 1983, S. 692) ein.

Eine andere Alternative, die Selbsteinschätzung in Teilen objektiv überprüfen zu können, besteht in einem Vergleich des selbstbezogenen Urteils mit der Fremdbeurteilung einer anderen Person. Aus dem Blickwinkel der Sozialperspektiven entspricht dies einem Vergleich zwischen dem 'Autostereotyp' und dem 'Heterostereotyp' (vgl. Fassheber, Niemeyer \& Kodowski, 1990, S. 23).

Das Anliegen einer Forschungsgruppe um Lewinsohn war es, innerhalb der sozialen Interaktion die Tendenz einer Verzerrung seitens Depressiver zu finden (Lewinsohn, Mischel, Chaplin \& Barton, 1980). Angesichts der Wichtigkeit zwischenmenschlicher Prozesse für die Aufrechterhaltung einer depressiven Symptomatik versuchten sie, „to disentangle unrealistic negative self-perception from actual social deficits ...“ (Lewinsohn, Mischel, Chaplin \& Barton, 1980, S. 203). Indem sie in vier Sitzungen stationär aufgenommene Depressive, psychiatrische und normale Kontrollgruppenmitglieder behandelten und dabei deren Fremd- und Selbsteinschätzungen über verschiedene Zeitpunkte erhoben, erhielten sie 
zumindest Anhaltspunkte, um Aussagen über die defizitäre Sozialkompetenz in Abgrenzung zu einem lediglich empfundenen Mangel machen zu können.

The major and most unexpected pattern of results, however, involved the nature of the discrepancies between the original self-ratings and the initial ratings made by the observers ... . These discrepancies were significant larger for the normal and psychiatric control subjects than for the depressed individuals .... in that sense the depressed were the most realistic. (Lewinsohn, Mischel, Chaplin \& Barton, 1980, S. 210)

Bemerkenswert ist jedoch nicht nur dieses, für die damalige Forschungsgruppe überraschende, jedoch aus der Sicht der 'Sadder but wiser'-Forschung keinerlei Grund zur Verwunderung bietende Resultat, sondern auch der Befund, daß diese Realitätsorientierung im Verlauf der Intervention abnahm; die Depressiven näherten sich im Verlauf der Therapie der Überschätzung der Nichtdepressiven an (a. a. O., S. 206 ff.).

Selbstaufmerksamkeit ('self-awareness') als Mediator des depressiven Realismus war der Ansatz, der von einem mit Gibbons assoziierten Kollegenkreis eingeführt wurde (Gibbons, Ingram, Smith, Pearce \& Brehm, 1985). Dieser testete mit verschiedenen klinischen Gruppen die Wirkung der Selbstfokussierung. Neben der Beobachtung einer sich mit zunehmender Selbstaufmerksamkeit verschlechternden Stimmung - die untersuchten Personen der Experimentalgruppe mußten sich, einem Spiegel zugewandt, beschreiben - zeigte sich in deutlicher Parallelität zur depressiven Symptomatik, ,... that self-focused attention enhanced the validity of subjects' self-reports ...“ (Gibbons, Ingram, Smith, Pearce \& Brehm, 1985, S. 671). Aus diesen beiden Ergebnissen leiteten die Autoren die Vermutung ab, ,... that depressed persons do tend to be chronically self-focused“ (a. a. O., S. 673).

Strack und Coyne (1983) versuchten, ausschließlich die Informationen, die typischerweise für Depressive zugänglich sind, in ihre Studie einzubeziehen. Wie Layne (1983) ausführt, spiegelt die von Depressiven empfundene Rückweisung in der Umwelt nicht zwingend eine kognitive Verzerrung wider, sondern möglicherweise eine in der sozialen Interaktion tatsächlich unterschiedliche Haltung anderer Menschen gegenüber Depressiven im Vergleich zu Nichtdepressiven.

Um dieser Variablenkonfundierung zu entgehen, rekrutierten Strack und Coyne (1983) Personen aus einer nicht klinisch auffälligen Population mit einer depressiven Verstimmung mit der Fragestellung, ob sie ,... are able to elicit an affective reaction from others that is 
consistent with their negative self-perceptions“ (Strack \& Coyne, 1983, S. 799). Die Befunde der Studie bestätigten die Sichtweise der Depressiven, die in den fünfzehnminütigen Konversationseinheiten ihren Gesprächspartnern Gefühle der Feindseligkeit, Depression und Angst induzierten und im gleichen Maße zurückgewiesen wurden, wie sie es selbst empfanden (a. a. O., S.801 ff.). Eine negativere Sicht der eigenen sozialen Situation war nicht zu beobachten (vgl. auch Siegel \& Alloy, 1990). In der Untersuchung von Zimmergenossen eines College fanden sie ,considerable support to the view that negative social relationships occur between mildly depressed persons and significant others ..." (Siegel \& Alloy, 1990, S. 367 f.). Bei einem Vergleich der sozialen Einschätzungen lagen die Depressiven näher an der Fremdeinschätzung, während die Nichtdepressiven ihre Tendenz der Selbstüberschätzung ein weiteres Mal bekräftigten (a. a. O., S. 370).

\subsubsection{Diskussion des depressiven Realismus}

Die Verzerrung, die bei Nichtdepressiven gefunden wurde, enthält auch positive Aspekte. Sie hat Einfluß auf die phys sche und psychische Gesundheit.

Hiermit wird der Überblick abgeschlossen, der die Existenz des depressiven Realismus anhand Untersuchungen in verschiedenen Bereichen belegt. Eine Zusammenfassung der referierten Befunde, Implikationen und theoretischen Überlegungen ist an dieser Stelle nicht angebracht, da die Komplexität, die in der Befundlage zu beobachten ist, nicht durch eine Reduktion auf wenige Sätze nivelliert werden soll. Es sollen, nachdem auch positive Aspekte der kognitiven Verzerrung nichtdepressiver Personen herausgestellt werden (vgl. auch Kap. 5, S. 171), zum einen Erklärungsansätze für dieses Phänomen dargestellt werden, zum anderen Faktoren verdeutlicht werden, die für eine Einschränkung der generellen Gültigkeit der Realismushypothese wichtig sind.

The optimist thinks that this may be one of the best days ever. The pessimist fears that the optimist may be right. (Scheier \& Carver, 1992, S. 201)

Die positive Wirkung ist auf die Neigung der Nichtdepressiven zurückzuführen, der 'illusion of control' anheimzufallen (vgl. auch Scheier \& Carver, 1985). Es scheint, daß ,,... such biases and illusions enable individuals to maintain positive affect, self-regard, hope, and behavioral persistence in the face of life's obstacles and stresses and ... reduce susceptibility to depression and other illnesses“ (Alloy \& Clements, 1992, S. 241; vgl. auch S. 104). Es ist zu vermuten, daß nicht eine realistische Sicht, sondern die positive Verzerrung an der psychischen und physischen Gesundheit beteiligt ist (vgl. Kap. 2.3.2.2.2, S. 92), die 
durch bestimmte Faktoren auch über die Effekte des Selbstwertgefühls hinaus (vgl. S. 127) konstituiert wird. „Health psychologists are slowly discovering what many lay people seem to have known for years - that positive thinking ist helpful“" (Scheier \& Carver, 1992, S. 201). In Anlehnung an Motivationstheorien, die in der Psychologie zahlreich publiziert wurden und menschliches Handeln als eine Funktion von antizipierten Folgen der jeweiligen Handlungen verstehen (Weiner, 1988), wird auch in bezug auf den 'autonomen Organismus' angenommen, daß dieser in der Erwartung günstiger Folgen so 'handelt', daß die Auftretenswahrscheinlichkeit dieser Konsequenzen erhöht wird. In einer Literaturübersicht fanden Scheier und Carver (1992) überzeugende Hinweise sowohl für das psychische als auch für das physische Wohlergehen als Folge einer optimistischen Grundhaltung. Es konnte gezeigt werden, daß bei schwangeren Frauen der Grad des Optimismus zur Zeit der Einlieferung in ein Krankenhaus ein guter Prädiktor für den Grad der Depression drei Wochen nach der Geburt war. Entsprechendes konnte in einem ähnlichen Design auch bei Patienten vor bzw. nach einer Bypass-Operation beobachtet werden. Bei Frauen, die wegen Brustkrebses im ersten bzw. zweiten Stadium operiert, anschließend regelmäßig beobachtet und in diesem Rahmen auch mit der psychologischen Fragestellung untersucht wurden, konnte festgestellt werden, daß das Ausmaß des Optimismus vor der Operation in einem negativen Zusammenhang mit dem Grad der Sorgen ('distress') im Anschluß an diese stand. Im körperlichen Bereich konnten nach Operationen Verbindungen zwischen dem Grad des Optimismus und der Rehabilitationszeit, der Form des EKG, biochemischen Parametern und einer insgesamt vollständigeren Wiedereingliederung in das Sozial- und Erwerbsleben nachgewiesen werden (vgl. Scheier \& Carver, 1992; siehe auch Rosemeier, 1994).

Möglicherweise ist der Zusammenhang zwischen Optimismus und physischem bzw. psychischem Wohlergehen durch das Vorhandensein eines dritten Faktors begründbar. Diskutiert werden Bewältigungsstile ('coping') als „,... vorwiegend kognitive Strategien der Auseinandersetzung mit Stressoren und belastenden Situationen“ (Dorsch, 1992, S. 121). Optimisten haben eine Disposition zu einem problembezogenen, aktiven und planvollen Copingverhalten, während bei pessimistischen Menschen eher eine Tendenz zur Entmutigung und Aufgabe angesichts von Problemen beobachtet wurde. Des weiteren akzeptieren optimistische Menschen die Realität und nehmen Schwierigkeiten als Herausforderungen an, pessimistischere Personen dagegen versuchen eher diese Realität zu leugnen. Aus der Sicht des depressiven Realismus entsteht so ein verändertes Bild der Depression: 
Depressed individuals may be suffering from the absence or breakdown of normal optimistic biases and distortions. Maladaptive symptoms of depression, such as low self-esteem, social skills deficits, negative affect, decreased persistence, poor coping with stress, and suicidal thoughts and attempts, may be consequences, in part, of the absence of healthy personal illusions. (Alloy \& Abramson, 1988, S. 257) 


\subsection{Erklärungsansätze des depressiven Realismus}

Depressive unterscheiden sich von Nichtdepressiven nicht durch die unterschiedlichen Verarbeitungsweisen, sondern durch die Anzahl der negativen Schemata. Depressive verzichten auf den 'self-serving bias' als selbstwerterhaltende Strtegie und sind so realistischer.

Von zahlreichen Ansätzen, die sich um eine Erklärung des depressiven Realismus bemühen, werden einige im folgenden ausgeführt. Insgesamt muß aber auch hier von einem Zusammenspiel vieler Faktoren anstatt von einer Monokausalität ausgegangen werden.

In Verbindung mit Theorien, die sich der Schemata als konstituierende Elemente bedienen, liegt die Vermutung nahe, daß diese - umschrieben nach Markus (1977) als „... cognitive generalizations about the self, derived from past experience, that organize and guide the processing of self-related information contained in the individual's social experiences“ (S. 64) - an dem Prozeß der Verzerrung beteiligt sind. Diese Vorstellung entspricht dem Ansatz von Dykman, Abramson, Alloy \& Hartlage (1989), die sich gegen Unterschiede in einer 'charakteristischen' Verarbeitungsart aussprachen, indem sie auf die Möglichkeit hinwiesen, daß Depressive eventuell lediglich mehr Schemata negativen Inhalts besitzen, so daß die Wahrscheinlichkeit, neue Informationen in negative Schemata zu integrieren, allein schon durch ihre Anzahl ansteigt (vgl. auch Ackermann \& DeRubeis, 1991, S. 579). Des weiteren resultiert die positive Verzerrung möglicherweise aus positiven und sehr wirksamen Schemata Nichtdepressiver, während sich die Schemata depressiver Personen differenzierter, positive und negative Inhalte umfassend, darstellen (S. 75). Empirische Unterstützung findet diese Sicht in den Untersuchungen der Forschergruppe um Alloy (Alloy, Allbright, Abramson \& Dykman, 1990; vgl. auch S. 119).

Gemäß einer sozialpsychologischen Position entsteht der Selbstwert aus dem Vergleich mit anderen (Taylor, 1983, S. 1164 ff.; vgl. auch S. 99). Somit dient der 'self-serving bias' dem Selbstwerterhalt, der als isolierter Effekt empirisch auch in einer Studie von Koenig, Clements und Alloy (1992) verdeutlicht wurde. Sie stellten einen Zusammenhang zwischen der Höhe der illusorischen Kontrolleinschätzung (bei tatsächlicher Nichtkontingenz) und einem anschließenden Anstieg des Selbstwerts fest (S. 245). Depressive, die „,... suffer from a breakdown in their motivation to maintain self-esteem“ (Tennen \& Herzberger, 1987, S. 72; S. 115) bzw. unter einem Gefühl geringeren Selbstwerts (vgl. Beck, Rush, Shaw \& Emery, 1994), zeigen diese Verzerrung nicht und sind dadurch - in nahezu tragisch zu bezeichnender Konsequenz - realistischer. Einen weiteren Grund führen Miller und Moretti (1988) an, die Unterschiede zwischen Depressiven und Nichtdepressiven ,... in their un- 
derstanding of how causal attributions can serve the self and its needs" (S. 282) begründet sehen.

Angesichts der steigernden Wirkung der Selbstfokussierung auf den Realitätssinn (vgl. Gibbons, Ingram, Smith, Pearce \& Brehm, 1985; siehe auch S. 123) gewinnt die Überlegung an Bedeutung, den depressiven Realismus mit der Theorie der selbstgerichteten Aufmerksamkeit ('self-directed attention'29; Carver \& Scheier, 1981; vgl. auch Duval \& Wicklund, 1972) in Verbindung zu bringen; ,... when attention is directed internally, it tends to focus on whatever aspect of the self is most salient at the time“ (Gibbons \& Gaeddert, 1984, S. 162), so daß im Rahmen der geforderten Selbstberichte (siehe S. 123) die Aufmerksamkeit auf die kognitive Repräsentation der Facetten des Selbst in einem Maße gerichtet wurde, daß die Genauigkeit zunehmen konnte. Wichtig wäre demnach die Erforschung des Zusammenhangs von Depression und Selbstaufmerksamkeit. In diesem Rahmen könnte die Vermutung an Bedeutung gewinnen, ,... that depressed persons do tend to be chronically self-focused“ (Gibbons, Ingram, Smith, Pearce \& Brehm, 1985, S. 673).

\subsection{Grenzen und einschränkende Faktoren des depressiven Realismus}

Die Einschränkungen des Gültigkeitsbereichs werden aufgezeigt. Die Schwere der Depression wurde in vielen Studien nicht ausreichend beachtet. Die Wirkung der Depression und die der Mediatorvariable des Selbstwerts werden in verschidenen Untersuchungen konfundiert. Einige Veröffentlichungen widersprechen den Annahmen des depressiven Realismus.

For almost a decade now, depressive realism has been an intriguing yet poorly understood phenomenon. Whereas early work on depressive realism centered on demonstration of the realism phenomenon ..., recent work has focused on laying out its boundary conditions. (Dykman, Abramson, Alloy \& Hartlage, 1989, S. 444)

Einige Einschränkungen sind schon in den bereits referierten Studien (vgl. Kap. 2.3.2.3, S. 93 ff.) angeführt worden; hierzu gehören die Unterscheidungen, ob

- Aussagen für die eigene oder eine andere Person gemacht wurden,

- Handlungen allein oder unter Anwesenheit einer anderen Person ausgeführt wurden,

- Rückmeldungen ein- oder mehrdeutig waren,

\footnotetext{
29 „'Self-directed attention' refers to the proportion of time that one's attention is directed toward some as-
} pect of the self rather than toward the environment" (Alloy \& Abramson, 1988, S. 251). 
- Informationen sozialen Bezug hatten oder

- Rückmeldungen sofort bzw. verzögert gegeben wurden.

Hinzu kommen zwei intrinsische Faktoren, die Schwere der Depression und das Selbstkonzept des Depressiven (vgl. Alloy \& Abramson, 1988).

Benassi und Mahler (1985; vgl. auch S. 98) stießen, ähnlich wie Sacco und Hokanson (1982), auf inverse Ergebnisse bezüglich der Dimension der Rückmeldung und der des sozialen Bezugs. Während in der Situation, in der die Versuchsperson allein war, die für die 'depressive realism hypothesis' typischen Befunde repliziert werden konnten, waren die Nichtdepressiven in der Versuchsbedingung, in der eine andere Person anwesend war, in ihrer Einschätzung genauer.

In bezug auf die Rückmeldungen zeichnet sich die Tendenz ab, daß Nichtdepressive sowohl auf unmittelbare als auch auf verzögerte Rückmeldungen mit einer Verzerrung reagieren, wohingegen bei Depressiven lediglich in der Verzögerungsbedingung eine negative Tendenz zu beobachten war. Wurde die Rückmeldung sofort gegeben, waren sie in ihrer Einschätzung realistischer (vgl. Kap. 0, S. 116). Diese Beobachtung könnte ein Anzeichen für unterschiedliche Ansatzpunkte der Verzerrung im Verarbeitungsprozeß sein. Der der Verzerrung zugrundeliegende Mechanismus tritt bei Nichtdepressiven früher in Kraft als bei Depressiven.

Die Variation des Bezugspunkts zeigt eine große Wirkung auf das abzugebende Urteil. Die für die eigene Person abgegebenen Einschätzungen folgen dem vielzitierten Trend des depressiven Realismus, die für eine andere Person abgegebenen Urteile dem entgegengesetzten. Nichtdepressive schätzen die Kontrolle für andere Personen realistisch ein, Depressive verfallen der illusionären Kontrollüberzeugung (vgl. Kap. 0, S. 116).

Der Grad der Depressivität stellt einen weiteren möglichen Einflußfaktor dar. Die Befunde des 'depressive realism' widersprechen denen der klinischen Praxis. Da es sich aber um ein stabiles Phänomen handelt (vgl. Mark \& Stevens, 1988, S. 353 f.), kann dieser Widerspruch durch die Annahme aufgelöst werden, daß die Genauigkeit der Einschätzungen in Abhängigkeit vom Grad der Depressivität mit einer umgekehrten U-Funktion beschreibbar ist. Depressive bzw. Nichtdepressive weisen somit eine negative bzw. positive Verzerrung auf, während nur Personen mit leichten Depressionen in der Mitte der zugrundegelegten Funktion einen angemessenen Realitätssinn beweisen. 
Gegen diese Annahmen sprechen Befunde von Loewenstein und Hokanson (1986; vgl. auch S. 121), die weder bei leichten noch bei schweren Depressionen eine Verzerrung fanden, sowie die theoretische Überlegung von Mark und Stevens (1988), die - dem Paradigma der kognitiven Theorien verpflichtet - anmerken, daß ,... some evidence to suggest that severely depressed persons differ cognitively from mildly depressed persons in evidencing the negative thinking errors hypothesized by cognitive intervention theorists, this evidence is hardly conclusive“"(1988, S. 354).

Ruehlmann, West und Pasahow (1985), die in einer Literaturübersicht den Einfluß des Depressionsgrades nicht verneinen, bleiben jedoch in ihrer abschließenden Beurteilung zurückhaltend. Unterschiede, so die Autoren, die aus dem Vergleich von leichten und schweren Depressionen resultieren, können noch eine weitere Grundlage haben. In Studien, die sich mit Versuchspersonen mit geringen Depressionswerten befaßten, wurden fast immer Studenten untersucht, während Probanden mit schwereren Depressionen zumeist Patienten außerhalb des universitären Kontextes waren. Die gefundenen Differenzen können somit auch auf die Unterschiedlichkeit der Populationen zurückgeführt werden (vgl. Ruehlmann, West \& Pasahow, 1985, S. 60).

Abschließend soll die Einflußmöglichkeit des Selbstwerts erwähnt werden. Wie schon bei der Besprechung der Studie von Tennen und Herzberger (1987; siehe auch S. 115) angeführt, handelt es sich bei der ausgeglicheneren Art der Attribuierung bei Depressiven eventuell nicht um eine Wirkung der Depression als solche, sondern um einen Einfluß, der auf die Mediatorvariable des Selbstwerts zurückzuführen ist. Diese Vermutung steht mit der Beobachtung in Übereinstimmung, daß in der erwähnten Studie ausgeglichene Attribution stärker mit dem Selbstwert als mit dem Grad der Depressivität assoziiert war.

In der Fachliteratur sind jedoch auch Studien veröffentlicht worden, deren Befunde die Theorie des depressiven Realismus nicht stützen. Mit dem Ziel, ein aussagekräftigeres und damit kritischeres (im Sinne Poppers; vgl. Westermann, 1987, S. 13 ff.) Testverfahren zur Überprüfung der 'Sadder but wiser'-Hypothese anzuwenden, modifizierten Bryson, Doan und Pasquali (1984) das zweite Experiment von Alloy und Abramson (1979, S. 457 ff.; vgl. auch S. 95), da dieses ,,... the only one showing a mood effect where winning or losing money was not part of the manipulation“ (Bryson, Doan und Pasquali, 1984, S. 109) sei. Zusätzlich zu der ursprünglichen experimentellen Anordnung (vgl. S. 94) wurde versucht, Selbstattributionsfehler der Depressiven in bezug auf Inkompetenz aus der Interpretation der experimentellen Handlung herauszufiltern (ebd.). Die Versuchspersonen mußten einschätzen, wie häufig sie die Möglichkeit hatten, durch Knopfdruck das 'grüne Licht' zum 
Aufleuchten zu bringen, wie häufig sie dies tatsächlich taten (im Verhältnis zu dem, was möglich gewesen wäre) und wie häufig das zufallsgesteuerte Aufleuchten vorkam. Werden die Nichtdepressiven von einer Tendenz geleitet, sich selbst in positiverem Licht zu sehen, müßte sich ihr Urteil im Anschluß an das Experiment darüber, wie oft sie die experimentelle Reaktion hervorriefen, der Einschätzung annähern, wie oft dies möglich gewesen wäre. Die Ergebnisse sind aus dem Blickwinkel der 'depressive realism hypothesis' desillusionierend; ,... we found no evidence that judgments of control vary as a function of problem type, mood, sex, or any combination thereof" (Bryson, Doan und Pasquali, 1984, S. 113). Lediglich die Einschätzung der potentiellen Kontrolle über das Aufleuchten des Lichts im Verhältnis zu der tatsächlichen Häufigkeit variierte in Abhängigkeit von der Frequenz, ohne aber dabei Unterschiede zwischen den beiden Versuchsgruppen hervorzubringen. In der '75-75'-Bedingung ${ }^{30}$ gaben die Versuchspersonen an, das Aufleuchten des Lichts entsprechend der Möglichkeiten hervorgerufen zu haben, und daß es unter einer Zufallsbedingung weit weniger häufig aufgeleuchtet wäre; in der '25-25'-Bedingung herrschte der Eindruck vor, das experimentelle Ergebnis häufiger provoziert haben zu können, und dies schon allein, wenn sie ,... decided to press or not press on each trial by flipping a coin“ (Bryson, Doan \& Pasquali, 1984, S. 114; siehe auch: Campbell \& Fehr, 1990; Dunning \& Story, 1991; Dykman, Abramson, Alloy \& Hartlage, 1989; Gotlib, 1981; Gotlib, 1983; HoehnHyde, Schlottman \& Rush, 1982; Janning, Klingberg \& Engberding, 1993; Oliver, Raftery, Reeb \& Delany, 1993; Werner \& Rehm, 1975).

In der Methodik an die Studie von Alloy und Abramson (1979) angelehnt, urteilten von Basewitz, van Gülick, Oettinger und Hautzinger (1989) im Anschluß an ihre Untersuchung:

Kontingenz und Depression scheinen zumindest in der reduzierten Welt der Schalter und Signale, unter Umständen mit Pfennigbeträgen angereichert[,] keine stringent verknüpften Phänomene zu sein, wie die Theorie der Erlernten Hilflosigkeit postuliert. Das Resümee von Alloy und Abramson (1979), Depressive seien 'sadder but wiser', konnte nicht repliziert werden. (Von Basewitz, van Gülick, Oettinger \& Hautzinger, 1989, S. 97)

\footnotetext{
${ }^{30}$ Sowohl das Drücken als auch das Nichtdrücken des Knopfs wird experimentell mit dem Aufleuchten des grünen Lichts in $75 \%$ der Fälle beantwortet, es besteht also bei hoher Frequenz des experimentellen 'outcome' keine Kontingenz.
} 


\subsection{Ableitung der sportpsychologischen Hypothesen}

Vor dem Hintergrund der referierten Ansätze und Befunde werden die sportpsychologischen Hypothesen abgeleitet. Die Haupthypothesen (SPH 1 und SPH 4) gehen bei der Vorhersage der eigenen Leistungssteigerung bzw. der durch die Bedstungswahrnehmung vermittelten Selbsteinschätzung von einem Wert aus, der seitens der Depressiven in größerer Übeeinstimmung mit dem der tatsächlichen Leistungssteigerung steht.

Hiermit sind der theoretische und der empirische Hintergrund abgeschlossen, die sich mit der Schädigung von ZNS-Strukturen und deren Implikationen, der motorischen Wiederherstellung und der Belastungswahrnehmung auf der einen, dem Phänomen des depressiven Realismus auf der anderen Seite beschäftigten.

Ausgehend von diesen Ansätzen und Befunden leitet sich die Vermutung ab, daß auch hinsichtlich der Selbsteinschätzung, die sich auf die Wiederherstellung der eigenen körperlichen Funktionen bezieht, eine systematische Variation entlang des Grades der Depression zu beobachten ist. Zugleich wird diesem Bereich mehr persönliche Relevanz zugerechnet als der ,... reduzierten Welt der Schalter und Signale, [die] unter Umständen mit Pfennigbeträgen angereichert“ (Von Basewitz, van Gülick, Oettinger \& Hautzinger, 1989, S. 97; vgl. Blockzitat zur Methodenkritik, S. 131) wurde.

Den zweiten Schwerpunkt bilden die Belastungswahrnehmung und die Einschätzung der eigenen Kraft. Auch bei dieser gilt die Annahme, daß die Übereinstimmung der durch das Einschätzen der halben Maximalkraft vorgenommenen 'Aussage' zu der tatsächlichen Maximalkraft in Abhängigkeit vom Grad der Depressivität variiert. Das eine Mal handelt es sich somit um eine verbale Vorhersage, das andere Mal um eine nonverbale 'Vorhersage', die durch eine Handlung gemacht wird. In beiden Fällen besteht die Möglichkeit der realitätsnahen Einschätzung sowie der Über- und der Unterschätzung ${ }^{31}$.

Hieraus leitet sich die erste sportpsychologische Hypothese ab, nach der bezüglich der Vorhersage der eigenen Leistungssteigerung ein Wert erwartet wird, der seitens der Depressiven in größerer Übereinstimmung mit dem der tatsächlichen Leistungssteigerung steht. Hiernach wären die Depressiven in ihrer Zukunftserwartung nicht negativ verzerrt, sondern realistischer. Die zweite sportpsychologische Hypothese, die in Verbindung mit der dritten die Rahmenbedingungen der ersten Hypothese näher beleuchten soll, besagt, daß Depressive insgesamt einen geringeren Wert vorhersagen. Mit ihr wird gleichzeitig die klassische Sicht der Depression überprüft, die von einer negativeren Zukunftserwartung

\footnotetext{
${ }^{31}$ In der Auswertung der zentralen Hypothesen $(\mathrm{H} 1$ und $\mathrm{H}$ 4) wird der Unterschied zwischen Unter- und Überschätzung jedoch nicht berücksichtigt. Es werden alle Werte in positive umgewandelt, um zu erreichen, daß zum Zweck einer strengeren Hypothesenprüfung ein kleinerer Wert (d. h. weniger Abweichung) immer realitätsnäher ist als ein größerer (vgl. S. 139 sowie Kap. 5, S. 171).
} 
ausgeht (vgl. auch S. 76). Sie ist insofern auch mit dem Hauptziel der vorliegenden Arbeit vereinbar, als sie nicht die niedrigere Erwartung im Vergleich zu Nichtdepressiven in Frage stellt, sondern im Vergleich zur Realität. Ein Unterschied zwischen den beiden Gruppen wird sowohl von den Thesen der vorliegenden Arbeit als auch von den klassischen Depressionstheorien vertreten, doch an der Realität objektiviert schätzt sich die eine Gruppe realistischer ein, während die andere sich über- bzw. unterschätzt. Gemäß der dritten sportpsychologischen Hypothese haben Nichtdepressive auch tatsächliche höhere Leistungssteigerungen zu verzeichnen. Die vierte sportpsychologische Hypothese steht in Parallelität zu der ersten; sie ist die zentrale Hypothese der Belastungswahrnehmung, die von einem größerem Realismus der Depressiven im Vorhersagen der Maximalkraft durch Drücken der Hälfte dieser Maximalkraft ausgeht.

Den konzeptuellen Kern der Arbeit bilden die erste und die vierte Hypothese. Mit der zweiten und dritten Hypothese können zusätzliche Aussagen gemacht bzw. die Rahmenbedingungen der ersten Haupthypothese (erste Hypothese) näher umrissen werden. In bezug auf die zweite Haupthypothese (vierte Hypothese) werden keine zusätzlichen Ableitungen gemacht, da in der 'Vorhersage' durch das Drücken der halben Kraft 'Vorhersage' (zweite Hypothese) und Bezug zu der tatsächlichen Leistung (dritte Hypothese) konfundieren. Die 'Vorhersage' und die Abweichung von dem aus der halbierten Maximalkraft errechneten Referenzwert sind identisch, so daß weitere Hypothesen hier keinen zusätzlichen Nutzen bringen könnten. Bei der verbalen Vorhersage der Leistungssteigerung variieren der tatsächliche Leistungszuwachs und die Vorhersage per se unabhängig von der Haupthypothese, so daß die zusätzlichen Hypothesen (zweite und dritte Hypothese) in diesem Kontext nützlich sind.

Die sportpsychologischen Hypothesen (SPH) im Überblick lauten (die zentralen Hypothesen sind durch Pfeile hervorgehoben):

$\Rightarrow$ SPH 1: Depressive schätzen sich hinsichtlich der Genauigkeit der Vorhersage der Leistungssteigerung realistischer ein.

- SPH 2: Die Höhe der vorhergesagten Leistungssteigerung ist bei Depressiven geringer.

- SPH 3: Depressive zeigen eine geringere Leistungssteigerung.

$\Rightarrow$ SPH 4: Depressive sind hinsichtlich der durch die Belastungswahrnehmung vermittelten Selbsteinschätzung realistischer. 


\section{Methode}

\subsection{Versuchsplanung, Vorhersagen und statistische Hypothese}

Mit dem Design einer Korrelationsstudie wird ein Versuchsplan implementiert, in dem bei den zentralen Hypothesen (SPH 1 und SPH 4) die Genauigkeit der Vorhersage bzw. der Einschätzung die Kriteriumsvariable ist, die mit dem Depressionsgrad, der als Prädiktorvariable fungiert, korreliert wird; die Erwartung hierbei ist, daß die Genauigkeit der Vorhersage bzw. der Einschätzung in Abhängigkeit vom Grad der Depressivität zunimmt. Die Einteilung in Kriteriums- und Prädiktorvariable entspricht der Einteilung der Variablen in abhängige und unabhängige Variablen, „,... wenngleich durch letztere Bezeichnung eine engere, gerichtete Kausalbeziehung zum Ausdruck gebracht wird. ... Prädiktorvariablen sind i. a. einfacher und billiger meßbar und können - im Kontext von Vorhersage im eigentlichen Wortsinne - zu einem früheren Zeitpunkt als die eigentlich interessierenden Kriteriumsvariablen erfaßt werden. Typische Prädiktorvariablen sind psychologische oder medizinische Tests...“ (Bortz, 1989, S. 214). Gleichzeitig gelingt es auf diese Weise, der Empfehlung W. L. Hays zu folgen, die in Abwandlung nach Hager (1987) nahelegt, ,... den einfachsten verfügbaren Versuchsplan, auf dessen Grundlage eine valide Hypothesenprüfung erfolgen kann“ (S. 238), zu wählen.

Die Operationalisierung der Genauigkeit der Vorhersage bzw. der Selbsteinschätzung, die die Kriteriumsvariablen der zentralen Hypothesen (SPH 1 und SPH 4) bilden, erfolgt über die Abweichung des verbal bzw. nonverbal 'vorhergesagten' Werts von dem der tatsächlichen Leistungssteigerung bzw. Leistung (für die rechnerische Umsetzung dieser Operationalisierung siehe S. 139). Die Kriteriumsvariable der zweiten sportpsychologischen Hypothese (SPH 2) bildet die Höhe der vorhergesagten Leistungssteigerung, die der dritten Hypothese (SPH 3) die Höhe der tatsächlichen Leistungssteigerung (siehe auch S. 139).

Im Gegensatz zu allgemeinen Validisierungen in der neurologischen Rehabilitation, die „... sich vor allem auf die allgemeingültigen Erfordernisse des täglichen Lebens, die sogenannten 'activities of daily living' konzentrieren ... [, erhoben beispielsweise durch den] Barthel Index (BI)“ (Hesse \& Mauritz, 1994, S. 56), erfolgt die Operationalisierung der motorischen Leistungssteigerung als Teilprozeß der motorischen Funktionswiederherstellung in der vorliegenden Arbeit anhand der in der Sportwissenschaft üblichen Einteilung der motorischen Fähigkeit in koordinative und konditionelle Eigenschaften, wobei die konditionellen wiederum Ausdauer, Kraft und Schnelligkeit umfassen (Blume, 1981; vgl. Kap. 2.1.3.1, S. 25). 
Die Kraft wird über die Handschlußkraft operationalisiert, die mit einem Handdynamometer ermittelt wird (Mathiowetz, Kashman, Vollan, Weber, Dowe \& Rogers, 1985; Robinson, Fitts \& Kraft, 1990; für eine detaillierte Beschreibung der einzelnen Verfahren s. u., S. 143 ff.). Zur Erhebung der Genauigkeit der Selbsteinschätzung, vermittelt durch die Belastungswahrnehmung, wird zuvor die Kraft gemessen, die die Versuchsperson durch das Zusammendrücken des Gerätegriffs als genau die Hälfte der antizipierten eigenen Maximalkraft 'vorhersagt'. Im Gegensatz zum üblichen Vorgehen in der Forschung der Belastungswahrnehmung bzw. der psychophysikalischen Skalierung (vgl. Kap. 2.2.2.2, S. 58), in der zumeist Standardreize, Kategorien oder Verhältnisse vorgegeben werden, auf die bezugnehmend andere Reize (Gewicht, Lautstärke, Helligkeit) oder Reizausprägungen eingeschätzt werden, gilt es hier, eine zuvor theoretische, nicht erfahrene Größe einzuschätzen. Im Rahmen der vorliegenden Untersuchung ist es die Hälfte der eigenen maximalen Handschlußkraft, die beispielsweise durch das Gefühl der Anstrengung, der Kraftempfindung oder des an den Handflächen empfundenen Drucks vermittelt wird. Die Wahrscheinlichkeit, daß eine Versuchsperson vor kürzerer Zeit durch andere Umstände erfahren hat, wie groß die eigene Handschlußkraft ist und wie sich genau die Hälfte dieser anfühlt, ist so gering, daß sie schon im Vorfeld der theoretischen Betrachtung systematischer Einflußgrößen vernachlässigt werden kann.

Die Schnelligkeit wird - im Sinne der Tappingrate - als die Anzahl der Kontakte auf der Leer-Taste einer PC-Tastatur während eines Zeitraums von zehn Sekunden definiert. In ähnlichen Untersuchungen ,finger tapping scores were the mean of five 10-s trials with each hand" (Haaland, Temkin, Randahl \& Dikmen, 1994, S. 450). Da die Vorhersage auf der Basis des besten von drei Versuchen erhoben wird, geht in der vorliegenden Untersuchung sowohl bezüglich der Kraft als auch der Schnelligkeit nicht der Mittelwert, sondern die jeweils beste Leistung in die Wertung ein (s. u.).

Zur Messung der Kraft und der Schnelligkeit kann ergänzend gesagt werden: „Fingertapping rate (TAP) and grip strength (GRIP) are common tests for assessment of upper extremity motor control“ (Robinson, Fitts \& Kraft, 1990, S. 695), „,... are easy to administer, are widely used by clinical neuropsychologists, have a stronge normative base ... and differ in their dependence on speed. Therefore, they represent a reasonable first step in more carefully assessing motor deficits after head injury“ (Haaland, Temkin, Randahl \& Dikmen, 1994, S. 449; vgl. auch in bezug auf das Tapping: Salmoni \& McIlwain, 1979).

Die Ausdauer wird über eine adaptierte Form der PWC 170 (WHO; vgl. Neumann \& Schüler, 1994) erhoben. Hierbei wird mit einem Fahrradergometer die Leistung in Watt gemessen, bei der eine Herzfrequenz von 130 Schlägen/min als 'steady state' erreicht wird 
(Physical Working Capacity 130). Die Operationalisierung des 'steady state' ist das Einpendeln der Herzfrequenz auf 130 Schläge/min, und die relative Stabilität innerhalb dieses Werts im Verlauf von zwei Minuten auf gleicher Belastungsstufe. „Die PWC 170 als international gut eingeführte Leistungsgröße in Watt bzw. $\mathrm{kpm} / \mathrm{min}$ entspricht derjenigen Leistung auf dem Ergometer, bei welcher eine Herzfrequenz von 170 Schlägen/min erreicht wird. .... Im Rahmen jeder Leistungsdiagnostik ist die kardio-zirkulatorische Funktionsprüfung ... besonders wichtig“ (Nowacki, 1987, S. 242-244).

Die Koordination wird mit Hilfe des Programmoduls 'Visuomotorik' des Computerprogramms 'Rehacom' als visuomotorische Handkoordination (vgl. auch Labyrinth-Test; Brikkenkamp, 1975, S. 149) operationalisiert (Koordination I), bei dem mit einem Hebel ein Kreis auf dem Bildschirm bewegt wird und ein Objekt verfolgt werden soll; gemessen wird dabei die Fehlerzeit (Objekt ist nicht innerhalb des Kreises) in Prozent (vgl. Weber, Regel \& Krause, 1995). Des weiteren wird die Koordination - auch als Handkoordination, aber in Verbindung mit einer psychomotorischen Geschwindigkeitskomponente - mit Hilfe eines Versuchsteils, in dem drei Schrauben in ein Schraubenbrett gedreht werden müssen, operationalisiert (Koordination II). Abhängige Variable ist hierbei die benötigte Zeit, die ab Aufnehmen der ersten Schraube gemessen wird (vgl. Goldenberg, 1995).

Die Operationalisierung des Depressionswerts - der die Prädiktorvariable bildet - erfolgt über das Depressionsinventar von Beck (BDI; Hautzinger, Bailer, Worall, Keller \& Beck, 1994; vgl. Kap. 3.3.2.6. S. 145; s. auch Anhang B, S. 221 f.). 


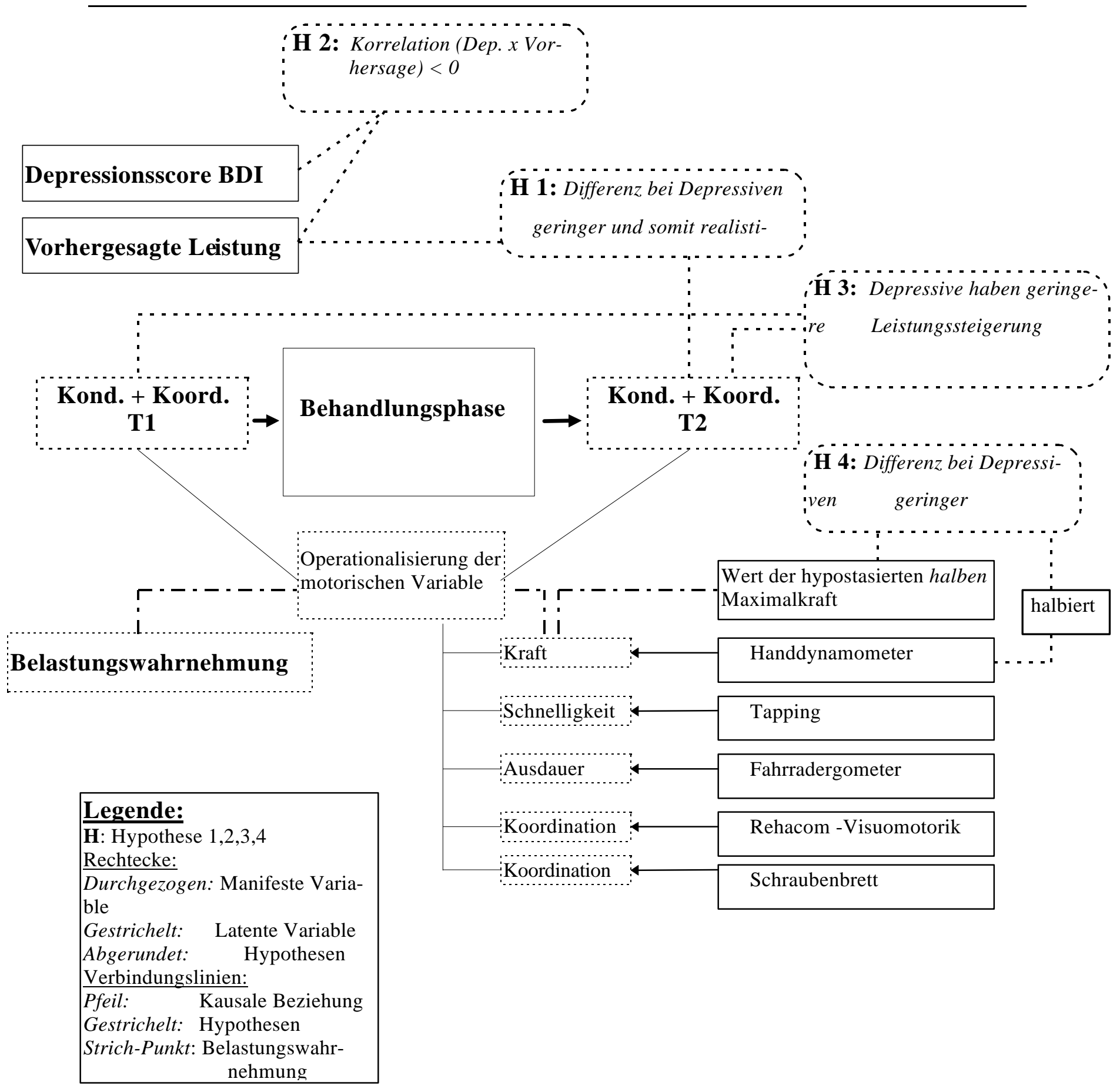

Abbildung 2: Flußschema des Versuchsplans

$\mathrm{Zu}$ Beginn und am Ende des Rehabilitationsaufenthaltes werden zwei Untersuchungen durchgeführt. Zum Zeitpunkt der ersten Untersuchung werden zusätzlich der Depressionsgrad, die auf jeden Subtest bezogene erwartete Leistungssteigerung und die Selbsteinschätzung, vermittelt durch die Belastungswahrnehmung (SPH4), erhoben. Aufgrund der sportpsychologischen Hypothese wird erwartet, daß die Übereinstimmung der vorhergesagten Leistungssteigerung mit der tatsächlichen in Abhängigkeit vom Grad der Depressivität 
zunimmt (SPH 1). Die Absolutwerte (positiv oder negativ) müssen sich daher mit steigendem Depressionswert dem Wert 'Null' annähern. Nichtdepressive erwarten mehr Leistungssteigerung als Depressive (SPH 2), aber auch mehr, als es der tatsächlichen Zunahme entspricht (SPH 1). Des weiteren wird überprüft, ob die Depressiven (möglicherweise aufgrund ihrer niedrigeren Vorhersagen) auch tatsächlich eine geringere Leistungssteigerung zu erwarten haben (SPH 3). Die vierte sportpsychologische Hypothese besagt, daß sich in der Belastungswahrnehmung die Depressiven genauer einschätzen, also in der 'HalbenKraft-Bedingung' mit dem anschließend erhobenen und halbierten Maximalwert eine größere Übereinstimmung zeigen (SPH 4).

Die sportpsychologischen Vorhersagen(SPV) lauten dementsprechend:

$\Rightarrow$ SPV 1: Die Differenz zwischen der zum Zeitpunkt $t_{1}$ vorhergesagten Leistungssteigerung in Prozent und der tatsächlichen Leistungssteigerung in Prozent zum Zeitpunkt $\mathrm{t}_{2}$ nimmt mit zunehmendem Grad der Depressivität ab.

- SPV 2: Die Höhe der vorhergesagten Leistungssteigerung in Prozent zum Zeitpunkt $\mathrm{t}_{1}$ steht im reziproken Verhältnis zum Depressionswert.

- SPV 3: Die Differenz zwischen der Leistung zum Zeitpunkt $t_{1}$ und der zum Zeitpunkt $t_{2}$ nimmt mit zunehmendem Grad der Depressivität $a b$.

$\Rightarrow$ SPV 4: Der prozentualisierte Wert der Abweichung des in der 'Halben-KraftBedingung' der Belastungswahrnehmungsbedingung ermittelten Werts von dem (anschließend erhobenen und dann) halbierten Maximalwert nimmt mit zunehmendem Grad der Depressivität ab.

Die statistische Signifikanzprüfung erfolgt über einen t-Test für unabhängige Stichproben $^{32}$ auf dem $5 \%$-Niveau $(\alpha=.05)$.

Die zu den sportpsychologischen Vorhersagen analogen statistischen Hypothesen (SH) lauten:

\footnotetext{
${ }^{32}$ Nach Bortz (1989) ist der t-Test für abhängige Stichproben auch dann zu verwenden, ,... wenn an einer Stichprobe zwei Messungen durchgeführt werden (Meßwiederholung)“ (S. 170; Hervorhebungen im Original). Die Begründung hierfür liegt in dem Umstand, daß sonst „,.. Unterschiede zwischen den Personen, die vor und nach [beispielsweise einer] ... Schulung bestehen, doppelt berücksichtigt [werden], weil sie den Standardfehler des ersten Mittelwertes und des zweiten Mittelwertes zumindest teilweise beeinflussen“ (Bortz, 1989, S. 170). Bei der vorliegenden Untersuchung handelt es sich jedoch nicht um einen Mittelwertvergleich der Untersuchungen zu zwei Zeitpunkten, da der Unterschied zwischen den zwei Zeitpunkten als ein Wert in die dann über den t-Test zu überprüfende Korrelation eingeht und so schon individuell bezogen ist, die individuellen Ausprägungen demnach nicht zweimal systematisch variieren.
} 


$\begin{array}{lll}\text { Alternativhypothesen: } & \text { SH 1: } & \mathrm{H}_{1,1}: \rho_{(\mathrm{Abw} * \mathrm{BDI})}<0 \\ \text { SH 2: } & \mathrm{H}_{1,2}: \rho_{(\mathrm{Vor} * \mathrm{BDI})}<0 \\ \text { SH 3 } & \mathrm{H}_{1,3}: \rho_{\text {(Leist*BDI) }}<0 \\ \text { SH 4: } & \mathrm{H}_{1,4}: \rho_{(\mathrm{Abwb} * \mathrm{BDI})}<0\end{array}$

Legende: BDI: Summenscore des Depressionsinventars von Beck; Abwv: Abweichung von Vorhersage und Leistung $t_{2}$ in Prozent, nur positive Werte: I ((Vorhergesagte Leistung / Leistung $\left.t_{1}\right)$ x $\left.100-100\right)$ - (Leistung $\mathrm{t}_{2}$ / Leistung $\mathrm{t}_{1}$ ) x 100 - 100)|; Vor: Vorhersage in Prozent: (Vorhergesagte Leistung / Leistung $\mathrm{t}_{1}$ ) x 100 100; Leist: Tatsächliche Leistungssteigerung in Prozent: (Leistung $t_{2} /$ Leistung $t_{1}$ ) x 100 - 100; Abwb: Abweichungen innerhalb der Belastungswahrnehmungsbedingung in Prozent: | (Kraft 'halbe Maximalkraft' | (Maximalkraft / 2)) x 100 - 100]।, wobei: / = Division, $\mathrm{x}=$ Multiplikation, - = Substraktion

Abbildung 3: Ableitung der statistischen Alternativhypothesen

„In Abhängigkeit von der Alternativhypothese, die eigentlich überprüft werden soll, wird eine konkurrierende Hypothese, die sogenannte Nullhypothese formuliert. Sie beinhaltet allgemein, daß der in der Alternativhypothese formulierte Sachverhalt nicht zutrifft, daß er sozusagen 'null und nichtig' ist .... Die statistische Nullhypothese $\left(\mathrm{H}_{0}\right)$ folgt ebenfalls zwingend aus der statistischen Alternativhypothese $\left(\mathrm{H}_{1}\right)^{\text {“ }}$ (Bortz, 1989, S. 144, Hervorhebungen im Original; vgl. auch Fußnote 41, S. 153).

\section{Nullhypothesen: $\quad$ SH 1: $\quad \mathrm{H}_{0,1}: \rho_{(\mathrm{Abwv} * \mathrm{BDI})} \geq 0$}

SH 2: $\quad \mathrm{H}_{0,2}: \rho_{(\mathrm{Vor} * \mathrm{BDI})} \geq 0$

SH $3 \quad \mathrm{H}_{0,3}: \rho_{\text {(Leist*BDI) }} \geq 0$

SH 4: $\quad \mathrm{H}_{0,4}: \rho_{(\mathrm{Abwb} * \mathrm{BDI})} \geq 0$

Legende: BDI: Summenscore des Depressionsinventars von Beck; Abwv: Abweichung von Vorhersage und Leistung $t_{2}$ in Prozent, nur positive Werte: | ((Vorhergesagte Leistung / Leistung $\left.t_{1}\right)$ x $\left.100-100\right)$ - (Leistung $\mathrm{t}_{2} /$ Leistung $\mathrm{t}_{1}$ ) x $100-100$ )|; Vor: Vorhersage in Prozent: (Vorhergesagte Leistung / Leistung $\mathrm{t}_{1}$ ) x 100 100; Leist: Tatsächliche Leistungssteigerung in Prozent: (Leistung $\mathrm{t}_{2} /$ Leistung $\mathrm{t}_{1}$ ) x 100 - 100; Abwb: Abweichungen innerhalb der Belastungswahrnehmungsbedingung in Prozent: | (Kraft 'halbe Maximalkraft' | (Maximalkraft / 2)) x 100 - 100] I, wobei: / = Division, $\mathrm{x}=$ Multiplikation, - = Substraktion

Abbildung 4: Ableitung der statistischen Nullhypothesen 


\subsection{Versuchspersonen}

Versuchspersonen waren ausschließlich Patienten der ambulanten Rehabilitationseinrichtung 'rehamed-Neuro' in Stuttgart, die innerhalb des Versuchszeitraums vom 17. Januar 1997 bis 16. Juni 1997 in dieser Einrichtung behandelt wurden. Das Einschlußkriterium war eine traumatische ZNS-Schädigung, die Ausschlußkriterien waren entzündliche/chronische/progrediente Erkrankungen, ein $\mathrm{zu}$ geringes motorisches Funktionsniveau (komplette Paresen) und neuropsychologische Defizite (einschließlich aphasischer Störungen), die das Verständnis der Versuchsanweisungen bzw. das Ausfüllen des BDI (vgl. Kap. 3.3.2.7, S. 145) beeinträchtigt hätten.

Insgesamt erfüllten 34 Patienten diese Kriterien (vgl. Anhang C, S. 223), von denen jedoch nicht jede Person an jeder Versuchsbedingung teilnehmen konnte. Im Ergebnisteil (siehe S. 152 ff.) ist jeweils die Anzahl der Patienten (n) pro Bedingung angeführt. Das Durchschnittsalter der Patienten betrug 56.06 Jahre $\left( \pm 16.48^{33}\right)$, 14 Patienten waren weiblich, 20 Patienten waren männlich. Diagnostisch handelte es sich bei 27 Personen um einen Z. n. apoplektischem Insult, bei 4 Personen um einen Z. n. Schädel-Hirn-Trauma, bei 2 Personen um einen Z. n. Shuntlegung und bei einer Patientin um einen Z. n. Tumorektomie. Der Zeitraum zwischen dem Tag der Schädigung (apoplektischer Insult, Operation mit Shuntlegung, Operation mit Tumorektomie) und dem ersten Untersuchungstermin betrug etwa $^{34} 277.91$ Tage $( \pm 319.54)$. Die linke Hemisphäre des Gehirns war in 15 Fällen betroffen, die rechte in 19 Fällen. Der durchschnittliche Depressionswert (BDI) lag bei 9.94 $( \pm 5.76)$. Der Zeitraum zwischen der ersten und der zweiten Untersuchung betrug 31.06 Tage $( \pm 11.83)$.

\footnotetext{
${ }^{33}$ Mittelwert ( \pm Standardabweichung)

${ }^{34}$ Errechnet auf der Basis von 30 Tagen/Monat und 360 Tagen/Jahr. Bei den Personen, in denen nur der Monat bekannt war, wurde der erste Tag des Monats angenommen. Wenn nur das Jahr bekannt war, wurde von dem 01.06. dieses Jahres ausgegangen.
} 


\subsection{Instruktion, Material, Hilfsmittel und Geräte}

\subsubsection{Versuchsinstruktion}

Die Versuchsinstruktionen wurden vor Beginn der Versuche von den Versuchsleiterinnen (VL) memorisiert und nach Möglichkeit (siehe Fußnote 35, S. 147) in der folgenden Weise den Patienten gegeben:

\subsubsection{Handdynamometer}

„Sie sehen hier ein Handdynamometer [HD], mit dem man die Kraft in der Hand messen kann. Halten sie ihn so (Arm hängen lassen, Hand nach unten, HD liegt seitlich in der Hand) und drücken sie (drücken lassen). ... Gut, sie merken, daß sich der Griff kaum zusammendrücken läßt, es wird aber jeder Druck genau registriert (demonstrieren, indem die VL den HD leicht zusammendrückt, während der Patient die Skala sehen kann). ... Es gilt nun, zuerst mit genau der Hälfte (betont) ihrer Kraft zu drücken. Überlegen sie, wie fest sie glauben, drücken zu können, und versuchen sie dann, mit genau der Hälfte dieser Kraft zu drücken. Wir wollen sehen, wie genau sie dies einschätzen können. ... Nun versuchen sie, so fest zu drücken, wie es nur irgendwie geht ... gut, noch einmal ... gut, und ein letztes Mal.“

\subsubsection{Tapping}

„Sie sehen hier eine Tastatur, wie man sie für Computer benutzt, wichtig für uns ist aber nur diese eine Taste (Leertaste zeigen). Bitte versuchen Sie, einige Male so schnell wie möglich diese Taste zu drücken (Arm liegt nicht auf). ... Gut. Sie sehen, wie bei jedem Anschlag dieser Kreis (zeigen) weiter nach rechts rückt. Nun geht es darum, innerhalb von zehn Sekunden so oft wie möglich diese Taste anzuschlagen, je weiter rechts der Kreis dann steht, desto besser. Ich fange an zu stoppen, wenn sie das erste Mal die Taste berühren.“

\subsubsection{Fahrradergometer}

„Bitte versuchen sie, möglichst gleichmäßig zu treten (Kontrolle, daß 60 Umdrehungen/min (RPM) in etwa eingehalten werden). Wir messen dabei ihre Herzfrequenz (Brustgurt ist angelegt), bitte haben sie keine Angst, wir werden sie nicht überbeanspruchen. Wir steigern die Belastung alle zwei Minuten ein wenig. Fahren sie ganz normal 
weiter, es wird nicht zu anstrengend. Wir wollen nur eine Frequenz von 130 Schlägen pro Minute erreichen, was völlig ungefährlich ist.“

\subsubsection{PC-Rehacom-Visuomotorik}

„Sie sehen hier eine Landschaft, die auf dem Computer nachgebaut ist. Natürlich sind die einzelnen Elemente nicht so deutlich zu erkennen, doch sehen sie hier, das ist ein Baum (zeigen), das Helle ist die Straße. Hier ist ein Auto (zeigen), das sich in unserer Geschichte auf der Flucht befindet. Sie sollen mit diesem Hebel (zeigen) diesen Kreis (zeigen) immer genau über dem Auto halten, es ist sozusagen der Suchscheinwerfer eines Hubschraubers, der das Auto verfolgt. Probieren sie es einmal (2 min Übungszeit). ... Nun wollen wir mit dem eigentlichen Versuch beginnen. Bitte versuchen sie, den Kreis immer so genau wie möglich über dem Auto zu halten. Es geht los, wenn der Kreis zum ersten Mal über dem Auto steht. Insgesamt sollen sie drei Minuten lang versuchen, den Kreis immer genau über dem Auto zu halten.“

\subsubsection{Schraubenbrett}

„Sie sehen hier ein Brett mit einigen Gewinden, hier drei Schrauben (zeigen), die es gilt, so schnell wie möglich in diese drei Löcher (zeigen) zu schrauben. Sie brauchen sie nicht ganz fest zu drehen, es reicht, wenn sie leicht anschlagen. Drehen sie nun eine Schraube zur Probe 'rein (schrauben lassen), ... gut, ich lege nun die Schrauben hier nebeneinander (Anordnung neben dem Brett auf der betroffenen Seite, Köpfe nach oben, 2 cm Abstand), es geht los, wenn ich 'Jetzt' sage. .... Jetzt (Stoppuhr drücken, wenn die erste Schraube aufgenommen wird).“

\subsubsection{Erhebung der erwarteten Leistungsteigerung}

„Sie haben.... (Kilogramm bei Kraft, Anschläge bei Schnelligkeit, Watt bei Ausdauer, Fehlerzeit in Prozent bei Koordination I, Sekunden bei Koordination II) erreicht. Wenn Sie nun einzuschätzen versuchen, wieviel sie nach dieser Zeit hier in der Rehabilitation schaffen werden, welchen Wert erwarten sie für sich in dieser Aufgabe?“

\subsubsection{Allgemeine Vorhersage des Wiederherstellungsgrads}

„Wenn Sie im Vergleich zu ihrem früheren Leistungsstand, den wir als $100 \%$ bezeichnen, überlegen, wieviel Leistung sie jetzt insgesamt erbringen, wieviel Prozent wären dies? 
... und auf wieviel Prozent, glauben sie, werden sie nach dieser Rehabilitationsmaßnahme kommen?"“

\subsubsection{Versuchsmaterial, Hilfsmittel und Geräte}

Zusätzlich zu allgemein verfügbaren, versuchsunspezifischen Materialien wie Papier, Bleistift, Stoppuhr und einem Personal Computer (DX 4-86) wurden die im folgenden genauer beschriebenen Materialien verwendet.

\subsubsection{Handdynamometer}

Zur Verwendung kam das 'BASELINE ${ }^{\odot}$ hydraulic hand dynamometer' der Firma 'Smith \& Nephew Rolyan Inc., One Quality Drive, P.O. Box 578, Germantown, WI 53022 U.S.A.', das in Analogie zum 'Jamar dynamometer' der Firma 'Asimow Engineering Co., Los Angeles, CA 90024 U.S.A.' konstruiert wurde. Alle mit dem 'Jamar dynamometer' erhobenen Daten können direkt auf das 'BASELINE ${ }^{\odot}$ hydraulic hand dynamometer' übertragen werden. Es handelt sich hierbei um ein Gerät zur Messung der Griffstärke (bis zu 90 $\mathrm{Kg}$ ) der Hand. Der Handgriff ist zwischen $3.5 \mathrm{~cm}-8.6 \mathrm{~cm}$ auf 5 verschiedene Positionen einstellbar.

\subsubsection{PC-Programmodul ,Tapping“}

Hierbei handelte es sich um ein adaptiertes Word-für-Windows-6.0-Dokument. Bei Aufruf der Datei war in der Mitte ein Balken zu sehen, innerhalb dessen sich am linken Rand eine kleine Figur befand. Diese bewegte sich bei jedem Tastenanschlag der 'Leer-Taste' ein kleines Stück nach rechts (visuelles Feedback, das aber dezent genug war, um nicht abzulenken). Im unteren Bereich des Bildschirms befand sich - für den Patienten im Normalfall nicht als solche identifizierbar - die Zähleinrichtung, die mit jedem Tastenanschlag einen Anschlag mehr anzeigte. Es wurde ein Zeitraum von zehn Sekunden vorgegeben, der von der ersten Tastenberührung an gemessen wurde.

\subsubsection{Fahrradergometer}

Zum Einsatz kam das 'ECB Fitness Ergometer E 420' der Firma 'TUNTURI ${ }^{\odot}$, Tunturipyörä Oy, Fin-20760 Piispanristi'. Da für die Pulsmessung ein externes Gerät eingesetzt wurde, war von den Meßgeräten des Ergometers nur die Leistungsmessung wichtig, ,the left-hand reading in the middle line shows effort in watts (0-995) in steps of five watts“ 
(Tunturipyörä Oy, o. Jahresangabe, S. 3). Das Ergometer hat eine Länge von 108 cm, die Breite beträgt $62 \mathrm{~cm}$ und die Höhe beläuft sich auf $111 \mathrm{~cm}$. Das Gesamtgewicht beträgt 41 $\mathrm{kg}$, von denen $15 \mathrm{~kg}$ auf das Schwungrad entfallen. Die Leistung in Watt als Quotient aus Arbeit und Zeit errechnet sich aus dem Pedalweg $(\mathrm{r}=17.5 \mathrm{~cm})$ und der Kraft pro Zeiteinheit.

\subsubsection{Herzfrequenzmesser}

Verwendet wurde das Modell 'Polar Favor' der Firma 'POLAR ${ }^{\odot}$, Polar Electro GmbH Deutschland, Postfach 1545, D-64505 Groß-Gerau', das nach Herstellerangaben eine EKGgenaue drahtlose Übertragung der Herzfrequenz bietet. Die Ableitung erfolgt über einen Brustgurt mit zwei Elektroden, die Anzeige der Messung erfolgt an einem einer Armbanduhr vergleichbar gestalteten Gerät, das bis zu einer Entfernung von etwa einem Meter die Signale des Brustgurts zuverlässig empfängt.

\subsubsection{PC-Programm RehaCom-Visuomotorik}

Das PC-Programm 'RehaCom' ist eine Programmreihe der Firma HASOMED GmbH, die diese unter der Produktions- und Vertriebsführung von 'Dr. G. Schuhfried Ges.m.b.H., Hyrtlstraße 45, A-2340 Mödlingen' entwickelte. Es handelt sich hierbei um ein computergestütztes Verfahren zur kognitiven Rehabilitation. Das Programmodul 'Visuomotorik' wurde in Anlehnung an bestehende visuomotorische Aufgabenstellungen wie Labyrinthaufgaben, Punktverfolgungstrainings oder Liniennachfahren konzipiert (vgl. Brickenkamp, 1975; Cramon, Mai \& Ziegler, 1995): „Die Indikation ist bei allen Läsionen im motorischen Cortex (Frontallappen) gegeben, im besonderen, wenn Defizite bei der Kontrolle der Feinmotorik bestehen“ (Weber, Regel \& Krause, 1996, S. 19). Da die Tastatur eines Computers bei der Arbeit mit neurologischen Patienten häufig nicht geeignet ist, wurde mit dem RehaCom-Pult „,... ein spezielles Patientenpult entwickelt, das es auch motorisch und kognitiv beeinträchtigten Patienten erlaubt[,] mit RehaCom zu arbeiten“ (a. a. O., S. 3). Ein großer Hebel, im rechten oberen Bereich des Pults angeordnet, dient der Steuerung des 'Hubschraubers' (s. u.). Zu der Aufgabenstellung schreiben die Autoren:

Das Training der Visuomotorischen Koordination erfolgt im vorliegenden Verfahren realitätsnah, indem der Patient als Pilot eines Polizeihubschraubers arbeitet, der über einer Stadt mit Straßen, Häusern, Seen und Bäumen schwebt. Durch die Straßen bewegt sich ein Auto, das mit dem Hubschrauber verfolgt werden muß. Es besteht die 
Aufgabe, den Hubschrauber ständig über dem Fluchtauto „zu führen“. Dabei wird der Hubschrauber durch einen Kreis dargestellt, der mit dem Joystick des RehaComPultes synchron über den Bildschirm bewegt werden kann. .... Verläßt die Mitte des Autos den Hubschrauberkreis, entsteht eine Fehlersituation. Durch visuelles (Blaufärbung des Hubschrauberkreises) und/oder akustisches Feedback (Dauerton) wird auf diese Situation hingewiesen. Sobald sich der Mittelpunkt des Autos wieder im Kreis befindet, wird die Fehlermeldung abgeschaltet. Gemessen und archiviert werden die Fehlerzeit, in der sich das Auto außerhalb des Kreises befand. (Weber, Regel \& Krause, 1995, S. 7; Hervorhebung im Original)

Die Anpassung dieses Trainingsverfahrens zur Koordinationsmessung erfolgte durch die Festlegung der Stufe, der Geschwindigkeit und der Zeit. Die Zeit wurde bei allen Versuchsteilnehmern auf drei Minuten festgeschrieben, während die Schwierigkeitsstufe und die Geschwindigkeit des Fluchtautos individuell variiert wurden, damit durch die sehr unterschiedlichen Koordinationsfähigkeiten der Patienten keine Decken- bzw. Bodeneffekte (vgl. Hager, 1987, S. 240) evoziert wurden. Die variierten Bedingungen wurden notiert und galten bei der zweiten Messung als gegeben. Die Rückmeldung der Fehlerzeit erfolgte in Prozent. Nach den drei Minuten Versuchsdurchlauf sah der Patient auf dem Bildschirm, zu wieviel Zeitanteilen der drei Minuten er sich mit dem Hubschrauberkreis nicht über dem Auto befunden hatte (z. B.: 'In der letzten Aufgabe hatten sie eine Fehlerzeit von 17 \%').

\subsubsection{Schraubenbrett}

Es wurde eine 25 x $25 \mathrm{~cm}$ große Eisenplatte verwendet, in der 33 11-Zoll-Gewinde eingefaßt sind. Diese sind in zwei Siebener-Reihen à drei Schrauben zu einem Kreuz angeordnet, in dessen Mittelbereich (Schnittfläche der 'Balken') sich neun Gewinde befinden (im Aufbau vergleichbar dem Gesellschaftsspiel 'Solitaire'). In der Versuchsreihe waren alle bis auf sechs Schrauben eingedreht. Die sechs freien Gewinde lagen an dem Balkenende, das dem Patienten zugewandt war. In dem Versuch mußten drei Schrauben in die Gewinde gedreht werden, die der Patient unmittelbar vor sich hatte. Die freie Reihe, die für ausreichend Platz beim Schrauben sorgte, lag zwischen der Reihe, die der Patient zu bearbeiten hatte, und dem übrigen Schraubenfeld. 


\subsubsection{Becks Depressions Inventar (BDI)}

„Von depressiven Patienten häufig und von nichtdepressiven Patienten selten berichtete Symptome wurden zu 21, keiner ätiologischen Depressionstheorie verpflichteten Items komprimiert ..." (Hautzinger, Bailer, Worall, Keller \& Beck, 1994, S. 8), so die einleitenden Worte des BDI-Testhandbuchs. Ursprünglich als Fremd- und Selbstbeurteilungsbogen gedacht, setzte es sich jedoch nur als Selbstbeurteilungsbogen durch, dergestalt jedoch, daß es „... zu den am meisten verwendeten Selbstbeurteilungsverfahren zur Messung von Depression“ (Richter, 1991, S. 1) zählt (siehe Anhang B: BDI, S. 221).

Diese einundzwanzig Gruppen bestehen aus jeweils vier einfachen Sätzen, die, in aufsteigender Reihenfolge, zunehmend der ansteigenden Schwere der Störung gerecht werden. Die Patienten kreuzen die Aussage an, die auf sie am meisten zutrifft, bekommen in der Auswertung für die jeweils erste keinen, danach einen bis drei Punkte, die über alle Gruppen aufsummiert werden. Dabei gilt nach Beck, Rush, Shaw und Emery (1994, S. 387) eine Punktzahl unter elf als Indikator für das Nichtvorhandensein einer Depression, bis siebzehn Punkten handelt es sich um eine schwache, bis 26 um eine mäßige und ab 27 Punkten um eine schwere Depression.

Inhaltlich bestehen die 21 Gruppen aus folgenden Dimensionen (Hautzinger, Bailer, Worall \& Keller, 1994, S. 8):

- Traurige Stimmung

- Unzufriedenheit

- Selbsthaß

- Weinen

- Entschlußunfähigkeit

- Schlafstörungen

- Gewichtsverlust
- Pessimismus

- Schuldgefühl

- Selbstanklage

- Reizbarkeit

- Negatives Körperbild

- Ermüdbarkeit

- Hypochondrie
- Versagen

- Strafbedürfnis

- Selbstmordimpulse

- Libidoverlust

- Arbeitsunfähigkeit

- Appetitverlust

- Sozialer Rückzug und Isolierung

Die interne Konsistenz erreichte in der Untersuchung von Kammer (1983) einen Wert von 0.82 (S. 51), in einer anderen Untersuchung, ,the odd-even internal reliability coefficient was 0.86 for this sample, and the BDI total scores correlated 0.65 with clinicians' ratings of depressions“ (Steer, Beck \& Garrison, 1986, S. 124), was konsistent mit den eine Übersicht hinsichtlich noch vielfältiger anderer Untersuchungen zur Güte des BDI abschließenden Worten Hautzingers, Bailers, Worall und Keller (1994) zu sein scheint: „Insgesamt erweist sich das BDI als zuverlässiges, konsistentes, valides, sensibles und 
damit gut brauchbares Instrument zur Messung der Schwere depressiver Symptomatik und deren Veränderung durch Behandlungsmaßnahmen. Die weite Verbreitung des BDI ist angesichts dieser Ergebnisse gerechtfertigt““ (S. 29). Einschränkungen sind nur in bezug auf das Alter zu sehen, einsetzbar ist er zwischen 18 und 80 Jahren (S. 11).

\subsubsection{Rating des Schweregrads der Behinderung}

Um zusätzlich das Ausmaß der Schädigung bzw. der Behinderung zu erheben, wurde die Ratingskala von Boné, Ladurner und Pichler (1988) verwendet. Diese sieht folgende Scores vor (nach Boné, Ladurner \& Pichler, 1988, S. 60):

$0=$ keine Behinderung

1 = eine geringe Behinderung mit minimalen neurologischen Ausfällen, der Patient führt ein unabhängiges Leben

2 = eine mäßige Behinderung mit Vorliegen von neurologischen und/oder intellektuellen Ausfällen

3 = eine schwere Behinderung, wobei der Patient bei Bewußtsein ist, jedoch vollkommen abhängig

$4=$ der Patient ist verstorben

Die Zuordnung der Patienten zu den einzelnen Kategorien erfolgt durch den Leiter der Studie bzw. durch den jeweils verantwortlichen Arzt. Angaben zur Validität und Reliabilität dieser Skala sind in der einschlägigen Literatur noch nicht vorhanden.

\subsection{Versuchsdurchführung}

Die Leitung der Versuche hatten eine Ergo- und eine Physiotherapeutin. Vor Versuchsbeginn nahmen beide Personen an einem supervidierten Training teil, das in Verbindung mit der geringen Versuchsleiterzahl eine hohe Standardisierung in den Versuchsbedingungen gewährleistete. Zur Kontrolle wurden auch während des gesamten Versuchszeitraums in Abständen Probetestungen mit Supervision durchgeführt. In den Versuchen selbst wurden normalerweise nur die standardisierten Instruktionen gegeben, bei einigen Patienten mußte jedoch einige Male zur Erklärung auch ein erweiterter Wortlaut benutzt werden ${ }^{35}$.

\footnotetext{
${ }^{35}$ Im Verlauf der Untersuchung zeigte sich, daß bei der allgemeinen Symptomvielfalt bis hin zu kognitiven Defiziten, die bei neurologischen Patienten auftreten, eine nahezu absolute Standardisierung nicht möglich ist. Dies wird auch in der den Methodenteil einleitenden Erklärung einer Studie aus den USA deutlich: „The 14 subjects of this study were a convenience sample of consecutively available patients who met the following criteria: they were being followed by physical therapy for their first stroke with residual hemipa-
} 
Die Patienten wurden ausnahmslos in Einzelsitzungen getestet, der Versuchsraum war in dieser Zeit geschlossen. Nur die Fahrradergometerbedingung wurde in einem Trainingsraum durchgeführt, in dem einige Male auch andere Patienten anwesend waren.

Die Patienten wurden in der ersten Woche der Rehabilitationsmaßnahme aus dem Aufenthaltsraum zu dem Versuchsraum geleitet. Dort wurden sie informiert, daß zu Beginn und am Ende der Rehabilitation eine Untersuchung durchgeführt wird, die Aufschlüsse über ihre Leistungssteigerung innerhalb der Rehabilitationszeit geben soll. Die Patienten akzeptierten diese Erklärung ausnahmslos und fragten auch während der Versuche nicht weiter nach den Hintergründen der Untersuchung. Der Psychologe, unter dessen Gesamtleitung die Versuche stattfanden, wurde von den Patienten nicht mit den Tests in Verbindung gebracht. Auch eine Beziehung zur Depression wurde von keinem der Patienten hergestellt.

$\mathrm{Zu}$ Beginn wurden persönliche Daten des Patienten auf dem Protokollbogen festgehalten (vgl. Kap. 8.1, S. 220). Hierbei saßen der Patient und die Versuchsleiterin nebeneinander. Die Versuche wurden mit der Hand der geschädigten Seite durchgeführt. Wenn bei tieferen ZNS-Schädigungen (z. B. Hirnstamminsult) beide Seiten betroffen waren, wurde die stärker betroffene Seite ausgewählt. Die Untersuchungsmaterialien befanden sich vor dem Patienten verborgen in einer Kiste, aus der sie zu dem entsprechenden Versuch immer erst herausgenommen wurden.

Als erster Versuchsteil erfolgte mit Hilfe des Trainingsprogramms 'Rehacom' (vgl. Kap. 3.3.2.5, S. 144) die Testung der Feinkoordination der Hand bzw. des Arms ${ }^{36}$ (aus organisatorischen Gründen folgte der Ablauf der Versuchsteile in der Testung nicht der in der Darstellung beibehaltenen Reihenfolge: Kraft, Schnelligkeit, Ausdauer, Koordination I + II). Nach einer Trainings- bzw. Eingewöhnungszeit von $120 \mathrm{sec}$ wurde der eigentliche Versuch begonnen. Nach Abschluß der Aufgabe wurde dem Patienten die Fehlerzeit in Prozent noch einmal gesagt, die er auf dem Bildschirm als objektive Rückmeldung nach Ablauf der drei Minuten vor sich sah, und gefragt, auf welche Prozentzahl er gegen Ende des Rehabilitationsaufenthaltes zu kommen glaube (siehe Versuchsinstruktion, S. 141). Die konkrete Zahl wurde mit dem Ergebnis auf dem Protokollbogen vermerkt. Sichergestellt wurde bei allen

reses, were able to follow three-part verbal instructions, were medically stable, had no other neurologic or orthopedic problems affecting the lower extremities, were able to extend the paretic knee against gravity, had intact sensation of the paretic lower extremity, and provided written informed consent" (Bohannon \& Walsh, 1992, S. 721).

${ }^{36}$ Die Hand durfte nicht aufgelegt werden, so daß eine Kompensation der fehlenden Handkoordination durch die Armkoordination möglich war. 
Aufgaben, daß der Patient die Zusammenhänge von Leistung und Leistungssteigerung verstanden hatte (positiv bei Kraft, Ausdauer, Schnelligkeit, negativ bei beiden Koordinationsaufgaben).

Die Versuchsleiterin wechselte nun das Programm. Der Patient sah ein für die Untersuchung konzipiertes Word-für-Windows-Dokument vor sich, in welchem in der Mitte ein Balken zu sehen war, innerhalb dessen sich ein Punkt befand (siehe Kap. 3.3.2.2, S. 143). Dieser bewegte sich nach rechts, wenn die Leertaste der PC-Tastatur angeschlagen wurde. Der Patient wurde aufgefordert, einige Male die Taste zu betätigen, um ein Gefühl für den Tastendruck und die visuelle Rückmeldung durch den sich bewegenden Punkt zu bekommen. Danach wurde er gebeten, zehn Sekunden lang so häufig wie möglich auf die Taste zu drücken, ohne dabei den Arm oder die Hand aufzulegen. Begonnen wurde die Zeitmessung mit der ersten Berührung. Die Zahl der Kontakte wurde anhand eines eingebauten Zählmechanismus an einer dem Patienten nicht als solche erkennbaren Stelle abgelesen und in den Protokollbogen eingetragen. Insgesamt wurde dieser Versuch drei Male durchgeführt, gewertet wurde die Ausführung mit der höchsten Tappingrate (vgl. Fußnote 38, S. 150). Nun wurde wieder gemäß der Einschätzungsinstruktion die Vorhersage der erwarteten Leistung zum zweiten Testzeitpunkt erhoben und an der entsprechenden Stelle im Protokollbogen vermerkt.

Für den nächsten Versuchsteil wurde der Patient aufgefordert, nach rechts zu rücken, so $\mathrm{da}$ er und die Versuchsleiterin nicht mehr nebeneinander, auf den Bildschirm ausgerichtet, sondern seitlich über Eck saßen. Der Patient bekam das Schraubenbrett vor sich auf den Tisch gestellt (Kap. 3.3.2.6, S. 145), in dem die unteren sechs Löcher, angeordnet in zwei Dreierreihen, frei waren. Drei Schrauben wurden mit je zwei Zentimeter Abstand auf die Seite der geschädigten Hand auf den Tisch gelegt. Um einen Eindruck über Art und Tiefe des Gewindes und die Art der Schrauben zu gewinnen, wurde der Patient aufgefordert, eine Schraube ohne Zeitdruck probehalber bis zu einem leichten Anschlag einzudrehen. Dann wurden die Schrauben wieder richtig angeordnet und es kam die Aufforderung, so schnell wie möglich die drei Schrauben in die unteren drei Löcher einzudrehen; die Zeitmessung wurde mit dem Aufnehmen der ersten Schraube begonnen. Auch hier erfolgte, nachdem die benötigte Zeit eingetragen und dem Patienten genannt wurde, die Erhebung der Vorhersage, die an der entsprechende Stelle vermerkt wurde.

Die Handschlußkraft, die in der Operationalisierung sowohl der Kraft als auch der Belastungswahrnehmung die zentrale Größe darstellte, wurde mit einem Handdynamometer gemessen (Kap. 3.3.2.1, S. 143). Um den Patienten auch hier ein erstes Gespür für das Un- 
tersuchungsgerät zu ermöglichen, wurde ihnen das Gerät in die seitlich nach unten hängende Hand gegeben. Nach einem kleinen Druck nahm die Versuchsleiterin den Dynamometer in die Hand und drehte, ohne daß der Patient eine Rückmeldung über die eigene Kraft bekam, die Marke wieder auf Null zurück. Anschließend drückte sie selbst, während der Patient die Anzeige nun sehen konnte. Durch dieses zweigeteilte Vorgehen sollte erreicht werden, daß der Patient zwar den Zusammenhang von Druck und Zeigerbewegung wahrnehmen konnte, ohne aber den Bezug des eigenen Kraftschlusses zu einer objektivierbaren Zahl zu haben ${ }^{37}$. Nun wurde gemäß der Instruktion (Kap. 3.3.1, S. 141) erst die hypostasierte Hälfte der Maximalkraft erhoben, darauf die Maximalkraft und die Vorhersage des erwarteten Leistungszuwachses. Zur Ermittlung der Maximalkraft wurden ebenfalls in kurzen Abständen drei Versuche durchgeführt, von denen der beste in die Wertung einging. Die Werte wurden unmittelbar nach der Erhebung vermerkt. Ziel dieses Vorgehens war es, vergleichbar dem Vorgehen beim Tapping (s. o.), einen Wert möglichst nahe dem Maximalwert, anstatt eines mittleren Leistungskoeffizienten, zu erheben ${ }^{38}$.

Da in Anamnesegesprächen im Rahmen der neurologischen Rehabilitation Patienten häufig mit Prozentzahlen operieren, um das Ausmaß des Leistungseinbruchs durch die Krankheit bzw. der erwarteten Besserung zu formulieren, wurde zusätzlich zu den Tests auch eine allgemeine Einschätzung erhoben. Hierbei galt es festzustellen, welchen Prozentwert der allgemeinen Leistungsfähigkeit sich der Patient zum Zeitpunkt der Untersuchung geben würde und auf welchen er zu kommen erwarte. Als Referenzwert wurde das prämorbide Leistungsniveau mit $100 \%$ festgesetzt. Diese Werte wurden aufgeschrieben. Der Patient erhielt ein BDI (siehe Kap. 3.3.2.6, S. 145) mit der Bitte, dieses noch am gleichen Tag auszufüllen. Auch hier gaben sich die Patienten mit der Versicherung zufrieden, daß der Bogen zu der Untersuchung gehöre, die Daten anonym behandelt und keiner anderen offiziellen Stelle (Krankenkasse) zugänglich gemacht würden.

\footnotetext{
${ }^{37}$ Damit konnte einerseits eine Konfundierung mit der anschließenden Erhebung der halben Maximalkraft ausgeschlossen werden. Andererseits war das initiale Drücken an dieser Stelle besonders wichtig, da mit zunehmendem Druck bei dem Handdynamometer kein spürbarer Weg zurückgelegt wird, die Versuchsperson daher den Eindruck hätte haben können, daß sich ,,ja gar nichts tut“. Dies hätte in der Aufgabe, nur mit der halben Kraft zu drücken, zu einer starken Verzerrung führen können, da in der ersten Überraschung wahrscheinlich stärker gedrückt worden wäre, um einen subjektiv spürbaren Effekt zu haben.

${ }^{38}$ In einem Test, in dem die beobachtete Leistung um die tatsächliche variiert, geht die Klassische Testtheorie von einer Normalverteilung der beobachteten Werte über den tatsächlich zugrundeliegenden aus (Fisseni, 1990). In diesem Fall wäre es sinnvoll, die mittlere Leistung als die eigentliche anzusehen. In dem vorliegenden Fall kann aber die beobachtbare Leistung die tatsächliche per definitionem nicht überschreiten (maximale Kraft bzw. maximale Geschwindigkeit), so daß mit der besten Versuchsdurchführung die der tatsächlichen Leistung näherstehende gewählt wurde.
} 
Anschließend wurde dem Patienten der Brustgurt für die Messung der Herzfrequenz angelegt und ging mit der Versuchsleiterin in den Nachbarraum, in dem das Fahrradergometer stand. Dieses wurde für die jeweilige Person richtig eingestellt. Nun wurde der Patient gebeten, sich auf einer leichten Stufe vier Minuten einzufahren ${ }^{39}$. Alle zwei Minuten erfolgte eine Leistungssteigerung, bis eine Herzfrequenz von 130 Schlägen/min erreicht wurde (PWC 13040). Der Patient wurde gefragt, bei wieviel Watt er diese Herzfrequenz in der Abschlußuntersuchung zu erreichen glaube. Die Werte wurden notiert. Abschließend dankte die Versuchsleiterin dem Patienten und geleitete ihn wieder in den Aufenthaltsraum. Die Dauer dieser Versuche betrug etwa 45 min.

Es wurde darauf geachtet, daß die Versuche am Ende des Rehabilitationsaufenthaltes $\left(\mathrm{t}_{2}\right)$ - abgesehen von der Erhebung der Leistungsvorhersagen und der Ausgabe des Depressionsinventars - genau dem gleichen Ablauf folgten. Der Zeitpunkt der zweiten Messung lag in der Regel in der vierten bis sechsten Woche des Rehabilitationsaufenthaltes des Patienten.

\footnotetext{
${ }^{39}$ In der Regel wurde ein Wert von vier Minuten angestrebt. Doch auch hier zeigte sich, daß der Alltag neurologischer Patienten und ein standardisiertes Vorgehen nur bedingt vereinbar sind (vgl. Fußnote 35, S. 147). So mußte manchmal auf die volle Einfahrzeit verzichtet werden.

${ }^{40}$ In Einzelfällen mußte die Adaptation auf einem noch geringeren Niveau erfolgen. Bei Patienten mit BetaBlocker-Medikation oder mit sehr reduzierter Leistungsfähigkeit wurde auch mit einem PWC von 90 - 110 gearbeitet, der aber für den intraindividuellen Leistungsvergleich dann auch zum zweiten Zeitpunkt herangezogen wurde.
} 


\section{Ergebnis}

Die folgenden Abbildungen zeigen die Verteilung der Werte (X-Achse: Kriteriumsvariable; Y-Achse: Prädiktorvariable; vgl. S. 134) der statistischen Hypothesen. Da durch den Ausfall von Einzelwerten (vgl. Fußnote 35, S. 140; siehe auch Anhang D-J, S. 223 ff.) die Anzahl der Versuchspersonen pro Bedingung schwankte, wird im folgenden für jeden Versuchteil auch die Anzahl der Versuchspersonen (n) angegeben. Anschließend wird jeweils die Signifikanzprüfung vorgenommen. Hieraus ergibt sich die Beibehaltung bzw. Annahme oder die Verwerfung bzw. Ablehnung der statistischen Null- und Alternativhypothesen.

\subsection{Darstellung der $\mathbf{H} 1$}

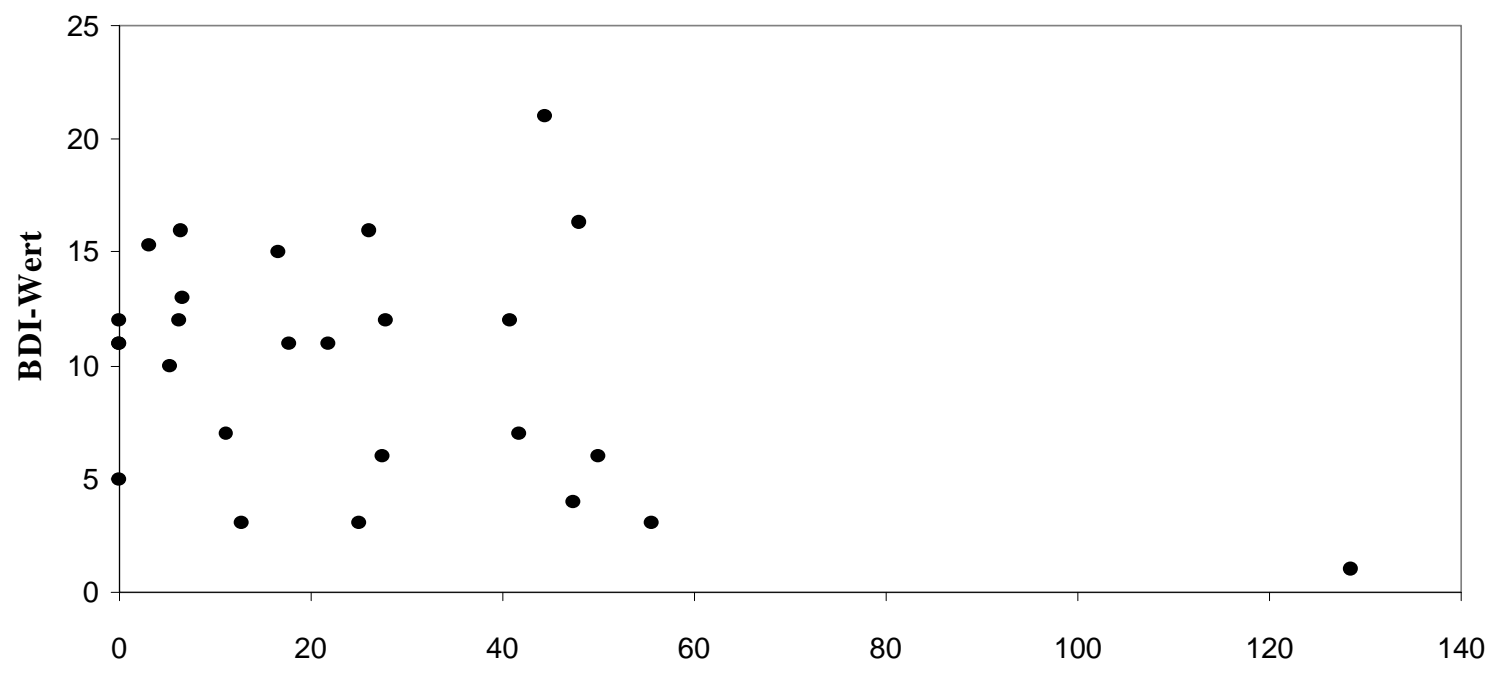

Abweichung der Vorhersage von der tatsächlichen Leistungssteigerung in Prozent

Abbildung 5: Genauigkeit der Vorhersage des Kraftzuwachses in Abhängigkeit vom Grad der Depressivität

Die Korrelation zwischen dem Grad der Depressivität und der Genauigkeit der Vorhersage beträgt bei $n=26$

$$
\mathbf{r}=-.36
$$


Die statistische Signifikanzprüfung erfolgt gemäß Bortz (1989, S. 261) mit Hilfe des tTests:

$$
\mathrm{t}=\frac{\mathrm{r} \cdot \sqrt{\mathrm{n}-2}}{\sqrt{1-\mathrm{r}^{2}}}=\frac{-0.3557 \cdot \sqrt{26-2}}{\sqrt{1-0.3557^{2}}}=-1.8645
$$

Der t-Wert, der für eine Signifikanz auf dem $5 \%$-Niveau $(\alpha=.05)$ erreicht werden müßte, liegt bei $\mathrm{t}_{(24, \text { einseitig) }}=-1.711$ (Bortz, 1989, S. 840). Somit ist der Zusammenhang zwischen dem Depressionsgrad und der Genauigkeit der Einschätzung signifikant.

Die $\mathrm{H}_{0,1-\text { Kraft }}$ wird verworfen ${ }^{41}$, die $\mathrm{H}_{1,1-\text { Kraft }}$ kann angenommen werden.

${ }^{41}$ „Die Nullhypothese stellt in der klassischen Prüfstatistik die Basis dar, von der aus entschieden wird, ob die Alternativhypothese akzeptiert werden kann oder nicht. Nur wenn die Realität 'praktisch' nicht mit der Nullhypothese zu erklären ist, darf sie zugunsten der neuen Alternativhypohthese verworfen werden“ (Bortz, 1987, S. 144; kursiv im Original). Somit kann die Nullhypothese beibehalten oder verworfen, die Alternativhypothese (H1) angenommen oder abgelehnt werden. 


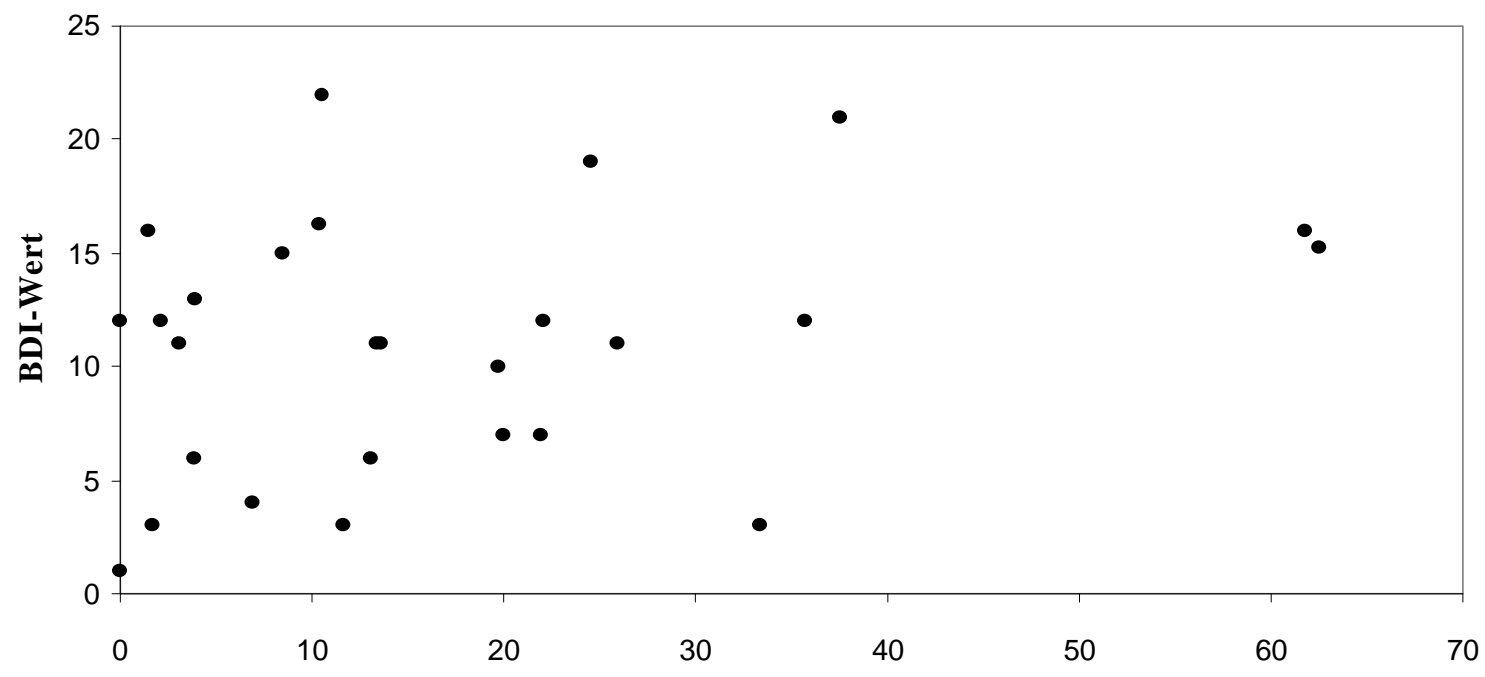

Abweichung der Vorhersage von der tatsächlichen Leistungssteigerung in Prozent

Abbildung 6: Genauigkeit der Vorhersage des Schnelligkeitszuwachses in Abhängigkeit vom Grad der Depressivität

Die Korrelation zwischen dem Grad der Depressivität und der Genauigkeit der Vorhersage beträgt bei $n=27$

$$
\mathbf{r}=\mathbf{. 3 0}
$$

Die statistische Signifikanzprüfung erfolgt gemäß Bortz (1989, S. 261) mit Hilfe des tTests:

$$
\mathrm{t}=\frac{\mathrm{r} \cdot \sqrt{\mathrm{n}-2}}{\sqrt{1-\mathrm{r}^{2}}}=\frac{0.3049 \cdot \sqrt{27-2}}{\sqrt{1-0.3049^{2}}}=1.6007
$$

Der t-Wert, der für eine Signifikanz auf dem $5 \%$-Niveau $(\alpha=.05)$ erreicht werden

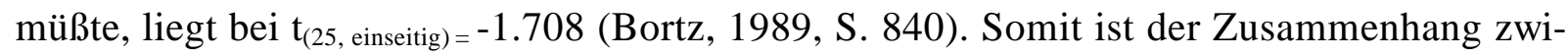
schen dem Depressionsgrad und der Genauigkeit der Einschätzung nicht signifikant.

Die $\mathrm{H}_{0,1-\text { Schnelligkeit }}$ wird beibehalten, die $\mathrm{H}_{1,1-\text { Schnelligkeit }}$ muß abgelehnt werden. 


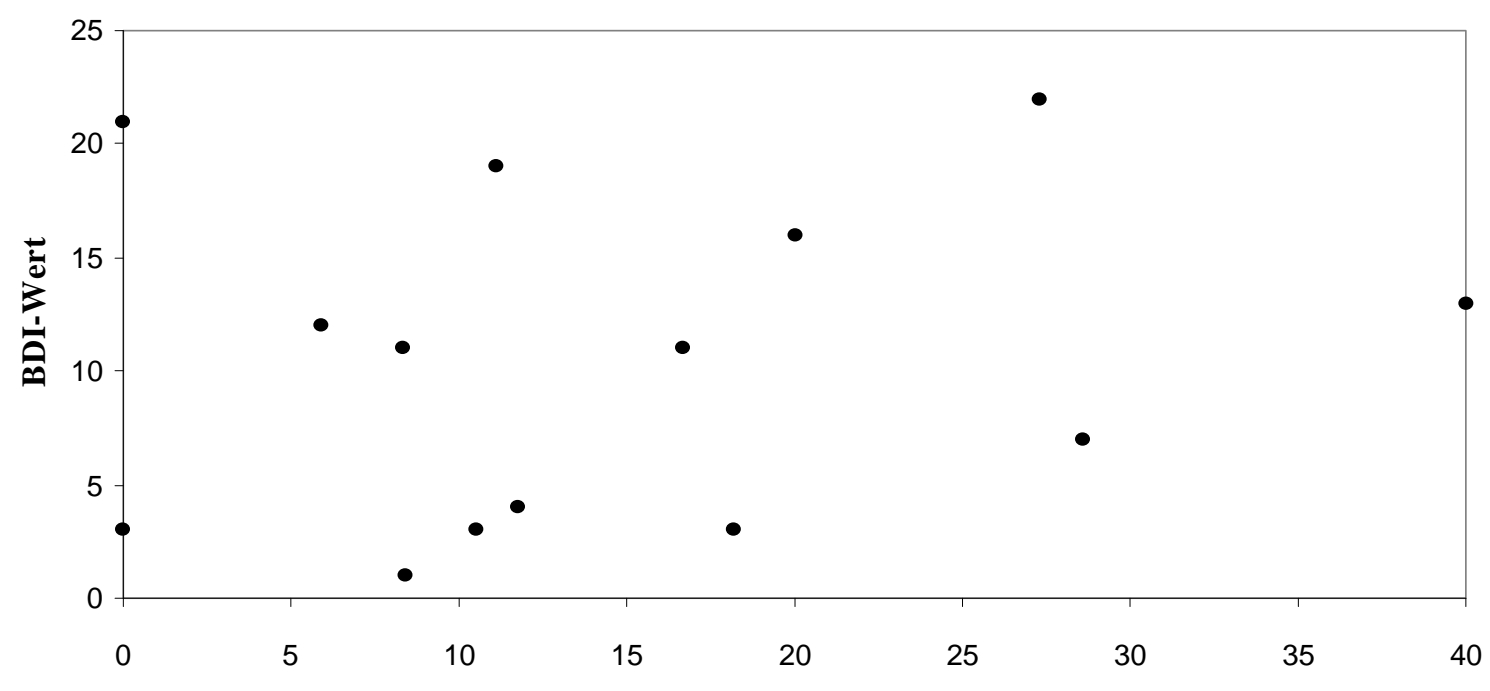

Abweichung der Vorhersage von der tatsächlichen Leistungssteigerung in Prozent

Abbildung 7: Genauigkeit der Vorhersage des Ausdauerzuwachses in Abhängigkeit vom Grad der Depressivität

Die Korrelation zwischen dem Grad der Depressivität und der Genauigkeit der Vorhersage beträgt bei $n=14$

$$
\mathbf{r}=.17
$$

Die statistische Signifikanzprüfung erfolgt gemäß Bortz (1989, S. 261) mit Hilfe des tTests:

$$
\mathrm{t}=\frac{\mathrm{r} \cdot \sqrt{\mathrm{n}-2}}{\sqrt{1-\mathrm{r}^{2}}}=\frac{0.1731 \cdot \sqrt{14-2}}{\sqrt{1-0.1731^{2}}}=0.6088
$$

Der t-Wert, der für eine Signifikanz auf dem $5 \%$-Niveau $(\alpha=.05)$ erreicht werden

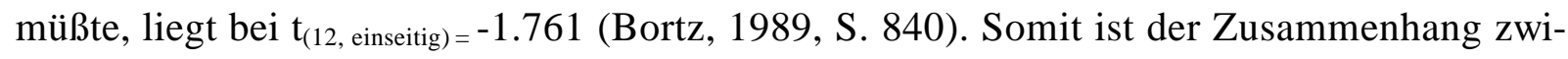
schen dem Depressionsgrad und der Genauigkeit der Einschätzung nicht signifikant.

Die $\mathrm{H}_{0,1 \text {-Ausdauer }}$ wird beibehalten, die $\mathrm{H}_{1,1 \text {-Ausdauer }}$ muß abgelehnt werden. 


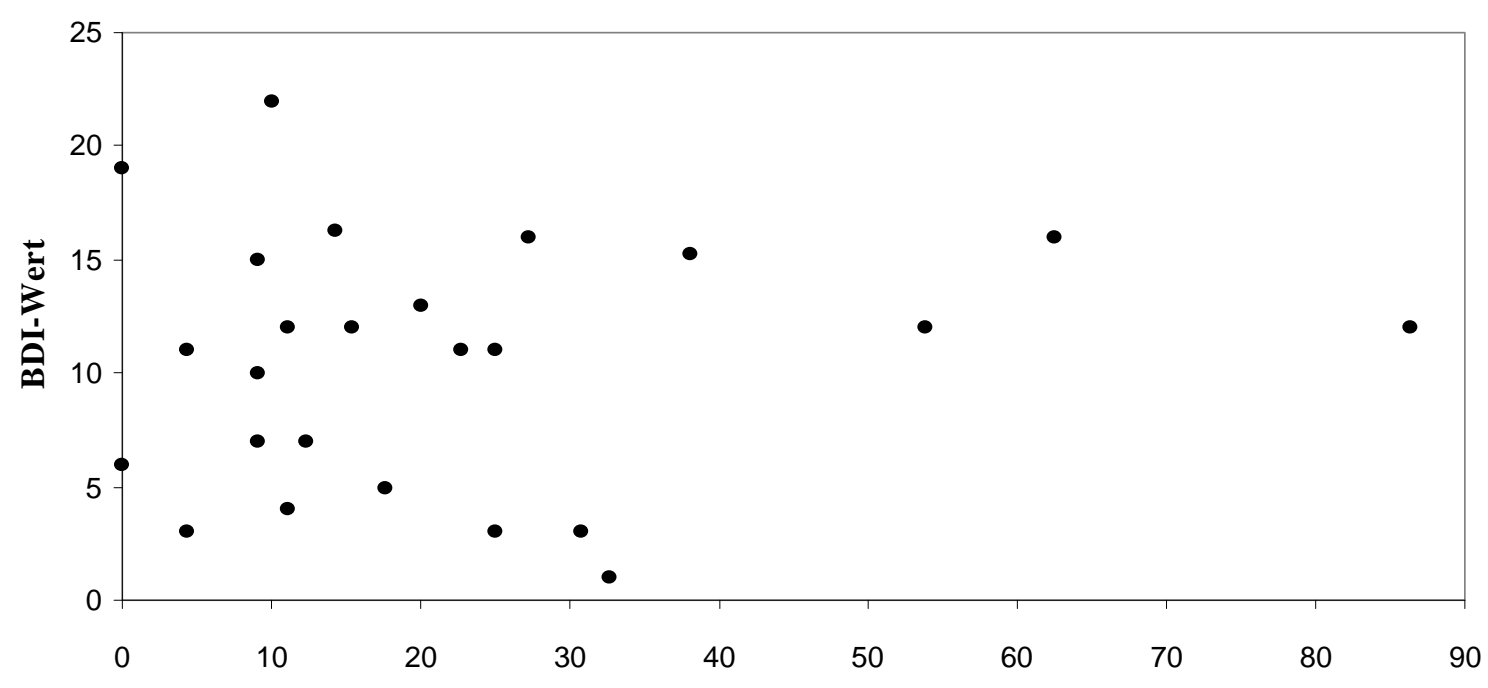

Abweichung der Vorhersage von der tatsächlichen Leistungssteigerung in Prozent

Abbildung 8: Genauigkeit der Vorhersage des Koordinations-I-Zuwachses in Abhängigkeit vom Grad der Depressivität

Die Korrelation zwischen dem Grad der Depressivität und der Genauigkeit der Vorhersage beträgt bei $n=25$

$$
\mathbf{r}=.08
$$

Die statistische Signifikanzprüfung erfolgt gemäß Bortz (1989, S. 261) mit Hilfe des tTests:

$$
\mathrm{t}=\frac{\mathrm{r} \cdot \sqrt{\mathrm{n}-2}}{\sqrt{1-\mathrm{r}^{2}}}=\frac{0.0846 \cdot \sqrt{25-2}}{\sqrt{1-0.0846^{2}}}=0.4072
$$

Der t-Wert, der für eine Signifikanz auf dem $5 \%$-Niveau $(\alpha=.05)$ erreicht werden

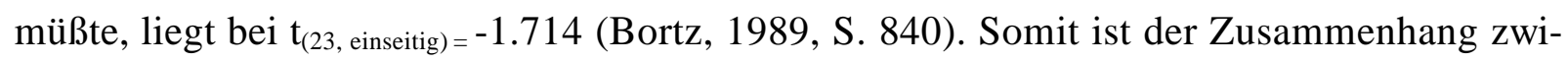
schen dem Depressionsgrad und der Genauigkeit der Einschätzung nicht signifikant.

Die $\mathrm{H}_{0,1 \text {-Koordination I }}$ wird beibehalten, die $\mathrm{H}_{1,1 \text {-Koordination I }} \mathrm{muß}$ abgelehnt werden. 


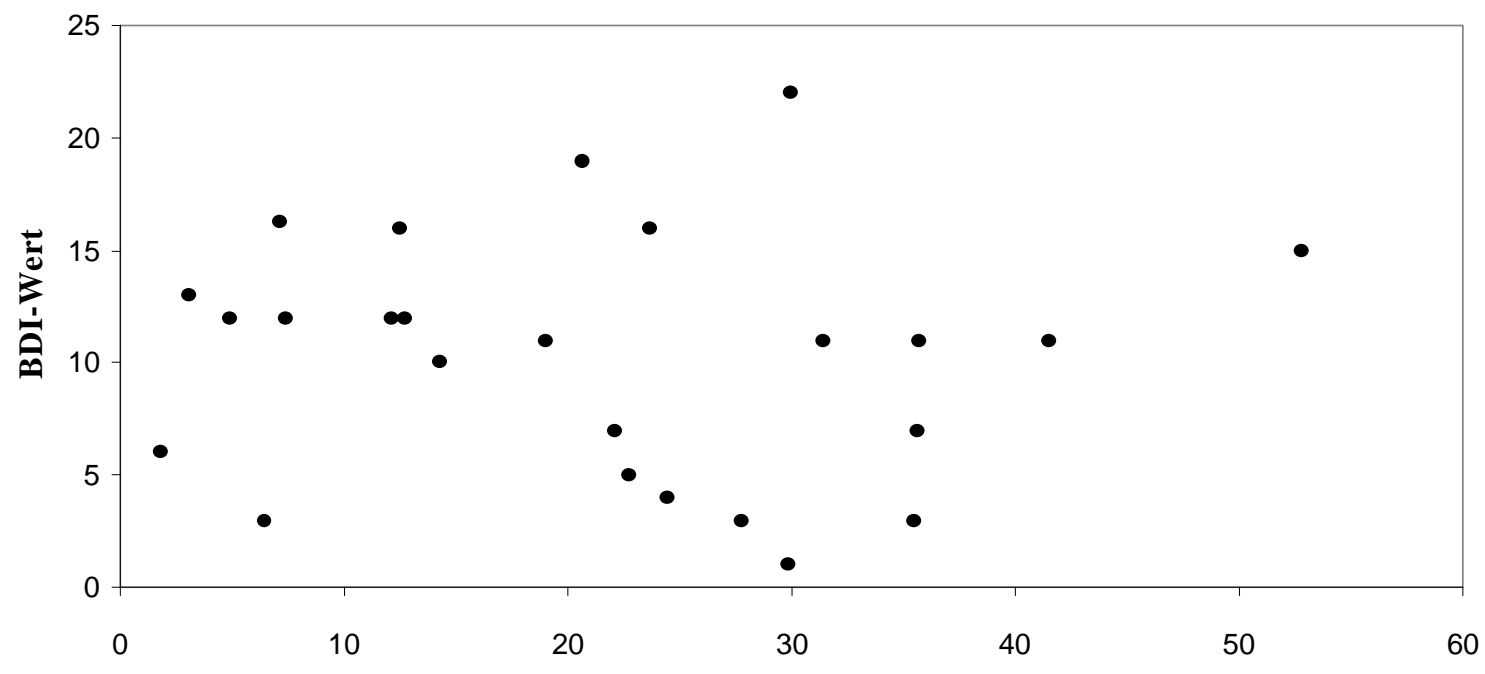

Abweichung der Vorhersage von der tatsächlichen Leistungssteigerung in Prozent

Abbildung 9: Genauigkeit der Vorhersage des Koordination-II-Zuwachses in Abhängigkeit vom Grad der Depressivität

Die Korrelation zwischen dem Grad der Depressivität und der Genauigkeit der Vorhersage beträgt bei $n=25$

$$
\mathbf{r}=\mathbf{- . 0 5}
$$

Die statistische Signifikanzprüfung erfolgt gemäß Bortz (1989, S. 261) mit Hilfe des tTests:

$$
\mathrm{t}=\frac{\mathrm{r} \cdot \sqrt{\mathrm{n}-2}}{\sqrt{1-\mathrm{r}^{2}}}=\frac{-0.0533 \cdot \sqrt{25-2}}{\sqrt{1-0.0533^{2}}}=-0.2560
$$

Der t-Wert, der für eine Signifikanz auf dem $5 \%$-Niveau $(\alpha=.05)$ erreicht werden

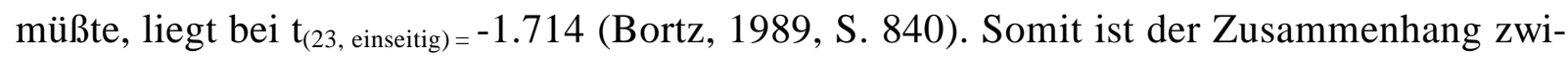
schen dem Depressionsgrad und der Genauigkeit der Einschätzung nicht signifikant.

Die $\mathrm{H}_{0,1-\text { Koordination II }}$ wird beibehalten, die $\mathrm{H}_{1,1 \text {-Koordination II }}$ muß abgelehnt werden. 


\subsection{Darstellung der $\mathbf{H} 2$}

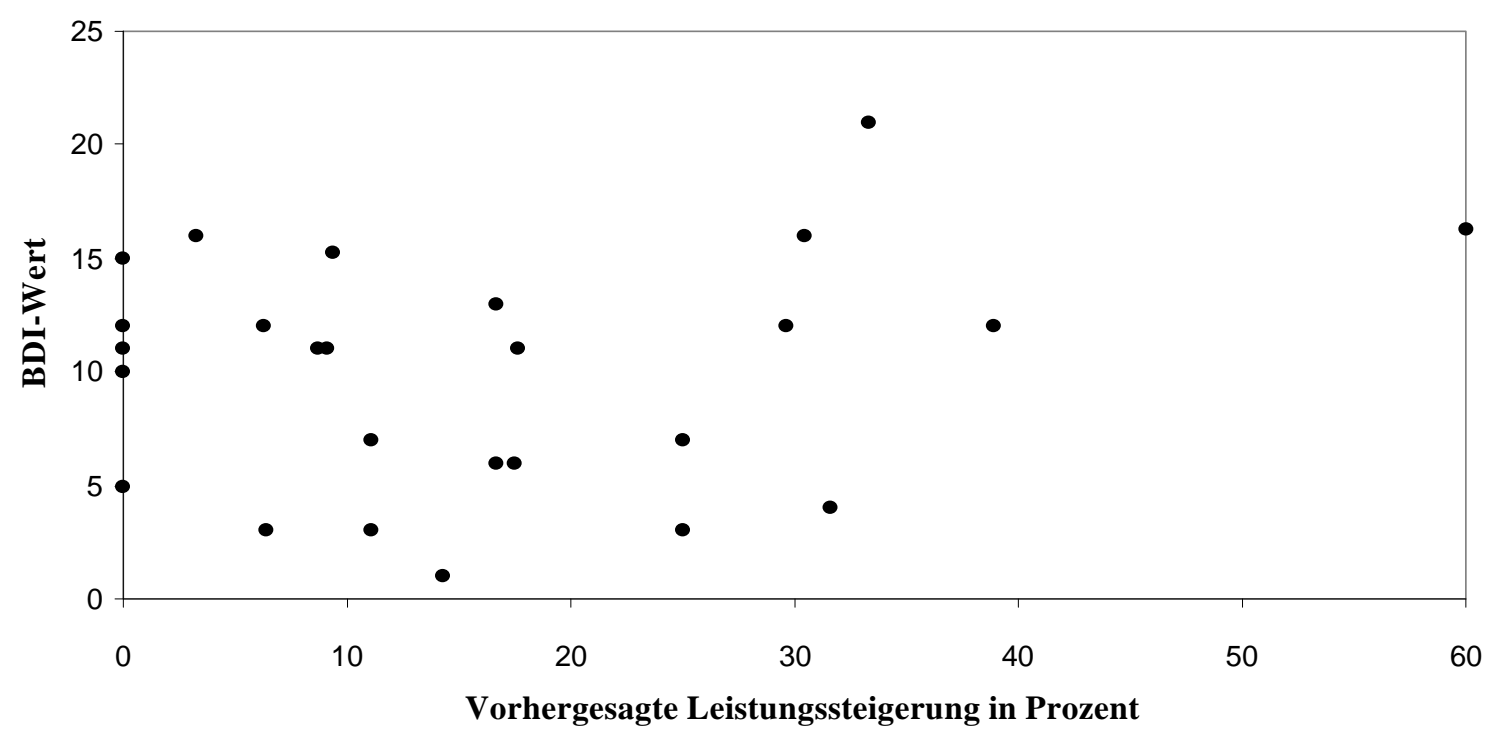

Abbildung 10: Vorhersage des Kraftzuwachses in Abhängigkeit vom Grad der Depressivität

Die Korrelation zwischen dem Grad der Depression und der Vorhersage beträgt bei $\mathrm{n}=26$

$$
\mathbf{r}=\mathbf{- . 0 2} \text {. }
$$

Die statistische Signifikanzprüfung erfolgt gemäß Bortz (1989, S. 261) mit Hilfe des tTests:

$$
\mathrm{t}=\frac{\mathrm{r} \cdot \sqrt{\mathrm{n}-2}}{\sqrt{1-\mathrm{r}^{2}}}=\frac{-0.0152 \cdot \sqrt{26-2}}{\sqrt{1-0.0152^{2}}}=-0.0745
$$

Der t-Wert, der für eine Signifikanz auf dem $5 \%$-Niveau $(\alpha=.05)$ erreicht werden

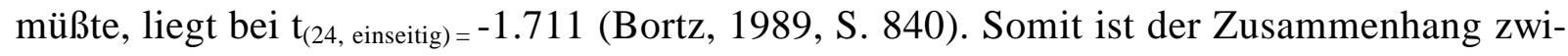
schen dem Depressionsgrad und der Vorhersage nicht signifikant.

Die $\mathrm{H}_{0,2-\text { Kraft }}$ wird beibehalten, die $\mathrm{H}_{1,2 \text {-Kraft }}$ muß abgelehnt werden. 


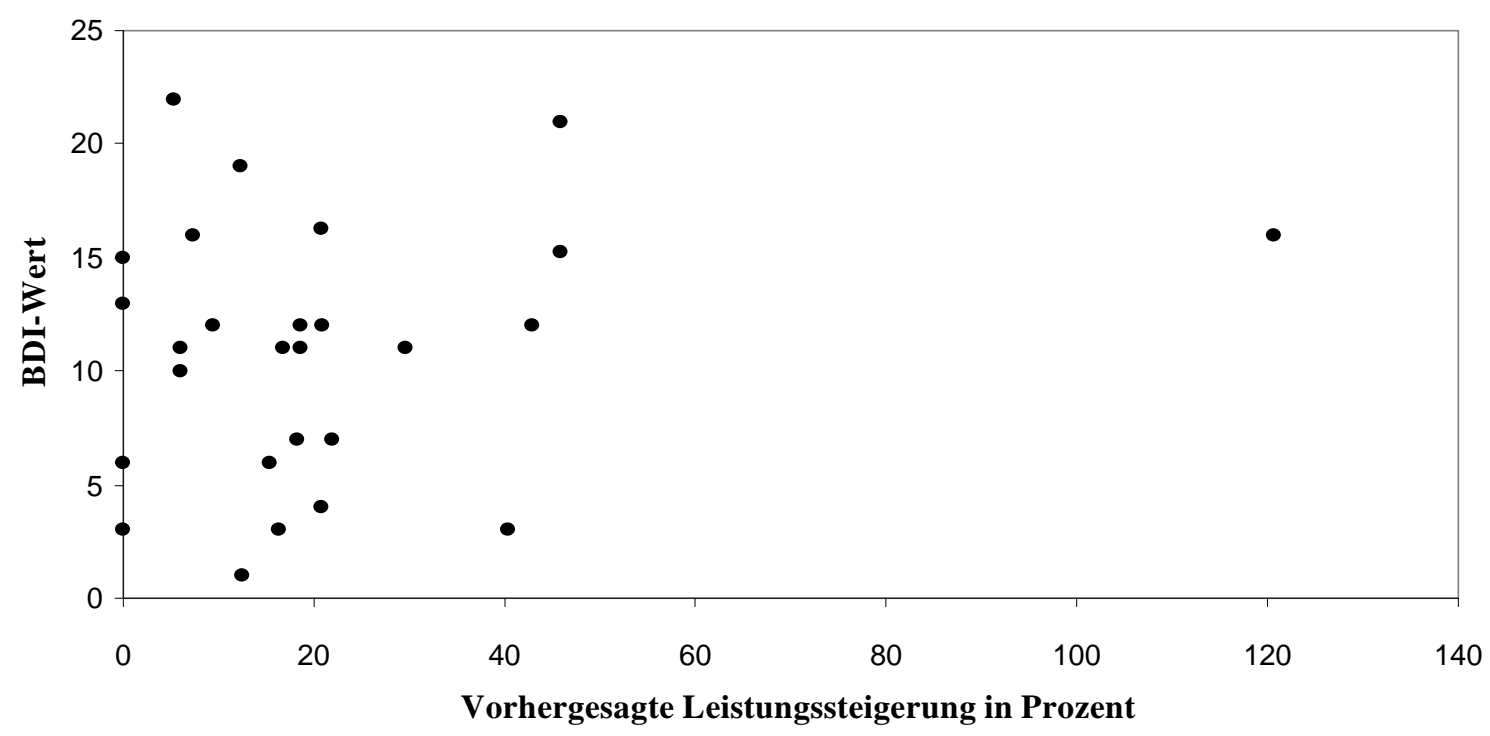

Abbildung 11: Vorhersage des Schnelligkeitszuwachses in Abhängigkeit vom Grad der Depressivität

Die Korrelation zwischen dem Grad der Depression und der Vorhersage beträgt bei $\mathrm{n}=27$

$$
\mathbf{r}=.20
$$

Die statistische Signifikanzprüfung erfolgt gemäß Bortz (1989, S. 261) mit Hilfe des tTests:

$$
\mathrm{t}=\frac{\mathrm{r} \cdot \sqrt{\mathrm{n}-2}}{\sqrt{1-\mathrm{r}^{2}}}=\frac{0.2038 \cdot \sqrt{27-2}}{\sqrt{1-0.2038^{2}}}=1.0408
$$

Der t-Wert, der für eine Signifikanz auf dem $5 \%$-Niveau $(\alpha=.05)$ erreicht werden

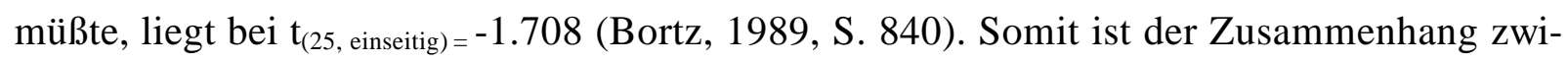
schen dem Depressionsgrad und der Vorhersage nicht signifikant.

Die $\mathrm{H}_{0,2 \text {-Schnelligkeit }}$ wird beibehalten, die $\mathrm{H}_{1,2 \text {-Schnelligkeit }}$ muß abgelehnt werden. 


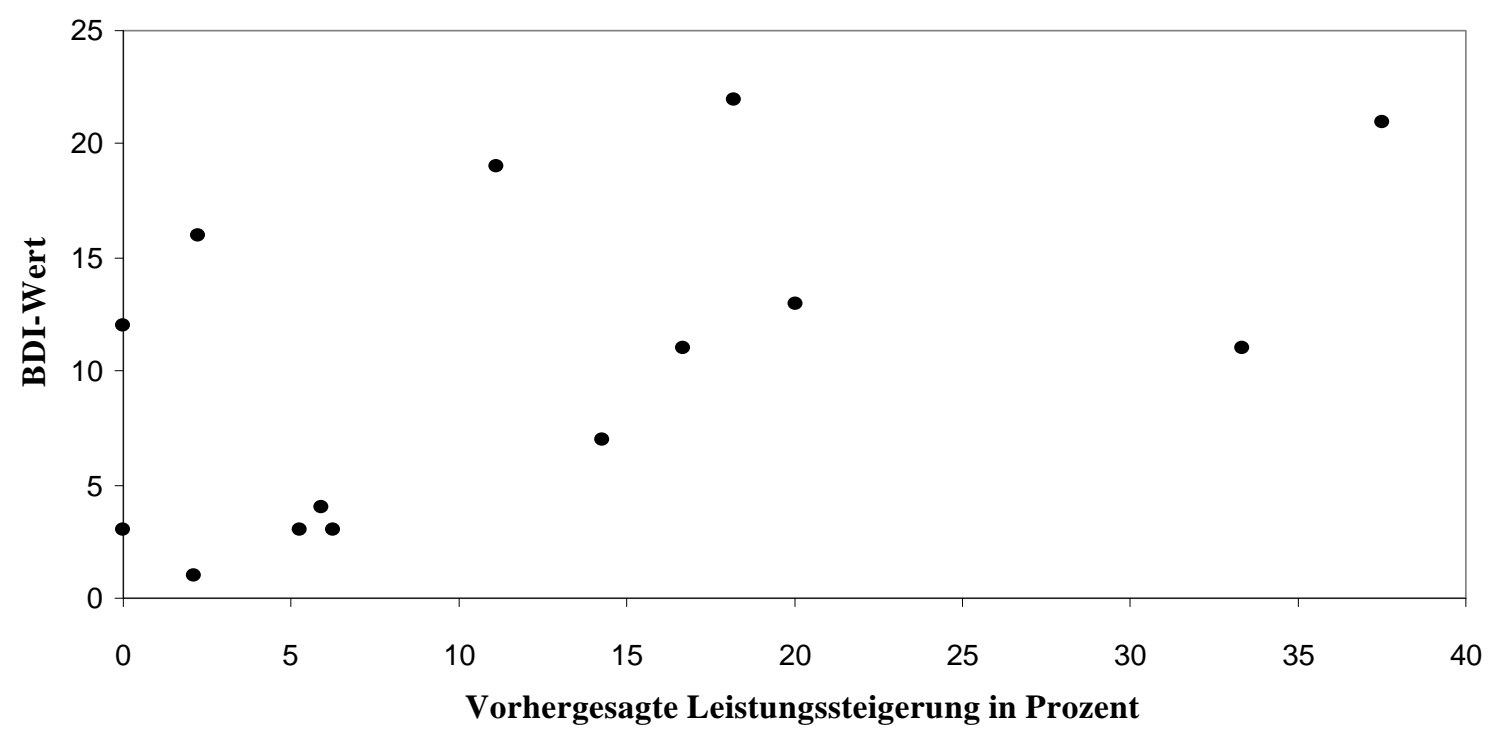

Abbildung 12: Vorhersage des Ausdauerzuwachses in Abhängigkeit vom Grad der Depressivität

Die Korrelation zwischen dem Grad der Depression und der Vorhersage beträgt bei $\mathrm{n}=14$

$$
\mathbf{r}=.54
$$

Die statistische Signifikanzprüfung erfolgt gemäß Bortz (1989, S. 261) mit Hilfe des tTests:

$$
\mathrm{t}=\frac{\mathrm{r} \cdot \sqrt{\mathrm{n}-2}}{\sqrt{1-\mathrm{r}^{2}}}=\frac{0.5448 \cdot \sqrt{14-2}}{\sqrt{1-0.5448^{2}}}=2.2506
$$

Der t-Wert, der für eine Signifikanz auf dem $5 \%$-Niveau $(\alpha=.05)$ erreicht werden

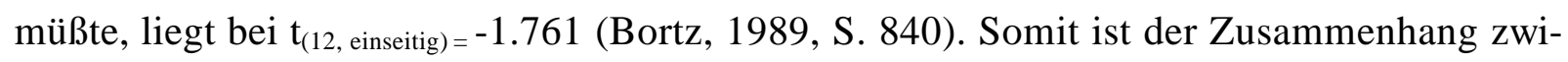
schen dem Depressionsgrad und der Vorhersage nicht signifikant.

Die $\mathrm{H}_{0,2 \text {-Ausdauer }}$ wird beibehalten, die $\mathrm{H}_{1,2 \text {-Ausdauer }}$ muß abgelehnt werden. 


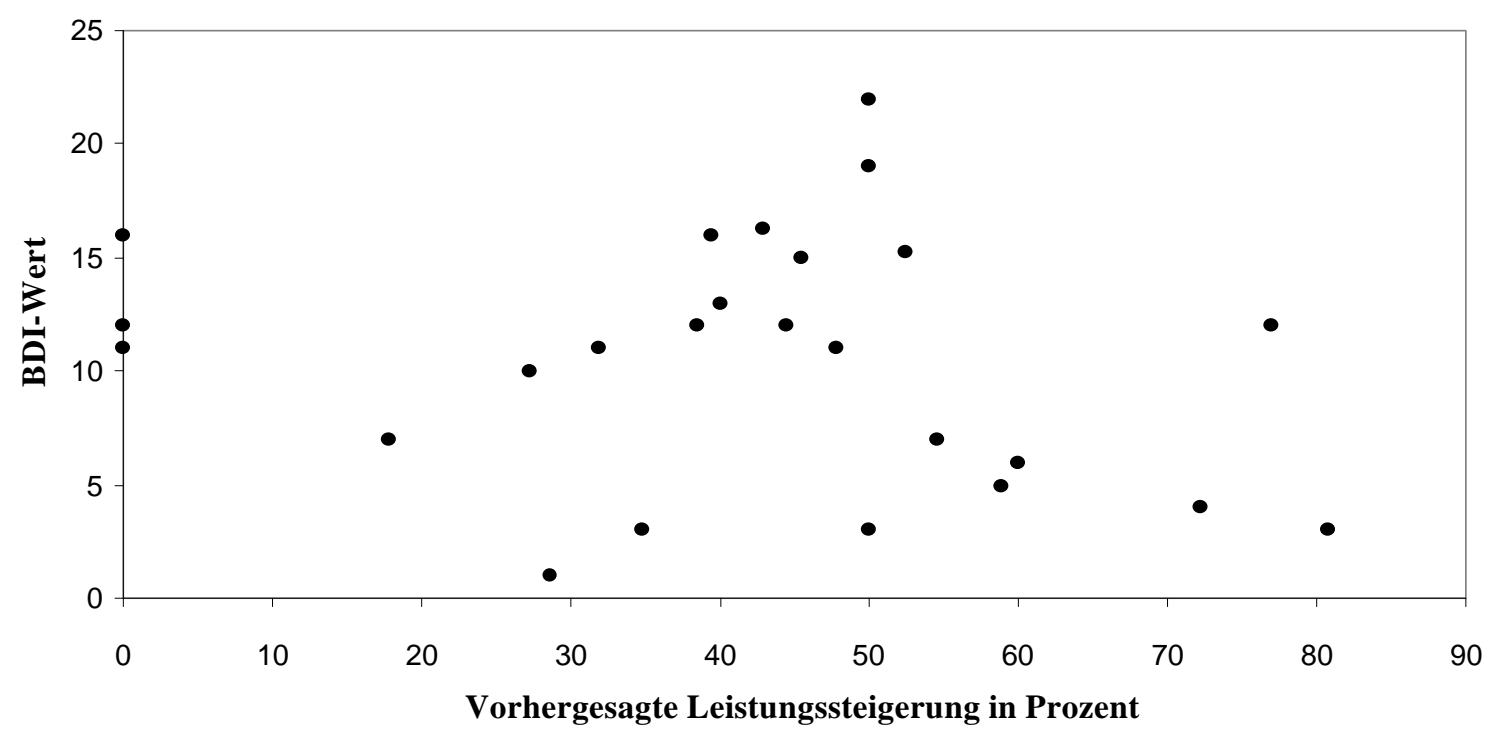

Abbildung 13: Vorhersage des Koordination-I-Zuwachses in Abhängigkeit vom Grad der Depressivität

Die Korrelation zwischen dem Grad der Depression und der Vorhersage beträgt bei $\mathrm{n}=25$

$$
\mathbf{r}=-\mathbf{2 0}
$$

Die statistische Signifikanzprüfung erfolgt gemäß Bortz (1989, S. 261) mit Hilfe des tTests:

$$
\mathrm{t}=\frac{\mathrm{r} \cdot \sqrt{\mathrm{n}-2}}{\sqrt{1-\mathrm{r}^{2}}}=\frac{-0.1989 \cdot \sqrt{25-2}}{\sqrt{1-0.1989^{2}}}=-0.9733
$$

Der t-Wert, der für eine Signifikanz auf dem $5 \%$-Niveau $(\alpha=.05)$ erreicht werden müßte, liegt bei $t_{(23, \text { einseitig) }}=-1.714$ (Bortz, 1989, S. 840). Somit ist der Zusammenhang zwischen dem Depressionsgrad und der Vorhersage nicht signifikant.

Die $\mathrm{H}_{0,2-\text { Koordination I }}$ wird beibehalten, die $\mathrm{H}_{1,2 \text {-Koordination I }} \mathrm{muß}$ abgelehnt werden. 


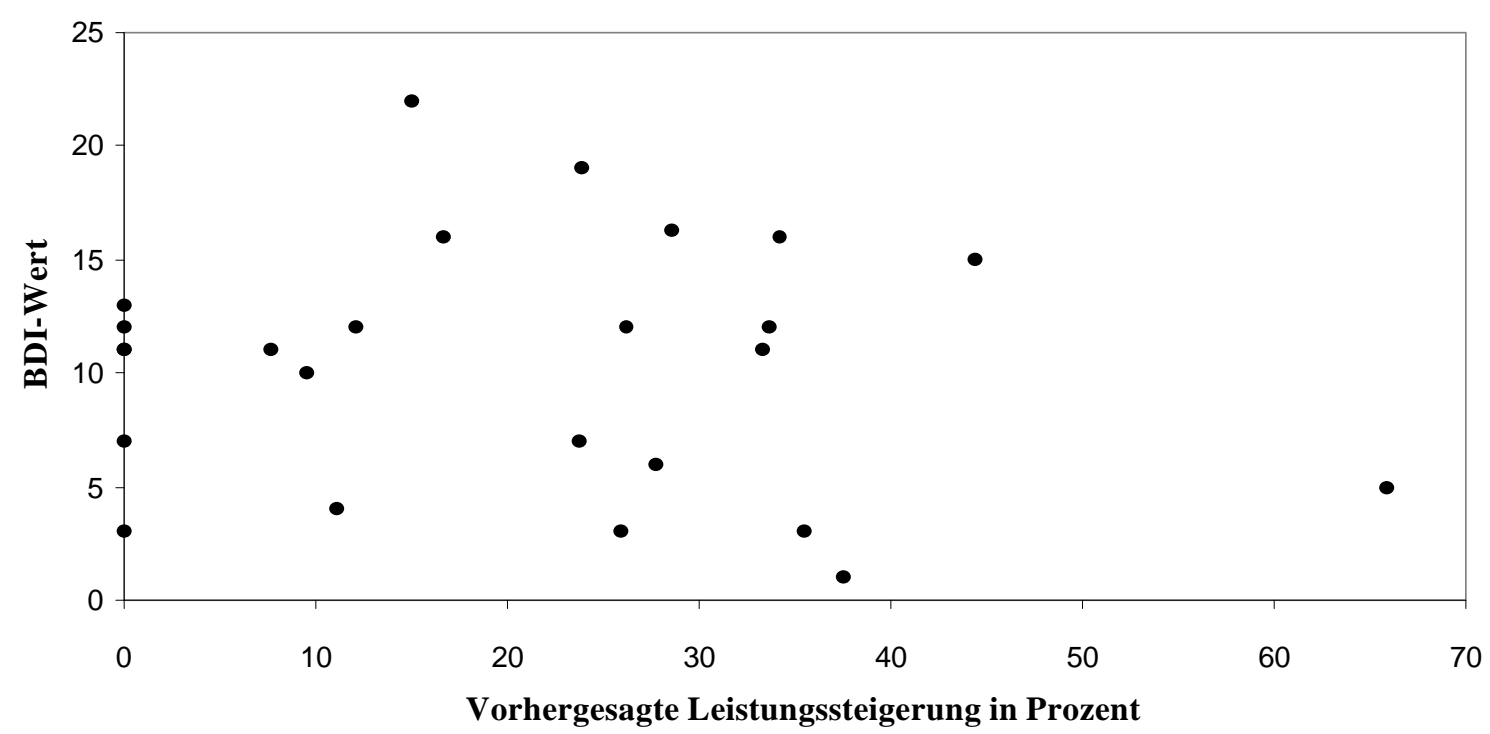

Abbildung 14: Vorhersage des Koordination-II-Zuwachses in Abhängigkeit vom Grad der Depressivität

Die Korrelation zwischen dem Grad der Depression und der Vorhersage beträgt bei $\mathrm{n}=25$

$$
\mathbf{r}=-.14
$$

Die statistische Signifikanzprüfung erfolgt gemäß Bortz (1989, S. 261) mit Hilfe des tTests:

$$
\mathrm{t}=\frac{\mathrm{r} \cdot \sqrt{\mathrm{n}-2}}{\sqrt{1-\mathrm{r}^{2}}}=\frac{-0.1397 \cdot \sqrt{25-2}}{\sqrt{1-0.1397^{2}}}=-0.6766
$$

Der t-Wert, der für eine Signifikanz auf dem $5 \%$-Niveau $(\alpha=.05)$ erreicht werden

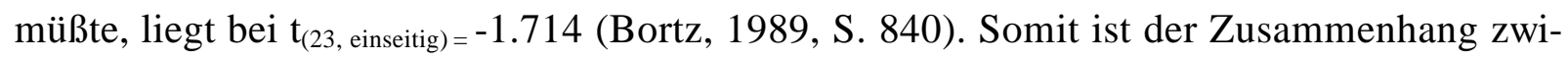
schen dem Depressionsgrad und der Vorhersage nicht signifikant.

Die $\mathrm{H}_{0,2 \text {-Koordination II }}$ wird beibehalten, die $\mathrm{H}_{1,2 \text {-Koordination II }} \mathrm{mu}$ abgelehnt werden. 


\subsection{Darstellung der $\mathbf{H} 3$}

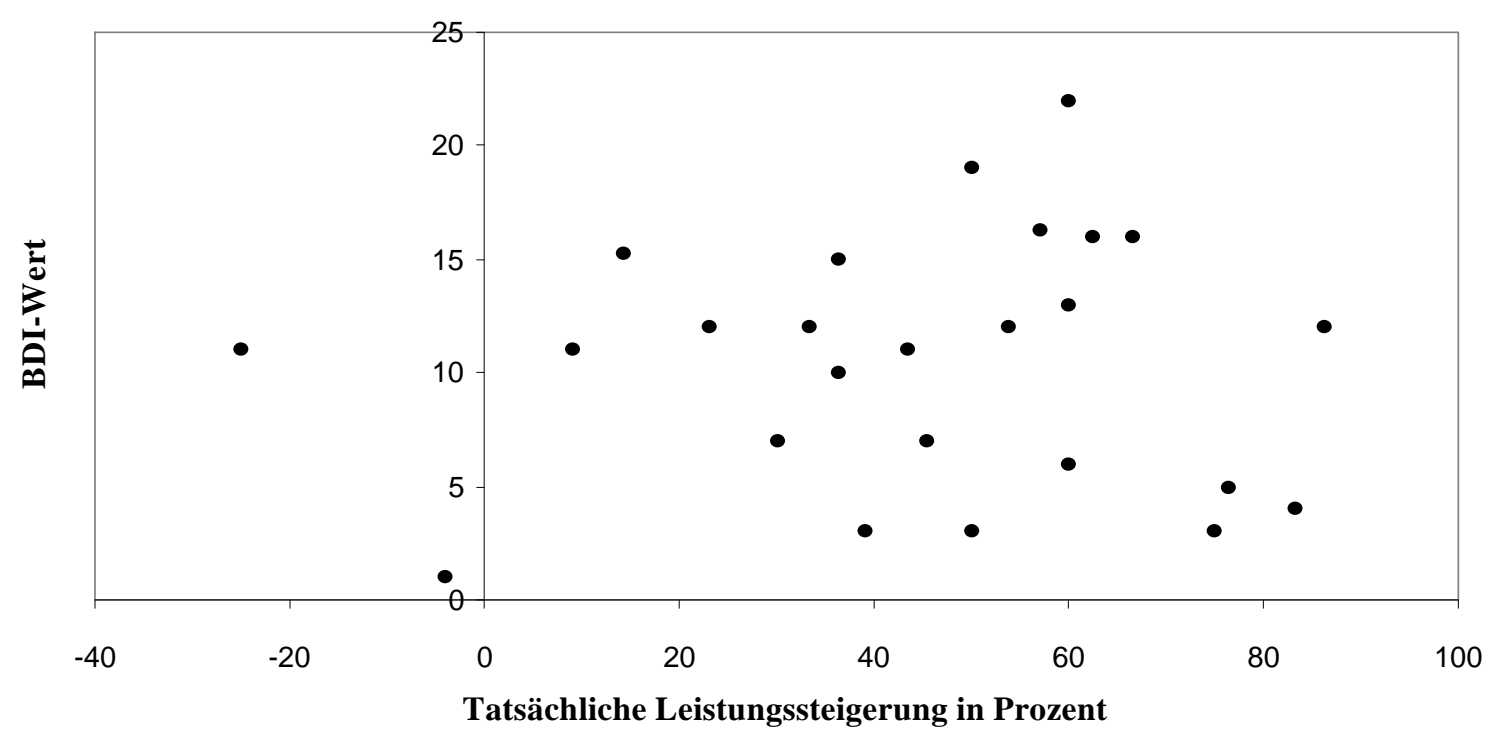

Abbildung 15: Tatsächlicher Kraftzuwachs in Abhängigkeit vom Grad der Depressivität

Die Korrelation zwischen dem Grad der Depressivität und der tatsächlichen Leistungssteigerung beträgt bei $\mathrm{n}=28$

$$
\mathbf{r}=-.31
$$

Die statistische Signifikanzprüfung erfolgt gemäß Bortz (1989, S. 261) mit Hilfe des tTests:

$$
t=\frac{r \cdot \sqrt{n-2}}{\sqrt{1-r^{2}}}=\frac{-0.3068 \cdot \sqrt{28-2}}{\sqrt{1-0.3068^{2}}}=-1.6436
$$

Der t-Wert, der für eine Signifikanz auf dem $5 \%$-Niveau $(\alpha=.05)$ erreicht werden

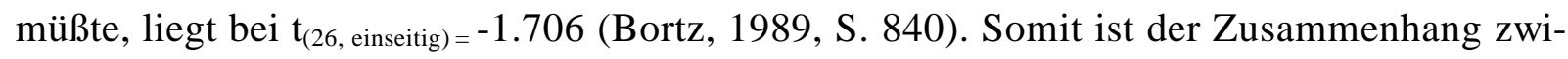
schen dem Depressionsgrad und der tatsächlichen Leistungssteigerung nicht signifikant.

Die $\mathrm{H}_{0,3-\text { Kraft }}$ wird beibehalten, die $\mathrm{H}_{1,3 \text {-Kraft }} \mathrm{muß}$ abgelehnt werden. 


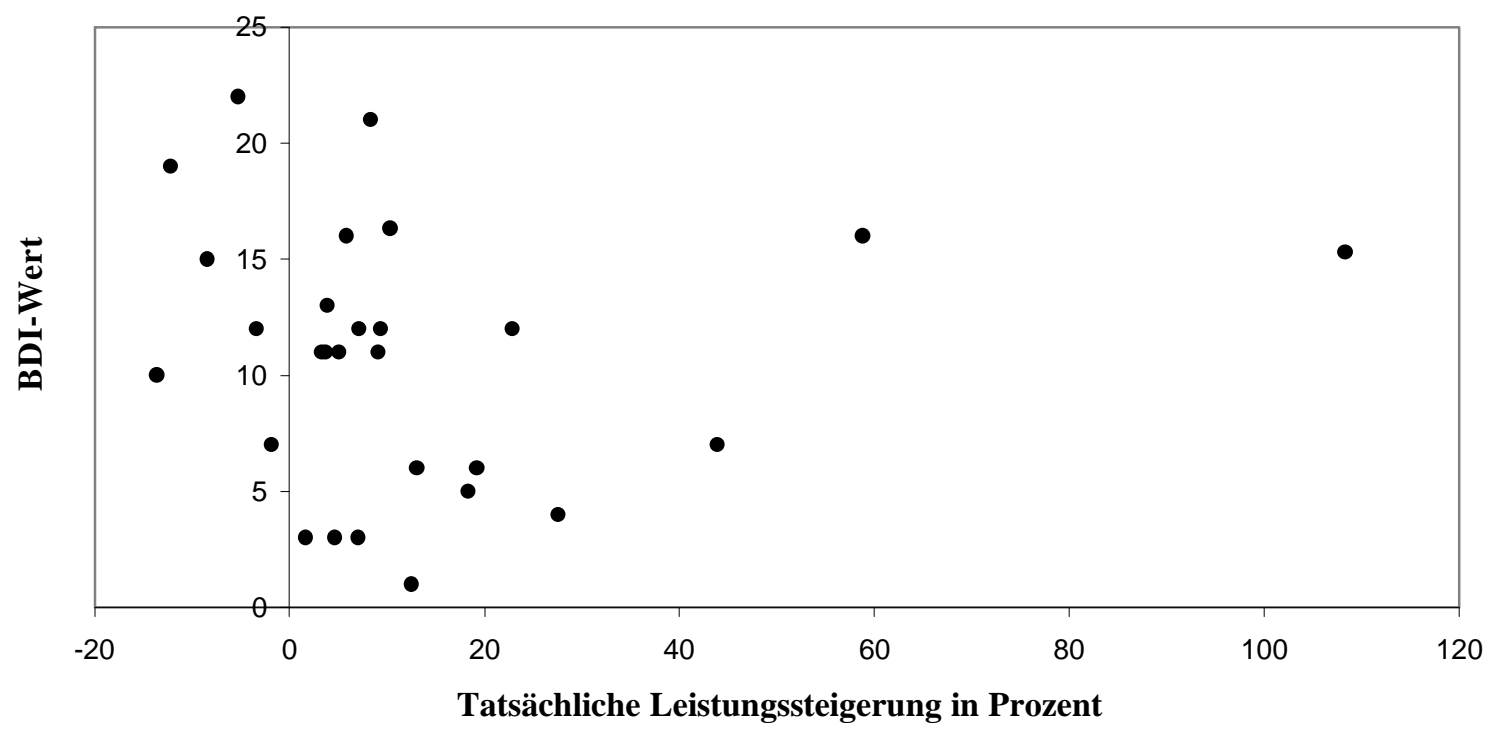

Abbildung 16: Tatsächlicher Schnelligkeitszuwachs in Abhängigkeit vom Grad der Depressivität

Die Korrelation zwischen dem Grad der Depressivität und der tatsächlichen Leistungssteigerung beträgt bei $n=28$

$$
\mathbf{r}=\mathbf{0 1}
$$

Die statistische Signifikanzprüfung erfolgt gemäß Bortz (1989, S. 261) mit Hilfe des tTests:

$$
\mathrm{t}=\frac{\mathrm{r} \cdot \sqrt{\mathrm{n}-2}}{\sqrt{1-\mathrm{r}^{2}}}=\frac{0.0056 \cdot \sqrt{28-2}}{\sqrt{1-0.0056^{2}}}=0.0286
$$

Der t-Wert, der für eine Signifikanz auf dem $5 \%$-Niveau $(\alpha=.05)$ erreicht werden müßte, liegt bei $t_{(26, \text { einseitig })=-1.706}$ (Bortz, 1989, S. 840). Somit ist der Zusammenhang zwischen dem Depressionsgrad und der tatsächlichen Leistungssteigerung nicht signifikant.

Die $\mathrm{H}_{0,3 \text {-Schnelligkeit }}$ wird beibehalten, die $\mathrm{H}_{1,3 \text {-Schnelligkeit }}$ muß abgelehnt werden. 


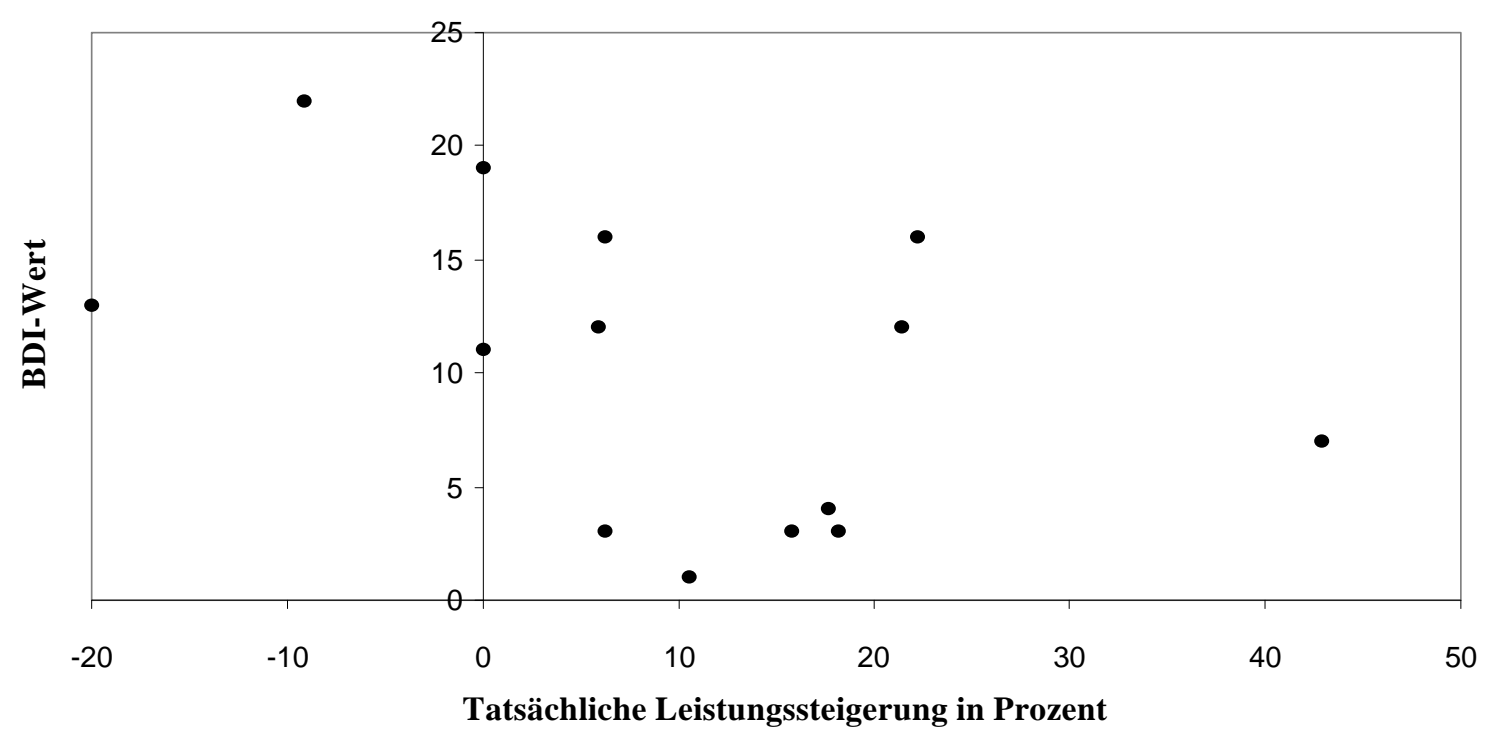

Abbildung 17: Tatsächlicher Ausdauerzuwachs in Abhängigkeit vom Grad der Depressivität

Die Korrelation zwischen dem Grad der Depressivität und und der tatsächlichen Leistungssteigerung beträgt bei $\mathrm{n}=16$

$$
\mathbf{r}=-.18
$$

Die statistische Signifikanzprüfung erfolgt gemäß Bortz (1989, S. 261) mit Hilfe des tTests:

$$
t=\frac{r \cdot \sqrt{n-2}}{\sqrt{1-r^{2}}}=\frac{-0.1770 \cdot \sqrt{16-2}}{\sqrt{1-0.1770^{2}}}=-0.6230
$$

Der t-Wert, der für eine Signifikanz auf dem $5 \%$-Niveau $(\alpha=.05)$ erreicht werden

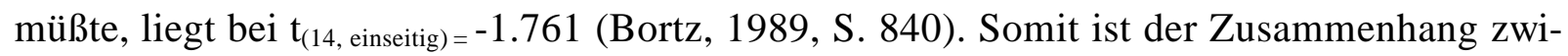
schen dem Depressionsgrad und der tatsächlichen Leistungssteigerung nicht signifikant.

Die $\mathrm{H}_{0,3 \text {-Ausdauer }}$ wird beibehalten, die $\mathrm{H}_{1,3 \text {-Ausdauer }}$ muß abgelehnt werden. 


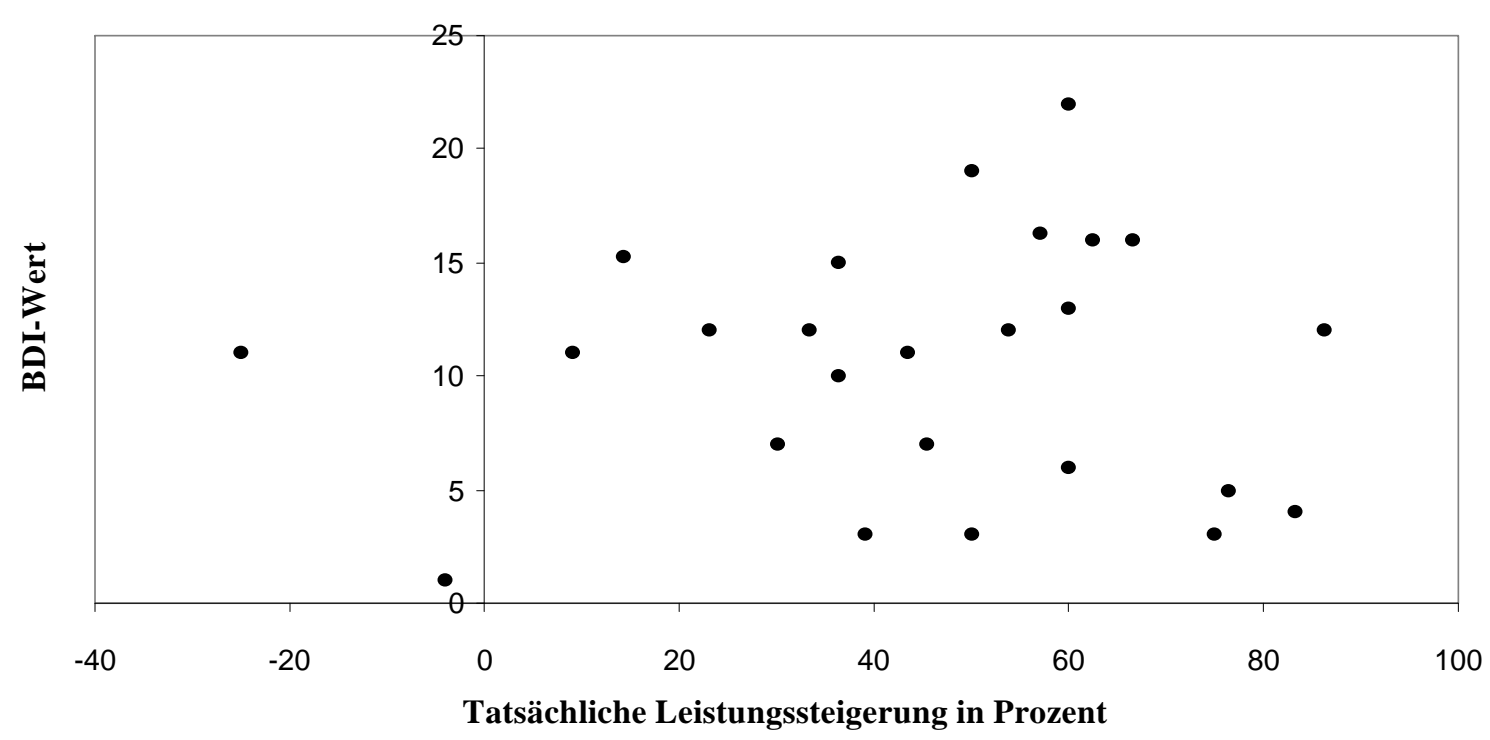

Abbildung 18: Tatsächlicher Koordination-I-Zuwachs in Abhängigkeit vom Grad der Depressivität

Die Korrelation zwischen dem Grad der Depressivität und und der tatsächlichen Leistungssteigerung beträgt bei $\mathrm{n}=25$

$$
\mathbf{r}=\mathbf{0 1}
$$

Die statistische Signifikanzprüfung erfolgt gemäß Bortz (1989, S. 261) mit Hilfe des tTests:

$$
\mathrm{t}=\frac{\mathrm{r} \cdot \sqrt{\mathrm{n}-2}}{\sqrt{1-\mathrm{r}^{2}}}=\frac{0.0009 \cdot \sqrt{25-2}}{\sqrt{1-0.0009^{2}}}=0.0432
$$

Der t-Wert, der für eine Signifikanz auf dem $5 \%$-Niveau $(\alpha=.05)$ erreicht werden

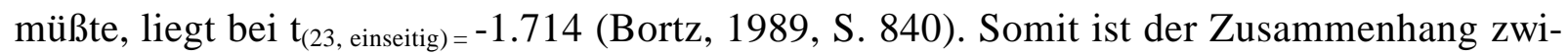
schen dem Depressionsgrad und der tatsächlichen Leistungssteigerung nicht signifikant.

Die $\mathrm{H}_{0,3 \text {-Koordination I }}$ wird beibehalten, die $\mathrm{H}_{1,3 \text {-Koordination I }}$ muß abgelehnt werden. 


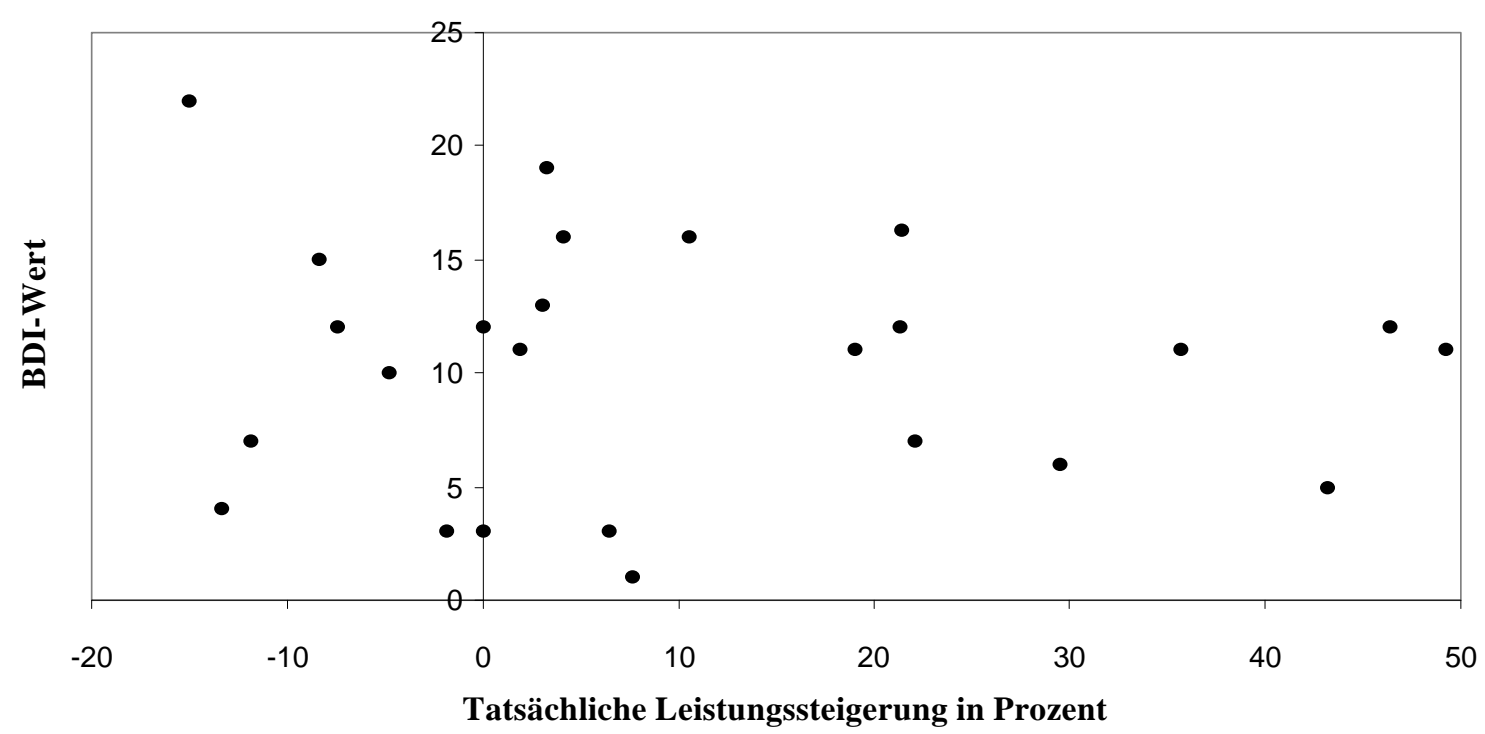

Abbildung 19: Tatsächlicher Koordination-II-Zuwachs in Abhängigkeit vom Grad der Depressivität

Die Korrelation zwischen dem Grad der Depressivität und und der tatsächlichen Leistungssteigerung beträgt bei $\mathrm{n}=25$

$$
\mathbf{r}=-.13
$$

Die statistische Signifikanzprüfung erfolgt gemäß Bortz (1989, S. 261) mit Hilfe des tTests:

$$
\mathrm{t}=\frac{\mathrm{r} \cdot \sqrt{\mathrm{n}-2}}{\sqrt{1-\mathrm{r}^{2}}}=\frac{-0.1257 \cdot \sqrt{25-2}}{\sqrt{1-0.1257^{2}}}=-0.6077
$$

Der t-Wert, der für eine Signifikanz auf dem $5 \%$-Niveau $(\alpha=.05)$ erreicht werden

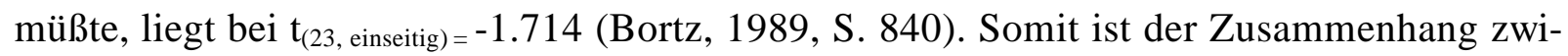
schen dem Depressionsgrad und der tatsächlichen Leistungssteigerung nicht signifikant.

Die $\mathrm{H}_{0,3 \text {-Koordination II }}$ wird beibehalten, die $\mathrm{H}_{1,3 \text {-Koordination II }} \mathrm{mu}$ abgelehnt werden. 


\subsection{Darstellung der $\mathbf{H} 4$}

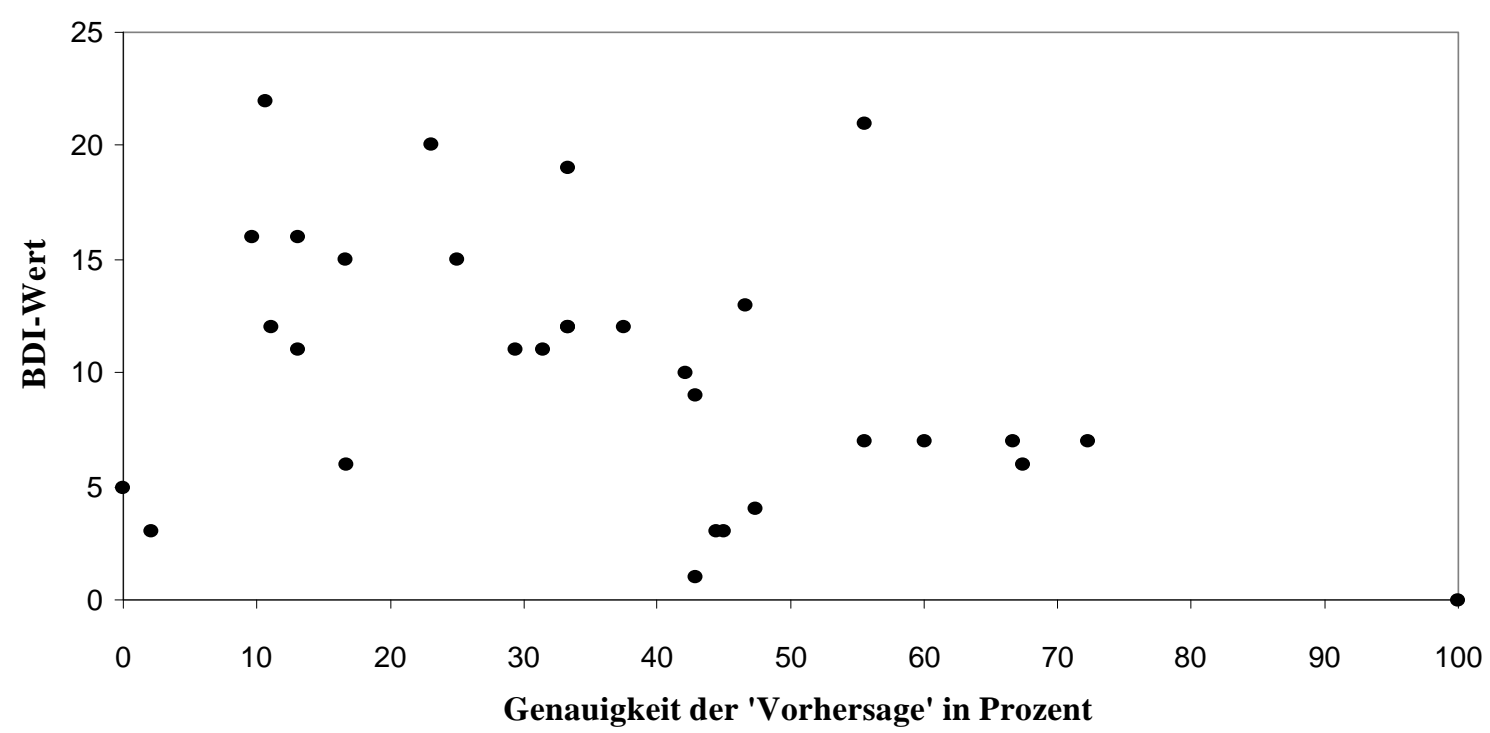

Abbildung 20: Genauigkeit der Krafteinschätzung in Abhängigkeit vom Grad der Depressivität

Die Korrelation zwischen dem Grad der Depressivität und der Genauigkeit Einschätzung beträgt bei $n=31$

$$
\mathbf{r}=\mathbf{- . 4 0} \text {. }
$$

Die statistische Signifikanzprüfung erfolgt gemäß Bortz (1989, S. 261) mit Hilfe des tTests:

$$
\mathrm{t}=\frac{\mathrm{r} \cdot \sqrt{\mathrm{n}-2}}{\sqrt{1-\mathrm{r}^{2}}}=\frac{-0.3958 \cdot \sqrt{31-2}}{\sqrt{1-0.3958^{2}}}=-2.3210
$$

Der t-Wert, der für eine Signifikanz auf dem $5 \%$-Niveau $(\alpha=.05)$ erreicht werden

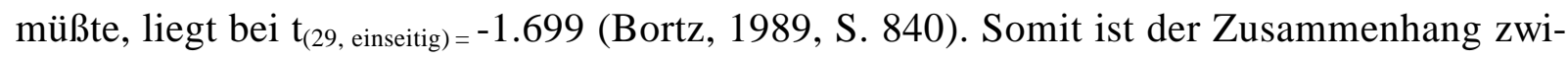
schen dem Depressionsgrad und der Genauigkeit der Einschätzung signifikant.

Die $\mathrm{H}_{0,4}$ wird verworfen, die $\mathrm{H}_{1,4}$ kann angenommen werden. 


\subsection{Darstellung weiterer Ergebnisse}

Der Mittelwert der Handkraft zum ersten Untersuchungszeitpunkt betrug $26.61 \mathrm{Kg}( \pm$ 11.03), zum zweiten Meßzeitpunkt 27.75 Kg ( \pm 10.62). Die geringe durchschnittliche Steigerung von $1.14 \mathrm{Kg}( \pm 4.89)$ läßt sich vermutlich auf die Verschlechterung einiger Patienten zurückführen. In der Bedingung, in der zuerst mit der Hälfte der antizipierten Maximalkraft zu drücken war, lag der Mittelwert bei $13.52 \mathrm{Kg}( \pm 8.04)$. Die durchschnittliche Vorhersage ging von einer Steigerung um $16.23 \%( \pm 14.68) 42$ auf $29.87 \mathrm{Kg}( \pm 11.63)$ aus.

Der Mittelwert in der Schnelligkeitsbedingung zum ersten Untersuchungszeitpunkt lag bei 49.46 Anschlägen ( \pm 18.61), zum zweiten Zeitpunkt wurden 53.64 Anschläge $( \pm 16.63)$ registriert. Die Steigerung betrug 4.95 Anschläge $( \pm$ 8.75). Die durchschnittliche Vorhersage erwartete eine Steigerung auf 57.70 Anschläge ( \pm 19.10), was einem Zuwachs von 21.19 $\%( \pm 24.10)$ entspricht.

In der Ausdauerbedingung wurde bei der ersten Erhebung eine durchschnittliche Leistung von 72.94 Watt $( \pm 19.13)$ gemessen. Diese steigerte sich um 7.24 Watt $( \pm 11.14)$ auf 80.94 Watt $( \pm$ 23.33). Die durchschnittliche Vorhersage entsprach einer Steigerung von $12.34 \%( \pm 11.97)$ auf 79.21 Watt $( \pm 19.56)$.

Die Fehlerzeit in der Koordination-I-Bedingung entsprach $22.26 \%( \pm 19.35)$. Sie verbesserte sich auf durchschnittlich $12.49 \%( \pm 13.91)$. Die Verbesserung betrug $9.15 \%( \pm$ 9.89). Vorhergesagt wurden in dieser Bedingung $13.30 \%$ ( \pm 13.99). Im Durchschnitt gingen die Patienten von einer Verbesserung um $41.77 \%( \pm 21.61)$ aus.

In der Koordination-II-Bedingung benötigten die Patienten $65.06 \mathrm{sec}( \pm 47.17)$ zum Eindrehen der drei Schrauben. Zur zweiten Untersuchung sank der Wert um $10.47 \mathrm{sec}( \pm$ $19.49)$ auf 54.25 sec $( \pm 34.35)$. Vorhergesagt wurde eine Verbesserung auf $49.06 \mathrm{sec}( \pm$ 31.61). Die prozentuale Leistungssteigerung lag bei $20.51 \mathrm{sec}( \pm 16.94)$.

Die Patienten wurden zusätzlich gebeten, ihren momentanen Leistungsstand einzuschätzen (vgl. S. 150). Dieser lag, unter Zugrundelegung eines prämorbiden Leistungsstands von

42 Mittelwert und Standardabweichung der individuellen, in Prozentwerte umgerechneten Vorhersagen 
$100 \%$, bei $52.30 \%( \pm 17.38)$. Für das Ende des Rehabilitationsaufenthaltes nahmen die Patienten eine Steigerung auf 70.26\% ( \pm 18.26) an. Die durchschnittliche Erwartung bezüglich der persönlichen Verbesserung lag bei einer Steigerung um $43.38 \%$ ( \pm 46.30$)$. Die Korrelation mit dem BDI-Wert betrug nur $\mathrm{r}=.04$, während der vorhergesagte Leistungsstand in absoluten Zahlen und die Depressionswerte mit $\mathrm{r}=-.37$ korrelierten. Der Zusammenhang zwischen der geschädigten Hemisphäre und dem Depressionswert war nicht signifikant $(\mathrm{r}=.16)$.

Die Korrelation der Ratingskala nach Boné, Ladurner und Pichler (1988; vgl. Kap. 3.3.2.8, S. 147) mit dem Grad der Depressivität beträgt $r=-.03$ (vgl. Anhang K, S. 232). Somit ist kein Zusammenhang zwischen dem Grad der Depressivität und dem Ausmaß der Schädigung vorhanden.

Um einen Einfluß der Seitigkeit auf die Genauigkeit auszuschließen, wurde die Korrelation zwischen der betroffenen (und demzufolge untersuchten) Seite und der Genauigkeit der Belastungswahrnehmung ermittelt. Sie beläuft sich auf $r=.00(r=-.0004)$. Der Einfluß der untersuchten Seite auf die Genauigkeit der Einschätzung kann damit als nicht vorhanden betrachtet werden. Ebenso hatten auch Alter und Geschlecht keinen systematischen Einfluß auf den Depressionswert. Die Korrelationen haben eine Höhe von $r=.04$ bzw. $r=-$ .08 .

Im Rahmen der 'self-fulfilling prophecy' (vgl. Beck, Rush, Shaw \& Emery, 1994, S. 294; siehe auch S. 181) ist abschließend der Zusammenhang zwischen der prozentualen Leistungssteigerungsvorhersage und der tatsächlichen Leistungssteigerung in Prozent von Interesse. Hierfür wurden die Wertereihen der Steigerung und die der Vorhersage miteinander korreliert. Die Höhe des Zusammenhangs betrug bei der Kraft $\mathrm{r}=.01$, hinsichtlich der Schnelligkeit und der Ausdauer $\mathrm{r}=.56$ bzw. $\mathrm{r}=-.46$. Bei der Koordination wurde ein $\mathrm{Zu}-$ sammenhang von $\mathrm{r}=.24$ (Koordination I) und $\mathrm{r}=.15$ (Koordination II) festgestellt. 


\section{Diskussion}

Die Bewährung der Theorie in einem neuen Kontext wird diskutiert. Die Aussagen des depressiven Realismus sind für Sportler aufgrund von zwei Überlegungen in hohem Maße relevant. Der Depressionsgrad und der Grad der ZNSSchädigung variieren nicht systematisch miteinander. Störvariablen werden diskutiert und Vorschläge für weitere Studien gemacht. Kraft ist in der unmittelbaren Wahrnehmung besser repräsentiert als die übrigen konditionellen und koordinat ven Fähigkeiten. Die Forschung des depressiven Realismus wird von den Gründerinnen aus verschiedenen Perspektiven betrachtet, wobei für die Ergebnisse der vorliegenden Arbeit die 'naive Sicht' von Interesse ist. Die Ergebnisse der zwèten und dritten Hypothese werden diskutiert. Weitere Implikationen beschäftigen sich mit der Überbelastung im Freizeitund Leistungssport, mit dem Ethos im Umfeld des Sports und allgemeiner mit dem Phänomen der Demoralisierung bzw. des 'Totalitarian Ego'. Es bleibt offen, wie sich das Paradigma des depressiven Realismus weiterentwickelt. Die motiætionalen Effekte jedoch, die aus der Verzerrung resultieren, sind vermutlich der Preis, der für die motivationale Bewälit gungsfähigkeit alltäglicher Probleme gezahlt werden muß

Nach der methodologischen Empfehlung Poppers - unter den „,... zur Diskussion stehenden Hypothesen die kühnste, nämlich die gehaltvollste...“ (Stegmüller, 1979, S. 110; kursiv im Original) zu wählen - wurde im Rahmen dieser Arbeit für die Vorhersage von Leistungssteigerung und die durch die Belastungswahrnehmung vermittelte Selbsteinschätzung gefordert, daß - indem alle Abweichungen vom Nullpunkt mit ihrem Betrag in die statistische Auswertung eingingen - Depressive sich im Gesamtausmaß der Werte geringer verzerrt einschätzen als nichtdepressive Personen. Die Realismushypothese wurde in einem Bereich überprüft, in dem bislang nocht keine derartigen Untersuchungen durchgeführt wurden. Die vorliegende Studie folgte dabei dem „Typ-b-Forschungsprogramm“ (Herrmann, 1976, S. 29; siehe auch 'Einleitung', Kap. 1, S. 9), indem sie vor dem theoretischen Hintergrund des depressiven Realismus untersuchte, ob sich dessen zentrale Aussagen in den Phänomenbereichen der Vorhersage körperlicher Leistungssteigerung bei der Wiederherstellung nach ZNS-Schädigung und der Selbsteinschätzung, vermittelt durch die Belastungswahrnehmung, bewähren; hieraus würde eine Erhöhung des empirischen Gehalts der Realismushypothese resultieren (für ein tieferes Verständnis der 'strukturalistischen Konzeption wissenschaftlicher Theorien' siehe Westermann, 1987, S. 26 ff.). Während sich die statistische und somit die sportpsychologische Hypothese in dem Bereich der Belastungswahrnehmung bestätigt hat (H 4; vgl. S. 168), sind in bezug auf die Vorhersagegenauigkeit (H 1; vgl. S. 152 ff.) die Befunde nicht einheitlich. In dem ersten Untersuchungsteil (Kraft) wurde die zugrundeliegende Hypothese bestätigt, in den anderen vier Versuchsteilen (Schnelligkeit, Ausdauer, Koordination I und Koordination II) waren keine signifikanten Zusammenhänge zu beobachten. Die übrigen Hypothesen (H 2 und $\mathrm{H}$ 3) waren nicht signifikant. Die Entscheidung über das Zutreffen bzw. das Nichtzutreffen der sportpsychologischen Hypothesen (vgl. Kap. 3.1, S. 134) wird hierbei in Analogie des Wegs der Hypothesenableitung, der von der sportpsychologischen Hypothese über die sportpsychologische Vorhersage zur statistischen Hypothese führte, gefällt, indem dieser Weg in umgekehrter Richtung begangen wird. Aus dem Erreichen oder Verfehlen des Si- 
gnifikanzniveaus der statistischen Hypothesen wird die sportpsychologische Vorhersage als erfüllt bzw. nicht erfüllt beurteilt, woraus sich danach die Annahme bzw. die Ablehnung der sportpsychologischen Hypothesen ergibt.

Die Erhöhung des empirischen Gehalts der Realismushypothese (s. o.) bezieht sich in erster Linie auf den Bereich der motorischen Leistungssteigerung bzw. der Belastungswahrnehmung bei neurologischen Patienten. Eine Übertragung auf andere Bereiche der motorischen Leistungsfähigkeit im allgemeinen oder dem Freizeit- bzw. Leistungssport im speziellen (siehe S. 183 ff.) folgt zunächst einer Plausibilitätsheuristik. „Für die Verallgemeinerung einer Korrelation auf eine Grundgesamtheit ist zu fordern, daß die untersuchte Stichprobe tatsächlich zufällig gezogen wurde und keine irgendwie geartete, systematische Selektion darstellt“ (Bortz, 1989, S. 267; kursiv im Original). Da sich die Theorie der Realismushypothese in der vorliegenden Arbeit zunächst im Rahmen einer systematisch selektierten Stichprobe bewähren sollte (neurologische Rehabilitation), kann eine letztendliche, empirisch abgesicherte Übertragung erst erfolgen, wenn entsprechende Folgestudien in den jeweiligen Bereichen realisiert wurden. Es ist Aufgabe weiterer Untersuchungen zu klären, inwieweit das Phänomen des depressiven Realismus auch auf diesen Gebieten zu finden ist. Diese Untersuchungen sollten sich mit dem Problem des depressiven Realismus beispielsweise im mehr oder weniger leistungsorientierten Freizeitsport, im Spitzensport, im Schulsport, bei der Rehabilitation orthopädischer Patienten oder auch im Rehabilitations- und Behindertensport allgemein befassen. Variationen über Alter, Geschlecht, soziodemographische Daten oder Persönlichkeitseigenschaften müßten dabei in einzelnen Arbeiten isoliert betrachtet werden.

Die Tatsache, daß auch mit den statistisch signifkanten Korrelationskoeffizienten nur ein vergleichsweise kleiner Bereich der Varianz aufgeklärt wurde, liegt vermutlich in dem Einfluß begründet, den die sogenannten Störvariablen ausübten. „Dem Varianzanteil, der [in varianzanalytischen Verfahren] auf den Treatmentstufen beruht, steht ein restlicher Varianzanteil gegenüber, der vom Treatment unabhängig ist und der auf andere, die Meßwerte beeinflussende Variablen wie z.B. unterschiedliche Motivation, unterschiedliche Sprachbegabung, Meßungenauigkeiten usw. zurückzuführen ist. Diesen restlichen Varianzanteil bezeichnen wir zusammenfassend als Fehlervarianzanteil .... Diejenigen Variablen, die die Größe des Fehlervarianzanteils bestimmen, bezeichnen wir zusammenfassend als 'Störvariablen'“ (Bortz, 1989, S. 307; Hervorhebung im Original). Der erste zu besprechende Faktor ist die Seitigkeit. Auch wenn es keinen systematischen Einfluß der betroffenen und aus diesem Grund auch untersuchten Seite gab (der Zusammenhang zwischen der 
Genauigkeit der durch die Belastungswahrnehmung vermittelten Selbsteinschätzung und der Seitigkeit lag bei $\mathrm{r}=-.0004)$, kann die unterschiedliche Verteilung der betroffenen Seite die aufgeklärte Varianz verringert haben. In nachfolgenden Untersuchungen, in denen die Meßgröße nicht unabdingbar von der geschädigten Hemisphäre abhängig ist, sollte dieser Faktor konstant gehalten werden. Eine Möglichkeit wäre, die Untersuchung auf der jeweiligen Seite der Händigkeit durchzuführen. Da aber die Hemisphärendominanz bei Linkshändern nicht so eindeutig ist (vgl. Hartje \& Poek, 1997), sollte eine derartige Studie nur mit Rechtshändern durchgeführt werden. Das Alter der Patienten war bei der vorliegenden Untersuchung vergleichsweise hoch, da bei vielen der beteiligten Personen ein Z. n. Apoplexia cerebri diagnostiziert worden war, und die Anfälligkeit für diese Krankheit mit dem Alter steigt (vgl. Mäurer \& Diener, 1996). Auch hier kann ein systematischer Einfluß ausgeschlossen werden, da das Alter mit der Prädiktorvariable 'Depression' mit nur $\mathrm{r}=.04$ korrelierte. Das Geschlecht, das in der Höhe von $r=-.08$ mit dem Grad der Depressivität zusammenhing, wirkte sich ebenfalls nicht systematisch auf die untersuchten Zusammenhänge aus. Vor allem diese beiden Variablen sollten jedoch in Folgestudien isoliert betrachtet werden, da sich ein großer Anteil der nicht aufgeklärten Varianz vermutlich auf diese Faktoren zurückführen läßt. Das Ausmaß der Vorerfahrung in der Selbsteinschätzung spielt möglicherweise zusätzlich eine große Rolle. Haben die in eine Untersuchung einbezogenen Personen schon viel Erfahrung in der Selbsteinschätzung, wird die Gesamtvariation geringer ausfallen und somit die Zielgröße genauer abgebildet werden. Ein weiterer Weg, die Variationsbreite der Werte zu verringern, würde dann beschritten werden, wenn sportliche Leistungssteigerungen im Mittelpunkt einer Studie stehen würden. Die Erfahrung, in welchem Maße sich die eigene Leistung verändert, ist naturgegeben bei 'normalen' Leistungssteigerungen viel größer als bei solchen, die aufgrund einer ZNS-Schädigung notwendig werden. Patienten erleben einen derartigen Rehabilitationsprozeß in der Regel zum ersten Mal und haben nur wenig Erfahrung in der Einschätzung der Erholungsmöglichkeiten ihrer motorischen Leistungsfähigkeit.

Nachdem im Kap. 2.3.1.5 (S. 84) Depression als Folge einer ZNS-Schädigung besprochen wurde, liegt die Vermutung nahe, daß mit der Schwere der ZNS-Schädigung der Grad der Depressivität zunimmt. Unter Zugrundelegung dieser Annahme wäre eine Eindeutigkeit der Ergebnisse (Kap. 4, S. 152) nicht mehr gegeben, da die Prädiktorvariable 'Depression' mit dem Ausmaß der Schädigung konfundieren würde und somit die Kriteriumsvariable 'Genauigkeit der Einschätzung' nicht mehr eindeutig der Prädiktorvariable 'Depression' zuzuordnen wäre. Gegen die Annahme, daß der Schweregrad systematisch mit dem Grad 
der Depressivität variiert, sprechen jedoch sowohl die einschlägige Fachliteratur als auch Befunde der vorliegenden Arbeit.

Verschiedene Studien zeigten, daß das Ausmaß der Schädigung nicht mit dem Grad der Depressivität zusammenhängt. In einer Studie, die sich mit der Beziehung zwischen depressiven Störungen nach Hirninfarkt und Infarktlage, Hirnatrophie und kognitiven Defiziten befaßte, stellten Zerfaß, Kretzschmar und Förstl (1992) fest, daß ,... sich in einer Regressionsanalyse kein signifikanter Zusammenhang zwischen dem Depressions Score [sic] und Geschlecht, Alter, Zeitintervall seit dem Infarkt, körperlicher Behinderung und Ventrikelweite (,ventricle-brain ratio“) nachweisen ließ. Darüber hinaus wurden die kombinierten Effekte von Lateralität, sagittaler Position und Infarktgröße untersucht; sie erwiesen sich als nicht relevant“ (Zerfaß, Kretschmar \& Förstl, 1992, S. 165). Auch in einer Studie von Herrmann, Bartels, Schumacher und Wallesch (1995) wurde kein derartiger Zusammenhang gefunden. „We found no significant correlation between the anteriority of lesion...., lesion volume..., or VBR [Ventricle-to-Brain Ratio] and CDS [Depressionswert] sum scores“ (Herrmann, Bartels, Schumacher \& Wallesch, 1995, S. 851). In bezug auf andere Studien berichteten sie, daß ,... pathoanatomic parameters such as volume of lesions ... showed no clear-cut association with type or severity of depressive disorders" (Herrmann, Bartels, Schumacher \& Wallesch, 1995, S. 850). Hinsichtlich der eigenen Studie diskutierten sie abschließend: „The results of all correlations between degree of depression and phathoanatomic measurements in the present study indicate that the occurrence of depressive disorders after stroke reflects neither a pure 'left frontal pathology' [vgl. Exkurs zur Depression nach ZNS-Schädigung, S. 84] nor a simple volume effect of the brain tissue damaged (a. a. O., S. 854). Auch Kallert und Krebs (1995) kamen nach einer eigenen Untersuchung und der Sichtung der einschlägigen Literatur zu keinem eindeutigen Schluß: „Letztlich müssen derzeit die Effekte der Läsionslokalisation und des -volumens ... als nicht ausreichend geklärt beurteilt werden“ (Kallert \& Krebs, 1995, S. 12). In der Literatur wird in Anlehnung an das WHO-Klassifikationssystem der Behinderung (ICIDH) aufgrund der Unterscheidung von 'Impairment', 'Disability' und 'Handicap' das Ausmaß der Schädigung auf den verschiedenen Ebenen beschrieben. Dabei enstspricht 'Impairment' der neurologischen Schädigung als solcher, 'Disability' der Fähigkeitsstörung und 'Handicap' der (sozialen) Beeinträchtigung (Schupp, 1996, S. 117). In der vorliegenden Arbeit wurde das Ausmaß der Schädigung nach der Ratingskala von Boné, Ladurner und Pichler (1988) eingeschätzt, die sowohl die Ebene der 'Disability' als auch die des 'Handicaps' berücksichtigt. Die Korrelation der Ratingskala mit dem Grad der Depressivität beträgt $\mathrm{r}=-.03$ (vgl. Anhang K, S. 232). Demnach scheint keine Variablenkonfusion von Depression und ZNS- 
Schädigung als systematisch verzerrender Einfluß in der vorliegenden Arbeit vorhanden zu sein.

Da dem Sporttreiben eine positive Wirkung auf die Depressivität nachgesagt wird (vgl. Exkurs I: Sport und Depression, Kap. 2.3.1.4, S. 81), könnte vermutet werden, daß unter Sportlern das Problem der Depression kaum vorhanden ist. Damit wären Aussagen zu den Implikationen der Wirkung von Depressivität auf Selbsteinschätzung und Vorhersage von Leistungssteigerung im Sportbereich gegenstandslos. Gegen diese Annahme sprechen zwei Überlegungen.

In den vorgestellten Studien (Kap. 2.3.2.3, S. 93 ff.) waren zumeist Personen mit lediglich subklinischen Depressionen involviert. Die Operationalisierung der subklinischen Depression erfolgte in der Regel über einen BDI-Wert ab 9 (vgl. Fußnote 18, S. 95), während von einer leichten klinischen Depression erst ab einem BDI-Wert von etwa 14 ausgegangen werden kann (vgl. Kap. 3.3.2.7, S. 145). Die Aussagen über Depressive beziehen sich demnach immer auch auf 'subklinisch depressive Personen'. In einer Untersuchung von Sportstudenten zeigte sich, daß die Studenten einen durchschnittlichen D-S ${ }^{433}$-Wert von 9.4 ( \pm 5.7) hatten (Krause, 1995), womit dieser im Vergleich zu dem für eine Durchschnittsbevölkerung als Norm veröffentlichte Wert von 7.09 ( \pm 5.47; $\mathrm{N}=1700$; Zerssen, 1986, S. 276) sogar leicht erhöht war. In einer Forschungsarbeit mit dem Freiburger Persönlichkeitsinven$\operatorname{tar}\left(\mathrm{FPI}^{44}\right)$, auf dessen Subskalen auch Depressivität lädt, fand Sack (1975), der mehr als dreihundert Nichtsportler, Nichtwettkampfsportler und Wettkampfsportler miteinander verglich, daß ,... die Gruppendifferenzen ... zu gering [waren], um signifikante F-Werte ( $\mathrm{p} \leq$

\footnotetext{
${ }^{43}$ Die D-S' entstammt dem Psychiatrischen Informations-System (PSYCHIS, München), dessen Datenbank „,... contains questionnaire data on more than 3500 psychiatric inpatients, well over 700 medical patients, and approximately 4000 subjects from the general population investigated in epidemiological studies ..." (Zerrssen, 1986, S. 270). Sie besteht aus 16 Dimensionen. Die Auswertung erfolgt durch die Summation der Punkte pro Item, die von null bis drei reichen können. Nach Grubitzsch (1991) beträgt die ParalleltestReliabilität bei einer Eichstichprobe von $\mathrm{N}=1600 \mathrm{r}=.76$, bei Klinikpatienten $\mathrm{r}=.85$ (S. 520); Brickenkamp spricht von Werten zwischen 0.58 und 0.81 (1983, S. 908) und gibt die Validität in Form einer Korrelation der Depressions-Skalen mit der Zugehörigkeit zu verschiedenen psychiatrischen Gruppen und Kontrollgruppen mit $\mathrm{r}=.72$ an (ebd.).

${ }^{44}$ Das FPI-R als ,,... a general personality inventory similar to the Minnesota Personality Inventory (MMPI)“ (Fähdrich, Helmchen \& Linden, 1986, S. 5), ist die 1984 erschienene, überarbeitete Fassung des 1978 von Fahrenberg veröffentlichten Persönlickeitsinventars (Rauchfleisch, 1994, S. 152; s. auch Fahrenberg, Hampel \& Selg, 1984). Es gehört zu den subjektiven Persönlichkeitstests (Brickenkamp, 1975, S. 15) und wurde faktoranalytisch mit dem Ziel der ,... mehrdimensionalen Erfassung individueller Persönlichkeitsstrukturen“ (Hollmann, 1988, S. 277) konstruiert. Es umfaßt 138 Items mit den Dimensionen Lebenszufriedenheit, Soziale Orientierung, Leistungsorientierung, Gehemmtheit, Erregbarkeit, Aggressivität, Beanspruchung, Körperliche Beschwerden, Gesundheitssorgen, Offenheit, Extraversion und Emotionalität. Als „... Reliabilitätsmaß werden von den Autoren die Konsistenzkoeffizienten a für die einzelnen Skalen angegeben. Sie reichen von 0.71 bis 0.84 mit einer mittleren Konsistenz von 0.77 ..." (Hollmann, 1988, S. 281; für eine ausführliche Übersicht siehe Fahrenberg, Hampel \& Selg, 1984, S. 49 ff.).
} 
.05) zu produzieren“ (S. 102). Demzufolge unterscheidet sich die Häufigkeitsverteilung subklinisch Depressiver nicht innerhalb der Gruppen 'Sportler' und 'Nichtsportler'.

Da man bislang von einem größeren Realitätssinn bei Nichtdepressiven ausgegangen war, treten die Depressiven nun aus dem Blickwinkel des depressiven Realismus ihre Nachfolge als 'realistischere Personen' an. Dies hat für die Sicht dieser Personen höchstens eine Reduktion negativer Implikationen zur Folge, die aufgrund des bisherigen Verständnisses von Depression bestanden. Sie können nicht mehr als negativ verzerrte, unrealistische und als abseits der Wirklichkeit stehende Personen gesehen werden. Veränderungen kristallisieren sich aber für die Nichtdepressiven heraus, deren Wahrnehmungsprozessen auch aufgrund der vorliegenden Befunde (vgl. Kap. 2.3.2.3, S. 93) eine positive Verzerrung zugrundegelegt wird.

Durch die Tatsache, daß die Werte innerhalb der $\mathrm{H} 1_{\text {Kraft }}$ und $\mathrm{H} 4$ auf der statistischen Ebene das Signifikanzniveau erreicht haben, wird für die Ebene der sportpsychologischen Hypothesen und somit für die inhaltliche Ebene deutlich, daß in dem Bereich der Kraft sowohl die Vorhersage von Leistungssteigerungen als auch die Selbsteinschätzung, vermittelt durch die unmittelbare Belastungswahrnehmung, in Abhängigkeit vom Grad der Depressivität realistischer wird $(r=-.36$ und $r=-.40)$. In den übrigen Vorhersagebedingungen hingegen sind keine Zusammenhänge zwischen Depression und Einschätzungsgenauigkeit zu erkennen. Da jedoch keine gegenläufige Tendenz vorhanden war ${ }^{45}$, liegt die Vermutung nahe, daß auf den übrigen Gebieten der Vorhersage von Leistungssteigerung die Depression keinen differenzierenden Faktor darstellt. Da die Realismushypothese bislang nicht in diesem Kontext überprüft wurde, kann sich der Versuch einer Erklärung nicht an anderen, vergleichbaren Befunden orientieren.

Der Grund für die gesonderte Rolle der Kraft innerhalb der konditionellen Fähigkeiten kann in dem physiologisch gebahnten Zugang der Psyche zu der Beanspruchung bei Kraftleistungen liegen. Rezeptoren in den Muskeln und Sehnen melden jede Spannung und Spannungsänderung direkt an die höheren ZNS-Strukturen und ermöglichen damit eine unmittelbare mentale Repräsentation der Beanspruchung. Aus der Sicht des Parallelismus, nach dem Geist und Körper sich zueinander wie die Innen- und Außenfläche einer Kugel verhalten (vgl. Kap. 2.2.2.2, S. 58), entsprechen Kraftleistung und Kraftempfindung so dem

\footnotetext{
${ }^{45}$ Mit Ausnahme der Schnelligkeit, die mit einer Korrelation von $r=.30$ zwar nicht signifikant wäre, aber doch deutlich gegen die in der Hypothese angenommenen Richtung tendiert. Die Höhe ist aber vermutlich auf die starke Anfälligkeit von Korrelationen für Extremwerte zurückzuführen (Bortz, 1989, S. 269; vgl. Abbildung 6, S. 154: Zwei Werte liegen im rechten, oberen Bereich).
} 
gleichen Phänomen aus zwei Blickwinkeln. Bei der Ausdauer wird die Beanspruchung nur verzögert und mittelbar wahrgenommen, da nicht die Beanspruchung als solche, sondern die Anpassungsreaktionen des Organismus auf diese wahrgenommen werden können (vgl. Kap. 2.2, S. 55). Noch mittelbarer ist der Zugang zur Schnelligkeit, der nicht aufgrund einer Wahrnehmung oder einer unmittelbaren Erfahrung, sondern aufgrund der eigenen, selbstbezogenen Beobachtung erschlossen werden muß. Die koordinativen Leistungen haben schließlich keinerlei Entsprechung in der unmittelbaren Wahrnehmung, die Genauigkeit in der Vorhersage ihrer Leistungssteigerung korreliert folglich mit dem Depressionsgrad am geringsten $(r=.08$ und $r=-.05)$.

Möglicherweise aufgrund der unmittelbaren Wahrnehmung der Kraft folgte die Vorhersagegenauigkeit der Leistungssteigerung in diesem Bereich der Vorhersage der zugrundeliegenden Hypothese ( $\left.\mathrm{H}_{\mathrm{Kraft}}\right)$, während die Zusammenhänge zwischen Schnelligkeit, Ausdauer, Koordination I und Koordination II und dem Grad der Depressivität unsystematisch waren. Diese unmittelbare Wahrnehmung der Kraft ist vermutlich auch der Grund, warum drei der vier klassischen Gebiete der Belastungswahrnehmung die Wahrnehmung der Kraft zur Grundlage haben (vgl. Kap. 2.2.2.2, S. 58). Die Hypothese des depressiven Realismus hat sich dort bestätigt, wo nicht andere Faktoren für eine zu große, eventuell vorhandene Tendenzen überlagernde, unsystematische Variation verantwortlich waren (s. o.).

Nach etwa einem Jahrzehnt der Forschung auf dem Gebiet der von ihnen erstmalig postulierten 'depressive realism hypothesis' (Alloy \& Abramson, 1979) betrachteten Alloy und Abramson (1988) die Implikationen der Befunde aus einer von ihnen als ironisch, komisch, tragisch und naiv bezeichneten Perspektive.

Nach der 'ironischen' Perspektive ,... nondepressives' optimistic errors and biases in the laboratory may be indicative of rationality in everday life, while depressives' relative insusceptibility to such errors and biases may reflect irrationality over the long run" (Alloy \& Abramson, 1988, S. 253). Wenn Nichtdepressive in ihren alltäglichen Erfahrungen tatsächlich einen engen Zusammenhang zwischen ihren Handlungen einerseits, und Wirkungen in der (sozialen) Umwelt, Erfolg in vielen Situationen und positiven, auf die Person bezogenen Rückmeldungen andererseits erfahren, entspricht es einer nachvollziehbaren Heuristik, von diesem Zusammenhang auch in den Laborexperimenten auszugehen. Diese Sichtweise ist mit bestimmten Aspekten in der Wahrnehmungspsychologie vergleichbar. Der Umstand, daß sehr viele Personen unter Laborbedingungen beispielsweise optischen Täuschungen erliegen, ist kein Indiz dafür, daß sie auch im tatsächlichen Leben 'falsch' wahrnehmen. Die unangemessene Anwendung einer visuellen Strategie, die normalerweise 
in der physikalischen Welt gerade zu den richtigen Wahrnehmungen führt, kann unter manipulierten Bedingungen diese Täuschung hervorrufen. Die Depressiven sind entsprechend dieser Sicht in den komplexen Zusammenhängen der wirklichen Welt durch einen Mangel an Strategien zur Selbsteinschätzung gekennzeichnet. Dadurch kann es nicht zu einer Anwendung von alltäglichen Heuristiken auf die Laborsituation kommen. Die Depressiven sind, ironischerweise, realistischer. Für die Ergebnisse der vorliegenden Arbeit kann diese Sicht jedoch nicht gültig sein, da sie ihre Ergebnisse in der 'tatsächlichen Welt' gewonnen wurden, indem experimentell von den Versuchspersonen eine Vorhersage bzw. Selbsteinschätzung vor dem Hintergrund ihrer eigenen, alltagsbezogenen Einschätzung gefordert wurde. Somit findet in der Interpretation dieser Ergebnisse keine fälschliche Übertragung von einer künstlichen Laborsituation in das 'tatsächliche Leben' statt.

Die 'komische' Perspektive entspricht weitestgehend der Sicht der 'self-fulfilling prophecy' (siehe S. 181), nach der weder Depressive noch Nichtdepressive verzerrt wahrnehmen. Beide Gruppen verhalten sich ihrer Erwartung entsprechend und erfahren somit tatsächlich das Erwartete; ,... it is such negative self-schemata that lead to dysfunctional behaviors, which end up realistically confirming the negative schemata“ (Alloy \& Abramson, 1988, S. 255). Da sich die Wirkung der Vorhersage - auch unabhängig von dem Grad der Depressivität - bei den vorliegenden Ergebnissen lediglich in einer Versuchsbedingung bestätigt hat (vgl. S. 170), kann diese Sichtweise nicht der Interpretation aller Ergebnisse zugrundegelegt werden.

Als 'tragisch' wird die Sichtweise bezeichnet, nach der die Theorie von Beck (Beck, Rush, Shaw \& Emery, 1994; vgl. auch S. 76) in bezug auf die verzerrte Wahrnehmung Depressiver zutrifft, nicht aber auf den Realitätssinn nichtdepressiver Personen. Aus diesem Blickwinkel betrachtet unterliegen beide Gruppen entweder einer negativen oder einer positiven Verzerrung. „The tragic perspective could obtain if both groups engage in schemadriven or motivationally based biases of opposite content that are inconsistent with the environmental feedback each group typically confronts“ (Alloy \& Abramson, 1988, S. 255; vgl. auch Dykman, Abramson, Alloy \& Hartlage, 1989). Die Ergebnisse der vorliegenden Arbeit spiegeln zum einen eine einseitige (Nichtdepressive in den Hypothesen $\mathrm{H} 1_{\text {Kraft }}$ und H 4), zum anderen eine unsystematische Verzerrung wider. Im Bereich der Belastungswahrnehmung haben die Depressiven größeren Realitätssinn bewiesen, während sich in dem Bereich der Vorhersage von Leistungssteigerungen entweder die gleiche Befundlage ( $\left.\mathrm{H} 1_{\text {Kraft }}\right)$ fand, oder es war nicht einmal dann ein Zusammenhang zwischen Depression und Genauigkeit vorhanden, wenn anstatt des Betrags der Werte auch die negativen Werte in die Korrelationsrechnungen eingingen (Schnelligkeit: $r=.20$, Ausdauer: $r=.05$, Koordination 
I: $r=.17$ und Koordination II: $r=.01$ ). Ein enger Zusammenhang wäre jedoch zu erwarten, wenn sich Personen in ihrer Einschätzungsgenauigkeit in Abhängigkeit vom Grad der Depressivität entlang des gesamten Spektrums von 'negativ verzerrt' bis 'postiv verzerrt' bewegen würden. Somit ist auch die 'tragische' Perspektive für die vorliegenden Ergebnisse nicht zureichend.

Entsprechend der 'naiven' Sicht werden die Ergebnisse aus den Untersuchungen, die unter Laborbedingungen gemacht wurden, direkt auf das reale Leben übertragen. Demgemäß haben depressive Personen die meiste Zeit über realistische Gedanken und sind frei von einer verzerrenden Tendenz, während nichtdepressive Personen auf die Realität bezogen zu optimistisch leben: „Depressive rationality and nondepressive irrationality would be the case if any one or a combination of the motivational and cognitive mechanisms underlying depressive and nondepressive interference in the empirical studies is also operative in the everyday environment" (Alloy \& Abramson, 1988, S. 252). Die Perspektive wurde 'naiv' genannt, da in der Geschichte der Psychologie vielfältig belegt wurde, daß die Laborrealität nicht der alltäglichen gleicht (Davison \& Neale, 1996). In der vorliegenden Arbeit handelt es sich jedoch nicht um eine Übertragung von experimentellen Laborergebnissen auf eine oft anders beschaffene 'Wirklichkeit', sondern um Befunde, die in genau dieser Wirklichkeit erhalten wurden. Somit wird die Sicht der 'naiven Perspektive', daß depressive Personen die meiste Zeit über realistische Gedanken haben und frei von einer verzerrenden Tendenz sind, während nichtdepressive Personen auf die Realität bezogen zu optimistisch leben, den Aussagen der vorliegenden Arbeit gerecht.

In der Diskussion des depressiven Realismus (Kap. 2.3.2.4, S. 124) wurde bereits deutlich, daß jedoch eine 'naive' Übertragung nicht einer allgemeingültigen Übertragung entsprechen kann. Es zeigte sich, daß der depressive Realismus weder im Labor noch in der Feldforschung eine einheitliche Befundlage hervorbrachte, wenn differenzierende Faktoren eingeführt wurden: „However, to capture the essence of depressive cognitive processes, it may be essential to broaden our perspective beyond naive realism, beyond the simple question of whether depressive individuals are realistic. How do they operate on their perceptions of the world?" (Dobson \& Fanche, 1989, S. 429).

Die zweite Hypothese (H 2) ging davon aus, daß ,... in accord with the view that depressed persons are characterized by a sense of personal incompetence ... " (Golin, Terrell \& Johnson, 1977, S.441) die Höhe der vorhergesagten Leistungssteigerung zum Zeitpunkt $\mathrm{t}_{1}$ im reziproken Verhältnis zum Depressionswert steht. Dies konnte in keinem der Bereiche verifiziert werden. Ein beachtenswerter Zusammenhang war nur in bezug auf die Ausdauer 
feststellbar, der aber dem hypothesenkonträren Trend folgte. Die Korrelation zwischen der vorhergesagten Ausdauersteigerung und den BDI-Werten lag bei $r=.54$. Damit erwarteten Depressive eine besonders große Leistungssteigerung, was im deutlichen Gegensatz zu ihrer tatsächlichen Leistungssteigerung stand, die mit $\mathrm{r}=-.17$ erwartungsgemäß negativ mit dem Grad der Depressivität korrelierte (s. u.). Da die Diskrepanz dieser Zusammenhänge selbst bei der geringen Personenzahl in diesen Versuchsbedingungen nicht ignoriert werden kann, muß eine Interpretation beiden Tatbeständen gerecht werden. Eine Deutung kann aus der Sicht der Reaktanz-Theorie vorgenommen werden (vgl. auch S. 102). „Glaubt eine Person, sich in einer bestimmten Situation grundsätzlich frei verhalten zu können, und erlebt dann eine Einengung, so daß die Freiheit geringer wird oder ganz aufgehoben ist, so entsteht psychologische Reaktanz. Reaktanz ist eine motivationale Erregung mit dem Ziel, die bedrohte oder abnehmende oder gänzlich eliminierte Freiheit wiederherzustellen" (Gniech \& Dickenberger, 1987, S. 259; kursiv im Original). Daß dieses Phänomen nicht nur auf der Ebene der Handlungen, sondern auch im Bereich der Kognitionen wirksam ist, zeigen zahlreiche Untersuchungen zu diesem Thema. In einem Staat in den USA wurden phosphathaltige Waschmittel gesetzlich verboten. Die Verbraucher, die ihr bisheriges phosphathaltiges Waschmittel nicht mehr kaufen und verwenden durften, beurteilten diese Waschmittel deutlich besser als eine Vergleichsgruppe aus einem anderen Staat, in dem diese Waschmittel noch frei erhältlich waren, die die gleichen Waschmittel zu beurteilen hatte (Gniech \& Dikkenberger, 1987). Da der äußere Handlungsspielraum sehr eingeengt wurde, bewirkte die Reaktanz eine Einstellungsänderung. Der sogenannte Romeo-und-Julia-Effekt als ein Reaktanz-Phänomen (Driscoll, Davis \& Lipetz, 1972) beschreibt den Umstand, daß das Verbot einer Liebesbeziehung die Liebe der beiden Personen verstärkt, „,ie Freiheit wird durch ein Aufwerten der bedrohten Alternative (hier Partner) wiederhergestellt“ (Gniech \& Dickenberger, 1987, S. 261). Auf die Haltung der depressiven Versuchspersonen der vorliegenden Arbeit bezogen kann dies bedeuten, daß sie aufgrund des erniedrigten Aktivitätsniveaus gegen das sie sich auflehnen, ohne aber eine Änderung herbeizuführen - die innere Freiheit wiederherstellen, indem sie reaktiv von einer besonders großen Leistungssteigerung ausgehen. Im klinischen Alltag ist gerade die Ausdauer (auch im übertragenen Sinne) das Symptom bei Depressiven, gegen das sie eine besonders starke Abneigung entwickeln. Teile des im Rahmen der Depression beschriebenen Selbsthasses (Dilling, Mombour \& Schmidt, 1993) sind gerade auf diese Antriebslosigkeit bezogen, infolgederer in diesem Bereich die Wahrscheinlichkeit für ein Reaktanzphänomen deutlich erhöht war. 
Mit den Ergebnissen der zweiten Hypothese (H 2) konnte eine vergleichsweise negativere Zukunftserwartung Depressiver nicht gezeigt werden, was gegen die Grundannahmen vieler Depressionstheorien spricht (vgl. Alloy \& Ahrens, 1987, S. 366; siehe auch. S. 109). Gleichzeitig konnte auch der 'systematic bias against the self' (vgl. Alloy \& Abramson, 1988, S. 225) Depressiver nicht nachgewiesen werden. Dunning und Story (1991), die die Annahmen der 'depressive realism hypothesis' in einem 'real world setting' nicht bestätigen konnten, überlegen: „But why would nondepressed subjects exhibit greater realism in our studies and less realism in the laboratory? What is different between the two settings that would prompt these contradictory results? There are many candidates for factors to consider. One possibility is that the environment that subjects faced in these studies was more 'flexible.' Subjects had more opportunities to fulfill the 'prophecies' they had provided“ (Dunning \& Story, 1991, S. 530). Diese Ansicht entspricht in Teilen der Theorie der 'self-fulfilling prophecy' (vgl. Beck, Rush, Shaw \& Emery, 1994, S. 294), nach der allein durch die Erwartung eines Umstands die Auftretenswahrscheinlichkeit desjenigen Tatbestands erhöht wird. Experimentell haben dies in eindrucksvoller Weise Rosenthal und Jacobson (1968) gezeigt: Studenten, die als Versuchsleiter irrtümlich glaubten, ihre Versuchstiere kämen aus einem besonders lernfähigen Rattenstamm, brachten ihre Tiere auch unter hoch kontrollierten Experimentalbedingungen zu signifikant besseren Lernleistungen als Studenten, die glaubten, ihre Ratten seien weniger lernfähig. Erwartungen scheinen unbewußt die eigenen und auch fremde Handlungen in einer Weise zu steuern, daß am Ende tatsächlich das Erwartete eintritt (vgl. auch Weidenmann \& Krapp, 1986).

Die Unterscheidung von Dunning und Story (1991; s. o.) zwischen der Laborsituation und der Situation des normalen Lebens scheint jedoch für die Befunde der vorliegenden Arbeit in bezug auf die Realismushypothese nicht zutreffend. Nicht nur die Zusammenhänge zwischen der Vorhersage und dem Depressionsgrad ist nicht signifikant, auch die Zusammenhänge zwischen der Vorhersage der Leistungssteigerung und der tatsächlichen Leistungssteigerung lagen für die Kraft- und die Koordinationsbedingungen zwischen $\mathrm{r}=.01$ und $\mathrm{r}=.24$ (vgl. S. 170). Der negative Zusammenhang zwischen der Depression und der Ausdauer $(r=-.46)$ kann als Artefakt aufgrund des Reaktanzphänomens verstanden werden (s. o.), da die Depressionswerte mit der Vorhersage deutlich positiv, mit der tatsächlichen Steigerung deutlich negativ korrelierten. Eindeutig ist der Zusammenhang nur in der Schnelligkeitsbedingung, in der mit $r=.56$ die Leistungssteigerung tatsächlich eine Funktion der Vorhersage zu sein scheint und infolgedessen den einzigen eindeutigen 
'Pygmalion-Effekt ${ }^{46}$ ' (Rosenthal \& Jacobson, 1968) im Rahmen der vorliegenden Untersuchung darstellt. Hiermit hat sich auch der neuere Trend in der Goal-Setting-Forschung bestätigt: „In der zumeist experimentellen Goal-Setting-Forschung konnte u. a. nachgewiesen werden, daß hohe und konkrete Zielsetzungen zu deutlich höherer Motivation und Leistung führen als z. B. sogenannte 'Do-your-best'-Zielsetzungen. .... In den vergangenen Jahren vergrößerte sich jedoch die Anzahl der experimentellen Studien, in denen die vorhergesagten Zielsetzungseffekte im Sport nicht oder nur zum Teil nachgewiesen werden konnten“ (Kirchhoff, 1997, S. 50).

Während bei der Vorhersage der Kraftsteigerung kein Zusammenhang mit dem Grad der Depressivität festzustellen war $(r=-.02)$, zeigte sich jedoch in der tatsächlichen Steigerung (H 3), daß Depressive weniger Kraftzuwachs aufwiesen $(r=-.31)$ als Nichtdepressive. Es ist zu vermuten, daß hierfür die reduzierte Körperspannung der durch Resignation gekennzeichneten Haltung depressiver Personen verantwortlich ist; innerhalb der Depression „... leidet die Person gewöhnlich unter gedrückter Stimmung, Interessensverlust, Freudlosigkeit und einer Verminderung des Antriebs. Diese Verminderung der Energie führt zu erhöhter Ermüdbarkeit und Aktivitätseinschräkungen“ (Dilling, Mombour \& Schmidt, 1993, S. 139). Diese reduzierte Energie konnte auch innerhalb der Rehabilitationsmaßnahme für einen geringeren Aktivitätsgrad und somit für den geringeren Kraftzuwachs ursächlich sein. Unter diesem Gesichtspunkt ist auch die Betrachtung des Zusammenhangs zwischen Depression und Ausdauerzuwachs möglich, deren Korrelation $r=-.17$ betrug. In den Bereichen, die am stärksten mit den physiologischen Parametern eines allgemeinen Aktivitätsniveaus zusammenhängen, zeigte sich ein deutlicher Effekt der Depression auf die Leistungssteigerung, während in den Bereichen der Koordination keine Trends in den korrelierten Wertereihen zu erkennen sind. Schnelligkeit scheint weitgehend eine Funktion der Vorhersage zu sein ( $\mathrm{r}=.56$; s. o.). Da die Depressionswerte in keinem Zusammenhang mit der Höhe der Vorhersage stehen (H 2), ist es folgerichtig, daß der tatsächliche Leistungszuwachs nur unbedeutend mit der Schnelligkeit korrelierte $(r=.01)$. Angeleri, Angeleri, Foschi, Giaquinto und Nolfe (1993), deren Studie ,... was conducted on 180 consecutive patients affected by stroke who were hospitalized for the first time and discharged at least 1 year before the study“ (S. 1479), fanden ebenfalls einen deutlichen Zusammenhang zwischen der Depression und dem Grad der Wiederherstellung ('functional outcome'). Nach

\footnotetext{
46 „Die Schüler 'formen' sich nach dem 'Bild', das sich der Lehrer von ihnen macht; dies ist der sog.
} 'Pygmalion-Effekt'““ (Weidenmann \& Krapp, 1986, S. 408). 
telefonischer Anfrage fand ein Interview mit den ehemaligen Patienten statt. Hierbei kamen unter anderem das BDI und ADL-Skalen ${ }^{47}$ zum Einsatz. In einer „,... multiple correlation analysis .... it can be observed that weight of the Beck is far greater than that of the other variables“ (Angeleri, Angeleri, Foschi, Giaquinto \& Nolfe, 1993, S. 1480). Der geringere Rehabilitationserfolg aufgrund des Depressionsgrads bleibt folglich nicht auf die Zeit der Behandlungsmaßnahme begrenzt, sondern ist auch noch nach der Rückkehr in das soziale Umfeld wirksam. Eine Gegenposition hierzu nehmen Licoln, Jackson, Edmans, Walder, Farrow, Latham und Coombs (1990) ein, deren Untersuchung zufolge Depression kein prognostischer Faktor für das Behandlungsergebnis einer neurologischen Rehabilitation darstellte, doch sowohl nach eigener Untersuchung als auch nach Sichtung der Literatur bedürfen Depressionen nach Boné, Ladurner und Pichler (1988) „... besonderer Beachtung, da sie den Rehabilitationserfolg ungünstig beeinflussen können ... Dies ist unabhängig von der Frage, ob eine Depression im Rahmen eines Insultes reaktiv oder organisch-lokalisatorisch bedingt ist ... Daher ist diesem Aspekt der affektiven Störung als wesentliche Determinante im Langzeitverlauf Rechnung zu tragen, da durch adäquate thymoleptische Medikation oder Einsatz psychotherapeutischer Therapiekonzepte eine volle Ausschöpfung des verbliebenen Leistungspotentials möglich wird“ (S. 61; zur Frage der Ätiologie vergleiche 'Exkurs II: Depression als Folge der ZNS-Schädigung', Kap. 2.3.1.5, S. 83).

Nachdem sich die Haupthypothesen (H 1 und H 4) weitgehend bewährt haben, ergeben sich weitere Implikationen. Studien im Rahmen des Freizeitsports haben gezeigt, daß Sportler, folgen sie den Signalen des eigenen Körpers, sich häufig überbelasten (vgl. Kap. 2.2.3, S. 63). Die gleiche Beobachtung konnte auch im Rahmen einer stationären Heilbehandlung gemacht werden, innerhalb derer es mit Patienten sporttherapeutische Ziele zu verwirklichen galt: „... bei der Eingangsmessung lagen bei $80 \%$ der Versuchsgruppe und bei $90 \%$ der Kontrollgruppe die Laktatwerte, die als Indikator für die Belastungssteuerung aufgefaßt werden können, über dem Schwellenwert von 4 mmol/l“ (Pitroff, Bös \& Wydra, 1988, S. 5; vgl. auch Kap. 2.2.3, S. 63). Gemäß der vorliegenden Untersuchung handelt es sich hierbei nicht um ein einheitliches Phänomen. Vielmehr kann unter Berücksichtigung der Depression als differenzierender Faktor beobachtet werden, daß depressivere Menschen weniger in der Gefahr der Überbelastung stehen. Daraus folgt, daß im sportlichen Training die Behandlung von Sportlern anders erfolgen muß als die von Sportlern mit niedrigeren Depressionswerten. Völker (1984) überlegte im Rahmen einer Untersuchung zum Problem

\footnotetext{
47 'Activities of daily living'; z. B. Barthel-Index (Wade, 1992; siehe auch S. 134)
} 
der Belastungsintensität beim Freizeitsport: „Kann man nicht bei der weitgehenden Fremdbestimmung des beruflichen Alltags den Gesundheitssport aus diesem Gefüge ausklammern und jeden sich selbst und seinem Gefühl überlassen?“"(S. 5). Vor dem Hintergrund der in dieser Arbeit referierten Befunde (vgl. auch Kap. 2.2.3, S. 63) und Völkers eigenen Ergebnissen muß diese Frage zumindest für nichtdepressive Personen verneint werden. „Die Ergebnisse der Untersuchung zeigen, daß zwar - was die Trainingshäufigkeit pro Woche und den Trainingsumfang betrifft - eine gute Übereinstimmung mit den Empfehlungen der Literatur vorliegt. Die Belastungsintensität übersteigt jedoch bei weitem den für ein Gesundheitstraining günstigen Bereich“(Völker, 1984, S. 6 f.).

Die Beanspruchungen „,... als Destabilisierung lassen sich regelungstheoretisch als Regelgrößenauslenkungen verstehen, d. h. als Abweichungen einer zu regelnden Größe von einem vorgegebenen Wert. Solche Soll-Istwert-Diskrepanzen können sich dabei entweder durch die Einwirkung einer Störung oder durch die Veränderung des Sollwertes (der Führungsgröße) ergeben .... Art und Ausmaß psychischer Beanspruchung hängen wesentlich von selbstgesetzten Sollwerten und vor allem von der Erwartung, Wahrnehmung und Bewertung von Soll-Istwert-Diskrepanzen ab“ (Nitsch \& Hackfort, 1987, S. 35). Unter Zuhilfenahme eines kybernetischen Regelmodells gelingt die Einbindung der Schema-Theorie (vgl. S. 30). In diesem als 'Mixed Approach' bezeichneten Ansatz wurden zum ersten Mal zentrale Programmierungs- und periphere Feedbackvorgänge gemeinsam berücksichtigt, demgemäß schnelle Bewegungen ausschließlich unter der Kontrolle eines motorischen Programms stehen, langsame Bewegungen dagegen durch einen Soll-Ist-Vergleich des Feedbacks die Möglichkeit peripherer Koordination bieten (vgl. Kap. 2.1.3.2, S. 31). Das Konzept generalisierter motorischer Programme bot darüber hinaus die Lösung für Fragen, die bei den bisherigen Open- und Closed-loop-Modellen offengeblieben waren (Schmidt, 1975). „A motor program tells the muscles when to turn on, how much, and when to turn off. That is, the motor program ultimately controls force and time“ (Schmidt, 1988, S. 242; kursiv hinzugefügt). Nach der Analyse der Istwerte wird die Muskelinnervation unter Abgleichung mit den Sollwerten einen Kraftwert evozieren, der zu groß ist, da auch die nach innen gerichtete Wahrnehmung ,... schon eine gerichtete Aufmerksamkeit und ein aktives Aufnehmen ins Bewußtsein sowie eine Zuordnung zu vorhandenen Kategorien, eine subjektive Repräsentation oder Deutung“ (Westermann, 1987, S. 265; vgl. auch Kap. 2.2.2, S. 57) beinhaltet und nach den Aussagen der vierten Hypothese (H 4) der vorliegenden Arbeit die Wahrnehmung der Istwerte bei nichtdepressiven Personen geringer ausfällt als es bei einer realistischen Wahrnehmung der Fall sein müßte. „Unter neurophysiologischen Gesichtspunkten erfolgt die Wahrnehmung dabei über hierarchisch strukturierte sensomotori- 
sche Funktionskreise, die entsprechend der verschiedenen dem Menschen verfügbaren Sinne (Rezeptoren bzw. Analysatoren) in visuell-motorische, auditiv-motorische, vestibulärmotorische, propriozeptiv-motorische und taktil-motorische Funktionskreise unterteilt werden. Wahrgenommene Objekte und Sachverhalte werden über diese Funktionskreise wieder an den Menschen zurückgemeldet und bilden die Grundlage weiterer Aktionen (Handlungen)..... Die für die Steuerung und Regelung von Bewegungen erforderlichen Rückkopplungsprozesse (Rückmeldungen der Abweichungen vom vorliegenden Bewegungsplan) werden differenziert einem inneren und einem äußeren sensomotorischen Funktionsbereich zugeordnet" (Neuberg, 1982, S. 21).

Da nach den Ergebnissen der vorliegenden Studie die Wahrnehmung der Istwerte der systematischen Verzerrung folgt, stehen nichtdepressive Personen in der ständigen Gefahr einer Überbeanspruchung. Da Beanspruchung viele positive Konsequenzen beinhaltet, wie beispielsweise der im Kap. 2.3.1.4 (siehe S. 81) angesprochene depressionsvermindernde Effekt, kann das Ziel der Beanspruchungskontrolle ,... nicht in der bedingungslosen Reduktion von Beanspruchungen liegen, sondern muß sich an der Vermeidung und dem Ausgleich von Gefährdungen der physischen, psychischen und sozialen Existenz und Handlungsfähigkeit orientieren“ (Nitsch \& Hackfort, 1987, S. 39). Die hierzu notwendige Realitätsorientierung scheint aber durch die Folgerungen aus der Forschung des depressiven Realismus in Frage gestellt. Eine offenkundige Folge ist die bereits erwähnte Überbeanspruchung (s. o.; siehe auch Kap. 2.2.3, S. 63). Möglicherweise eher intuitiv als bewußt erfolgte eine diesbezügliche Prävention schon durch die Ausgabe des Mottos 'Trimming 130' als ,... die Fortsetzung der 1970 vom Deutschen Sportbund initiierten Aktion 'Trimm Dich durch Sport'...“ (Kindermann, 1984, S. 31), wobei vorausgesetzt wurde, ,... daß 30-bis 60jährige bei Erreichen einer Herzfrequenz von 130 Schlägen/min im gesundheitswirksamen Bereich trainieren, ohne sich zu überlasten“(ebd.): Auch ein Training bei 150 Schlägen/min würde im Normalfall nicht zu einer schädlichen Überbelastung führen (a. a. O., S. 33), der grobe Richtwert kann also, aufgrund dieser ausreichend großen Toleranzgrenze, für Personen mit und ohne Depressionen gelten.

Wie verhält es sich aber im leistungsorientierten Freizeitsport bzw. im Leistungsport? Dieser Frage muß unter zwei verschiedenen Aspekten nachgegangen werden, da im Freizeitbereich zumeist noch der Sportler selbst, im Leistungssport vielfach ein Trainer für das $\mathrm{Ma}$ der Beanspruchung verantwortlich ist. Für die leistungsorientierten Freizeitsportler gilt allgemein die Empfehlung, sich nur submaximal zu belasten: Dies könnte bereits, zumindest bei Personen mit einem geringen Depressionswert, der maximalen Belastung entspre- 
chen. Für Depressive, die leistungsorientierten Freizeitsport treiben, hätte dies aber eine fatale Folge: Ihre ohnehin geringe Zukunftserwartung würde in einem weiteren Bereich bestätigt. Indem sie sich mit dem Ziel optimaler Leistungssteigerungen nur submaximal belasten, erleben sie genau das, was die Theorie der Gelernten Hilflosigkeit für die Genese der Depression verantwortlich macht: Eine erfahrene und, daraus folgend, antizipierte stabile Zusammenhangslosigkeit von Verhalten und Umweltereignissen (siehe Einleitung, S. 7; vgl. auch Kap. 2.3.1.3.2, S. 74), auf diesen Fall bezogen also die partielle Zusammenhangslosigkeit von Trainingsaufwendung und Trainingserfolg. Eine Möglichkeit für dieses Problem könnte in einer differenzierteren Aufforderung an leistungsmotivierte Freizeitsportler liegen. Die Differenzierung der Zielgruppen müßte anhand eines Symptoms der Depression erfolgen, das in die Alltagssprache übersetzbar und nicht von stigmatisierendem Charakter ist. Vorstellbar wäre, eine Einteilung der Personen in 'depressiv' und 'nichtdepressiv' anhand ihrer Zukunftserwartungen vorzunehmen. Eine allgemeine Empfehlung könnte dann lauten: Für optimistische Menschen ist es eher günstig, eine nur submaximale Belastung anzustreben, während für weniger optimistische eine derartige Zurückhaltung nicht notwendig ist.

Die Frage nach der adäquaten Beanspruchung im Leistungssport unter der Anleitung eines Trainers hängt eng mit der Frage der Ethik und der der Manipulation zusammen. Im Rahmen der Trainerbezogenheit ist nicht mehr der durch den Sportler empfundene, durch die Selbstwahrnehmung vermittelte Istwert die entscheidende Größe, sondern die vom Trainer vorgegebene Größe des Sollwerts. Art und Ausmaß der Beanspruchungen hängen, wie bereits ausgeführt (s. o.), von der Soll-Istwert-Diskrepanz und damit von beiden Werten im gleichen Maße ab (Nitsch \& Hackfort, 1987, S. 35). Der vorgegebene Sollwert entspricht im Sportler selbst einer Antizipation des auf ein höheres Belastungsmaß extrapolierten, momentan erfahrbaren Istwerts, so daß durch diese Extrapolation die beim Nichtdepressiven vorhandene Verzerrung des wahrgenommenen Istwerts auch beim Sollwert Eingang findet. In bezug auf einen eher depressiven Sportler ergeben sich keine Veränderungen im Trainingsprozeß, mit Ausnahme, daß der eine oder andere 'Pessimist' nunmehr als Realist verstanden werden kann und in der Trainingssteuerung mehr Mitspracherecht erhält. Wie verhält es sich aber mit den anderen Personen, die sich eher überbeanspruchen und so von seiten des Trainers etwas geringere Sollwerte als Referenzpunkte angegeben bekommen sollten? 
Gegenstand der Ethik in diesem Sinne ist nicht nur der betriebene Sport, sondern ebenso alles Handeln, das auf die Organisation des Sports zielt ... Die Frage der Ethik nach dem Ethos des Sports umfaßt nicht nur die Frage nach dem Ethos der Sportler im Vollzug von Training und Wettkampf, sondern ebenso das Ethos der Funktionäre im Vollzug der Sportpolitik. (Herms, 1993, S. 10)

Ist es verantwortlich, Sportlern eine unrealistische Sicht zu vermitteln, um einer Überbelastung vorzubeugen? Das Ethos des Trainers, das in diesem Zusammenhang zentral wird, muß darüber entscheiden, was dieser im Umgang mit dem ihm anvertrauten Sportler verantworten kann. Der Konflikt entsteht durch die Frage, ob es im Sinne einer physiologisch ausgerichteten Trainingssteuerung ethisch unbedenklich ist, den Sportler vorsätzlich zu täuschen. Diese Frage kann weder im Rahmen der vorliegenden Arbeit noch allgemeingültig beantwortet werden: „Die Wissenschaft sagt, wie das Leben ist, die Ehik dagegen, wie es sein soll. Die Ethik entwickelt Träume oder Entwürfe vom guten oder vollkommenen Leben, hingegen die Wissenschaft den Begriff des Lebens, wie es in Wahrheit ist" (Herms, 1993, S. 103; kursiv im Original). Indem die Trainer sich mit ihrer Zielformulierung zurückhalten, können sie den Konflikt reduzieren. „In einem Überzeugungstraining lernen Sportler [dann], sich ihre Ziele selbst zu setzen und die Überzeugung aufzubauen, diese Ziele aufgrund eigener Fähigkeiten und Anstrengungen auch erreichen zu können“ (Sonnenschein, 1993, S. 184; kursiv im Original). Es ist jedoch offenkundig, daß dieses Konzept oftmals gegen die heutige Trainingsrealiät spricht.

An dieser Stelle sind einige grundlegende Gedanken zur Ethik notwendig, wobei der Schwerpunkt auf der Ethik als Theorie der Verhaltenssteuerung von personalen und sozialen Systemen liegen soll. Es handelt sich um die Frage, wie die Beziehung zwischen Trainern und Sportlern gestaltet werden kann, damit sie für beide Seiten konstruktiv ist, dem Leistungsanspruch dient und der gesellschaftlichen Einbindung gerecht wird, ohne aber gegen ethische Richtlinien zu verstoßen. Dem Begriff der Ethik liegt dabei nicht eine abstrakte Vorstellung zugrunde, sondern er folgt einem ,... in der alten Philosophie entwickelten ursprünglichen Verständnis, demzufolge 'Ethik' durchaus ein handlungsrelevantes Wissen bezeichnet: nämlich eine 'Kunstlehre', die Theorie eines regelgeleiteten Handelns“ (Herms, 1993, S. 103). Die Orientierung des Sportlers ist weitestgehend eine Orientierung an der Gesellschaft, die ihn geprägt hat, ihn beeinflußt und bestimmtes Handeln von ihm erwartet. Die Wahl der Ziele macht sich an der Vollendungsgestalt (Herms, 1993) des persönlichen, in einen größeren Sinnzusammenhang einordbaren Lebens fest. Die konkrete Zielausgestal- 
tung orientiert sich an der Vollendungsgestalt sozialer Systeme und der persönlichen Auffassung, wie diese vorstellbar sei. Der Sportler wirft den Speer, die Ausrichtung des Wurfs, der Zielpunkt und die Antriebskraft entstammen aber der - im umgebenden Publikum manifestierten - Gesellschaft. Der Trainer ist nun gleichzeitig Teil dieser Gesellschaft und durch die eingegangene soziale Beziehung mit dem Sportler Teil seines inneren Bezugssystems und nimmt somit eine besondere, verantwortungsvolle Position ein. In der Betrachtung der sportlichen Ethik wird deutlich, daß ein Mißbrauch des Sportlers nur dann verhindert werden kann, wenn sich die ethischen Vorstellungen des Sportlers mit denen seiner unmittelbaren Umgebung (Trainer, Funktionäre) decken. Trifft dies nicht zu, wird die berechnende Vermarktung des Sports systemimmanent. Herms (1993) schreibt hierzu: „Mancher hat vielleicht den Eindruck, der organisierte Sport sei zu einer ethisch qualifizierten Selbststeuerung gegenwärtig nur sehr schwer in der Lage. Es fiele mir nicht leicht, diesem Eindruck zu widersprechen. Dennoch würde ich mir wünschen, daß man es mit guten Gründen könnte“ (S. 116). Für den Konflikt des Trainers, der bislang in der Alternative zwischen ehrlicher versus manipulierter Zielvorgabe bestand, verändert sich die inhaltliche Ausgestaltung der Problematik. Der Trainer steht zwischen dem Sportler und der Gesellschaft. Für das Wohl des Sportlers und für eine humanere Gestaltung des Leistungssports wäre es wünschenswert, wenn der Trainer sich die Ethik des Sportlers zu eigen machen würde. Es liegt aber der Verdacht nahe, daß er sich oftmals dem Gesellschaftssystem, in dem er sich erst bewähren mußte, bis er an seine Position gelangt war, stärker als dem momentan zu betreuenden Sportler verpflichtet fühlt; 'Sportler kommen und gehen, die Gesellschaft aber bleibt', so die möglicherweise zugrundeliegende Maxime. Der zentrale Konflikt besteht in der Entscheidung, durch reale bis überzogene Zielvorgaben eine Leistungssteigerung anzustreben und das Risiko einer erhöhten Verletzungsgefahr sowie frühzeitiger Verschleißerscheinungen bewußt in Kauf zu nehmen. Den nichtdepressiven Sportler nicht über seine inneren, die Wahrnehmung der körperlichen Belastung verzerrenden Prozesse aufzuklären, sondern sie im Sinne der Leistungsmotivierung zu nutzen, ist das eigentliche ethische Dilemma. „Ausgehend von der Erkenntnis, daß der Mensch als ein 'homo sociologicus' immer in sozialen Beziehungen lebt und auf diese angewiesen ist, gibt es auch immer wechselseitige Beeinflussungen, bei denen der einzelne die Mitwelt manipuliert und durch sie manipuliert wird. .... Manipulation ist eine Verhaltensbeeinflussung des Menschen als Einzelwesen oder in der Gruppe zu fremdem Nutzen“ (Franke, 1987, S. 260) - im vorliegenden Fall geschieht dies zum Nutzen der Medien, der Sponsoren und nicht zuletzt der Reputation des Trainers. „Steht der Mensch noch im Mittelpunkt des Sports? .... Die realistische Frage 
kann nicht lauten, $o b$ der Mensch - gar: 'noch' - im Mittelpunkt des Sports stehe, sondern nur, wie der Mensch heute im Mittelpunkt des Sports steht. Wie gehen Menschen heute im Sport mit 'dem Menschen' - und d. h.: mit ihresgleichen - um?“ (Herms, 1993, S. 120).

Unter den Begriff der Demoralisierung (Davison \& Neale, 1988) wird ein Phänomen gefaßt, zu dessen Formenkreis geringes Selbstwertgefühl, Hilflosigkeit, Hoffnungslosigkeit, unbestimmte Zukunftsängste und allgemein gedrückte Grundstimmung zählen. Der Bewältigungsoptimismus stellt den Gegenpart dar und erfreut sich in der psychosozialen Szene besonderer Beliebtheit.

In dieser Kombination von Bewältigungs- und Unterstützungseuphorie und Überlebenskampf wirkt der zur Schau gestellte Optimismus schon beinahe verzweifelt. Die Welt wird ausgehorcht und abgeklopft, um die bislang unentdeckten Bewältigungskompetenzen und Stützpfeiler der Lebenswelten sichtbar zu machen. Doch diese Entdeckungstour nach Bewältigungsressourcen findet immer wieder ihre Grenzen, wenn sie sich nicht von einer realistischen Erschließung der gesellschaftlichen Wirklichkeit abgekoppelt hat. Ein eindrucksvolles empirisches Gegengift gegen die ideologische Beschwörung, daß in den spätkapitalistischen Gesellschaften jeder gute Chancen hätte, etwas aus seinem Leben zu machen, und aus seinen Krisen und Belastungen gestärkt und optimistisch hervorgehen könnte, liefert die neuere epidemiologische Forschung. (Davison \& Neale, 1988, S. 77)

Aus der Sicht des depressiven Realismus scheint die Beschreibung der Demoralisierung der Beschreibung der Depression zu gleichen. Die Erfahrung, in der Realität ein 'wirksames Gegengift gegen die ideologische Beschwörung' zu haben, wirkt wie die Mahnung der Depressiven zu mehr Realitätssinn. Bewirkt nun dieser Realitätssinn eine Demoralisierung? Verschiedene Ansätze haben gezeigt, daß Motivation eng mit (einem möglicherweise auch unrealistischen) Optimismus zusammenhängt (vgl. Kap. 2.3.2.4, S. 124). Nicht die Hoffnung auf unbegrenzte Bewältigungsressourcen zu vermitteln, sondern sich um die realistische Einschätzung von Bewältigungsmöglichkeiten $\mathrm{zu}$ bemühen, könnte eine mögliche Synthese darstellen. Damit wird der Blick auf die optimistisch stimmenden Aspekte des realistisch wahrgenommenen Lebens gelenkt. Ob die Motivation ausreicht, die daraus erwachsen kann, bleibt ungeklärt. Aus der realistischen Vermittlung von Bewältigungsmöglichkeiten kann auch für den Sportler diejenige Leistungsbereitschaft entstehen, die im Einklang mit den Möglichkeiten seines Organismus steht. Folgt der nichtdepressive Sport- 
ler seiner 'Bewältigungseuphorie', werden Verletzungen, chronische Überbelastungen und die Gefahr falscher Trainingsintervalle wahrscheinlich. Legt man zugrunde, daß der Realismus nicht in allen Bereichen gleichermaßen $\mathrm{zu}$ beobachten ist (vgl. Kap. 2.3.2.4.2, S. 128), wie es auch die statistische Auswertung der ersten und vierten Hypothese der vorliegenden Arbeit zeigt, kann die Empfehlung jedoch wiederum nicht eine generell geringere Belastung sein. Um Aussagen treffen zu können, für welche Bereiche welche Haltung günstig ist, ist eine weitergehende Forschung notwendig, die sich mit den differenzierenden Faktoren und den verschiedenartigen Gültigkeitsbereichen des depressiven Realismus im Sportbereich auseinandersetzt. Einer dieser Faktoren könnte in der Selbstaufmerksamkeit bestehen. Im Rahmen der Diskussion des depressiven Realismus (Kap. 2.3.2.4.1, S. 126) gewann angesichts der steigernden Wirkung der Selbstfokussierung auf den Realitätssinn (vgl. Gibbons, Ingram, Smith, Pearce \& Brehm, 1985; siehe auch S. 123) die Überlegung an Bedeutung, den depressiven Realismus mit der Theorie der selbstgerichteten Aufmerksamkeit ('self-directed attention'; vgl. Kap. 2.3.2.4.1, S. 128; vgl. auch Carver \& Scheier, 1981; Duval \& Wicklund, 1972) in Verbindung zu bringen; „... when attention is directed internally, it tends to focus on whatever aspect of the self is most salient at the time" (Gibbons \& Gaeddert, 1984, S. 162). Gibbons, Ingram, Smith, Pearce und Brehm (1985) äußerten daraufhin die Vermutung, ,... that depressed persons do tend to be chronically self-focused“ (S. 673). Wenn nun Sporttreibende die selbstgerichtete Aufmerksamkeit erhöhen, was an sich im Sinne einer adäquaten Belastungswahrnehmung naheliegt, wäre ein möglicher Weg hin zu einem nichtdepressiven Realismus beschritten. An dieser Stelle schließt sich der Kreis wieder in bezug auf die Betrachtung der Ethik des Trainers: „Innerhalb des jeweiligen Suchbereichs lenkt der Trainer die Aufmerksamkeit des Sportlers zunächst auf ein, höchstens zwei Signale, die er ... für bedeutsam hält" (Sonnenschein, 1993, S. 167). Gelingt es dem Trainer, den Sportler auf die Aspekte der Wahrnehmung aufmerksam zu machen, die eine realistische Belastungswahrnehmung bedingen, können die positiven Aspekte wirksam werden (s. o.). Die negativen, die vor allem in einer möglichen Verringerung der Leistungssteigerung bestehen, müßten dabei jedoch in Kauf genommen werden. Im Rahmen einer stationären Heilbehandlung entwickelten Pitroff, Bös und Wydra (1988) 'methodische und pädagogische Maßnahmen zur Optimierung der Traningssteuerung'. Die Konzeption des Programms beinhaltete motorische Ziele (Verbesserung der konditionellen und koordinativen Fähigkeiten), affektive Ziele (Entwicklung der Fähigkeiten zur Wahrnehmung und Kontrolle psycho-physischer Reaktionen) und kognitive Ziele (Erwerb grundlegender Kenntnisse über trainingsmethodische 
und physiologische Aspekte des Sporttreibens), deren Vermittlung in zehn Unterrichtseinheiten realisiert wurde. Nach Abschluß dieses Programms wurden die Patienten mit einer Kontrollgruppe verglichen, in der die kognitiven und affektiven Ziele während der Behandlungsmaßnahme vernachlässigt wurden: „Die Männer und Frauen der Experimentalgruppe haben ihr Basiswissen zum Ausdauertraining verbessert, und sie haben auch gelernt, die körperliche Beanspruchung während einer Ausdauerbelastung selbständig so zu steuern, daß kardiale und metabolische Überlastungserscheinungen vermieden werden und im Sinne des gesundheitsorientierten Trainingszieles ein optimaler Effekt erzielt wird“ (Pitroff, Bös \& Wydra, 1988, S. 6). Im Sinne des depressiven Realismus haben die Patienten ein Realitätstraining durchgeführt. Auch im Rahmen eines mentalen bzw. ideomotorischen Trainings (vgl. auch S. 55) kann der Sporttreibende unabhängiger von der Aufmersamkeitslenkung durch den Trainer werden und aufgrund einer selbstgesteuerten Selbstaufmerksamkeit von Außeneinflüssen frei über den Grad des eigenen Realismus selbst entscheiden.

Solche Informations-Suchstrategien können handlungsbezogen eingeübt werden, indem der Sportler entweder im Rahmen des motorischen Trainings wiederholt in die betreffende Situation, die er gezielt analysieren soll, versetzt wird und/oder indem er sich diese Situation vorstellt (mentales Training). In beiden Fällen sollte er seine Aufmerksamkeit durch entsprechende Selbstverbalisationen auf die relevanten Situationsmerkmale [auch der inneren Situation] lenken. (Sonnenschein, 1993, S. 168)

Eine allgemeine Theorie, die in der Organisation des Selbst eine Verzerrung annimmt, wurde 1980 von Anthony G. Greenwald unter dem Titel 'The Totalitarian Ego' veröffentlicht. „The most striking features of the portrait are three cognitive biases, which correspond disturbingly to thought control and propaganda devices that are considered to be defining characteristics of a totalitarian political system .... Interestingly, characteristics that seem undesirable in a political system can nonetheless serve adaptively in a personal organization of knowledge“ (Greenwald, 1980, S. 603). In der Analogie zu politischtotalitären Systemen können drei verschiedene Gruppen identifiziert werden, in die dieser 'bias' eingeordnet werden kann: Eine egozentrische, eine nützliche und eine konservative Kategorie. Die Funktion dieser Verzerrung liegt in dem Schutz des Selbst vor Gedanken, die die Integrität dieses Selbst bedrohen könnten. Depressive, bei denen der 'bias' nicht zu beobachten war, ,... suffer from a breakdown in their motivation to maintain self-esteem“ (Tennen \& Herzberger, 1987, S. 72). Wenn demzufolge die Motivation, den Selbstwert aufrecht zu erhalten, nicht mehr vorhanden ist, werden auch keine Schutzmechanismen mehr 
für dieses Selbst benötigt. Für nichtdepressive Sportler könnte dies bedeuten, daß ihre positive Sicht der eigenen Leistungsfähigkeit durch die realistische Wahrnehmung der Belastung und somit der Belastungsgrenzen gefährdet ist. Die Gegenmaßnahme (oder der Grund, weshalb sie nicht depressiv sind) könnte in dem Abwehrmechanismus der Verzerrung begründet liegen. „The knowledge system must sometimes operate with out-of-date or inaccurate information. More than compensating for these costs, presumable, are advantages: The biases help to preserve the knowledge system's organization and allow it to link effectively with behavior“" (Greenwald, 1980, S. 615).

„Woher nehmen Sportler ihren scheinbar unbegrenzten Optimismus, der... nicht allein eine Folge zurückliegender Erfahrung sein kann? Sind Behauptungen wie „Ich werde gewinnen!“ nur Teil einer motivationssteigernden Selbstinstruktion (Baumann, 1993, S. 168 ff.) oder Teile des tatsächlichen Überzeugungssystems? Nehmen Sportler sich, ihre Erfolgswahrscheinlichkeit und Leistungsentwicklung positiver wahr, als es der Realität entspricht?“ (Einleitung, Kap. 1, S. 7). Dies waren die einleitenden Fragen der vorliegenden Arbeit. Vor dem Hintergrund der bisherigen Ausführungen und der Ergebnisse der vorliegenden Untersuchung kann für den Teilbereich der Belastungswahrnehmung und der Vorhersage von Kraftsteigerungen als eine vorläufige Schlußfolgerung festgehalten werden: Die optimistische Grundhaltung entspricht nicht dem sprichwörtlichen „Pfeiffen im Wald, um sich Mut zu machen“, sondern entspringt dem tatsächlichen Überzeugungssystem. Sportler, bei denen derartige Überzeugungen in dieser Weise nicht zu beobachten sind, können als vergleichsweise depressivere Sportler verstanden werden.

In der vorliegenden Arbeit ist es weitestgehend gelungen, die Annahmen der Realismushypothese zu stützen. Sie stellt eine Replikation in einem neuen Bereich dar. Wie sich das Paradigma des depressiven Realismus jedoch insgesamt weiterentwickeln wird, ist unklar. In den letzten Jahren scheint sich das Forschungsinteresse zu reduzieren, nimmt man die Anzahl der Veröffentlichungen in der Fachliteratur als Indikator für die internationale Beachtung einer Theorie. So kann letztlich Kuhn (1962) recht behalten, nach dem ein Paradigma nicht aufgrund von konträren Ergebnissen oder schlüssigen Gegenbeweisen aufgegeben wird. In der Regel, so Kuhn (1962), stirbt es mit seinen Verfechtern aus:

Einer wissenschaftlichen Revolution geht die Krise der alten Theorie voraus. Einzelprobleme wuchern zu Anomalien aus, die sich hartnäckig jeder Lösung im Rahmen der gegebenen Theorie widersetzen .... Einige ... versuchen etwas gänzlich Neues. Damit haben sie die normale Wissenschaft verlassen; für sie beginnt die Zeit der $a u$ - 
ßerordentlichen Forschung. Daß es sich dabei um eine ganz andere Tätigkeit handelt als bei der normalen Wissenschaft, zeigt sich darin, daß die neue Theorie oder das neue Paradigma nicht allmählich aus intensiver Zusammenarbeit vieler Forscher hervorgeht. Die neue Theorie taucht vielmehr urplötzlich auf .... [Überraschend] ist die Beschreibung des Verlaufs einer wissenschaftlichen Revolution. Denn abermals scheint es sich um völlig irrationale Vorgänge zu handeln. Insbesondere kann eine solche Umwälzung nicht aufgrund von Argumenten vonstatten gehen. .... ob nun ein Anhänger des Ptolemäus mit einem Kopernikaner diskutiert oder ob Einstein gegen die Quantenphysik polemisiert - derartige Auseinandersetzungen haben stets dieselbe Form, nämlich: die Opponenten reden aneinander vorbei und gebrauchen zirkelhafte Scheinargumente. Die Gegner einer neuen Theorie sind meist Vertreter der älteren Generation von Wissenschaftlern. .... Wie kommt es dann, daß trotzdem der Widerstand gegen das Neue gebrochen wird? Die Sache ist im Grunde ebenso einfach wie brutal: Die alte Theorie und ihre Verfechter sterben aus... . (Stegmüller, 1979, S. 118 f.; kursiv im Original)

Die abschließende Betrachtung bleibt dem Paradigma des depressiven Realismus verhaftet, indem noch einmal den positiven Effekten der Realitätsverzerrung Raum gegeben wird (vgl. auch Kap. 2.3.2.4, S. 124). Jon Elster (1985) - um das Verständnis der Marxistischen Lehren bemüht - resigniert in der Suche nach einer Lösung für das von ihm als KarlMarx-Problem bezeichneten Dilemma, welches ,.... arise because some emotions that are gratifying in themselves also interfere destructively with the opportunities for gratification" (Elster, 1985, S. 376):

It suggests that there is no solution to the Karl Marx problem. Cognitive distortions just are the price we have to pay for the motivation to go on with the business of living. (Elster, 1985, S. 401) 


\section{Zusammenfassung}

Nehmen Sportler sich, ihre Erfolgswahrscheinlichkeit und ihre Leistungsentwicklung positiver wahr, als es der Realität entspricht? Unter dieser Fragestellung versucht die vorliegende Arbeit, auf dem Gebiet der Vorhersage von Leistungssteigerung und dem der durch die Belastungswahrnehmung vermittelten Selbsteinschätzung, dem Realitätssinn in Abhängigkeit vom Grad der Depressivität nachzugehen. Hierdurch gelingt der Brückenschlag von der Realismushypothese (s. u.) zu der im Sport oft beobachteten Überbelastung, die im Sinne der Realismushypothese als Selbstüberschätzung Nichtdepressiver interpretiert werden kann.

Die zentralen Annahmen der Realismushypothese besagen, daß Depressive realistischer sind, während Nichtdepressive sich und ihre Umwelt positiv verzerrt wahrnehmen. Damit widerspricht sie den klassischen Depressionstheorien, die das Phänomen der Depression mit einer negativen Weltsicht bzw. Zukunftserwartung assoziieren. Die Ursprünge der Realismushypothese liegen in den siebziger Jahren und entstammen Beobachtungen, die während einer Untersuchung im konzeptuellen Rahmen der 'Gelernten Hilflosigkeit' - welche die Genese der Depression durch die erfahrene und, daraus folgend, antizipierte stabile Zusammenhangslosigkeit zwischen Verhalten und Umweltereignissen begründet sieht - gemacht wurden: Depressive schätzten, entgegen der Vorhersagen, die sich aus der Theorie der Gelernten Hilflosigkeit ableiten lassen, die Höhe eines Zusammenhangs vergleichsweise genau ein, während er von Nichtdepressiven überschätzt wurde. Vielfältige Studien auf den Gebieten der Kontingenzschätzung, der Erwartung und Vorhersage von Ereignissen, der Attributionsmuster, der Rückmeldung und der Selbstbewertung belegten in der Folge diese Annahmen.

Die Versuche der vorliegenden Arbeit wurden mit Personen durchgeführt, für die die Steigerung der motorischen Fähigkeiten von essentieller Bedeutung ist: Bei Patienten, die aufgrund von ZNS-Schädigungen basale motorische Fähigkeiten verloren haben und diese im Rahmen einer neurologischen Rehabilitationsmaßnahme wiederzuerlangen suchten. Versuchspersonen waren 34 Patienten einer ambulanten Rehabilitationseinrichtung in Stuttgart, die während des Versuchszeitraums vom 17. Januar 1997 bis 16. Juni 1997 in dieser Einrichtung behandelt wurden. Das Durchschnittsalter der Patienten betrug 56.06 Jahre $( \pm$ 16.48), 14 Patienten waren weiblich, 20 Patienten waren männlich. Der durchschnittliche Depressionswert (BDI) lag bei $9.94( \pm 5.76)$. 
Im Gegensatz zu einer allgemeinen Validisierung in der neurologischen Rehabilitation, die sich vor allem auf die allgemeingültigen Erfordernisse des täglichen Lebens, die sogenannten 'activities of daily living' konzentrieren, erfolgte in der vorliegenden Arbeit die Operationalisierung der motorischen Leistungssteigerung als Teilprozeß innerhalb der motorischen Funktionswiederherstellung anhand der in der Sportwissenschaft üblichen Einteilung der motorischen Fähigkeit in koordinative und konditionelle Eigenschaften, wobei die konditionellen wiederum Ausdauer, Kraft und Schnelligkeit umfassen. Zu Beginn und am Ende des Rehabilitationsaufenthaltes wurden zwei Untersuchungen durchgeführt. Auf der Basis der zugrundeliegenden Hypothesen wurde zum einen erwartet, daß die Übereinstimmung der vorhergesagten Leistungssteigerung mit der tatsächlichen in Abhängigkeit vom Grad der Depressivität zunimmt, zum anderen, daß die Depressiven in der durch die Belastungswahrnehmung vermittelten Selbsteinschätzung realistischer sind.

Die Ergebnisse zeigen, daß sowohl die Einschätzungsgenauigkeit der vorhergesagten Kraftsteigerung als auch die Selbsteinschätzung mit dem Grad der Depressivität zunehmen $(r=-.36$ bzw. $r=-.40)$. Die übrigen Teilhypothesen, die die Einschätzungsgenauigkeit in bezug auf die Schnelligkeit, die Ausdauer und die Koordination zum Inhalt hatten, waren bei dem zugrundegelegten statistischen Signifikanzniveau von $\alpha=.05$ nicht signifikant. Des weiteren wurde untersucht, ob die Höhe der Vorhersage bei Depressiven geringer ist als bei nichtdepressiven Personen, und inwieweit die Depression einen Einfluß auf die Leistungssteigerung hat. Die Höhe dieser Zusammenhänge war nicht signifikant.

Die unrealistisch optimistische Grundhaltung vieler Sportler scheint ihrem tatsächlichen Überzeugungssystem zu entsprechen. Sportler, bei denen Überzeugungen in dieser Weise nicht zu beobachten sind, können als vergleichsweise depressivere Sportler verstanden werden. Die motivationalen Effekte, die aus der Verzerrung resultieren, sind vermutlich die Grundlage für die positivere Grundhaltung nichtdepressiver Personen. Sie sind möglicherweise der Preis, der für die motivationale Bewältigungsfähigkeit alltäglicher Probleme gezahlt werden muß. 


\section{Literaturverzeichnis}

Abramson, L. Y. \& Alloy, L. B. (1981). Depression, nondepression, and cognitive illusions: Reply to Schwartz. Journal of Experimental Psychology: General, 110, 436-447.

Abramson, L. Y., Alloy, L. B. \& Rosoff, R. (1981). Depression and the generation of complex hypotheses in the judgment of contingency. Behavior Research and Therapy, 19, $35-45$.

Abramson, L. Y., Metalsky, G. I. \& Alloy, L. B. (1989). Hopelessness depression: A theorybased subtype of depression. Psychological Review, 96, 358-372.

Ackermann, R. \& DeRubeis, R. J. (1991). Is depressive realism real? Clinical Psychology Review, 11, 565-584.

Adams, J. A. (1971). A closed-loop-theory of motor learning. Journal of Motor Behavior, 3, 111-150.

Adams, J. A. (1989). Lernen, Behalten und Transfer von menschlichen motorischen Fertigkeiten. Historischer Überblick und kritische Würdigung der Forschungsansätze (Schriftenreihe: Psychomotorik in Forschung und Praxis, Bd. 1). Kassel: Gesamthochschulbibliothek.

Affolter, F. (1987). Wahrnehmung, Wirklichkeit und Sprache. Neckar: VillingenSchwenningen.

Aguglia, E. \& Sapienza, S. (1986). Sport e depressione. Movimento, 2, 76-78.

Akiskal, H. S. \& McKinney, W. T. (1975). Overview of recent research in depression. Archives of General Psychiatry, 32, 285-295.

Alloy, L. B. \& Abramson, L. Y. (1979). Judgment of contingency in depressed and nondepressed students: sadder but wiser? Journal of Experimental Psychology: General, 108, 441-485.

Alloy, L. B. \& Abramson, L. Y. (1982). Learned helplessness, depression, and the illusion of control. Journal of Personality and Social Psychology, 42, 1114-1126.

Alloy, L. B. \& Abramson, L. Y. (1988). Depressive realism: Four theoretical perspectives. In L. B. Alloy (Ed.), Cognitive Processes in Depression (pp. 223-265). New York: Guilford Press.

Alloy, L. B., Abramson, L. Y. \& Kossman, D. (1985). The judgment of predictability in depressed and nondepressed college students. In F. R. Bruh \& J. B. Overmier (Eds.), Affect, conditioning, and cognition: Essay on the Determinants of Behavior (S. 229-246). Hillsdale: Erlbaum. 
Alloy, L. B., Abramson, L. Y. \& Viscusi, D. (1981). Induced mood and the illusion of control. Journal of Personality and Social Psychology. 41, 1129-1140.

Alloy, L. B. \& Ahrens, A. H. (1987). Depression and pessimism for the future: Biased use of statistically relevant information in predictions for self versus others. Journal of Personality and Social Psychology, 52, 366-378.

Alloy, L. B., Albright, J. S., Abramson, L. Y. \& Dykman, B. M. (1990). Depressive realism and nondepressive optimistic illusion: The role of the self. In R. E. Ingram (Ed.), Contemporary psychological approaches to depression: Treatment, research, and theory (pp. 71-86). New York: Plenum Press.

Alloy, L. B. \& Clements, C. M. (1992). Illusion of control: Invulnerability to negative affect and depressive symptoms after laboratory and natural stressors. Journal of Abnormal Psychology, 101, 234-245.

Alloy, L. B. \& Tabachnik, N. (1984). Assessment of covariation by humans and animals: The joint influence of prior expectations and current situational information. Psychological Review, 91, 112-149.

American Psychiatric Association (1987). Diagnostic and statistical manual of mental disorders (3rd ed., revised, DSM-III-R). Washington, DC: American Psychiatric Association.

Anderson, J. R. (1989). Kognitive Psychologie: Eine Einführung (2. Aufl.). Heidelberg: Spektrum der Wissenschaft Verlagsgesellschaft mbH \& Co.

Andres, T. (1976). Sportliche Tätigkeit als Therapie bei psychisch Kranken. Schweizer Zeitschrift für Sportmedizin: Bern, 23, 185-194.

Angeleri, F., Angeleri, V. A., Foschi, N., Giaquinto, S. \& Nolfe, G. (1993). The influence of depression, social activity, and family stress on functional outcome after stroke. Stroke, $24,1478-1483$.

Antonelli, F. (1982). Sport and depression therapy. International Journal of Sport Psychology, 13, 187-193.

Asanuma, H. \& Keller, A. (1991). Neurobiological basis of motor learning and memory. Conceptions of Neuroscience, 2, 1-30.

Banister, E. W. (1979). The perception of effort - an inductive approach. European Journal of Applied Physiology, 41, 141-150.

Basewitz, K. v., Gülick, M. v., Oetinger, R. \& Hautzinger, M. (1989). Kontingenzwahrnehmung und Depressivität: Sadder but not wiser. Eine Replikation. Schweizerische Zeitschrift für Psychologie, 48, 86-98.

Baumann, S. (1993). Psychologie im Sport. Aachen: Meyer \& Meyer Verlag. 
Beck, A. T., Rush, A. J., Shaw, B. F. \& Emery, G. (1994). Kognitive Therapie der Depressionen. Weinheim: Psychologie Verlags Union.

Benassi, V. A. \& Mahler, H. I. M. (1985). Contingency judgments by depressed college students: Sadder but not always wiser. Journal of Personality and Social Psychology, 49, 1323-1329.

Benesch, H. (1994). Neuropsychologie. In R. Ansanger \& G. Wenninger (Hrsg.), Handwörterbuch Psychologie (5. Aufl., S. 478-482). Weinheim: Beltz, Psychologie Verlags Union.

Benesch, H. (1994). Weltanschauung. In R. Ansanger \& G. Wenninger (Hrsg.), Handwörterbuch Psychologie (5. Aufl., S. 839-841). Weinheim: Beltz, Psychologie Verlags Union.

Bergholz, P. (1985). Auswirkungen von Rehabilitationsmaßnahmen auf die Kreislaufsituation nach Apoplexie. Medizinische Welt, 36, 1443-1449.

Bernstein, N. (1967). The coordination and regulation of movements. London: Pergamon.

Berry, M. J., Weyrich, A. S., Robergs, R. A., Krause, K. M. \& Ingalls, C. P. (1989). Ratings of perceived exertion in individuals with varying fitness levels during walking and running. European Journal of Applied Physiology, 58, 494-499.

Beyer, L. (1991). Zentralnervale Prozesse und sportliche Tätigkeit - Wann entdeckt (auch) die Sportmedizin unser Großhirn? Therapiewoche, 41, 1976-1984.

Blickhan, R. (1996). Motorische Steuerung bei Vertebraten. In J. Dudel, R. Menzel \& R. F. Schmidt (Hrsg.), Neurowissenschaft: Vom Molekül zur Kognition (S. 191-213). Berlin: Springer.

Bliss, T. V. P. \& Lomo, T. (1973). Long-lasting potentiation of synaptic transmission in the dentate area of the anaesthetized rabbit following stimulation of the perforant path. Journal of Physiology, 232, 331-356.

Blume, D.-D. (1981). Kennzeichnung koordinativer Fähigkeiten und Möglichkeiten ihrer Herausbildung im Trainingsprozeß. Wissenschaftliche Zeitschrift der Deutschen Hochschule für Körperkultur Leipzig, 22, 17-39.

Bobath, B. (1965). Abnormal postural reflex activity caused by brain lesions. London: Heinemann Medical Books.

Bobath, B. (1993). Die Hemiplegie Erwachsener: Befundaufnahme, Beurteilung und Behandlung (5., überarb. Aufl.). Stuttgart: Thieme.

Bohannon, R. W. \& Walsh, S. (1992). Nature, reliability, and predictive value of muscle performance measures in patients with hemiparesic following stroke. Archives of Physical Medicine and Rehabilitation, 73, 721-725. 
Boné, G., Ladurner, G. \& Pichler, M. (1988). Klinische Prognosekriterien in der Rehabilitation von Insultpatienten. Die Rehabilitation, 27, 59-62.

Borg, G. (1974). On a general scale of perceptive intensities (No. 55). Stockholm: Reports from the Institute of Applied Psychology of the University of Stockholm.

Borg, G. \& Ottoson, D. (Eds., 1986). The perception of exertion in physical work. WennerGren-Center international symposium series (Vol. 46). Basingstoke: Macmillian.

Bortz, J. (1989). Statistik für Sozialwissenschaftler (3. Aufl.). Berlin: Springer.

Bös, K. (1987). Handbuch sportmotorischer Tests. Göttingen: Verlag für Psychologie Dr. C. J. Hogrefe.

Bös K. \& Multerer, A. (1990). Diagnose und Verbesserung der Ausdauerleistungsfähigkeit im Freizeit- und Gesundheitssport. In H. J. Menzel \& Preiss, R. (Hrsg.), Forschungsgegenstand Sport (S. 56-88). Frankfurt a. M.: Festschrift für Prof. Dr. Rainer Ballreich.

Bower, G. H. \& Hilgard, E. R. (1983). Theorien des Lernens I (5. Aufl.). Stuttgart: KlettCotta.

Bradley, G. W. (1978). Self-serving biases in the attribution process: A reexamination of the fact or fiction question. Journal of Personality and Social Psychology, 36, 56-71.

Brandon, J. E. \& Loftin, J. M. (1991). Relationship of fitness to depression, state and trait anxiety, internal health locus of control, and self-control. Perceptual and Motor Skills, $73,563-568$.

Brickenkamp, R. (1975). Handbuch psychologischer und pädagogischer Tests. Göttingen: Verlag für Psychologie Dr. C. J. Hogrefe.

Brickenkamp, R. (1983). Handbuch psychologischer und pädagogischer Tests (Ergänzungsband 1). Göttingen: Verlag für Psychologie Dr. C. J. Hogrefe.

Brodmann, K. (1909). Vergleichende Lokalisationslehre der Großhirnrinde. Leipzig: J. A. Barth.

Bronner, O. (1997). Methodisch-didaktisches Konzept der Funktionellen Bewegungslehre. Krankengymnastik, 49, 578-586.

Brunnstrom, S. (1970). Movement Therapy in Hemiplegia. New York: Harper \& Row.

Bryson, S. E., Doan, B. D. \& Pasquali, P. (1984). Sadder but wiser: A failure to demonstrate that mood influences judgments of control. Canadian Journal of Behavioural Science, 16, 107-119.

Buchwald, A. M. (1977). Depressive mood and estimates of reinforcement frequency. Journal of Abnormal Psychology, 86, 443-446. 
Bueno, M. (1990). Die anaerobe Schwelle - Von der Euphorie zur Vertrauenskrise. Leistungssport, 20, 13-17.

Bund, A. (1992). Lexikon für das Kolloquium 'Motorisches Lernen'. Göttingen: Unveröffentlichtes Manuskript.

Buskies, W., Kläger, G. \& Riedel, H. (1992). Möglichkeiten zur Steuerung der Belastungsintensität für ein breitensportlich orientiertes Laufausdauertraining. Deutsche Zeitschrift für Sportmedizin, 43, 248-260.

Busse, O., Diener, H.-C., Hennerici, M. \& Sitzer, G. (1996). Bundesweite Vorsorgewoche. Gütersloh: Stiftung Deutsche Schlaganfall-Hilfe.

Bütefisch, C., Hummelsheim, H., Denzler, P. \& Mauritz, K.-H. (1995). Repetitive training of isolated movements improves the outcome of motor rehabilitation of the centrally paretic hand. Journal of the Neurological Sciences, 4559, xxx (1-11), im Druck.

Cameron, O. G. (1987). Presentations of Depression. New York: John Wiley \& Sons.

Campbell, J. D. \& Fairey, P. J. (1985). Effects of self-esteem, hypothetical explanations, and verbalization of expectancies on future performance. Journal of Personality and Social Psychology, 48, 1097-1111.

Campbell, J. D. (1986). Similarity and uniqueness: The effects of attribute type, relevance, and individual differences in self-esteem and depression. Journal of Personality and Social Psychology, 50, 281-294.

Campbell, J. D. \& Fehr, B. (1990). Self-esteem and perceptions of conveyed impressions: Is negative affectivity associated with greater realism? Journal of Personality and Social Psychology, 58, 122-133.

Cannon, W. B. \& Rosenblueth, A. (1949). The Supersensitivity of Denervated Structures. New York: Macmillan.

Carton, D. L. \& Rhodes, E. C. (1985). A critical review of the literature on rating scales for perceived exertion. Sports Medicine: Auckland, 2, 198-222.

Carver, C. S. \& Scheier, M. F. (1981). Attention and self-regulation: A control theory approach to human behavior. New York: Springer.

Coyne, J. C. \& Gotlib, I. H. (1983). The role of cognition in depression: A critical appraisal. Psychological Bulletin, 94, 472-505.

Cramon, D. Y. von, Mai, N. \& Ziegler, W. (Hrsg., 1995), Neuropsychologische Diagnostik. London: Chapman \& Hall. 
Crocker, J., Alloy, L. B. \& Kayne, N. T. (1988). Attributional style, depression, and perceptions of consensus for events. Journal of Personality and Social Psychology, 54, 840846.

Crocker, J., Tabachnik-Kayne, N. \& Alloy, L. B. (1985). Comparing the self with others in depressed and nondepressed college students: Reply to McCauley. Journal of Personality and Social Psychology, 48, 1579-1583.

Davison, G. C. \& Neale, J. M. (1996). Klinische Psychologie (4., vollst. überarbeitete u. aktual. Aufl.). Weinheim: Psychologie Verlags Union.

de Jong, R. (1987). Neurotische Depression und psychologische Therapie. Frankfurt: P. Lang.

Dean, A. (1985). Depression in multidisciplinary perspective. New York: Brunner/Mazel.

Deiber, M.-P., Passingham, R. E., Colebatch, J. G., Friston, K. J., Nixon, P. D. \& Frackowiack, R. S. J. (1991). Cortical areas and the selection of movement: a study with positron emmission tomography. Experimental Brain Research, 84, 393-402.

DeMonbreun, B. G. \& Craighead, W. E. (1977). Distortion of perception and recall of positive and neutral feedback in depression. Cognitive Therapy and Research, 1, 311-329.

Dennard, D. O. \& Hokanson, J. E. (1986). Performance on two cognitive tasks by dysphoric and nondysphoric students. Cognitive Therapy and Research, 10, 377-386.

Deschka, K. (1961). Trainingslehre und Organisationslehre des Sports. Wien: Österreichischer Bundesverlag.

Dewey, J. (1920). Reconstruction in philosophy. New York: Mentor.

Dilling, H., Mombour, W. \& Schmidt, M. H. (Hrsg., 1993). Internationale Klassifikation psychischer Störungen: ICD-10, Kapitel V (F); Klinisch-diagnostische Leitlinien (2., korr. Aufl.). Bern: Verlag Hans Huber.

Dishman, R. K., Graham, R. E., Holly, R. G. \& Tieman, J. G. (1991). Estimates of Type A behavior do not predict perceived exertion during grade exercise. Medicine and Science in Sports and Exercise, 23, 1276-1282.

Dobkin, J. A., Levine, R. L., Lagreze, H. L., Dulli, D. A., Nickles, R. J. \& Rowe, B. R. (1989). Evidence for transhemispheric diaschisis in unilateral stroke. Archive of Neurologia, 46, 1333-1336.

Dobson, K. \& Franche, R. L. (1989). A conceptual and empirical review of the depressive realism hypothesis. Canadian Journal of Behavioural Science, 21, 419-433.

Dorsch, F. (1992). Psychologisches Wörterbuch (11., ergänzte Aufl.). Bern: Verlag Hans Huber. 
Driscoll, R., Davis, K. E. \& Lipetz, M. E. (1972). Parental interference and romantic love: The Romeo and Juliet effect. Journal of Personality and Social Psychology, 24, 1-10.

Dudel, J., Menzel, R. \& Schmidt, R. F. (1996). Vorwort. In J. Dudel, R. Menzel \& R. F. Schmidt (Hrsg.), Neurowissenschaft: Vom Molekül zur Kognition (S. V). Berlin: Springer.

Dunbar, C. C., Robertson, R. J., Baun, R., Blandin, M. F., Metz, K., Burdett, R. \& Goss F. L. (1992). The validity of regulating exercise intensity by ratings of perceived exertion. Medicine and Science in Sports and Exercise, 24, 94-99.

Dunning, D. \& Story, A. L. (1991). Depression, realism, and the overconfidence effects: Are the sadder wiser when predicting future actions and events. Journal of Personality and Social Psychology, 61, 521-532.

Dunning, D., Griffin, D. W., Milojkovic, J. D. \& Ross, L. (1990). The overconfidence effect in social prediction. Journal of Personality and Social Psychology, 58, 568-581.

Duval, S. \& Wicklund, R. A. (1972). A theory of objective self-awareness. New York: Academic Press.

Dykman, B. M., Abramson, L. Y., Alloy, L. B. \& Hartlage, S. (1989). Processing of ambiguous and unambiguous feedback by depressed and nondepressed college students: Schematic biases and their implications for depressive realism. Journal of Personality and Social Psychology, 56, 431-445.

Dykman, B. M., Abramson, L. Y. \& Albright, J. S. (1991). Effects of ascending and descending patterns of success upon dysphoric and nondysphoric subjects' encoding, recall, and predictions of future success. Cognitive Therapy and Research, 15, 179-199.

Dykman, B. M., Horowitz, L. M., Abramson, L. Y. \& Usher, M. (1991). Schematic and situational determinants of depressed and nondepressed students' interpretation of feedback. Journal of Abnormal Psychology, 100, 45-55.

Ehlers, A. (o. Jahresangabe). Klinische Psychologie 1: Skript zur Vorlesung. Göttingen: Institut für Psychologie der Universität Göttingen.

Ehrenfels, Ch. v. (1890). Über Gestaltqualitäten. Vierteljahresschrift für wissenschaftliche Philosophie, 3, 249-291.

Elster, J. (1985). Sadder but wiser? Rationality and the emotions. Social Science Information, 24, 375-406.

Eston, R. G. \& Williams, J. G. (1988). Reliability of ratings of perceived effort regulation of exercise intensity. British Journal of Sports Medicine, 22, 153-155.

Evarts, E. V., Fromm, C., Kröller, J. \& Jennings, C. A. (1983). Motor cortex control to finely graded forces. Journal of Neurophysiology, 49, 1199-1215. 
Fähdrich, E., Helmchen, H. \& Linden, M. (1986). Standardized Instruments used in the assessment of depression in german-speaking countries. In N. Sartorius \& T. A. Ban (Eds.), Assessment of depression (pp. 1-8). Berlin: Springer.

Fahrenberg, J., Hampel, R. \& Selg, H. (1984). Das Freiburger Persönlichkeitsinventar (4., rev. Aufl.). Göttingen: Verlag für Psychologie Dr. C. J. Hogrefe.

Faller, A. (1995). Der Körper des Menschen (12. Auflage). Stuttgart: Thieme.

Fassheber, P., Niemeyer, H.-G. \& Kodowski, C. (1990). Methoden und Befunde der Interaktionsforschung mit dem Symlog-Konzept am Institut für Wirtschafts- und Sozialpsychologie Göttingen (18. Bericht). Göttingen: Institut für Wirschafts- und Sozialpsychologie der Georg-August-Universität Göttingen.

Fechner, G. T. (1860). Elemente der Psychophysik (Teil 1+2). Leipzig: Breitkopf und Härtel.

Feeny, D. M. \& Baron, J. C. (1986). Diaschisis. Stroke, 17, 817-830.

Fisseni, H.-J. (1990). Lehrbuch der psychologischen Diagnostik. Göttingen: Verlag für Psychologie Dr. C. J. Hogrefe.

Fleishman, E. A., Gebhardt, D. L. \& Hogan, J. C. (1984). The measurement of effort. Ergonomics, 27, 947-954.

Foerster, O. (1909). Der Lähmungstypus bei corticalen Hirnherden. Deutsche Zeitschrift für Nervenheilkunde, 37, 349-414.

Ford, C. E. \& Neale, J. M. (1985). Learned helplessness and judgments of control. Journal of Personality and Social Psychology, 49, 1330-1336.

Fors, L. (1976). Die Ausdauerscheibe für das Lauftraining. Leistungssport, Münster 6, 7678.

Franke, E. (1987). Manipulation. In H. Eberspächer (Hrsg.), Handlexikon Sportwissenschaft (S. 260-266). Reinbeck: Rowohlt Taschenbuch Verlag GmbH.

Freud, S. (1917/1946). Trauer und Melancholie. In A. Freud, E. Bibring, W. Hoffer, E. Kris \& O. Isakower (Hrsg.), Sigmund Freud: Gesammelte Werke (10. Bd., Werke aus den Jahren 1913-1917, S. 428-446). London: Imago Publishing Co.

Freund, H. J. \& Hummelsheim, H. (1985). Lesions of premotor cortex in man. Brain, 88, 697-733.

Frey, D. \& Greif, S. (1987). Sozialpsychologie: Ein Handbuch in Schlüsselbegriffen (2. Aufl.). München: Psychologie Verlags Union.

Fritze, M. (1987). Klassifikatorische Aspekte depressiver Störungen. Unveröffentl. Dissertation, Berlin. 
Fröhlich, W. D. (1993). dtv-Wörterbuch zur Psychologie (19., bearb. und erw. Aufl.). München: Deutscher Taschenbuch Verlag GmbH \& Co. KG.

Fulton, J. F. (1935). Definitions of 'motor' and 'premotor' areas. Brain, 58, 311-316.

Gadenne, V. (1994). Theorien. In T. Herrmann \& W. H. Tack (Hrsg.), Methodologische Grundlagen der Psychologie (Enzyklopädie der Psychologie, Serie Forschungsmethoden, Bd. 1; S. 295-342). Göttingen: Verlag für Psychologie Dr. C. J. Hogrefe.

Gainotti, G. (1972). Emotional behavior and hemispheric side of the lesion. Cortex, 8, 4155.

Garber, J. \& Hollon, S. D. (1980). Universal versus personal helplessness in depression: Belief in uncontrollability or incompetence? Journal of Abnormal Psychology, 89, 5666.

Gibbons, F. X. \& Gaeddert, W. P. (1984). Focus of attention and placebo utility. Journal of Experimental Social Psychology, 20, 159-176.

Gibbons, F. X., Ingram, R. E., Smith, T. W., Pearce, K. \& Brehm, S. S. (1985). Selfawareness and self-confrontation: Effects of self-focused attention on members of a clinical population. Journal of Personality and Social Psychology, 48, 662-675.

Gibson, J. J. (1979). The ecological approach to visual perception. Boston: Houghton Mifflin.

Giedke, H. (1988). Psychologisches Korrelat affektiver Störungen. In D. v. Zerrsen \& H. J. Möller (Hrsg.), Affektive Störungen: diagnostische, epidemiologische, biologische und therapeutische Aspekte (S. 131-148). Berlin: Springer.

Gniech, G. \& Dickenberger, D. (1987). Reaktanz. In D. Frey \& S. Greif (Hrsg.), Sozialpsychologie: Ein Handbuch in Schlüsselbegriffen (2. Aufl., S. 259-262). München: Psychologie Verlags Union.

Goldberg, G. (1985). Supplementary motor areastructure and function: review and hypotheses. Behavioural Brain Science, 8, 567-615.

Goldenberg, G. (1995). Praxien. In D. Y. von Cramon, N. Mai \& W. Ziegler (Hrsg.), Neuropsychologische Diagnostik (S. 259-286). London: Chapman \& Hall.

Goldstein, K. (1952). The effect of brain damage on the personality. Psychiatry, 15, 245260.

Golin, S., Terell, F. \& Johnson, B. (1977). Depression and the illusion of control. Journal of Abnormal Psychology, 86, 440-442.

Golin, S., Terrell, F., Weitz, J. \& Drost, P. L. (1979). The illusion of control among depressed patients. Journal of Abnormal Psychology, 88, 454-457. 
Golz, N. (1991). Sport, Bewegung und Spiel bei Depressionen. Sportpsychologie, 5, 22-28.

Gotlib, I. H. (1981). Self-reinforcement and recall: Differential deficits in depressed and nondepressed psychiatric inpatients. Journal of Abnormal Psychology, 90, 521-530.

Gotlib, I. H. (1983). Perception and recall of interpersonal feedback: Negative bias in depression. Cognitive Therapy and Research, 7, 399-412.

Greenwald, A. G. (1980). The totalitarian ego. American Psychologist, 35, 603-618.

Gregory, R. L. (1973). Eye and brain (2. Aufl.). New York: McGraw-Hill.

Gropler, H. \& Thieß, G. (1976). Elemente der körperlichen Leistungsfähigkeit. Theorie und Praxis der Körperkultur, 25, 127-132.

Grubitzsch, S. (1991). Testtheorie - Testpraxis. Reinbeck: Rowohlt Taschenbuch Verlag $\mathrm{GmbH}$.

Haaland, K. Y., Temkin, N., Randahl, G. \& Dikmen, S. (1994). Recovery of simple motor skills after head injury. Journal of Clinical and Experimental Neuropsychology, 16, 448-456.

Hager, W. (1987). Grundlagen einer Versuchsplanung zur Prüfung empirischer Hypothesen in der Psychologie. In G. Lüer (Hrsg.), Allgemeine experimentelle Psychologie (S. 43264). Stuttgart: Gustav Fischer Verlag.

Hallet, M. \& Khoshbin, S. (1980). A physiological mechanism of bradykinesia. Brain, 103, 301-314.

Hamilton, E. W. \& Abramson, L. Y. (1983). Cognitive patterns and major depressive disorder: A longitudinal study in a hospital setting. Journal of Abnormal Psychology, 92, 173-184.

Harre, D. (1979). Trainingslehre (8. Aufl.). Berlin: Sportverlag.

Hartje, W. \& Poek, K. (1997). Klinische Neuropsychologie (3., neubearb. Aufl.). Stuttgart: Thieme.

Hautzinger, M. \& de Jong-Meyer, R. (1994). Depression. In H. Reinecker (Hrsg.), Lehrbuch der klinischen Psychologie (2., überarb. Aufl., S. 177-218). Göttingen: Verlag für Psychologie Dr. C. J. Hogrefe.

Hautzinger, M., Bailer, M., Worall, H. \& Keller, F. (1994). Beck-Depressions-Inventar (BDI). Testhandbuch. Bern: Verlag Hans Huber.

Hegner, J. (1988). Feldtests zur Leistungsdiagnose im Ausdauerbereich. In K. Egger (Hrsg.), Sporterziehung im Wandel (S. 126-136). Bern: Schriftenreihe des Instituts für Sport und Sportwissenschaft der Universität Bern. 
Heipertz, W. (1985). Sportmedizin (7., überarb. u. erw. Aufl.). Stuttgart: Thieme.

Herms, E. (1993). Sport: Partner der Kirche und Thema der Theologie. Hannover: Lutherisches Verlagshaus.

Herrmann, M. (1992). Depressive Veränderungen nach cerebrovaskulären Insulten. Zeitschrift für Neuropsychologie, 3, 25-43.

Herrmann, M., Bartels, C., Schumacher, M. \& Wallesch, C.-W. (1995). Poststroke depression. Is there a pathoanatomic correlate for depression in the postacute stage of stroke? Stroke, 26, 850-856.

Herrmann, T. (1976). Die Psychologie und ihre Forschungsprogramme. Göttingen: Verlag für Psychologie Dr. C. J. Hogrefe.

Hesse, S. \& Mauritz, K.-H. (1994). Validisierung in der neurologischen Rehabilitation. Deutsche Zeitschrift für Sportmedizin, 45, 56-57.

Hiob 3, 20-23.26. In Thompson Studienbibel (1986), Bibeltext nach der Übersetzung Martin Luthers in der revidierten Fassung von 1984 (S. 642). Stuttgart: Hänssler-Verlag.

Hirtz, P. (1964). Zur Bewegungseigenschaft Gewandtheit. Theorie und Praxis der Körperkultur, 13, 729-735.

Hodgson, J. A., Roy, R. R., Leon, R. de, Dobkin, B. \& Edgerton, V. R. (1994). Can the mammalian lumbar spinal cord learn a motor task. Medicine and Science in Sports and Exercise, 26, 1491-1497.

Hoehn-Hyde, D., Schlottman, R. S. \& Rush, A. J. (1982). Perception of social interactions in depressed psychiatric patients. Journal of Consulting and Clinical Psychology, 50, 209-212.

Hollmann, H. (1988). Das Freiburger Persönlichkeitsinventar. Diagnostica, 34, 277-285.

Hollmann, W. \& Hettinger, T. (1980). Sportmedizin. Arbeits- und Trainingsgrundlagen (2. Aufl.). Stuttgart: Schattauer.

House, A., Dennis, M., Mogridge, L., Warlow, C., Hawton, K. \& Jones, L. (1991). Mood disorders in the year after first stroke. British Journal of Psychiatry, 158, 83-92.

Hummelsheim, H. (1996 a). Behandlungsprinzipien sensomotorischer Störungen und ihre neurophysiologischen Grundlagen. In H.-Ch. Mäurer \& H.-Ch. Diener (Hrsg.), Der Schlaganfall (S. 142-160). Stuttgart: Thieme.

Hummelsheim, H. (1996 b). Mechanismen der zentralnervösen Plastizität. In H.-Ch. Mäurer \& H.-Ch. Diener (Hrsg.), Der Schlaganfall (S. 134-138). Stuttgart: Thieme. 
Hummelsheim, H. \& Hoppe, S. (1994). Are muscular responses to magnetic brain stimulation in hemiparetic stroke patients influenced by variation in body, head and arm position? European Journal of Physical Medicine, 4, 201-204.

Hüter-Becker, A. (1997). Ein neues Denkmodell für die Physiotherapie. Krankengymnastik, $49,565-569$.

Ingram, R. E., Smith, T. W. \& Brehm, S. S. (1983). Depression and information processing: Self-schemata and the encoding of self-referent information. Journal of Personality and Social Psychology, 45, 412-420.

International Society of Sport Psychology (1992). Physical activity and psychological benefits: A position statement from the International Society of Sport Psychology. Journal of Applied Sport Psychology, 4, 94-98.

Israel, S. (1979). Sportmedizinische Positionen zu Leistungsprüfverfahren im Sport. Theorie und Praxis der Körperkultur, 28, 831-836.

Janning, M., Klingberg, S. \& Engberding, M. (1993). Bewertung eigenen und fremden Sozialverhaltens: Depressiv $=$ Realistisch? Zeitschrift für klinische Psychologie Band XXII, 39-48.

Jochheim, K.-A. (1955). Beitrag zur Beurteilung und Behandlung vegetativer Funktionsstörungen. Acta Neurovegetativa, 12, 153-166.

Johnson, D. J., Oliver, R. A. \& Terry, J. W. (1979). Regression equation for prediction of performance in the twelve minute run walk test. Journal of Sports Medicine, 19, 165170.

Kallert, T. W. \& Krebs, E. (1995). Zu Ausmaß und Verlauf depressiver Snydrome in der Frührehabilitation von Patienten mit Hirninfarkt. Zeitschrift für Neuropsychologie, 6, 314.

Kamin, L. J. (1968). Attention-like processes in classical conditioning. In R. Jones (Ed.), Miami symposium on predictability, behavior and aversive stimulation (pp. 9-32). Miami: Miami University Press.

Kammer, D. (1983). Eine Untersuchung der psychometrischen Eigenschaften des deutschen Becks-Depressions-Inventars (BDI). Diagnostica, 29, 48-60.

Kasten, E., Eder, R., Robra, B.-P. \& Sabel, A. S. (1997). Der Bedarf an ambulanter neuropsychologischer Behandlung. Zeitschrift für Neuropsychologie, 8, 72-85.

Kindermann, W. (1984). Trimming 130 und Aerobic: eine kritische Analyse. Deutsche Medizinische Wochenschrift, 10, 31-34.

Kirchhoff, O. (1997). Goal-Setting im Sport: Eine Meta-Analyse. In D. Teipel (Hrsg.), 29. Jahrestagung Arbeitsgemeinschaft für Sportpsychologie (asp). 8. 5. - 10. 5. 1997. 
Sportpsychologische Diagnostik, Prognostik, Intervention (S. 50). Jena: Institut für Sportwissenschaft Friedrich-Schiller-Universität Jena.

Klein, D. C. \& Seligman, M. E. P. (1976). Reversal of performance deficits and perceptual deficits in learned helplessness and depression. Journal of Abnormal Psychology, 85, 11-26.

Klein, M. (1987). Koordination - Koordinative Fähigkeiten. In H. Eberspächer (Hrsg.), Handlexikon Sportwissenschaft (S. 191-204). Reinbeck: Rowohlt Taschenbuch Verlag $\mathrm{GmbH}$.

Knebel, K.-P. (1985). Funktionsgymnastik. Reinbeck: Rowohlt Taschenbuch Verlag GmbH.

Knobloch, J. (1993). Psychologische Aspekte von Bewegung und Sport in der Rehabilitation. In O. Grupe (Hrsg.), Sport und Sportunterricht: Grundlagen für Studium, Ausbildung und Beruf (Einführung in die Sportpsychologie, Anwendungsfelder, Band. 3, Teil 2, S. 222-263). Schorndorf: Verlag Karl Hofmann.

Koenig, L. J., Clements, C. M. \& Alloy, L. B. (1992). Depression and the illusion of control: The role of esteem maintenance and impression management. Special Issue: The psychology of control. Canadian Journal of Behavioural Science, 24, 233-252.

Kolb, B. \& Wishaw, I. Q. (1993). Neuropsychologie. Heidelberg, Berlin, Oxford: Spektrum Akademischer Verlag.

Kowalik, D. L. \& Gotlib, I. H. (1987). Depression and marital interaction: Concordance between intent and perception of communication. Journal of Abnormal Psychology, 96, 127-134.

Krantz, S. E. (1985). When depressive cognitions reflect negative realities. Cognitive Therapy and Research, 9, 595-610.

Krause, S. (1995). Sadder but wiser - Die Selbsteinschätzung körperlich Aktiver in Abhängigkeit vom Grad der Depressivität. Göttingen: Unveröffentlichte Diplomarbeit.

Krüger, P. (1985). Aerobic aus der Sicht des Vereinsarztes. Deutsche Zeitschrift für Sportmedizin, 36, 120-123.

Kuhn, T. S. (1962). The structure of scientific revolutions. Chicago: Unversity of Chicago Press.

Kuiper, N. A. (1978). Depression and causal attributions for success and failure. Journal of Personality and Social Psychology, 36, 236-246.

Kumari, N. \& Blackburn, I. M. (1992). How specific are negative automatic thoughts to a depressed population? An exploratory study. British Journal of Medical Psychology, $65,167-176$. 
Künzle, U. (1997). Funktionelle Gangschulung bei Spastik. Krankengymnastik, 49, 588596.

Kuratorium ZNS für Unfallverletzte mit Schäden des Zentralen Nervensystems e. V. (Hrsg., 1996). ZNS-Literaturservice: Literatur zur neuropsychologischen Rehabilitation 1974 1994. Bonn: Kuratorium ZNS für Unfallverletzte mit Schäden des Zentralen Nervensystems e. V.

Kwiatkowski, G. (Red., 1987). Meyers kleines Lexikon Sport. Mannheim: Meyers Lexikonverlag.

Lamberti, G. (1993). Persönlichkeitsveränderungen nach Hirnschädigungen: Zum Stand der gegenwärtigen Diskussion. Zeitschrift für Neuropsychologie, 4, 92-103.

Lamberti, G. (1994). Persönlichkeit und Krankheitsverarbeitung nach Schlaganfall. In I. M. Ohlendorf, T. A. Pollow, W. Widdig \& D. B. Linke (Hrsg.), Sprache und Gehirn. Grundlagenforschung für die Aphasietherapie. 3. Rhein-Ruhr-Meeting in Bonn. Festschrift zum 85. Geburtstag von Anton Leischner (S. 181-194). Freiburg i. Br.: Hochschul-Verlag.

Langer, E. J. (1975). The illusion of control. Journal of Personality and Social Psychology, 32, 311-328.

Laplane, D., Talairach, J., Meininger, V., Bancaud, J. \& Bouchareine, A. (1977). Clinical consequences of corticoectomies involving the supplementary motor area in man. Journal of Neurological Sciences, 34, 301-314.

Lashley, K. S. (1929). Brain Mechanisms and Intelligence. Chicago: University of Chicago Press.

Lawrence, D. G. \& Kuypers, H. G. M. (1968). The functional organization of the motor systems in the monkey. I. The effects of bilateral pyramidal lesions. Brain, 91, 1-14.

Layne, C. (1983). Painful truths about depressives' cognitions. Journal of Clinical Psychology, 39, 848-853.

Leonhardt, G. \& Diener, H.-Ch. (1996). Akuttherapie. In H.-Ch. Mäurer \& H.-Ch. Diener (Hrsg.), Der Schlaganfall (S. 88-101). Stuttgart: Thieme.

Letzelter, M. (1987). Kondition. In H. Eberspächer (Hrsg.), Handlexikon Sportwissenschaft (S. 177-190). Reinbeck: Rowohlt Taschenbuch Verlag GmbH.

Lewinsohn, P. M., Mischel, W., Chaplin, W. \& Barton, R. (1980). Social competence and depression: The role of illusory self-perception. Journal of Abnormal Psychology, 89, 203-212. 
Licoln, N. B., Jackson, J. M., Edmans, J. A., Walder, M. F., Farrow, V. M., Latham, A. \& Coombs, K. (1990). The accuracy of predictions about progress of patients on a stroke unit. Journal of Neurology, Neurosurgery and Psychiatry, 53, 973-975.

Lieber, H.-J. (1988). Wissenschaftstheoretische Reflexion zur Sportwissenschaft. Sportwissenschaft, 18, 125-131.

Liebsch, R. (1996). Intensivkurs: Neurologie. München: Urban \& Schwarzenberg.

Lindsay, P. H. \& Norman, D. A. (1981). Einführung in die Psychologie: Informationsaufnahme und -verarbeitung beim Menschen. Berlin: Springer.

Loewenstein, D. A. \& Hokanson, J. E. (1986). The processing of social information by mildly and moderately dysphoric college students. Cognitive Therapy and Research, $10,447-460$.

Mäurer, H.-Ch. \& Diener, H.-Ch. (Hrsg., 1996). Der Schlaganfall. Stuttgart: Thieme.

Mark, D. E. \& Stevens, D. H. (1988). Patterns of personal and causal inference: Implication for the cognitive therapy of depression. In L. B. Alloy (Ed.), Cognitive Processes in Depression (pp. 344-377). New York: Guilford Press.

Markus, H. (1977). Self-schemata and processing information about the self. Journal of Personality and Social Psychology, 35, 63-78.

Marti, B. (1992). Körperbewegung und Stimmungslage. Ein epidemiologisch-präventives Kurzreview der Effekte von körperlich-sportlicher Aktivität auf psychische Verfassung und Depressionsrisiko. Deutsche Zeitschrift für Sportmedizin, 43, 336-347.

Martin, D. (1979). Grundlagen der Trainingslehre, Teil 1. Schorndorf: Verlag Karl Hofman.

Martin, D. J., Abramson, L. Y. \& Alloy, L. B. (1984). Illusion of control for self and others in depressed and nondepressed college students. Journal of Personality and Social Psychology, 46, 125-136.

Martinsen, E. W., Strand, J., Paulsson, G. \& Kaggestad, J. (1989). Physical fitness level in patients with anxiety and depressive disorders. International Journal of Sports Medicine, 10, 58-61.

Mathiowetz, V., Kashman, N., Vollan, G., Weber, K., Dowe, M. \& Rogers, S. (1985). Grip and pinch strength: Normative data for adults. Archives of Physical Medicine and Rehabilitation, 66, 69-74 .

Mattausch, W. D. (1973). Zu einigen Problemen der begrifflichen Fixierung der konditionellen und koordinativen Fähigkeiten. Theorie und Praxis der Körperkultur, 22, 849856. 
Menzel, R. (1996). Neuronale Plastizität, Lernen und Gedächtnis. In J. Dudel, R. Menzel \& R. F. Schmidt (Hrsg.), Neurowissenschaft: Vom Molekül zur Kognition (S. 485-518). Berlin: Springer.

Merten, F. \& Conradi, E. (1991). Grundlagen und Möglichkeiten sporttherapeutischer Intervention in der Schlaganfallrehabilitation. Krankengymnastik, 43, 813-818.

Mihevic, P. M. (1983). Cardiovascular fitness and the psychophysics of perceived exertion. Research Quaterly for Exercise and Sport, 54, 239-246.

Miller, D. T. \& Moretti, M. M. (1988). The causal attributions of depressives: Self-serving or self-disserving? In L. B. Alloy (Ed.), Cognitive Processes in Depression (pp. 266286). New York: Guilford Press.

Miller, D. T. \& Ross, M. (1975). Self-serving biases in the attribution of causality: Fact or fiction? Psychological Bulletin, 82, 213-225.

Milner, B. (1974). Sparing of language functions after early unilateral brain damage. In E. Eideberge \& D. G. Stein (Ed.), Functional recovery after lesions of the nervous system (pp. 213-216). Neuroscience Research Program Bulletin, 12.

Möller, H. J. (1994). Probleme der Klassifikation und Diagnostik. In H. Reinecker (Hrsg.), Lehrbuch der klinischen Psychologie (2. Aufl., S. 3-24). Göttingen: Verlag für Psychologie Dr. C. J. Hogrefe.

Monahan, T. (1988). Perceived exertion: An old exercise tool finds new applications. The Physician and Sportsmedicine: Minneapolis, 16, 174-179.

Monakow, C. von (1905). Gehirnpathologie. Wien: Holder.

Monga, T. N., Deforge, D. A., Williams, J. \& Wolfe, L. A. (1988). Cardiovascular responses to acute exercise in patients with cerebrovascular accidents. Archives of Physical Medicine and Rehabilitation, 69, 937-940.

Montada, L. (1987). Die geistige Entwicklung aus der Sicht Jean Piagets. In R. Oerter \& L. Montada (Hrsg.), Entwicklungspsychologie (2. Aufl., S. 413-462). München: Psychologie Verlags Union.

Morgan, W. P. (1993). Psychological components of effort sense. Medicine and Science in Sports and Exercise, 25, S. 1071-1077.

Mühlfriedel, B. (1979). Trainingslehre. Frankfurt a. M.: Diesterweg.

Müller, F. \& Konczack, J. (1995). Neuromotorische Kontrolle bei Funktionsstörungen der Basalganglien und des Cerebellums. Psychologische Beiträge, 37, 664-686.

Multerer, A. \& Bös, K. (1989). Felduntersuchungen zur Diagnose der aeroben Ausdauerleistungsfähigkeit - Vergleich von CONCONI-Test und Mehrstufentest (5x800m). In R. 
Daugs, K. H. Leist, H. V. Ulmer (Hrsg.), Motorikforschung aktuell. Die Elektromyographie in der Motorikforschung. Grundprobleme der Motorikforschung - interdisziplinär gesehen - Aktuelle Beiträge zur Motorik (S. 149-155). Clausthal-Zellerfeld: Deutscher Verein für Sportwissenschaften.

Munk, H. (1877). Zur Physiologie der Großhirnrinde. Berliner Klinische Wochenschrift, $14,505-506$.

Nelson, R. E. \& Craighead, W. E. (1977). Selective recall of positive and negative feedback, self-control behaviors, and depression. Journal of Abnormal Psychology, 86, 379388 .

Neuberg, E. (1982). Anregungen zur Selbstbeobachtung - Selbstkontrolle beim alpinen Skilauf. Hochschulsport, 9, 21-22.

Neuman, G. \& Schüler, K.-P. (1994). Sportmedizinische Funktionsdiagnostik (2., neubearb. Aufl.). Leipzig: Barth.

Nitsch, J. R. \& Hackfort, D. (1987). Beanspruchung. In H. Eberspächer (Hrsg.), Handlexikon Sportwissenschaft (S. 33-40). Reinbeck: Rowohlt Taschenbuch Verlag GmbH.

Noble, B. J., Borg, G. A. V., Jacobs, I., Ceci, R. \& Kaiser, P. (1983). A Category-ratio perceived exertion scale: Relationship to blood and muscle lactates and heart rate. Medicine and Science in Sports Exercise, 15, 523-528.

Nowacki, P. E. (1987). Leistungsdiagnostik, sportmedizinische. In H. Eberspächer (Hrsg.), Handlexikon Sportwissenschaft (S. 237-246). Reinbeck: Rowohlt Taschenbuch Verlag $\mathrm{GmbH}$.

Oberleit, S. (1996). Kognitive therapeutische Übungen nach Prof. Perfetti. Krankengymnastik, 48, 533-549.

Oder, W., Hufgard, J., Binder, H., Zeiler, K. \& Deecke, L. (1991). Depression, nichtsprachliche intellektuelle Beeinträchtigung und Lebensqualität nach linkshirnigem ischämischem Insult - Ergebnisse einer katamnestischen Studie. Die Rehabilitation, 30, 69-74.

Oliver, J. M., Raftery, M., Reeb, A. \& Delaney, P. (1993). Perceptions of parent-offspring relationships as functions of depression in offspring: "Affectionless control," "negative bias," and "depressive realism." Journal of Social Behavior and Personality, 8, 405-424.

Ossip-Klein, D. J., Doyne, E. J., Bowman, E. D., Osborn, K. M., McDougall-Wilson, I. B. \& Neimeyer, R. A. (1989). Effects of running or weight lifting on self-concept in clinically depressed women. Journal of Consulting and Clinical Psychology, 57, 158-161.

Pape, A. (1997). Weiterentwicklung des physiotherapeutischen Behandlungskonzeptes bei Patienten mit Rückenmarksschädigung durch das Lokomotionsprinzip von Vojta. Krankengymnastik, 49, 410-420. 
Pappata, S., Mazoyer, B., Dinh, S. T., Cambon, H., Levasseur, M. \& Baron, J. C. (1990). Effects of capsular or thalamic stroke on metabolism in the cortex and cerebellum: a positron tomography study. Stroke, 21, 519-524.

Pavlov, I. P. (1967). Lectures on conditioned reflexes. New York: International Publishers.

Penfield, W. \& Rasmussen, T. (1950). The cerebral cortex of man. New York: McMillan.

Perfetti, C. (1981). La rieducatione motoria dell' hemiplegico. Milano: Libreria scientifica già Ghedini.

Peters, H. \& Philipp, H. (1985). Zur Messung der Ausdauerleistungsfähigkeit mittels sportmotorischer Tests. Wissenschaftliche Zeitschrift der Pädagogischen Hochschule: Potsdam, 29, 494-500.

Peters, U. W. (1990). Wörterbuch der Psychiatrie und medizinischen Psychologie (4. Aufl.). München: Urban und Schwarzenberg.

Pietroff, C., Bös, K., Wydra, G. (1988). Methodische und pädagogische Maßnahmen zur Optimierung der Trainingssteuerung bei Teilnehmern an stationären Heilbehandlungen. Sporttherapie in Theorie und Praxis, 4, 3-7.

Pietromonaco, P. R. \& Markus, H. (1985). The nature of negative thoughts in depression. Journal of Personality and Social Psychology, 48, 799-807.

Poek, K. (1994). Neurologie (9., aktualisierte Aufl.). Berlin: Springer-Verlag.

Pöhlmann, R. \& Dressler, M. (1979). Gedanken zur Entwicklung konditioneller Fähigkeiten. Körpererziehung, 29, 88.

Pollock, M. L., Jackson, A. S. \& Foster, C. (1986). The use of the perception scale for exercise prescription. In G. Borg \& D. Ottoson (Eds.), The perception of exertion in physical work (pp. 161-176). Basingstoke: Macmillian.

Pöppel, E. \& Steinbüchel, N. v. (1992). Neuropsychologische Rehabilitation aus theoretischer Sicht. Zeitschrift für Medizinische Psychologie, 1, 22-30.

Pschyrembel, W. (1994). Klinisches Wörterbuch (257. Auflage). Berlin, New York: de Gruyter.

Purvis, J. W. \& Cureton, K. J. (1981). Ratings of perceived exertion at the anaerobic threshold. Ergonomics: London, 24, 295-300.

Raps, C. S., Reinhard, K. E., Peterson, C., Abramson, L. Y. \& Seligmann, M. E. P. (1982). Attributional style among depressed patients. Journal of Abnormal Psychology, 91, 102-108. 
Rathmayer, W. (1996). Motorische Steuerung bei Invertebraten. In J. Dudel, R. Menzel \& R. F. Schmidt (Hrsg.), Neurowissenschaft: Vom Molekül zur Kognition (S. 167-190). Berlin: Springer.

Rauchfleisch, U. (1994). Testpsychologie (3. Aufl.). Göttingen: Vandenhoek \& Ruprecht.

Reiner, A., Kleine, W. \& Hautzinger, M. (1990). Zur Anlage und Effektivität eines Ausdauertrainings bei leichten bis mittelschweren Depressionen. In W. Kleine \& M. Hautzinger (Hrsg.), Sport und psychisches Wohlbefinden. Beiträge zum Lehren und Lernen im Gesundheitssport (S. 70-98). Aachen: Meyer \& Meyer.

Richter, P. (1991). Zur Konstruktvalidität des Beck-Depressionsinventars (BDI) bei der Erfassung depressiver Verläufe. Regensburg: S. Roderer Verlag.

Robinson, L. M., Fitts, S. S. \& Kraft, G. H. (1990). Laterality of performance in fingertapping rate and grip strength by hemisphere of stroke and gender. Archives of Physical Medicine and Rehabilitation, 71, 695-698.

Robinson, R. G. \& Price, T. R. (1982). Post-stroke depressive disorders: a follow-up study of 103 Patients. Stroke, 13, 635-641.

Rosemeier, H. P. (1994). Medizinische Psychologie. In R. Ansanger \& G. Wenninger (Hrsg.), Handwörterbuch Psychologie (5. Aufl., S. 436-441). Weinheim: Beltz, Psychologie Verlags Union.

Rosenthal, R. \& Jacobson, L. (1968). Pygmalion in the classroom. New York: Holt, Rinehart \& Winston.

Rosner, B. S. (1970). Brain functions. Annual Review of Psychology, 21, 555-594.

Roth, K.-D. (1987). Lernen, motorisches. In H. Eberspächer (Hrsg.), Handlexikon Sportwissenschaft (S. 252-259). Reinbeck: Rowohlt Taschenbuch Verlag GmbH.

Rothwell, J. C. (1995). Watching the brain think. Current Biology, 5, 100-102.

Ruehlman L. S., West, S. G. \& Pasahow, R. J. (1985). Depression and evalutive schemata. Journal of Personality, 53, 46-92.

Sacco, W. P. \& Hokanson, J. E. (1982). Depression and self-reinforcement in a public and a private setting. Journal of Personality and Social Psychology, 42, 377-385.

Sachs, M. \& Buffone, G. W. (Eds.). Running as therapy. Nebraska: University of Nebraska Press.

Sack, H. G. (1975). Sportliche Betätigung und Persönlichkeit. Hamburg: Czwalina.

Sackheim, H. A. \& Wegner, A. Z. (1986). Attributional patterns in depression and euthymia. Archives of General Psychiatry, 43, 553-560. 
Sacks, M. H. \& Sachs, M. L. (Eds., 1981). Psychology of running. Champaign: Human Kinetics.

Salmoni, A. W. \& McIlwain, J. S. (1979). Fitt's reciprocal taping task, a measure of motor capacity? Perceptual and Motor Skills, 49, 403-413.

Scheier, M. F. \& Carver, C. S. (1985). Optimism, coping, and health: Assessment and implications of generalized outcome expectancies. Health Psychology, 4, 219-247.

Scheier, M. F. \& Carver, C. S. (1992). Effects of optimism on psychological and physical well-being: Theoretical overview and empirical update. Cognitive Therapy and Research, 16, 201-228.

Schewe, H. (1997). Bewegungslernen - Auf dem Weg zu mehr Wissen und Verständnis. Krankengymnastik, 49, 623-636.

Schildkraut, J. J. (1965). The catecholamine hypothesis of affective disorders: a review of supporting evidence. American Journal of Psychiatry, 122, 509-522.

Schlichte, C. (1990). Die Bewegungsspezifität des Hemiplegikers. Krankengymnastik, 42, 525-528.

Schlosser, G. (1995). Das pragmatische Gehirn - Wahrnehmung im Sinne der Bewegungssteuerung. Krankengymnastik, 47, 1241-1254.

Schmid, P., Berg, A., Lehmann, M., Huber, G., Jakob, E., Schwaberger, G. \& Keul, J. (1984). Feldtest zur sportartspezifischen Bestimmung der Ausdauerleistungsfähigkeit. Leistungssport: Münster, 14, 15-17.

Schmidt, R. A. (1975). A schema theory of discrete motor skill-learning. Psychological Review, 82, 225-260.

Schmidt, R. A. (1988). Motor control and learning. Champaign: Human Kinetics Publications.

Schmidt, R. F. \& Thews, G. (1985). Physiologie des Menschen (22. Aufl.). Berlin: Springer.

Schneider, G. E. (1973). Early lesions of superior colliculus: factors affecting the formation of abnormal retinal projections. Brain, Behavior and Evolution, 6, 73-105.

Schnitzler, A. \& Freund, H.-J. (1995). Elementare und komplexe Störungen motorischer Funktionen nach kortikalen Läsionen. Psychologische Beiträge, 37, 687-707.

Schober, F. (1992). Neurophysiologische Aspekte des Bewegungslernens unter besonderer Berücksichtigung des Bewegungsrhythmus. In H. Rieder (Hrsg.), Rhythmus und Bewegung (S. 57-88). Heidelberg: Institut für Sport und Sportwissenschaften.

Schürch, P. (1987). Leistungsdiagnostik. Erlangen: perimed-Fachbuch-Verlagsgesellschaft. 
Schupp, W. (1996). Wie geht es weiter? In H.-Ch. Mäurer \& H.-Ch. Diener (Hrsg.), Der Schlaganfall (S. 117-129). Stuttgart: Thieme.

Schwenkmezger, P. (1985). Welche Bedeutung kommt dem Ausdauertraining in der Depressionstherapie zu? Sportwissenschaft, 15, 117-135.

Seitelberger, F. (1968). Rehabilitation bei organischer Hirnschädigung: Grundlagen, Grenzen, Leitwege. Wiener medizinische Wochenschrift, 38/118, 738-742.

Siegel, S. J. \& Alloy, L. B. (1990). Interpersonal perceptions and consequences of depressiv-significant other relationships: A naturalistic study of college roommates. Journal of Abnormal Psychology, 99, 361-373.

Silbernagl, S. \& Despopoulus, A. (1991). Taschenatlas der Physiologie (4. Aufl.). Berlin: Springer.

Six, B. (1987). Attribution. In D. Frey \& S. Greif (Hrsg.), Sozialpsychologie: Ein Handbuch in Schlüsselbegriffen (2. Aufl., S. 122-135). München: Psychologie Verlags Union.

Skinner, B. F. (1953). Science and human behavior. New York: McMillan.

Sonnenschein, I. (1993). Psychologisches Training im Leistungssport. In H. Gabler, J. R. Nitsch \& R. Singer (Hrsg.), Einführung in die Sportpsychologie. Teil 2: Anwendungsfelder (S. 159-187). Schorndorf: Verlag Karl Hoffmann.

Staw, B. M. \& Barsade, S. G. (1993). Affect and managerial performance: A test of the sadder-but-wiser vs. happier-and-smarter hypothesis. Administrative Science Quarterly, 38, 304-331.

Steer, R. A., Beck, A. T. \& Garrison, B. (1986). Applications of the Beck Depression Inventory. In N. Sartorius \& T. A. Ban (Eds.). Assessment of depression (pp. 123-142). Berlin: Springer.

Stegmüller, W. (1979). Rationale Rekonstruktion von Wissenschaft und ihrem Wandel. Stuttgart: Reclam.

Strack, S. \& Coyne, J. C. (1983). Social confirmation of dysphoria: Shared and privat reaction to depression. Journal of Personality and Social Psychology, 44, 798-806.

Sturm, W. (1989). Theroretische Konzepte der Funktionswiederherstellung. In K. Poek (Hrsg.), Klinische Neuropsychologie (S. 359-364). Stuttgart: Thieme.

Sweeny, P. D., Anderson, K. \& Bailey, S. (1986). Attributional style in depression: A metaanalytic review. Journal of Personality and Social Psychology, 50, 974-991. 
Swerdlow, N. R. \& Kolb, G. F. (1987). Dopamine, schizophrenia, mania, and depression: Toward a unified hypothesis in cortico-striato-pallido-thalamic function. Behavioral and Brain Sciences, 10, 197-245.

Tabachnik, N., Crocker, J. \& Alloy, L. B. (1983). Depression, social comparison, and the false-consensus effect. Journal of Personality and Social Psychology, 45, 688-699.

Taylor, S. E. (1983). Adjustment to threatening events: A theory of cognitive adaptation. American Psychologist, 38, 1161-1173.

Tennen, H. \& Herzberger, S. (1987). Depression, self-esteem, and the absence o selfprotective attributional biases. Journal of Personality and Social Psychology, 52, 72-80.

Traxel, W. (1974). Grundlagen und Methoden der Psychologie (2., völlig neubearbeitete und erweiterte Auflage). Bern: Verlag Hans Huber.

Tunturipyörä Oy (o. Jahresangabe). ECB fitness ergometer E 420 - Owner's manual. Piispanristi: Tunturipyörä $\mathrm{Oy}$.

Turner, R. J., Noh, S. \& Levin, D. M. (1985). Depression across the life course: The significance of psychosocial factors among the physically disabled. In A. Dean (Ed.), Depression in multidisciplinary perspective (pp. 32-59). New York: Bruner/Mazel.

Umphred, D. (1997 a). Integration von Bewegungskontrolle und Bewegungslernen in die klinische Praxis. Krankengymnastik, 49, 637-642.

Umphred, D. (1997 b). Der Einfluß des limbischen Systems auf die Motorik. Krankengymnastik, 49, 643-649.

Vallone, R. P., Griffin, D. W., Lin, S. \& Ross, L. (1990). Overconfident prediction of future actions and outcomes by self and others. Journal of Personality and Social Psychology, $58,582-592$.

Vázquez, C. (1987). Judgment of cotingency: Cognitive biases in depressed and nondepressed subjects. Journal of Personality and Social Psychology, 52, 419-431.

Vestre, N. D. \& Caulfield, B. P. (1986). Perception of neutral personality descriptions by depressed and nondepressed subjects. Cognitive Therapy and Research, 10, 31-36.

Vogel, J.(1994). 5-Jahres-Follow-up-Studie von über 65jährigen Schlaganfallpatienten. Die Rehabilitation, 33, 155-157.

Vojta, V. (1976). Die cerebrale Bewegungsstörung im Säuglingsalter (2. Aufl.). Stuttgart: Enke.

Volk, H. (1997). Bewegung ist das Tor zum Leben. Krankengymnastik, 49, 598-599.

Völker, K. (1984). Probleme der Belastungsintensität beim Freizeitsport. Eine empirische Untersuchung. Sport und Gesundheit, 1, 5-7. 
Wade, D. T. (1992). Measurement in Neurological Rehabilitation. Oxford: Oxford Medical Publications.

Wahrig, G. (1986). Deutsches Wörterbuch. München: Mosaik Verlag.

Wall, P. D. \& Egger, M. D. (1971). Formation of new connections in adult rat brains after partial deafferentation. Nature, 232, 542-545.

Wanner, H. U. (1985). Subjektive Einstufung der Belastung bei Ausdauerleistungen. Deutsche Zeitschrift für Sportmedizin: Köln, 36, 104-112.

Ward, D. S., Jackman, J. D. \& Galiano, F. J. (1991). Exercise intensity reproduction: Children versus adults. Pediatric Exercise and Science: Champaign, 3, 209-218.

Watt, B. \& Grove, R. (1993). Perceived exertion. Antecedents and applications. Sports Medicine: Auckland, 15, 225-241.

Weber, P., Regel, H. \& Krause, A. (1995). RehaCom - Visuomotorische Koordination Helikopter, Version 2.30. Magdeburg: Hasomed GmbH.

Weber, P., Regel, H. \& Krause, A. (1996). Computergestützte Verfahren zur kognitiven Rehabilitation - REHACOM / Katalog 09/96. Magdeburg: Hasomed GmbH.

Weidenmann, B. \& Krapp, A. (Hrsg., 1986). Pädagogische Psychologie: Ein Lehrbuch. München: Psychologie Verlags Union, Urban u. Schwarzenberg.

Weiller, C. (1996). Klinik des Schlaganfalls. In H.-Ch. Mäurer \& H.-Ch. Diener (Hrsg.), Der Schlaganfall (S. 47-58). Stuttgart: Thieme.

Weiner, B. (1988). Motivationspsychologie (2. Aufl.). München: Psychologie Verlags Union.

Weinstein, N. D. (1980). Unrealistic optimism about future life events. Journal of Personality and Social Psychology, 39, 806-820.

Weinstein, N. D. (1982). Unrealistic optimism about susceptibility to health problems. Journal of Behavioral Medicine, 5, 441-460.

Werner, A. E. \& Rehm, L. P. (1975). Depressives affect: A test of behavioral hypotheses. Journal of Abnormal Psychology, 84, 221-227.

Westermann, R. (1987). Wahrnehmung: Psychophysische Skalierung und Informationsintegration. In G. Lüer (Hrsg.), Allgemeine experimentelle Psychologie (S. 265-308). Stuttgart: Gustav Fischer Verlag.

Westermann, R. (1987). Wissenschaftstheoretische Grundlagen der experimentellen Psychologie. In G. Lüer (Hrsg.), Allgemeine experimentelle Psychologie (S. 5-42). Stuttgart: Gustav Fischer Verlag. 
Weyerer S. (1992). Physical inactivity and depression in the community. Evidence from the Upper Bavarian Field Study. International Journal of Sports Medicine, 13, 492-496.

Wildmann, J. \& Krüger, A. (1986). Die Rolle endogener opioider Peptide beim Langstrekkenlauf. Deutsche Zeitschrift für Sportmedizin Köln, 37, 201-210.

Williams, J. M. \& Getty, D. (1986). Effect of levels of exercise on psychological mood states, physical fitness, and plasma beta-endorphine. Perceptual and Motor Skills, 63, 1099-1105.

Wittchen, H. U. \& Zerssen, D. v. (1988). Verläufe behandelter und unbehandelter Depressionen und Angststörungen. Berlin: Springer.

Wittling, W. (1994). Neuropsychologische Störungen. In H. Reinecker (Hrsg.), Lehrbuch der klinischen Psychologie (2., überarb. Aufl., S. 527-564). Göttingen: Verlag für Psychologie Dr. C. J. Hogrefe.

Wollny, R. (1991). Stabilität und Variabilität im motorischen Verhalten. Bielefeld: Unveröffentlichte Dissertation.

Zahnd, R. (1986). Der 12-Minuten-Lauf-Test. Magglingen, 43 (3), 24-25.

Zerfaß, R., Kretzschmar, K. \& Förstl, H. (1992). Depressive Störungen nach Hirninfarkt. Beziehungen zu Infarktlage, Hirnatrophie und kognitiven Defiziten. Der Nervenarzt, 63, 163-168.

Zerssen, D. v. (1986). Clinical Self-Rating Scales (CSRS) of the Munich Psychiatric Information System. In N. Sartorius \& T. A. Ban. Assessment of Depression. Berlin: Springer-Verlag.

Zimmermann, K. (1986). Zu spezifischen Merkmalen des Trainings koordinativer Fähigkeiten und sporttechnischer Fertigkeiten. Körpererziehung, 36, 211-215. 


\section{Anhang}

\subsection{Anhang A: Protokollblatt}

Name:

BDI-Wert:

Datum $\mathrm{t}_{1}$ :

Datum $t_{2}$ :

Die zur geschädigten Hemisphäre contralaterale Körperseite: rechts / links

\begin{tabular}{|c|c|c|c|c|c|}
\hline $\begin{array}{l}\text { Bereich } \\
\text { Test }\end{array}$ & Bedingung & der Versuche & Leistung $\mathbf{t}_{1}$ & $\begin{array}{l}\text { Vorhergesagte } \\
\text { Leistung }\end{array}$ & Leistung $\mathbf{t}_{2}$ \\
\hline & & & $\begin{array}{l}\text { Versuchen Sie, } \\
\text { so fest / } \\
\text { schnell / genau } \\
\text { wie möglich ... } \\
\text { Anweisung siehe } \\
\text { Extra-Blatt }\end{array}$ & $\begin{array}{l}\text { Sie haben jetzt ... er- } \\
\text { reicht. Wieviel, } \\
\text { glauben Sie, werden } \\
\text { Sie am Ende dieser } \\
\text { Zeit (Reha) schaf- } \\
\text { fen? }\end{array}$ & Identisch $\mathrm{zu} \mathrm{t}$ \\
\hline $\begin{array}{l}\text { Koordination: } \\
\text { Feinkoordination: }\end{array}$ & 2 min Probelauf & & & & \\
\hline $\begin{array}{l}\text { PC-Rehacom: } \\
\text { Visuomotorik }\end{array}$ & Stufe: ......... & Zeit: $\quad 180 \mathrm{sec}$ & ....... $\%$ & ......... & …..... \\
\hline Psychomotorik: & $\begin{array}{l}\text { Reinschrauben bis } \\
\text { zum leichten An- } \\
\text { schlag }\end{array}$ & $\begin{array}{l}\text { Schrauben liegen } \\
\text { auf der Seite der } \\
\text { geschädigten Hand. } \\
\text { Köpfe }\end{array}$ & & & \\
\hline $\begin{array}{l}\text { SCHRAUBENSTECK- } \\
\text { BRETT }\end{array}$ & & $\begin{array}{l}\text { nach oben, etwa } 2 \\
\text { cm Abstand }\end{array}$ & .......... sec & ............. sec & .............. sec \\
\hline $\begin{array}{l}\text { KONDITION: } \\
\text { Ausdauer: }\end{array}$ & $\begin{array}{l}\text { Steigerung alle } 2 \\
\text { min, Grenze bei HF } \\
\text { von } 130 \text { (PWC } \\
130)\end{array}$ & $\begin{array}{l}\text { RPM: ca. } 60 \\
\text { 4 Minuten locker } \\
\text { einfahren }\end{array}$ & & & \\
\hline FAHRRADERGOMETER & HF 130 & bei Stufe: ....... & Watt: ......... & Watt: ............... & Watt: ........ \\
\hline Schnelligkeit: & $\begin{array}{l}\text { So schnell wie } \\
\text { möglich, } 3 \text { x } 10 \\
\text { sec, ohne Auflegen } \\
\text { des Arms oder der } \\
\text { Hand }\end{array}$ & $\begin{array}{l}\text { Start der Messung } \\
\text { bei erster Berüh- } \\
\text { rung der Spacetaste } \\
\text { (Datei: c: Itap.doc) }\end{array}$ & $\begin{array}{l}\text { 1. Versuch:........ } \\
\text { 2. Versuch:....... } \\
\text { 3. Versuch: ........ }\end{array}$ & & $\begin{array}{l}\text { 1. Versuch:........ } \\
\text { 2. Versuch:....... } \\
\text { 3. Versuch: ........ }\end{array}$ \\
\hline TAPPING & & & $\begin{array}{r}\text { Bester Versuch: } \\
\ldots \ldots . \quad \text { x Mal }\end{array}$ & x Mal & $\begin{array}{r}\text { Bester Versuch: } \\
\ldots . . \quad \text { x Mal }\end{array}$ \\
\hline $\begin{array}{l}\text { Kraft: } \\
\qquad \& \\
\text { Belastungswahr- } \\
\text { nehmung: }\end{array}$ & $\begin{array}{l}\text { So fest wie mög- } \\
\text { lich, } 3 \text { Versuche, } \\
\text { Marke stehenlas- } \\
\text { sen, Arm hängt } \\
\text { nach unten, HD } \\
\text { seitlich in der } \\
\text { Hand, Griffweite } \\
\text { unverändert }\end{array}$ & $\begin{array}{l}\text { Vortest } \\
\text { Belastungswahr- } \\
\text { nehmung: Genau } \\
\text { mit der Hälfte der } \\
\text { subj. antizipierten } \\
\text { Maximalkraft } \\
\text { drücken }\end{array}$ & & & \\
\hline HANDDYNAMOMETER & & ...... Kg & ...... $\mathrm{Kg}$ & $\ldots \ldots$ & $\ldots \ldots$ \\
\hline
\end{tabular}

Ausfüllen: Alle mit Punkten (......) gekennzeichneten Stellen

Wenn Sie im Vergleich zu Ihrem früheren Leistungsstand, den wir als $100 \%$ bezeichenen, überlegen, wieviel Leistung sie jetzt insgesamt erbringen, wieviel Prozent wären dies? ... und auf wieviel Prozent, glauben Sie, werden Sie nach dieser Zeit (Reha) kommen?

Bemerkung: 


\subsection{Anhang B: BDI}

\section{BDI}

Name

Geburtsdatum:
Geschlecht:

Ausfälldatum:

Dieser Fragebogen enthălt 21 Gruppen yọn suşsggen. Bitse fesen Sie jede tGruppe sorgfältig dukch. Suchen Sie dann die eine Aussage in jeder Gruppe heraus, die am besten beschrejbt, wie Sie sidh in dieser Woche eiлxhlieBlich heute gefühlt haben und kreuzen Sie die dazugehörige Ziffer (0, 1, 2 oder 3) an. Falls mehrere Aussagen ęinę Gruppe gleichermaBen zutreffen, kōnnen sie auch mehrere Zifferp markieren. Lessen Sie auf jeden Fall alle Aussagen in Jeder Gruppe, bevor Sie thre Wahl trefien.

I Ich bin nicht trạtrig.

1 Ich bintraurig.

2 Ich bis die ganze Zeitt traurig und komme nicht dayon los.

3 Ith bin so traurig ader unglūck liçh, daß jch es kaym noch ertrage.

Ich sehe nicht besonders mutlos In die Zukunft.

1 lch sehe mutlos in die Zukunft.

2 Ich habe nichs, wrorauf ich mich freuen kann.

3 lch habe das Gefühl, daB die Zukunft holfnungslos ist, und daB die Situation nicht besser werden kann.

c

0 ich fühle mich nìch als versagger.

1 lch habe das Geiūhl, ötter versagt zu haben als der Durchschnitt

2 Wenn ich aư mein Leben zurūckblicke, sehe ich bloB eine Menge Fehlschläge.

3 Ich habe das Gélüh!, als Mensch ein wölliger versager zu sein.

D

- Ich kann die Dinge genauso gerię̧ęn wie früher.

1 Ich kann die Dinge nich mehr so geníeBen wíe früher.

2 Ich kann aus nicluts mehr eine ochte setrledlgumg ziehen.

3 ich bin mit allęm unzufrieden oder gelangweik.

\section{E}

0 Tth habe keine Schuldgefühle

1 Ith habe häufig schuldge luhle.

2 lch habe fast immer Schuldgefühle.

3 Ich habe immer schuldgefahle.
0 leh habe nleht das Geföhl, gestraft zu sеin.

1 Ich habe das Gefühl, viellejeht bestraft zu werden.

2 Ich erwarte, bestraft zu werden.

3 lch habe das Gefüht, bestraft zu sein.

o Ich bin nicht won mir enttäusht.

1 Ich bin von mir entrbuscht.

2 Ich firde mich fựrchterrlì̆h.

3 Ich trasse mich.

0 Ich habe nicht das Gefühl, schlechter zư seịn ạls alle anderen.

1 Ich kritisiere mich wegen melner Fehler Lnd Schwächen.

2 Ich mache mir die ganze Zeit Vorwürie wegen meiner Mänge!

3 Ich gebe mir für alles die Schuld, was schiefgeht.

Ich denke nicht daran, mir etwas anzutun.

t ich denke manchmal an Selbstmord, aber ich wüde es nicht tun.

2 Ich möchte mich am liebsten umbringen.

3 leh wourtie mith urnbringen, wenn ich die Gelegenheis thătte.

$」$

0 Ich weine nicht other fals früher

1 lch ureine jetrt mehr als früher.

2 Ich weine jetzt die ganze zeit.

3 Fräher konnte ich weinen, aber jetx kann ich es nitht męhr, obwohl ich es mochte.

Subtotal Seita 1 
K

0 Ich bin nicht reizbarer als sonst.

1 jch bin jetzt leichner werärgert oder gereizt als früher.

2 Jeh fuhle mich dauernd gerelzt.

3 Dię Dinge, dię mich frühęr geärgert haben, berühren mich nicht mehr.

$\mathbf{L}$

0 Ich babe nicht das [ibteresse an Menschen verloren.

1 lch interessiere mich jetzt weniger für Męnkchęn als früher.

2 lch habe mein interesse an anderen Menschen zum gräßten Tell verloren.

3 ich habe mein ganzes Interesse an anderen Menthen verloren:

\section{$\mathrm{M}$}

- Ich bin so entschlubfreudig vie immer.

1 ich schiebe Erstscheidungen jetzt. öfter als früher auf.

2 Es fällt mir jetzt schwerer als frühers, Entschęidungen zu treffen.

3 |ch kasn überhaspt kaine Entscheidungen mehr trefien.

N

- Ich habe niclu das Gefūhl, schlechter auszusehen a्रls früher.

1 tch mache mir Sorgen, dab ich alt oder uratratriv atssehe.

2 Ich habe das Gefohl, daB vergnderungen in męinem Aussehęn eintreten, die mich häßlich machen.

3 Icts firde micts hä̉ licts.

\section{0}

0 Iç kann so gut arbeíten wie früher.

1 Ich muB mir einen Rukx geben, bevor ich eine Tătigkeit in Angrif nehme.

2 Ich muB mich zu jeder Tätigkeit zwingen.

3 lch bin unfähig zu arbeiten.

$\mathrm{P}$

0. Ich schlarie so gut wię sonțt.

1 Ich schlafe nich mehr so gut wie frùher.

2 Içh wache 1 bis 2 Stunder früher aư als sonst, und es fāllt mir schwer, wieder einzuschlafen.

3 Ich wache mehtere Stunden fruther aluf als sorast und kann nicht mehr einschlaken.
0

lçh ermūde nichn stärket als sonst.

Ich ermüde schnellex als fruher.

Fast alles ermudet mich.

3 Ich biлk zu müde, zrn etwas zu tun.

$\mathbf{R}$

0 Meln Appetit ist richt schlechter als sonst.

1 Męin Appetit ist nicht mẹht so gut wie trüker.

2 Mein Appetit hat sehr stark nachgelassen.

3 Ich habe überhaupt keiner Appetit mehr.

$\xi$

0 Ich habe in letzter Zeit kaum abgenommen.

1 Ich habe mehr als $Z$ Kilo abgenommen.

Ich habe mehr als 5 Kilo abgenommen.

3 Ich habe mehr als 8 Kilo abgenommen.

Ich esse absichtlich weniger, um abzunepmen:

$\square$ JA $\square$ NEIN

$\uparrow$

0 lch mache mir keine größkeren Sorgen am meine Gesundheit als sonst.

1 lch mache mir Sorghen über kōrperlithe Probleme, wie Schmerzen. Magenbeschwerden oder verstopfung.

2 Ich mache mir so groBe Sorgen über gesundheitliche Probleme, dab es mix schwerfallit, an etwas anderes zw denken.

3 leh mache mir so grobe Sorgen über gesundheitliche Prableme, daB ich ari nichts anderes methr denken kann.

U

o Ich habe in letzter zelt kelne Verănderung mei. nes Interesses an ডex bemern.

1 Ich Interessiere mich weniger für sex als früher.

2 lch interessięre mich jetzt viel weriger für Sex.

3 leh habe das Interesse an Sex vollig verloren.
Subtotal seite 2

Subtotal sejte 1

Summenwert 


\subsection{Anhang C: Daten der Versuchspersonen}

\begin{tabular}{|c|c|c|c|c|c|c|c|}
\hline $\begin{array}{l}\text { Versuchsperso } \\
\text { n }\end{array}$ & Alter & Geschlecht & BDI & $\begin{array}{c}\text { Schädigungs- } \\
\text { zeitpunkt }\end{array}$ & $\begin{array}{c}\text { Geschädigte } \\
\text { Seite }\end{array}$ & Testzeitpunkt 1 & $\begin{array}{c}\text { Testzeitpunkt } \\
2\end{array}$ \\
\hline Patient 1 & 36 & $\mathrm{~m}$ & 6 & 01.08 .1996 & $\mathrm{r}$ & 07.03 .1997 & \\
\hline Patient 2 & 35 & $\mathrm{w}$ & 5 & 29.11 .1996 & $\mathrm{r}$ & 29.01 .1997 & \\
\hline Patient 3 & 28 & $\mathrm{~m}$ & 0 & 11.08 .1996 & 1 & 03.04 .1997 & 15.05 .1997 \\
\hline Patient 4 & 68 & $\mathrm{w}$ & 4 & 02.03.1997 & 1 & 10.04.1997 & 07.05 .1997 \\
\hline Patient 5 & 51 & $\mathrm{~m}$ & 7 & 01.11 .1995 & $\mathrm{r}$ & 18.04 .1997 & 28.05 .1997 \\
\hline Patient 6 & 74 & $\mathrm{~W}$ & 20 & 01.02 .1993 & $\mathrm{r}$ & 07.04 .1997 & 25.04 .1997 \\
\hline Patient 7 & 54 & $\mathrm{~W}$ & 16 & 06.12 .1996 & $\mathrm{r}$ & 06.05 .1997 & 27.05 .1997 \\
\hline Patient 8 & 47 & $\mathrm{~m}$ & 1 & 01.01 .1997 & 1 & 07.04 .1997 & 28.04 .1997 \\
\hline Patient 9 & 29 & $\mathrm{~m}$ & 9 & 31.10 .1996 & 1 & 02.06 .1997 & \\
\hline Patient 10 & 67 & $\mathrm{w}$ & 6 & 01.01 .1995 & 1 & 20.01.1997 & 06.02 .1997 \\
\hline Patient 11 & 66 & $\mathrm{~m}$ & 19 & 16.11 .1989 & 1 & 17.01 .1997 & 13.02 .1997 \\
\hline Patient 12 & 61 & $\mathrm{~m}$ & 10 & 01.03 .1995 & $\mathrm{r}$ & 21.01 .1997 & 13.02 .1997 \\
\hline Patient 13 & 77 & $\mathrm{w}$ & 7 & 01.12 .1996 & $\mathrm{r}$ & 12.01 .1997 & 10.03 .1997 \\
\hline Patient 14 & 52 & $\mathrm{~m}$ & 12 & 10.09 .1996 & 1 & 28.01 .1997 & 05.03 .1997 \\
\hline Patient 15 & 62 & $\mathrm{w}$ & 3 & 08.01 .1997 & 1 & 28.01 .1997 & 04.03 .1997 \\
\hline Patient 16 & 70 & $\mathrm{~m}$ & 11 & 15.01 .1997 & $\mathrm{r}$ & 29.01 .1997 & 25.02 .1997 \\
\hline Patient 17 & 84 & $\mathrm{~m}$ & 11 & 09.08 .1996 & $\mathrm{r}$ & 11.02 .1997 & 11.03 .1997 \\
\hline Patient 18 & 58 & $\mathrm{~W}$ & 7 & 18.11 .1996 & $\mathrm{r}$ & 10.02 .1997 & 27.02 .1997 \\
\hline Patient 19 & 61 & $\mathrm{~m}$ & 12 & 01.05 .1996 & 1 & 13.02.1997 & 14.03 .1997 \\
\hline Patient 20 & 65 & $\mathrm{w}$ & 7 & 01.06 .1994 & $\mathrm{r}$ & 10.02.1997 & 17.03 .1997 \\
\hline Patient 21 & 68 & $\mathrm{~m}$ & 5 & 12.01 .1997 & 1 & 20.02.1997 & 18.03 .1997 \\
\hline Patient 22 & 45 & $\mathrm{w}$ & 16 & 01.11 .1996 & 1 & 06.03 .1997 & 10.04 .1997 \\
\hline Patient 23 & 69 & $\mathrm{~m}$ & 12 & 01.07 .1996 & 1 & 05.03 .1997 & 03.04 .1997 \\
\hline Patient 24 & 59 & $\mathrm{~m}$ & 15 & 27.10 .1996 & $\mathrm{r}$ & 19.02 .1997 & 16.04 .1997 \\
\hline Patient 25 & 54 & $\mathrm{~m}$ & 22 & 06.04 .1996 & $\mathrm{r}$ & 19.03 .1997 & 16.04 .1997 \\
\hline Patient 26 & 47 & $\mathrm{~W}$ & 6 & 01.06 .1985 & $\mathrm{r}$ & 18.02 .1997 & 14.04 .1997 \\
\hline Patient 27 & 74 & $\mathrm{~m}$ & 15 & 01.06 .1993 & $\mathrm{r}$ & 23.04 .1997 & 28.05 .1997 \\
\hline Patient 28 & 70 & $\mathrm{~m}$ & 3 & 01.04 .1997 & $\mathrm{r}$ & 29.04.1997 & 28.05 .1997 \\
\hline Patient 29 & 68 & $\mathrm{~m}$ & 21 & 01.05 .1996 & 1 & 17.04 .1997 & 30.05 .1997 \\
\hline Patient 30 & 52 & $\mathrm{w}$ & 11 & 15.01 .1997 & 1 & 07.05 .1997 & 02.06 .1997 \\
\hline Patient 31 & 22 & $\mathrm{w}$ & 13 & 05.01 .1997 & $\mathrm{r}$ & 29.04 .1997 & 06.06 .1997 \\
\hline Patient 32 & 37 & $\mathrm{~m}$ & 3 & 01.02 .1997 & 1 & 03.06 .1997 & 11.06 .1997 \\
\hline Patient 33 & 74 & $\mathrm{~m}$ & 12 & 24.04 .1997 & $\mathrm{r}$ & 26.05 .1997 & 16.06 .1997 \\
\hline Patient 34 & 22 & $\mathrm{w}$ & 11 & 01.02 .1996 & $\mathrm{r}$ & 12.05 .1997 & 13.06 .1997 \\
\hline
\end{tabular}


8.4 Anhang D: Werte der Versuchsbedingung 'Kraft'

\begin{tabular}{|c|c|c|c|c|c|c|c|}
\hline $\begin{array}{l}\text { Versuchsperso } \\
\text { n }\end{array}$ & Alter & Geschlecht & BDI & Seite & $\begin{array}{c}\text { Testzeitpunkt } 1 \\
\text { Kilogramm }\end{array}$ & $\begin{array}{l}\text { Vorhersage } \\
\text { Kilogramm }\end{array}$ & $\begin{array}{c}\text { Testzeitpunkt } 2 \\
\text { Kilogramm }\end{array}$ \\
\hline Patient 1 & 36 & $\mathrm{~m}$ & 6 & $\mathrm{r}$ & 43 & & \\
\hline Patient 2 & 35 & $\mathrm{w}$ & 5 & $\mathrm{r}$ & 36 & & \\
\hline Patient 3 & 28 & $\mathrm{~m}$ & 0 & 1 & 22 & & \\
\hline Patient 4 & 68 & $\mathrm{w}$ & 4 & 1 & 19 & 25 & 16 \\
\hline Patient 5 & 51 & $\mathrm{~m}$ & 7 & $\mathrm{r}$ & 36 & 45 & 30 \\
\hline Patient 6 & 74 & $\mathrm{w}$ & 20 & $\mathrm{r}$ & 13 & & \\
\hline Patient 7 & 54 & $\mathrm{~W}$ & 16 & $\mathrm{r}$ & 31 & 32 & 30 \\
\hline Patient 8 & 47 & $\mathrm{~m}$ & 1 & 1 & 7 & 8 & 17 \\
\hline Patient 9 & 29 & $\mathrm{~m}$ & 9 & 1 & 42 & & \\
\hline Patient 10 & 67 & $\mathrm{w}$ & 6 & 1 & 24 & 28 & 16 \\
\hline Patient 11 & 66 & $\mathrm{~m}$ & 19 & 1 & 21 & & 18 \\
\hline Patient 12 & 61 & $\mathrm{~m}$ & 10 & $\mathrm{r}$ & 38 & 38 & 36 \\
\hline Patient 13 & 77 & $\mathrm{~W}$ & 7 & $\mathrm{r}$ & 30 & & \\
\hline Patient 14 & 52 & $\mathrm{~m}$ & 12 & 1 & 18 & 25 & 30 \\
\hline Patient 15 & 62 & $\mathrm{w}$ & 3 & 1 & 18 & 20 & 30 \\
\hline Patient 16 & 70 & $\mathrm{~m}$ & 11 & $\mathrm{r}$ & 44 & 48 & 48 \\
\hline Patient 17 & 84 & $\mathrm{~m}$ & 11 & $\mathrm{r}$ & 17 & 20 & 17 \\
\hline Patient 18 & 58 & w & 7 & $\mathrm{r}$ & 25 & 40 & 28 \\
\hline Patient 19 & 61 & $\mathrm{~m}$ & 12 & 1 & 16 & 17 & 18 \\
\hline Patient 20 & 65 & w & 7 & $\mathrm{r}$ & 18 & 20 & 22 \\
\hline Patient 21 & 68 & $\mathrm{~m}$ & 5 & 1 & 32 & 32 & 32 \\
\hline Patient 22 & 45 & $\mathrm{~W}$ & 16 & 1 & 23 & 30 & 24 \\
\hline Patient 23 & 69 & $\mathrm{~m}$ & 12 & 1 & 12 & 12 & 12 \\
\hline Patient 24 & 59 & $\mathrm{~m}$ & 15 & $\mathrm{r}$ & 36 & 36 & 42 \\
\hline Patient 25 & 54 & $\mathrm{~m}$ & 22 & $\mathrm{r}$ & 47 & & 45 \\
\hline Patient 26 & 47 & $\mathrm{w}$ & 6 & $\mathrm{r}$ & 20 & 23,5 & 18 \\
\hline Patient 27 & 74 & $\mathrm{~m}$ & 15 & $\mathrm{r}$ & 32 & 35 & 34 \\
\hline Patient 28 & 70 & $\mathrm{~m}$ & 3 & $\mathrm{r}$ & 40 & 50 & 40 \\
\hline Patient 29 & 68 & $\mathrm{~m}$ & 21 & 1 & 9 & 12 & 8 \\
\hline Patient 30 & 52 & $\mathrm{w}$ & 11 & 1 & 23 & 25 & 30 \\
\hline Patient 31 & 22 & $\mathrm{w}$ & 13 & $\mathrm{r}$ & 30 & 35 & 33 \\
\hline Patient 32 & 37 & $\mathrm{~m}$ & 3 & 1 & 47 & 50 & 44 \\
\hline Patient 33 & 74 & $\mathrm{~m}$ & 12 & $\mathrm{r}$ & 27 & 35 & 24 \\
\hline Patient 34 & 22 & $\mathrm{w}$ & 11 & $\mathrm{r}$ & 35 & 35 & 35 \\
\hline
\end{tabular}




\subsection{Anhang E: Werte der Versuchsbedingung 'Schnelligkeit'}

\begin{tabular}{|c|c|c|c|c|c|c|c|}
\hline $\begin{array}{l}\text { Versuchsperso } \\
\text { n }\end{array}$ & Alter & Geschlecht & BDI & Seite & $\begin{array}{c}\text { Testzeitpunkt } 1 \\
\text { Anschläge }\end{array}$ & $\begin{array}{l}\text { Vorhersage } \\
\text { Anschläge }\end{array}$ & $\begin{array}{c}\text { Testzeitpunkt } 2 \\
\text { Anschläge }\end{array}$ \\
\hline Patient 1 & 36 & $\mathrm{~m}$ & 6 & $\mathrm{r}$ & & & \\
\hline Patient 2 & 35 & $\mathrm{w}$ & 5 & $\mathrm{r}$ & & & \\
\hline Patient 3 & 28 & $\mathrm{~m}$ & 0 & 1 & 22 & & \\
\hline Patient 4 & 68 & $\mathrm{w}$ & 4 & 1 & 29 & 35 & 37 \\
\hline Patient 5 & 51 & $\mathrm{~m}$ & 7 & $\mathrm{r}$ & 55 & 65 & 54 \\
\hline Patient 6 & 74 & $\mathrm{~W}$ & 20 & $\mathrm{r}$ & 65 & & \\
\hline Patient 7 & 54 & $\mathrm{~W}$ & 16 & $\mathrm{r}$ & 68 & 73 & 72 \\
\hline Patient 8 & 47 & $\mathrm{~m}$ & 1 & 1 & 32 & 36 & 36 \\
\hline Patient 9 & 29 & $\mathrm{~m}$ & 9 & 1 & & & \\
\hline Patient 10 & 67 & $\mathrm{~W}$ & 6 & 1 & 26 & 30 & 31 \\
\hline Patient 11 & 66 & $\mathrm{~m}$ & 19 & 1 & 49 & 55 & 43 \\
\hline Patient 12 & 61 & $\mathrm{~m}$ & 10 & $\mathrm{r}$ & 66 & 70 & 57 \\
\hline Patient 13 & 77 & $\mathrm{w}$ & 7 & $\mathrm{r}$ & 67 & & \\
\hline Patient 14 & 52 & $\mathrm{~m}$ & 12 & 1 & 48 & 58 & 59 \\
\hline Patient 15 & 62 & $\mathrm{~W}$ & 3 & 1 & 43 & 50 & 45 \\
\hline Patient 16 & 70 & $\mathrm{~m}$ & 11 & $\mathrm{r}$ & 66 & 70 & 72 \\
\hline Patient 17 & 84 & $\mathrm{~m}$ & 11 & $\mathrm{r}$ & 27 & 35 & 28 \\
\hline Patient 18 & 58 & $\mathrm{~W}$ & 7 & $\mathrm{r}$ & 58 & 70 & 64 \\
\hline Patient 19 & 61 & $\mathrm{~m}$ & 12 & 1 & 32 & 35 & 35 \\
\hline Patient 20 & 65 & $\mathrm{~W}$ & 7 & $\mathrm{r}$ & 41 & 50 & 59 \\
\hline Patient 21 & 68 & $\mathrm{~m}$ & 5 & 1 & 60 & & 71 \\
\hline Patient 22 & 45 & $\mathrm{w}$ & 16 & 1 & 34 & 75 & 54 \\
\hline Patient 23 & 69 & $\mathrm{~m}$ & 12 & 1 & 42 & 60 & 45 \\
\hline Patient 24 & 59 & $\mathrm{~m}$ & 15 & $\mathrm{r}$ & 71 & 71 & 65 \\
\hline Patient 25 & 54 & $\mathrm{~m}$ & 22 & $\mathrm{r}$ & 95 & 100 & 90 \\
\hline Patient 26 & 47 & $\mathrm{w}$ & 6 & $\mathrm{r}$ & 23 & 23 & 26 \\
\hline Patient 27 & 74 & $\mathrm{~m}$ & 15 & $\mathrm{r}$ & 24 & 35 & 50 \\
\hline Patient 28 & 70 & $\mathrm{~m}$ & 3 & $\mathrm{r}$ & 57 & 80 & 61 \\
\hline Patient 29 & 68 & $\mathrm{~m}$ & 21 & 1 & 24 & 35 & 26 \\
\hline Patient 30 & 52 & $\mathrm{~W}$ & 11 & 1 & 60 & 70 & 62 \\
\hline Patient 31 & 22 & $\mathrm{w}$ & 13 & $\mathrm{r}$ & 77 & 77 & 80 \\
\hline Patient 32 & 37 & $\mathrm{~m}$ & 3 & 1 & 60 & 60 & 61 \\
\hline Patient 33 & 74 & $\mathrm{~m}$ & 12 & $\mathrm{r}$ & 59 & 70 & 57 \\
\hline Patient 34 & 22 & $\mathrm{~W}$ & 11 & $\mathrm{r}$ & 59 & 70 & 62 \\
\hline
\end{tabular}


8.6 Anhang F: Werte der Versuchsbedingung 'Ausdauer'

\begin{tabular}{|c|c|c|c|c|c|c|c|}
\hline $\begin{array}{l}\text { Versuchsperso } \\
\text { n }\end{array}$ & Alter & Geschlecht & BDI & Seite & $\begin{array}{c}\text { Testzeitpunkt } 1 \\
\text { Watt }\end{array}$ & $\begin{array}{c}\text { Vorhersage } \\
\text { Watt }\end{array}$ & $\begin{array}{c}\text { Testzeitpunkt } 2 \\
\text { Watt }\end{array}$ \\
\hline Patient 1 & 36 & $\mathrm{~m}$ & 6 & $\mathrm{r}$ & & & \\
\hline Patient 2 & 35 & $\mathrm{w}$ & 5 & $\mathrm{r}$ & & & \\
\hline Patient 3 & 28 & $\mathrm{~m}$ & 0 & 1 & & & \\
\hline Patient 4 & 68 & $\mathrm{w}$ & 4 & 1 & 85 & 90 & 100 \\
\hline Patient 5 & 51 & $\mathrm{~m}$ & 7 & $\mathrm{r}$ & 70 & 80 & 100 \\
\hline Patient 6 & 74 & $\mathrm{w}$ & 20 & $\mathrm{r}$ & & & \\
\hline Patient 7 & 54 & $\mathrm{w}$ & 16 & $\mathrm{r}$ & 90 & 92 & 110 \\
\hline Patient 8 & 47 & $\mathrm{~m}$ & 1 & 1 & 95 & 97 & 105 \\
\hline Patient 9 & 29 & $\mathrm{~m}$ & 9 & 1 & & & \\
\hline Patient 10 & 67 & $\mathrm{~W}$ & 6 & 1 & & & \\
\hline Patient 11 & 66 & $\mathrm{~m}$ & 19 & 1 & 90 & 100 & 90 \\
\hline Patient 12 & 61 & $\mathrm{~m}$ & 10 & $\mathrm{r}$ & & & \\
\hline Patient 13 & 77 & $\mathrm{~W}$ & 7 & $\mathrm{r}$ & & & \\
\hline Patient 14 & 52 & $\mathrm{~m}$ & 12 & 1 & 85 & & \\
\hline Patient 15 & 62 & $\mathrm{w}$ & 3 & 1 & 55 & 55 & 65 \\
\hline Patient 16 & 70 & $\mathrm{~m}$ & 11 & $\mathrm{r}$ & & & \\
\hline Patient 17 & 84 & $\mathrm{~m}$ & 11 & $\mathrm{r}$ & & & \\
\hline Patient 18 & 58 & $\mathrm{~W}$ & 7 & $\mathrm{r}$ & & & \\
\hline Patient 19 & 61 & $\mathrm{~m}$ & 12 & 1 & 70 & & 85 \\
\hline Patient 20 & 65 & $\mathrm{~W}$ & 7 & $\mathrm{r}$ & & & \\
\hline Patient 21 & 68 & $\mathrm{~m}$ & 5 & 1 & & & \\
\hline Patient 22 & 45 & $\mathrm{w}$ & 16 & 1 & 80 & & 85 \\
\hline Patient 23 & 69 & $\mathrm{~m}$ & 12 & 1 & & & \\
\hline Patient 24 & 59 & $\mathrm{~m}$ & 15 & $\mathrm{r}$ & & & \\
\hline Patient 25 & 54 & $\mathrm{~m}$ & 22 & $\mathrm{r}$ & 55 & 65 & 50 \\
\hline Patient 26 & 47 & $\mathrm{~W}$ & 6 & $\mathrm{r}$ & & & \\
\hline Patient 27 & 74 & $\mathrm{~m}$ & 15 & $\mathrm{r}$ & & & \\
\hline Patient 28 & 70 & $\mathrm{~m}$ & 3 & $\mathrm{r}$ & 80 & 85 & 85 \\
\hline Patient 29 & 68 & $\mathrm{~m}$ & 21 & 1 & 40 & 55 & 55 \\
\hline Patient 30 & 52 & $\mathrm{w}$ & 11 & 1 & 30 & 35 & 30 \\
\hline Patient 31 & 22 & $\mathrm{~W}$ & 13 & $\mathrm{r}$ & 75 & 90 & 60 \\
\hline Patient 32 & 37 & $\mathrm{~m}$ & 3 & 1 & 95 & 100 & 110 \\
\hline Patient 33 & 74 & $\mathrm{~m}$ & 12 & $\mathrm{r}$ & 85 & 85 & 90 \\
\hline Patient 34 & 22 & $\mathrm{~W}$ & 11 & $\mathrm{r}$ & 60 & 80 & 75 \\
\hline
\end{tabular}




\subsection{Anhang G: Werte der Versuchsbedingung 'Koordination I'}

\begin{tabular}{|c|c|c|c|c|c|c|c|}
\hline $\begin{array}{l}\text { Versuchsperso } \\
\text { n }\end{array}$ & Alter & Geschlecht & BDI & Seite & $\begin{array}{c}\text { Testzeitpunkt } 1 \\
\text { Fehlerzeit } \%\end{array}$ & $\begin{array}{l}\text { Vorhersage } \\
\text { Fehlerzeit \% }\end{array}$ & $\begin{array}{c}\text { Testzeitpunkt } 2 \\
\text { Fehlerzeit } \%\end{array}$ \\
\hline Patient 1 & 36 & $\mathrm{~m}$ & 6 & $\mathrm{r}$ & & & \\
\hline Patient 2 & 35 & $\mathrm{w}$ & 5 & $\mathrm{r}$ & & & \\
\hline Patient 3 & 28 & $\mathrm{~m}$ & 0 & 1 & 58 & & \\
\hline Patient 4 & 68 & $\mathrm{w}$ & 4 & 1 & 18 & 5 & 3 \\
\hline Patient 5 & 51 & $\mathrm{~m}$ & 7 & $\mathrm{r}$ & 11 & 5 & 6 \\
\hline Patient 6 & 74 & $\mathrm{~W}$ & 20 & $\mathrm{r}$ & & & \\
\hline Patient 7 & 54 & $\mathrm{w}$ & 16 & $\mathrm{r}$ & 33 & 20 & 11 \\
\hline Patient 8 & 47 & $\mathrm{~m}$ & 1 & 1 & 49 & 35 & 51 \\
\hline Patient 9 & 29 & $\mathrm{~m}$ & 9 & 1 & & & \\
\hline Patient 10 & 67 & $\mathrm{~W}$ & 6 & 1 & 5 & 2 & 2 \\
\hline Patient 11 & 66 & $\mathrm{~m}$ & 19 & 1 & 2 & 1 & 1 \\
\hline Patient 12 & 61 & $\mathrm{~m}$ & 10 & $\mathrm{r}$ & 11 & 8 & 7 \\
\hline Patient 13 & 77 & $\mathrm{~W}$ & 7 & $\mathrm{r}$ & & & \\
\hline Patient 14 & 52 & $\mathrm{~m}$ & 12 & 1 & 13 & 3 & 10 \\
\hline Patient 15 & 62 & $\mathrm{~W}$ & 3 & 1 & 20 & 10 & 5 \\
\hline Patient 16 & 70 & $\mathrm{~m}$ & 11 & $\mathrm{r}$ & 4 & 4 & 5 \\
\hline Patient 17 & 84 & $\mathrm{~m}$ & 11 & $\mathrm{r}$ & 23 & 12 & 13 \\
\hline Patient 18 & 58 & $\mathrm{w}$ & 7 & $\mathrm{r}$ & 70 & 40 & 30 \\
\hline Patient 19 & 61 & $\mathrm{~m}$ & 12 & 1 & 13 & 8 & 6 \\
\hline Patient 20 & 65 & $\mathrm{~W}$ & 7 & $\mathrm{r}$ & 73 & 60 & 51 \\
\hline Patient 21 & 68 & $\mathrm{~m}$ & 5 & 1 & 17 & 7 & 4 \\
\hline Patient 22 & 45 & $\mathrm{w}$ & 16 & 1 & 24 & 24 & 9 \\
\hline Patient 23 & 69 & $\mathrm{~m}$ & 12 & 1 & 22 & 22 & 3 \\
\hline Patient 24 & 59 & $\mathrm{~m}$ & 15 & $\mathrm{r}$ & 11 & 6 & 7 \\
\hline Patient 25 & 54 & $\mathrm{~m}$ & 22 & $\mathrm{r}$ & 5 & 2,5 & 2 \\
\hline Patient 26 & 47 & $\mathrm{w}$ & 6 & $\mathrm{r}$ & & & \\
\hline Patient 27 & 74 & $\mathrm{~m}$ & 15 & $\mathrm{r}$ & 21 & 10 & 18 \\
\hline Patient 28 & 70 & $\mathrm{~m}$ & 3 & $\mathrm{r}$ & 52 & 10 & 26 \\
\hline Patient 29 & 68 & $\mathrm{~m}$ & 21 & 1 & & & \\
\hline Patient 30 & 52 & $\mathrm{w}$ & 11 & 1 & 22 & 15 & 20 \\
\hline Patient 31 & 22 & $\mathrm{w}$ & 13 & $\mathrm{r}$ & 5 & 3 & 2 \\
\hline Patient 32 & 37 & $\mathrm{~m}$ & 3 & 1 & 23 & 15 & 14 \\
\hline Patient 33 & 74 & $\mathrm{~m}$ & 12 & $\mathrm{r}$ & 9 & 5 & 6 \\
\hline Patient 34 & 22 & $\mathrm{~W}$ & 11 & $\mathrm{r}$ & 1 & & \\
\hline
\end{tabular}


8.8 Anhang H: Werte der Versuchsbedingung 'Koordination II'

\begin{tabular}{|c|c|c|c|c|c|c|c|}
\hline $\begin{array}{l}\text { Versuchsperso } \\
\text { n }\end{array}$ & Alter & Geschlecht & BDI & Seite & $\begin{array}{c}\text { Testzeitpunkt } 1 \\
\text { Sekunden }\end{array}$ & $\begin{array}{c}\text { Vorhersage } \\
\text { Sekunden }\end{array}$ & $\begin{array}{c}\text { Testzeitpunkt } 2 \\
\text { Sekunden }\end{array}$ \\
\hline Patient 1 & 36 & $\mathrm{~m}$ & 6 & $\mathrm{r}$ & & & \\
\hline Patient 2 & 35 & $\mathrm{w}$ & 5 & $\mathrm{r}$ & & & \\
\hline Patient 3 & 28 & $\mathrm{~m}$ & 0 & 1 & & & \\
\hline Patient 4 & 68 & $\mathrm{w}$ & 4 & 1 & 45 & 40 & 51 \\
\hline Patient 5 & 51 & $\mathrm{~m}$ & 7 & $\mathrm{r}$ & 59 & 45 & 66 \\
\hline Patient 6 & 74 & $\mathrm{w}$ & 20 & $\mathrm{r}$ & 20 & & \\
\hline Patient 7 & 54 & $\mathrm{~W}$ & 16 & $\mathrm{r}$ & 24 & 20 & 23 \\
\hline Patient 8 & 47 & $\mathrm{~m}$ & 1 & 1 & 144 & 90 & 133 \\
\hline Patient 9 & 29 & $\mathrm{~m}$ & 9 & 1 & & & \\
\hline Patient 10 & 67 & $\mathrm{w}$ & 6 & 1 & 166 & 120 & 117 \\
\hline Patient 11 & 66 & $\mathrm{~m}$ & 19 & 1 & 92 & 70 & 89 \\
\hline Patient 12 & 61 & $\mathrm{~m}$ & 10 & $\mathrm{r}$ & 42 & 38 & 44 \\
\hline Patient 13 & 77 & $\mathrm{~W}$ & 7 & $\mathrm{r}$ & 24 & & \\
\hline Patient 14 & 52 & $\mathrm{~m}$ & 12 & 1 & 33 & 29 & 33 \\
\hline Patient 15 & 62 & $\mathrm{w}$ & 3 & 1 & 54 & 40 & 55 \\
\hline Patient 16 & 70 & $\mathrm{~m}$ & 11 & $\mathrm{r}$ & 42 & 42 & 27 \\
\hline Patient 17 & 84 & $\mathrm{~m}$ & 11 & $\mathrm{r}$ & 105 & 70 & 103 \\
\hline Patient 18 & 58 & $\mathrm{w}$ & 7 & $\mathrm{r}$ & 28 & 20 & 22 \\
\hline Patient 19 & 61 & $\mathrm{~m}$ & 12 & 1 & 122 & 90 & 96 \\
\hline Patient 20 & 65 & w & 7 & $\mathrm{r}$ & 86 & 86 & 67 \\
\hline Patient 21 & 68 & $\mathrm{~m}$ & 5 & 1 & 88 & 30 & 50 \\
\hline Patient 22 & 45 & $\mathrm{~W}$ & 16 & 1 & 38 & 25 & 34 \\
\hline Patient 23 & 69 & $\mathrm{~m}$ & 12 & 1 & 181 & 120 & 97 \\
\hline Patient 24 & 59 & $\mathrm{~m}$ & 15 & $\mathrm{r}$ & 72 & 40 & 78 \\
\hline Patient 25 & 54 & $\mathrm{~m}$ & 22 & $\mathrm{r}$ & 20 & 17 & 23 \\
\hline Patient 26 & 47 & $\mathrm{w}$ & 6 & $\mathrm{r}$ & & & \\
\hline Patient 27 & 74 & $\mathrm{~m}$ & 15 & $\mathrm{r}$ & & & \\
\hline Patient 28 & 70 & $\mathrm{~m}$ & 3 & $\mathrm{r}$ & 31 & 20 & 31 \\
\hline Patient 29 & 68 & $\mathrm{~m}$ & 21 & 1 & & & \\
\hline Patient 30 & 52 & $\mathrm{w}$ & 11 & 1 & 65 & 60 & 33 \\
\hline Patient 31 & 22 & $\mathrm{~W}$ & 13 & $\mathrm{r}$ & 19,6 & 19,6 & 19 \\
\hline Patient 32 & 37 & $\mathrm{~m}$ & 3 & 1 & 20,1 & 20,1 & 18,8 \\
\hline Patient 33 & 74 & $\mathrm{~m}$ & 12 & $\mathrm{r}$ & 23 & 23 & 24,7 \\
\hline Patient 34 & 22 & $\mathrm{w}$ & 11 & $\mathrm{r}$ & 26,8 & 26,8 & 21,7 \\
\hline
\end{tabular}


8.9 Anhang I: Werte der Versuchsbedingung 'Belastungswahrnehmung'

\begin{tabular}{|c|c|c|c|c|c|c|c|}
\hline $\begin{array}{l}\text { Versuchsperso } \\
\text { n }\end{array}$ & Alter & Geschlecht & BDI & $\begin{array}{c}\text { Schädigungs- } \\
\text { zeitpunkt }\end{array}$ & Seite & $\begin{array}{l}\text { Halbe Kraft } \\
\text { Kilogramm }\end{array}$ & $\begin{array}{c}\text { Maximalkraft } \\
\text { Kilogramm }\end{array}$ \\
\hline Patient 1 & 36 & $\mathrm{~m}$ & 6 & 01.08 .1996 & $\mathrm{r}$ & 36 & 43 \\
\hline Patient 2 & 35 & $\mathrm{w}$ & 5 & 29.11 .1996 & $\mathrm{r}$ & 17 & 36 \\
\hline Patient 3 & 28 & $\mathrm{~m}$ & 0 & 11.08 .1996 & 1 & 22 & 22 \\
\hline Patient 4 & 68 & $\mathrm{w}$ & 4 & 02.03 .1997 & 1 & 14 & 19 \\
\hline Patient 5 & 51 & $\mathrm{~m}$ & 7 & 01.11 .1995 & $\mathrm{r}$ & 5 & 36 \\
\hline Patient 6 & 74 & $\mathrm{w}$ & 20 & 01.02 .1993 & $\mathrm{r}$ & 5 & 13 \\
\hline Patient 7 & 54 & $\mathrm{w}$ & 16 & 06.12 .1996 & $\mathrm{r}$ & 14 & 31 \\
\hline Patient 8 & 47 & $\mathrm{~m}$ & 1 & 01.01 .1997 & 1 & 5 & 7 \\
\hline Patient 9 & 29 & $\mathrm{~m}$ & 9 & 31.10 .1996 & 1 & 12 & 42 \\
\hline Patient 10 & 67 & $\mathrm{w}$ & 6 & 01.01 .1995 & 1 & 14 & 24 \\
\hline Patient 11 & 66 & $\mathrm{~m}$ & 19 & 16.11 .1989 & 1 & 7 & 21 \\
\hline Patient 12 & 61 & $\mathrm{~m}$ & 10 & 01.03 .1995 & $\mathrm{r}$ & 27 & 38 \\
\hline Patient 13 & 77 & $\mathrm{w}$ & 7 & 01.12 .1996 & $\mathrm{r}$ & 25 & 30 \\
\hline Patient 14 & 52 & $\mathrm{~m}$ & 12 & 10.09 .1996 & 1 & 12 & 18 \\
\hline Patient 15 & 62 & $\mathrm{w}$ & 3 & 08.01 .1997 & 1 & 5 & 18 \\
\hline Patient 16 & 70 & $\mathrm{~m}$ & 11 & 15.01 .1997 & $\mathrm{r}$ & & 44 \\
\hline Patient 17 & 84 & $\mathrm{~m}$ & 11 & 09.08 .1996 & $\mathrm{r}$ & 6 & 17 \\
\hline Patient 18 & 58 & $\mathrm{w}$ & 7 & 18.11 .1996 & $\mathrm{r}$ & 20 & 25 \\
\hline Patient 19 & 61 & $\mathrm{~m}$ & 12 & 01.05 .1996 & 1 & 11 & 16 \\
\hline Patient 20 & 65 & $\mathrm{w}$ & 7 & 01.06 .1994 & $\mathrm{r}$ & 14 & 18 \\
\hline Patient 21 & 68 & $\mathrm{~m}$ & 5 & 12.01 .1997 & 1 & 16 & 32 \\
\hline Patient 22 & 45 & $\mathrm{w}$ & 16 & 01.11 .1996 & 1 & 10 & 23 \\
\hline Patient 23 & 69 & $\mathrm{~m}$ & 12 & 01.07 .1996 & 1 & 4 & 12 \\
\hline Patient 24 & 59 & $\mathrm{~m}$ & 15 & 27.10 .1996 & $\mathrm{r}$ & 15 & 36 \\
\hline Patient 25 & 54 & $\mathrm{~m}$ & 22 & 06.04 .1996 & $\mathrm{r}$ & 26 & 47 \\
\hline Patient 26 & 47 & $\mathrm{w}$ & 6 & 01.06 .1985 & $\mathrm{r}$ & 6 & \\
\hline Patient 27 & 74 & $\mathrm{~m}$ & 15 & 01.06 .1993 & $\mathrm{r}$ & 20 & 32 \\
\hline Patient 28 & 70 & $\mathrm{~m}$ & 3 & 01.04 .1997 & $\mathrm{r}$ & 11 & 40 \\
\hline Patient 29 & 68 & $\mathrm{~m}$ & 21 & 01.05 .1996 & 1 & 2 & 9 \\
\hline Patient 30 & 52 & $\mathrm{w}$ & 11 & 15.01 .1997 & 1 & 13 & 23 \\
\hline Patient 31 & 22 & $\mathrm{w}$ & 13 & 05.01 .1997 & $\mathrm{r}$ & 8 & 30 \\
\hline Patient 32 & 37 & $\mathrm{~m}$ & 3 & 01.02 .1997 & 1 & 24 & 47 \\
\hline Patient 33 & 74 & $\mathrm{~m}$ & 12 & 24.04 .1997 & $\mathrm{r}$ & 12 & 27 \\
\hline Patient 34 & 22 & $\mathrm{w}$ & 11 & 01.02 .1996 & $\mathrm{r}$ & 23 & 35 \\
\hline
\end{tabular}


8.10 Anhang J: Werte der unspezifischen Vorhersage

\begin{tabular}{|c|c|c|c|c|c|c|c|}
\hline $\begin{array}{l}\text { Versuchsperso } \\
\text { n }\end{array}$ & Alter & Geschlecht & BDI & $\begin{array}{c}\text { Schädigungs- } \\
\text { zeitpunkt }\end{array}$ & Seite & $\begin{array}{c}\text { Momentaner } \\
\text { Stand } \\
\%\end{array}$ & $\begin{array}{c}\text { Vorhergesagter } \\
\text { Stand } \\
\%\end{array}$ \\
\hline Patient 1 & 36 & $\mathrm{~m}$ & 6 & 01.08 .1996 & $\mathrm{r}$ & & \\
\hline Patient 2 & 35 & $\mathrm{w}$ & 5 & 29.11 .1996 & $\mathrm{r}$ & & \\
\hline Patient 3 & 28 & $\mathrm{~m}$ & 0 & 11.08 .1996 & 1 & & \\
\hline Patient 4 & 68 & w & 4 & 02.03 .1997 & 1 & 60 & 80 \\
\hline Patient 5 & 51 & $\mathrm{~m}$ & 7 & 01.11 .1995 & $\mathrm{r}$ & 70 & 80 \\
\hline Patient 6 & 74 & w & 20 & 01.02 .1993 & $\mathrm{r}$ & 50 & \\
\hline Patient 7 & 54 & W & 16 & 06.12 .1996 & $\mathrm{r}$ & 60 & 80 \\
\hline Patient 8 & 47 & $\mathrm{~m}$ & 1 & 01.01 .1997 & 1 & & \\
\hline Patient 9 & 29 & $\mathrm{~m}$ & 9 & 31.10 .1996 & 1 & & \\
\hline Patient 10 & 67 & w & 6 & 01.01 .1995 & 1 & 35 & 50 \\
\hline Patient 11 & 66 & $\mathrm{~m}$ & 19 & 16.11.1989 & 1 & 35 & 35 \\
\hline Patient 12 & 61 & $\mathrm{~m}$ & 10 & 01.03 .1995 & $\mathrm{r}$ & 50 & 60 \\
\hline Patient 13 & 77 & w & 7 & 01.12 .1996 & $\mathrm{r}$ & 20 & 70 \\
\hline Patient 14 & 52 & $\mathrm{~m}$ & 12 & 10.09 .1996 & 1 & 40 & 60 \\
\hline Patient 15 & 62 & w & 3 & 08.01 .1997 & 1 & 50 & 75 \\
\hline Patient 16 & 70 & $\mathrm{~m}$ & 11 & 15.01.1997 & $\mathrm{r}$ & 62 & 90 \\
\hline Patient 17 & 84 & $\mathrm{~m}$ & 11 & 09.08 .1996 & $\mathrm{r}$ & 30 & 60 \\
\hline Patient 18 & 58 & w & 7 & 18.11 .1996 & $\mathrm{r}$ & 50 & 70 \\
\hline Patient 19 & 61 & $\mathrm{~m}$ & 12 & 01.05 .1996 & 1 & 55 & 57 \\
\hline Patient 20 & 65 & w & 7 & 01.06 .1994 & $\mathrm{r}$ & 70 & 100 \\
\hline Patient 21 & 68 & $\mathrm{~m}$ & 5 & 12.01.1997 & 1 & 50 & 90 \\
\hline Patient 22 & 45 & W & 16 & 01.11 .1996 & 1 & 60 & 90 \\
\hline Patient 23 & 69 & $\mathrm{~m}$ & 12 & 01.07 .1996 & 1 & 40 & 60 \\
\hline Patient 24 & 59 & $\mathrm{~m}$ & 15 & 27.10 .1996 & $\mathrm{r}$ & 50 & 50 \\
\hline Patient 25 & 54 & $\mathrm{~m}$ & 22 & 06.04 .1996 & $\mathrm{r}$ & 50 & 60 \\
\hline Patient 26 & 47 & W & 6 & 01.06 .1985 & $\mathrm{r}$ & 50 & 70 \\
\hline Patient 27 & 74 & $\mathrm{~m}$ & 15 & 01.06 .1993 & $\mathrm{r}$ & 25 & 35 \\
\hline Patient 28 & 70 & $\mathrm{~m}$ & 3 & 01.04.1997 & $\mathrm{r}$ & 70 & 80 \\
\hline Patient 29 & 68 & $\mathrm{~m}$ & 21 & 01.05 .1996 & 1 & 30 & 60 \\
\hline Patient 30 & 52 & $\mathrm{w}$ & 11 & 15.01.1997 & 1 & 15 & 50 \\
\hline Patient 31 & 22 & $\mathrm{w}$ & 13 & 05.01 .1997 & $\mathrm{r}$ & 70 & 90 \\
\hline Patient 32 & 37 & $\mathrm{~m}$ & 3 & 01.02.1997 & 1 & 75 & 75 \\
\hline Patient 33 & 74 & $\mathrm{~m}$ & 12 & 24.04 .1997 & $\mathrm{r}$ & 70 & 90 \\
\hline Patient 34 & 22 & $\mathrm{w}$ & 11 & 01.02 .1996 & $\mathrm{r}$ & 90 & 100 \\
\hline
\end{tabular}




\subsection{Anhang K: Ausmaß der Schädigung}

\begin{tabular}{|c|c|c|c|c|c|c|}
\hline Versuchsperson & Alter & Geschlecht & BDI & $\begin{array}{c}\text { Schädigungs- } \\
\text { zeitpunkt }\end{array}$ & Seite & $\begin{array}{c}\text { Ausmaß der Schädigung } \\
\text { (nach Boné, Ladurner \& } \\
\text { Pichler, 1988) }\end{array}$ \\
\hline Patient 1 & 36 & $\mathrm{~m}$ & 6 & 01.08 .1996 & $\mathrm{r}$ & 1 \\
\hline Patient 2 & 35 & $\mathrm{w}$ & 5 & 29.11 .1996 & $\mathrm{r}$ & 2 \\
\hline Patient 3 & 28 & $\mathrm{~m}$ & 0 & 11.08 .1996 & 1 & 2 \\
\hline Patient 4 & 68 & $\mathrm{w}$ & 4 & 02.03 .1997 & 1 & 2 \\
\hline Patient 5 & 51 & $\mathrm{~m}$ & 7 & 01.11 .1995 & $\mathrm{r}$ & 2 \\
\hline Patient 6 & 74 & $\mathrm{w}$ & 20 & 01.02 .1993 & $\mathrm{r}$ & 2 \\
\hline Patient 7 & 54 & $\mathrm{w}$ & 16 & 06.12 .1996 & $\mathrm{r}$ & 1 \\
\hline Patient 8 & 47 & $\mathrm{~m}$ & 1 & 01.01 .1997 & 1 & 2 \\
\hline Patient 9 & 29 & $\mathrm{~m}$ & 9 & 31.10 .1996 & 1 & 1 \\
\hline Patient 10 & 67 & $\mathrm{w}$ & 6 & 01.01 .1995 & 1 & 2 \\
\hline Patient 11 & 66 & $\mathrm{~m}$ & 19 & 16.11 .1989 & 1 & 1 \\
\hline Patient 12 & 61 & $\mathrm{~m}$ & 10 & 01.03 .1995 & $\mathrm{r}$ & 1 \\
\hline Patient 13 & 77 & $\mathrm{w}$ & 7 & 01.12 .1996 & $\mathrm{r}$ & 0 \\
\hline Patient 14 & 52 & $\mathrm{~m}$ & 12 & 10.09 .1996 & 1 & 1 \\
\hline Patient 15 & 62 & $\mathrm{w}$ & 3 & 08.01 .1997 & 1 & 0 \\
\hline Patient 16 & 70 & $\mathrm{~m}$ & 11 & 15.01 .1997 & $\mathrm{r}$ & 1 \\
\hline Patient 17 & 84 & $\mathrm{~m}$ & 11 & 09.08 .1996 & $\mathrm{r}$ & 0 \\
\hline Patient 18 & 58 & $\mathrm{w}$ & 7 & 18.11 .1996 & $\mathrm{r}$ & 0 \\
\hline Patient 19 & 61 & $\mathrm{~m}$ & 12 & 01.05 .1996 & 1 & 1 \\
\hline Patient 20 & 65 & $\mathrm{w}$ & 7 & 01.06 .1994 & $\mathrm{r}$ & 1 \\
\hline Patient 21 & 68 & $\mathrm{~m}$ & 5 & 12.01 .1997 & 1 & 0 \\
\hline Patient 22 & 45 & $\mathrm{w}$ & 16 & 01.11 .1996 & 1 & 1 \\
\hline Patient 23 & 69 & $\mathrm{~m}$ & 12 & 01.07 .1996 & 1 & 2 \\
\hline Patient 24 & 59 & $\mathrm{~m}$ & 15 & 27.10 .1996 & $\mathrm{r}$ & 1 \\
\hline Patient 25 & 54 & $\mathrm{~m}$ & 22 & 06.04 .1996 & $\mathrm{r}$ & 1 \\
\hline Patient 26 & 47 & $\mathrm{w}$ & 6 & 01.06 .1985 & $\mathrm{r}$ & 2 \\
\hline Patient 27 & 74 & $\mathrm{~m}$ & 15 & 01.06 .1993 & $\mathrm{r}$ & 1 \\
\hline Patient 28 & 70 & $\mathrm{~m}$ & 3 & 01.04 .1997 & $\mathrm{r}$ & 1 \\
\hline Patient 29 & 68 & $\mathrm{~m}$ & 21 & 01.05 .1996 & 1 & 2 \\
\hline Patient 30 & 52 & $\mathrm{w}$ & 11 & 15.01 .1997 & 1 & 2 \\
\hline Patient 31 & 22 & $\mathrm{w}$ & 13 & 05.01 .1997 & $\mathrm{r}$ & 0 \\
\hline Patient 32 & 37 & $\mathrm{~m}$ & 3 & 01.02 .1997 & 1 & 1 \\
\hline Patient 33 & 74 & $\mathrm{~m}$ & 12 & 24.04 .1997 & $\mathrm{r}$ & 1 \\
\hline Patient 34 & 22 & $\mathrm{w}$ & 11 & 01.02 .1996 & $\mathrm{r}$ & 0 \\
\hline
\end{tabular}




\section{Danksagung}

Allen, die mich bei der Erstellung dieser Arbeit unterstützt haben, möchte ich herzlich danken. Mein besonderer Dank gilt:

- Herrn Prof. Dr. A. Krüger für die Vergabe des Themas sowie für die Betreuung und Förderung dieser Arbeit.

- Herrn Prof. Dr. A. Niklas für seine Bereitschaft, sich als Zweitgutachter zur Verfügung zu stellen.

- Frau C. Kraft und Herrn Dr. N. Schleicher, die durch ihre hilfreiche Unterstützung die Durchführung der Versuche in der Rehabilitationseinrichtung 'rehamed-Neuro' ermöglicht haben.

- Frau A. Kirn und Frau D. Schindler für ihre engagierte und zuverlässige Art, in der sie die Untersuchungen durchgeführt haben.

- Frau P. Krause, Frau J. Schwartz-Uppendieck und Herrn J. Krasel für ihr Engagement und ihre Geduld bei der Durchsicht dieser Arbeit.

- Meinen Eltern, die mir durch ihre bedingungslose Unterstützung so viel ermöglicht haben. 
Ich versichere, daß ich die eingereichte Dissertation „Sadder but wiser - Realismus der Selbsteinschätzung hinsichtlich der Belastungswahrnehmung und der motorischen Funktionswiederherstellung bei ZNS-Schädigungen in Abhängigkeit vom Grad der Depressivität“ selbständig und ohne unerlaubte Hilfsmittel verfaßt habe. Anderer als der von mir angegebenen Hilfsmittel und Schriften habe ich mich nicht bedient. Alle wörtlich oder sinngemäß den Schriften anderer Autoren entnommenen Stellen habe ich kenntlich gemacht.

Aichwald, den 28. Oktober 1997

Unterschrift:

(Stefan Krause) 